Florida International University FIU Digital Commons

\title{
Stochastic Geometry Based Analysis of Capacity, Mobility and Energy Efficiency for Dense Heterogeneous Networks
}

Arvind Merwaday

Florida International University, amerw001@fiu.edu

DOI: $10.25148 /$ etd.FIDC000245

Follow this and additional works at: https://digitalcommons.fiu.edu/etd

Part of the Systems and Communications Commons

\section{Recommended Citation}

Merwaday, Arvind, "Stochastic Geometry Based Analysis of Capacity, Mobility and Energy Efficiency for Dense Heterogeneous Networks" (2016). FIU Electronic Theses and Dissertations. 2480.

https://digitalcommons.fiu.edu/etd/2480 


\section{FLORIDA INTERNATIONAL UNIVERSITY}

Miami, Florida

\section{STOCHASTIC GEOMETRY BASED ANALYSIS OF CAPACITY, MOBILITY AND ENERGY EFFICIENCY FOR DENSE HETEROGENEOUS NETWORKS}

A dissertation submitted in partial fulfillment of the requirements of the degree of DOCTOR OF PHILOSOPHY in

ELECTRICAL ENGINEERING

by

Arvind Merwaday 
To: Interim Dean Ranu Jung

College of Engineering and Computing

This dissertation, written by Arvind Merwaday, and entitled Stochastic Geometry Based Analysis of Capacity, Mobility and Energy Efficiency for Dense Heterogeneous Networks, having been approved in respect to style and intellectual content, is referred to you for judgment.

We have read this dissertation and recommend that it be approved.

\begin{tabular}{r} 
Jean H. Andrian \\
\hline Armando Barreto \\
\hline Niki Pissinou \\
\hline Sayandev Mukherjee \\
İsmail Güvenç, Major Professor
\end{tabular}

Date of Defense: March 29, 2016

The dissertation of Arvind Merwaday is approved.

Interim Dean Ranu Jung College of Engineering and Computing

Andrés G. Gil

Vice President for Research and Economic Development and Dean of the University Graduate School

Florida International University, 2016 


\section{DEDICATION}

I dedicate this dissertation to my parents, Maruthi Sa and Rajeshwari, who have been the supporting pillars for all my work. My sister Deepa and my wife Shruthi have never left my side and are very special. Without their love, patience, and understanding, the completion of this work would not have been possible.

I also dedicate this dissertation to my friends who have been a constant source of support and encouragement during the challenges of my student life. 


\section{ACKNOWLEDGMENTS}

I wish to thank and pay my gratitude to the members of my committee for their insightful comments, encouragement, and generous support. I am extremely thankful and express my deepest gratitude to my major professor, Dr. İsmail Güvenç, for his valuable guidance and immense support for completing this research. He steered me in the right direction during my research and has always been there for help whenever I ran into troubles. His guidance helped me in all the time of research and writing of this dissertation. Finally, I would like to thank my fellow lab mates for the stimulating discussions, and for all the fun we have had in the last four years.

This research was supported in part by the U.S. National Science Foundation under the Grant CNS-1406968: NeTS: JUNO: "Energy-Efficient Hyper-Dense Wireless Networks with Trillions of Devices". 


\title{
ABSTRACT OF THE DISSERTATION \\ STOCHASTIC GEOMETRY BASED ANALYSIS OF CAPACITY, MOBILITY AND \\ ENERGY EFFICIENCY FOR DENSE HETEROGENEOUS NETWORKS
}

by

\author{
Arvind Merwaday \\ Florida International University, 2016 \\ Miami, Florida \\ Professor İsmail Güvenç, Major Professor
}

In recent years, the increase in the population of mobile users and the advances in computational capabilities of mobile devices have led to an exponentially increasing traffic load on the wireless networks. This trend is foreseen to continue in the future due to the emerging applications such as cellular Internet of things (IoT) and machine type communications (MTC). Since the spectrum resources are limited, the only promising way to keep pace with the future demand is through aggressive spatial reuse of the available spectrum which can be realized in the networks through dense deployment of small cells. There are many challenges associated with such densely deployed heterogeneous networks (HetNets). The main challenges which are considered in this research work are capacity enhancement, velocity estimation of mobile users, and energy efficiency enhancement.

We consider different approaches for capacity enhancement of the network. In the first approach, using stochastic geometry we theoretically analyze time domain inter-cell interference coordination techniques in a two-tier HetNet and optimize the parameters to maximize the capacity of the network. In the second approach, we consider optimization of the locations of aerial bases stations carried by the unmanned aerial vehicles (UAVs) to enhance the capacity of the network for public safety and emergency communications, in case of damaged network infrastructure. In the third approach, we introduce a subsidization scheme for the service providers through which the network capacity can be improved by using regulatory power of the government. Finally, we consider the approach of device-todevice communications and multi-hop transmissions for enhancing the capacity of a network. 
Velocity estimation of high speed mobile users is important for effective mobility management in densely deployed small cell networks. In this research, we introduce two novel methods for the velocity estimation of mobile users: handover-count based velocity estimation, and sojourn time based velocity estimation. Using the tools from stochastic geometry and estimation theory, we theoretically analyze the accuracy of the two velocity estimation methods through Cramer-Rao lower bounds (CRLBs).

With the dense deployment of small cells, energy efficiency becomes crucial for the sustained operation of wireless networks. In this research, we jointly study the energy efficiency and the spectral efficiency in a two-tier HetNet. We optimize the parameters of inter-cell interference coordination technique and study the trade-offs between the energy efficiency and spectral efficiency of the HetNet. 


\section{TABLE OF CONTENTS}

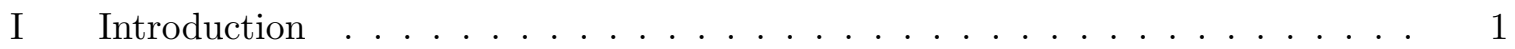

1.1 Capacity Enhancement . . . . . . . . . . . . . . . . . 2

1.2 Mobility Management . . . . . . . . . . . . . . . 8

1.3 Energy Efficiency Enhancement . . . . . . . . . . . . . . . . . . . 9

1.4 Contributions and Organization . . . . . . . . . . . . . . 10

II Literature Review . . . . . . . . . . . . . . . . . . . . . 13

2.1 Analysis of Capacity in HetNets using Stochastic Geometry . . . . . . . 13

2.2 UAVs in HetNets for Public Safety Communications . . . . . . . . . . . . 15

2.3 Spectrum Sharing and Subsidization for Capacity Enhancement . . . . . 17

2.4 Velocity Estimation and Mobility State Detection . . . . . . . . . . . 19

2.5 Energy Efficiency . . . . . . . . . . . . . . . . . . 21

III Analysis of FeICIC in two-tier HetNets . . . . . . . . . . . . . . . . . . . 23

3.1 System Model . . . . . . . . . . . . . . . . . . 23

3.2 Derivation of Joint SIR Distribution . . . . . . . . . . . . . . 27

3.3 Spectral Efficiency Analysis . . . . . . . . . . . . . . . . . 31

3.4 Numerical and Simulation Results . . . . . . . . . . . . . . . 35

IV Unmanned Aerial Base Stations in HetNets . . . . . . . . . . . . . . . . . 50

4.1 Public Safety Communications Scenarios . . . . . . . . . . . . . 50

4.2 System Model . . . . . . . . . . . . . . . . . . . . . . . 53

4.3 Coverage Improvement Using UABSs . . . . . . . . . . . . . . . . . 55

4.4 Simulation Results . . . . . . . . . . . . . . . . . . . 60

V Capacity Enhancement through Subsidy Regulations . . . . . . . . . . . . . 64

5.1 Introduction . . . . . . . . . . . . . . . . . 64

5.2 A Spectrum Market with Subsidy . . . . . . . . . . . . . 67

5.3 Perfect Nash Equilibrium . . . . . . . . . . . . . . . . . 75

5.4 A Simplified Spectrum Market . . . . . . . . . . . . . . . . . 78

5.5 Numerical Results . . . . . . . . . . . . . . . . . . . . . 84

VI Device-to-Device Communication and Multi-Hop Transmissions . . . . . . . . . 93

6.1 Channel Sounding Method with USRPs . . . . . . . . . . . . . . 94

6.2 Channel Propagation Modeling . . . . . . . . . . . . . . . . . 96

6.3 Measurement and Simulation Results . . . . . . . . . . . . . . . . . 99

VII Handover-Count based Velocity Estimation and Mobility State Detection . . . 104

7.1 System Model . . . . . . . . . . . . . . . 106

7.2 Approximation of the Handover Count PMF Using Gamma and

Gaussian Distributions . . . . . . . . . . . . . . . . . . . . 109

7.3 Cramer-Rao Lower Bound for Velocity Estimation . . . . . . . . . . . . 113

7.4 Mobility State Detection . . . . . . . . . . . . . . . 117 
7.5 Numerical Results . . . . . . . . . . . . . . . . . . . . . 121

VIII Sojourn Time based Velocity Estimation . . . . . . . . . . . . . . . . 133

8.1 System Model . . . . . . . . . . . . . . . . . . . . . . 133

8.2 CRLB for Velocity Estimation . . . . . . . . . . . . . . . 135

8.3 Sojourn Time Based Velocity Estimator . . . . . . . . . . . . . . . . 137

8.4 Numerical Results . . . . . . . . . . . . . . . . . . . . . . . 138

IX Energy Efficiency in HetNets . . . . . . . . . . . . . . . . . . . . 141

9.1 System Model . . . . . . . . . . . . . . . . . . . . . . 141

9.2 Numerical Results . . . . . . . . . . . . . . . . . . . . . . . . . 144

X Concluding Remarks and Future Work . . . . . . . . . . . . . . . 146

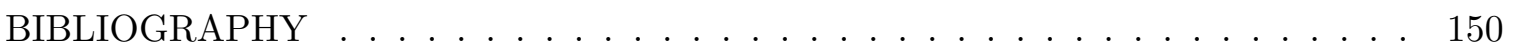

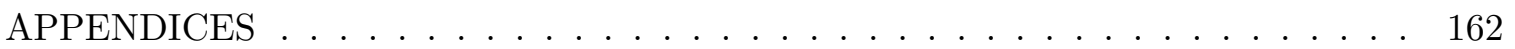

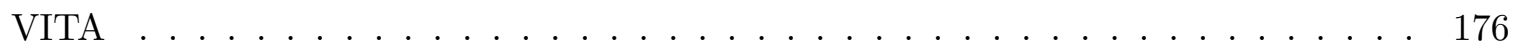




\section{LIST OF TABLES}

TABLE

PAGE

3.1. PPP parameters for USF MBSs, CSF MBSs, and PBSs. . . . . . . . . . . . 29

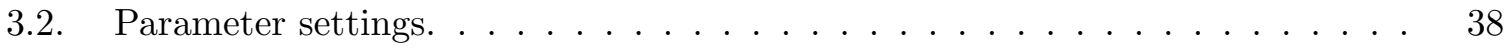

4.1. System parameters . . . . . . . . . . . . . . . . . 57

5.1. Notations and Symbols . . . . . . . . . . . . . . . . . . . . . 69

5.2. Statistics of the number of iterations for convergence. . . . . . . . . . 86

5.3. Details of the two regions and the two providers. . . . . . . . . . . 87

5.4. Simulation parameters for subsidization model. . . . . . . . . . . . . 89

6.1. Parameters of channel model . . . . . . . . . . . . . . . . . . . . 103 


\section{LIST OF FIGURES}

FIGURE

PAGE

1.1. Illustration of a HetNet. . . . . . . . . . . . . . . . . . . 1

1.2. The Cube: vision for wireless network evolution. . . . . . . . . . . . . 2

1.3. Illustration of the coverage areas of picocells with and without range expansion. 4

1.4. Illustration of range expansion and inter-cell interference in two-tier HetNets. 5

1.5. Illustration of multi-hop transmissions in device-to-device (D2D) communica-

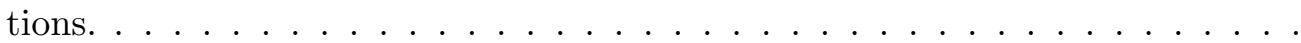

3.1. Frame structure with reduced power subframes, transmitted with a duty cycle

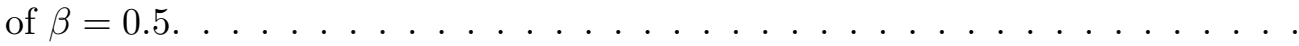

3.2. Illustration of user equipment (UE) association criteria. . . . . . . . . . . 26

3.3. Illustration of two-tier HetNet layout. . . . . . . . . . . . . . . . 27

3.4. Illustration of the integration region in the joint probability density function $(\mathrm{JPDF})$ of $X$ and $Y \ldots \ldots \ldots \ldots \ldots \ldots \ldots$

3.5. Illustration of the integration regions in the JPDF of $\Gamma$ and $\Gamma^{\prime} \ldots \ldots \ldots$

3.6. Simulation layout. . . . . . . . . . . . . . . . . . . 37

3.7. Per-user SE in macrocell and picocell with $\beta=0.5, \rho=4 \mathrm{~dB}$ and $\rho^{\prime}=0 \mathrm{~dB} . \quad 38$

3.8. Sum of $\log$ capacities versus the scheduling thresholds for different $\alpha$ and $\tau$

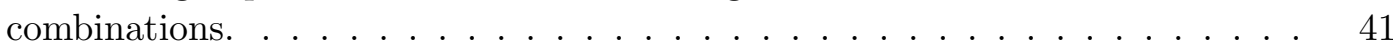

3.9. Optimized scheduling thresholds versus $\alpha$ for different $\tau$ (a) in macrocell; (b) in picocell. With $\lambda=4.6$ marks $/ \mathrm{Km}^{2}$ and $\lambda^{\prime}=13.8$ marks $/ \mathrm{Km}^{2} . \ldots .$.

3.10. $C_{\log }$ and $C_{\text {sum }}$ with optimum scheduling thresholds $\rho^{*}$ and $\rho^{\prime *} ; \lambda=4.6$ marks $/ \mathrm{Km}^{2}$ and $\lambda^{\prime}=13.8$ marks $/ \mathrm{Km}^{2} \ldots \ldots \ldots \ldots \ldots \ldots$. . . . . . . . . 44

3.11. Effects of $\alpha$ and $\tau$ on the 5th percentile, 50th percentile, and average SEs. (a) 5th percentile SE versus 50th percentile SE; (b) Average SE versus power reduction factor $\alpha$. With $\lambda=4.6$ marks $/ \mathrm{Km}^{2}$ and $\lambda^{\prime}=13.8$ marks $/ \mathrm{Km}^{2} \ldots$. .

3.12. $C_{\log }$ versus $\beta$ with optimum scheduling thresholds $\rho^{*}$ and $\rho^{*}$. With $\lambda=$ 4.6 marks $/ \mathrm{Km}^{2}$ and $\lambda^{\prime}=13.8$ marks $/ \mathrm{Km}^{2} \ldots \ldots \ldots \ldots$

3.13. $C_{\text {sum }}$ versus $\beta$ with optimum scheduling thresholds $\rho^{*}$ and $\rho^{*}$. With $\lambda=$ 4.6 marks $/ \mathrm{Km}^{2}$ and $\lambda^{\prime}=13.8$ marks $/ \mathrm{Km}^{2} \ldots \ldots \ldots \ldots . \ldots . \ldots 46$ 
3.14. 5th percentile throughput (a) in macrocell; (b) in picocell. With $\lambda=4.6$ marks $/ \mathrm{Km}^{2}$ and $\lambda^{\prime}=13.8$ marks $/ \mathrm{Km}^{2} \ldots \ldots \ldots \ldots \ldots \ldots$

3.15. Real base station locations of two different operators in a $15 \times 15 \mathrm{~km}^{2}$ area of London city. . . . . . . . . . . . . . . . . . . . .

3.16. 5th percentile SE versus picocell base station (PBS) density. . . . . . . . . .

4.1. Large scale PSC scenario. The MBSs, SCBSs, and UABSs constitute a heterogeneous network (HetNet) infrastructure, where the UABSs can dynamically change their positions for optimized coverage and seamless broadband connectivity. . . . . . . . . . . . . . . . . . . .

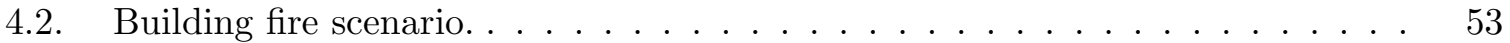

4.3. Illustration of SE coverage in a typical network. . . . . . . . . . . . . 55

4.4. An example of a chromosome, where the parameters that need to be optimized are the individual locations $\left(x_{i}, y_{i}\right)$ of each UABS $i$, where $i=1,2, \ldots, N \ldots$.

4.5. Illustration of a damaged network with optimized placements of UABSs using

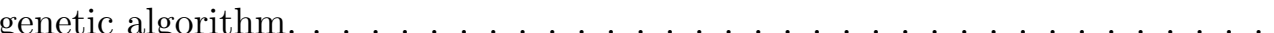

4.6. Improving SE coverage and 5 th percentile SE with UABSs. . . . . . . . . . 60

4.7. Effects of PLE on SE coverage area and 5 th percentile SE. . . . . . . . . . 61

4.8. Effects of REB on SE coverage probability and 5th percentile SE. . . . . . . .

5.1. Model for a subsidized spectrum market. In this model, government allocates subsidy $\xi_{p}$ to provider $p$, which in return can utilize a portion $s_{p r}$ of it to improve the service quality (which is captured by $\psi_{p r}$ ) in region $r$. Provider $p$ charges fee $f_{p}$ to each of its customers and as a result collects a revenue $R_{p r}$

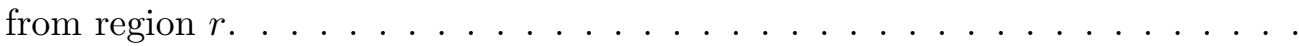

5.2. Flowchart of the iterative optimization method for finding optimized values of the subsidy amounts to be spent by the providers in different regions, and the optimum fee to be charged by the providers to the customers. . . . . . . . .

5.3. Illustration of penalty and reward functions. . . . . . . . . . . .

5.4. The convergence to equilibrium using best response algorithm. System parameters: $\xi_{1}=262, \xi_{2}=738, \beta=76, n_{1}=26, n_{2}=744, \gamma=0.05, \epsilon=10^{-3} . \ldots$.

5.5. Real BS locations of 2 providers in the cities of (a) Manchester, and (b) Leeds, in UK. . . . . . . . . . . . . . . . . . . . . 
5.6. Average per-user SE as a function of (a) number of BSs of provider $p$ in region $r$, and (b) subsidy amount spent by provider $p$ in region $r \ldots \ldots \ldots$

5.7. Optimum subsidy amounts spent by the providers and Optimum fee charged by the providers to each customer. . . . . . . . . . . . . . . .

5.8. Optimum objective functions of the providers and optimum social welfare. . . 91

5.9. Optimum allocation of the government's subsidy budget between the providers. 92

5.10. Social welfare and average per-user SE versus the government subsidy budget. 92

6.1. System setup for channel measurements. . . . . . . . . . . . . . . . . . 94

6.2. Aggregated spectrum of all the sinusoidal signals. . . . . . . . . . . . . . 95

6.3. Overlaid measurements of base-band equivalent (a) channel impulse responses; and (b) channel frequency responses; at a distance $d=21 \mathrm{~m}$ from the transmitter. 99

6.4. Average PDP of the channel for $d=21 \mathrm{~m} \ldots \ldots \ldots$. . . . . . . . . . . 99

6.5. Mean excess delay, RMS delay spread and coherence bandwidth of the channel. 100

6.6. Measured average path loss and the best line fit for different distances. . . . . 100

6.7. Fitting of Gaussian distribution to the histograms of the channel parameters

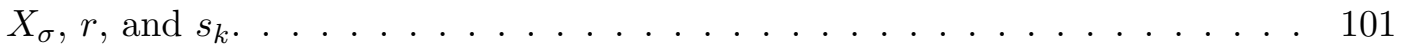

6.8. Comparison of the (a) measured, and (b) simulated PDP samples for different distances. . . . . . . . . . . . . . . . . . 102

6.9. Multi-hop communication. . . . . . . . . . . . . . . . . 102

6.10. BER for different distances and subcarrier frequency spacings. . . . . . . . . . 103

7.1. Handover failure problem in HetNets due to small cell size. . . . . . . . . . . . 105

7.2. Illustration of cellular network layout. . . . . . . . . . . . . . . 107

7.3. The PMF of the handover count for different $\lambda$ and $v$ values; (a) $\lambda=100 \mathrm{SBSs} / \mathrm{km}^{2}$; (b) $\lambda=200 \mathrm{SBSs} / \mathrm{km}^{2}$; (c) $\lambda=500 \mathrm{SBSs} / \mathrm{km}^{2}$; (d) $\lambda=1000 \mathrm{SBSs} / \mathrm{km}^{2} \ldots \ldots$

7.4. Illustration of fitting gamma distribution to the handover count PMF. . . . . 110

7.5. (a) PMF of the number of handovers; (b) PMF of the estimated velocity; $v=60 \mathrm{~km} / \mathrm{h}, \lambda=1000 \mathrm{SBSs} / \mathrm{km}^{2}, T=12 \mathrm{~s}, v_{\mathrm{l}}=40 \mathrm{~km} / \mathrm{h}$ and $v_{\mathrm{u}}=80 \mathrm{~km} / \mathrm{h} . \quad 121$

7.6. MSE versus $\lambda$ for different $v$, and $T=12 \mathrm{~s} . \ldots \ldots \ldots \ldots \ldots$ 
7.7. CRLB versus $v$ for different $\lambda$, and $T=12$ s. . . . . . . . . . . . 123

7.8. CRLB versus $T$ for different $\lambda$ and $v=60 \mathrm{~km} / \mathrm{h} . \ldots \ldots \ldots \ldots$. . . . . . 124

7.9. Comparison between the CRLB and the variance of the MVU estimator. . . . 125

7.10. Mobility state probabilities versus UE velocity; $T=12 \mathrm{~s}, v_{\mathrm{l}}=40 \mathrm{~km} / \mathrm{h}$ and

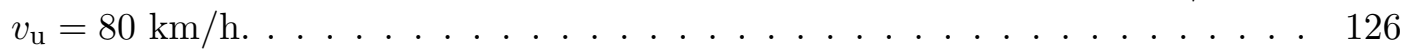

7.11. Probabilities of detection and false alarm. . . . . . . . . . . 126

7.12. Average probability of detection for different combinations of $h_{\mathrm{l}}$ and $h_{\mathrm{u}}$, with $\lambda=500 \mathrm{SBSs} / \mathrm{km}^{2} \ldots \ldots \ldots \ldots \ldots \ldots \ldots \ldots \ldots$

7.13. RMSE performance of the velocity estimator with RWP mobility model. . . . 128

7.14. Demonstration of velocity estimation and MSD with variable UE velocity. . . 129

7.15. RMSE of velocity estimation in homogeneous and clustered SBS deployments. 130

7.16. Realizations of Matern HCP of type II with fixed $\lambda_{\text {hc }}$ and different $\rho$ values;

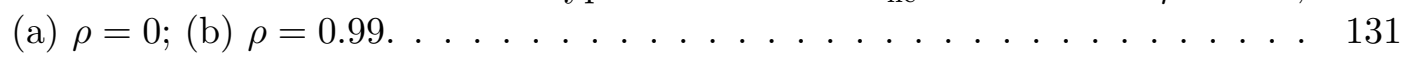

7.17. RMSE of velocity estimation with type II Matern HCP for the SBS locations. 132

8.1. Poisson-Voronoi tessellation of small cells and the UE travel trajectory. . . . . 134

8.2. Sojourn time PDF for different SBS densities, with $v=60 \mathrm{~km} / \mathrm{h} . \ldots \ldots$

8.3. CRLB and estimator variance versus $N$, for different UE velocities. . . . . 138

8.4. Comparison between the RMSEs of handover count and sojourn time methods for the velocity estimation of a UE. . . . . . . . . . . . . . 139

9.1. Frame structure at MBS and PBS with reduced power subframes. . . . . . . 142

9.2. Cell selection and scheduling criteria in different subframes. . . . . . . . . . 142

9.3. Illustration of two-tier HetNet layout. . . . . . . . . . . . . . . . 143

9.4. Optimum EE and corresponding $\alpha$, versus PBS density. . . . . . . . . . 144

9.5. Optimum $C_{\log }$ and corresponding $\alpha$, versus PBS density. . . . . . . . . 145

A3.1. Illustration for scaling down the simulation space. . . . . . . . . . . . 167

A3.2. Approximations of the $\alpha$ and $\beta$ parameters. . . . . . . . . . . 168

A4.1. Approximation of the $\sigma^{2}$ parameter. . . . . . . . . . . 169 


\section{ABBREVIATIONS AND ACRONYMS}

$\begin{array}{ll}\text { 3GPP } & \text { 3rd Generation Partnership Project } \\ \text { BER } & \text { bit error rate } \\ \text { BS } & \text { base station } \\ \text { CBRS } & \text { Citizen's Broadband Radio Service } \\ \text { CDF } & \text { cumulative distribution function } \\ \text { CIR } & \text { channel impulse response } \\ \text { CQI } & \text { channel quality indicator } \\ \text { CRLB } & \text { Cramer-Rao lower bound } \\ \text { CSF } & \text { coordinated subframe } \\ \text { D2D } & \text { device-to-device }\end{array}$

EARTH Energy Aware Radio and neTwork tecHnologies

EE energy efficiency

eICIC enhanced inter-cell interference coordination

EU European Union

FBS femtocell base station

FCC Federal Communications Commission

FeICIC further-enhanced inter-cell interference coordination

GA genetic algorithm

GPS global positioning system

HCPP hardcore point process

HDHN hyper-dense heterogeneous network

HetNet heterogeneous network

ICIC inter-cell interference coordination

IoT internet of things

JCCDF joint complimentary cumulative distribution function

JPDF joint probability density function

JRRM joint radio resource management 


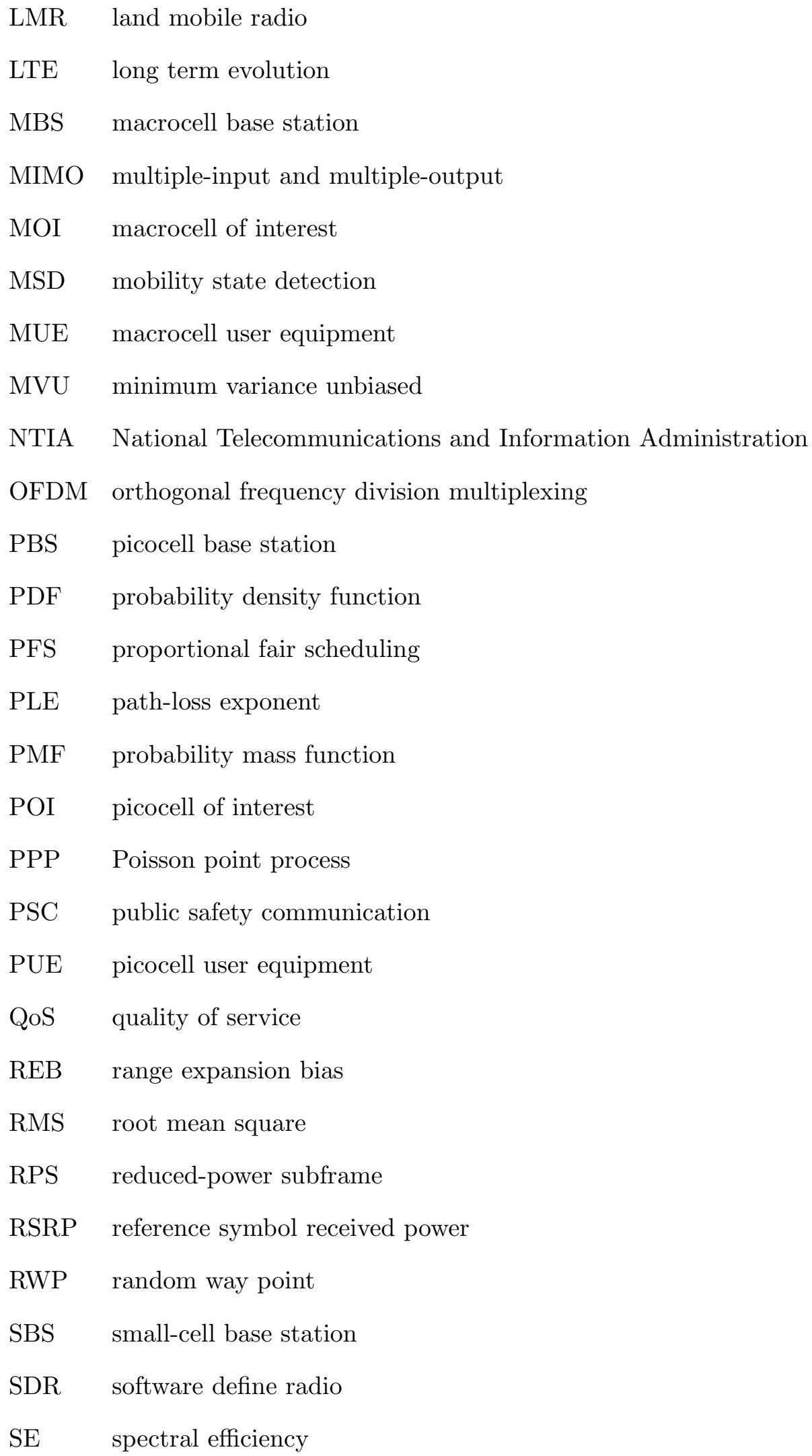




$\begin{array}{ll}\text { SINR } & \text { signal to interference plus noise ratio } \\ \text { SIR } & \text { signal to interference ratio } \\ \text { SON } & \text { self optimizing network } \\ \text { TTT } & \text { time-to-trigger } \\ \text { UABS } & \text { unmanned aerial base station } \\ \text { UAV } & \text { unmanned aerial vehicle } \\ \text { UE } & \text { user equipment } \\ \text { UK } & \text { United Kingdom } \\ \text { USF } & \text { uncoordinated subframe } \\ \text { USRP } & \text { universal software radio peripheral } \\ \text { WiFi } & \text { Wireless Fidelity }\end{array}$




\section{CHAPTER I}

\section{Introduction}

Since the introduction of advanced mobile devices with data-intensive applications, cellular networks are witnessing rapidly increasing data traffic demands from mobile users. Cisco has recently predicted an 11-fold increase in global mobile data traffic between 2013 and 2018 [1], while Qualcomm has predicted an astounding 1000x increase in mobile data traffic in the near future [2]. To keep up with the increasing traffic demands, the cellular networks are being transformed into heterogeneous networks (HetNets) by the deployments of small cells (picocell, femtocell, etc) over the existing macrocells. A HetNet is illustrated in Figure 1.1, which is composed of small-size cells such as micro-, pico-, and femto-cells overlaid on the existing macrocells to increase the frequency reuse and capacity of the network.

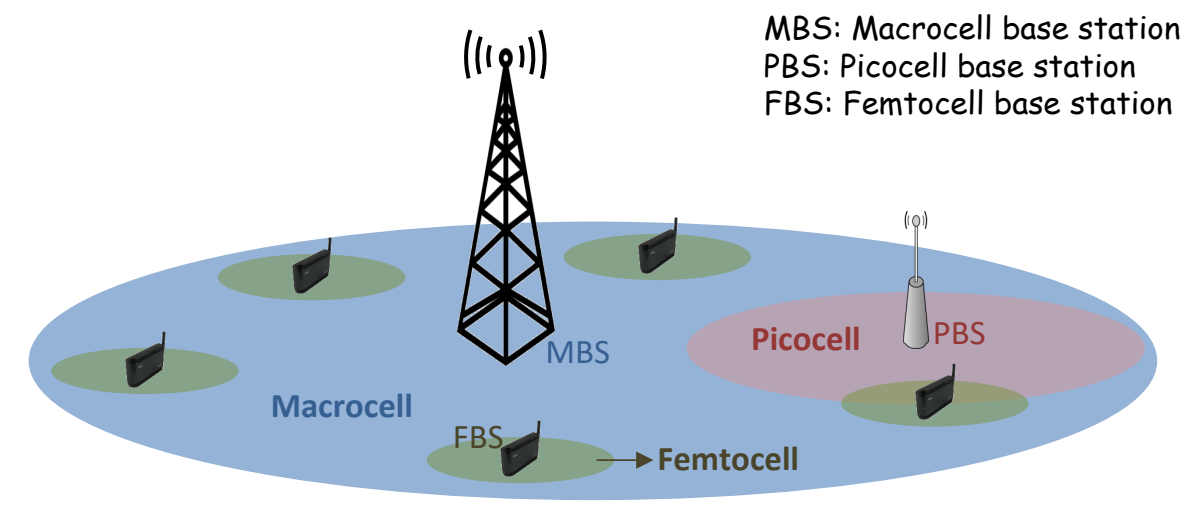

Figure 1.1: Illustration of a HetNet.

HetNets are a promising solution to achieve the ever increasing demand for higher data rates. However, it is inevitable for the network operators to face much bigger challenge in the future. Emerging applications such as massive internet of things (IoT) would multiply the number of devices on wireless communication networks by several folds, which would place tremendous traffic load on the network. Addressing this challenge will lead to extreme densification of the small cells which will give rise to hyper-dense heterogeneous networks (HDHNs). Although HDHNs can improve the network capacity by manifolds through the spatial reuse of available spectrum, this increase in the network capacity comes with an 


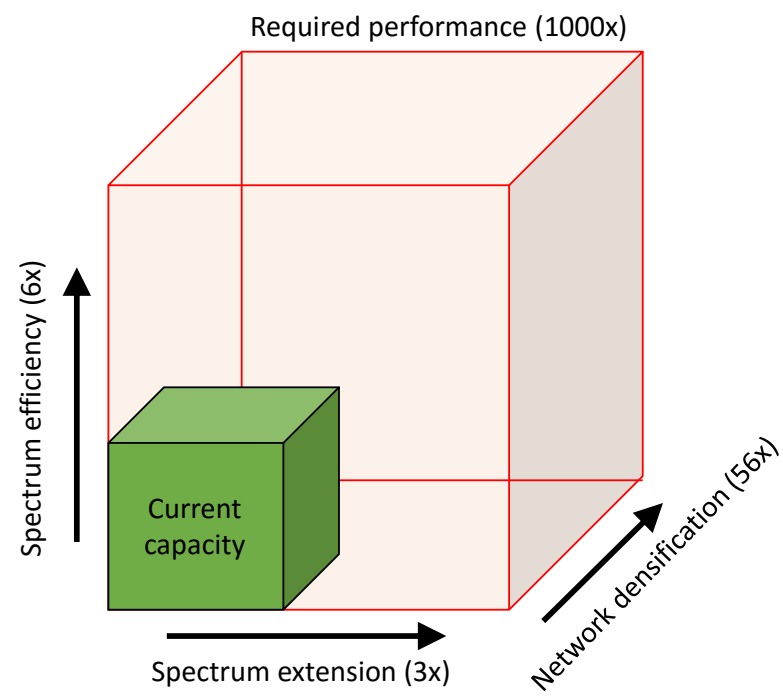

Figure 1.2: The Cube: vision for wireless network evolution.

increase in the interference and a need for complex interference coordination mechanisms. Hence, the extreme densification of small cells pose some critical challenges in the system design and management of the network. We classify these challenges into the following three broad categories: capacity enhancement, mobility management, and energy efficiency (EE) enhancement.

In summary, the daunting future data traffic demands can be met by substantially increasing the density of small cells. But, the extreme densification of small cells pose critical challenges in terms of mobility management and EE. Hence, to reap benefits from the next generation cellular networks, the three important parameters: capacity, mobility, and energy efficiency, should be analyzed and optimized by considering the associated tradeoffs. The goal of next generation cellular networks should be to provide an ubiquitous, efficient and greener mobile communication to the population.

\subsection{Capacity Enhancement}

Capacity enhancement is one of the main goals of the next generation wireless networks through which the exponentially increasing demand for wireless data can be met. The re- 
quired 1000x capacity improvement in the near future can be basically obtained through [3]: spectrum efficiency enhancement, spectrum extension, and network densification, as illustrated in Figure 1.2. It can be noticed that the majority (56x) of the required 1000x increase in network capacity is attributed to the network densification. Therefore, massive deployment of small cells in HetNets is imminent in the form of micro-, pico-, and femto-cells. A small portion $(6 \mathrm{x})$ of the capacity enhancement can be obtained through spectral efficiency (SE) enhancement techniques such as: coordinated multipoint transmissions and interference management techniques, multiple-input and multiple-output (MIMO) and beam forming techniques, etc. Since radio spectrum is a scarce and precious resource, only a minor portion $(3 \mathrm{x})$ of capacity enhancement can be expected from spectrum extension which relies on adding new licensed bands (including higher frequencies), and refarming of the existing bands for efficient use.

In this dissertation, the following innovative techniques for capacity enhancement in HetNets will be discussed and analyzed in detail.

1. Inter-cell interference coordination (ICIC) techniques to improve the channel conditions for the users experiencing severe interference from the neighboring cell base stations (BSs).

2. Fostering of spectrum sharing among the service providers through subsidization schemes from the government.

3. Device-to-device (D2D) communication and multihop transmissions for the efficient use of spectrum resources.

\subsubsection{Inter-cell Interference Coordination}

In HetNets, picocells and femtocells are primarily deployed in an attempt to improve the capacity of the cellular networks. The idea of small cell deployments is to reduce the traffic load on macrocells in crowded areas by offloading a portion of the macrocell users to the underlying small cells. However, due to smaller coverage areas, small cells might not be able to associate sufficient number of user equipments (UEs). To improve the UE 


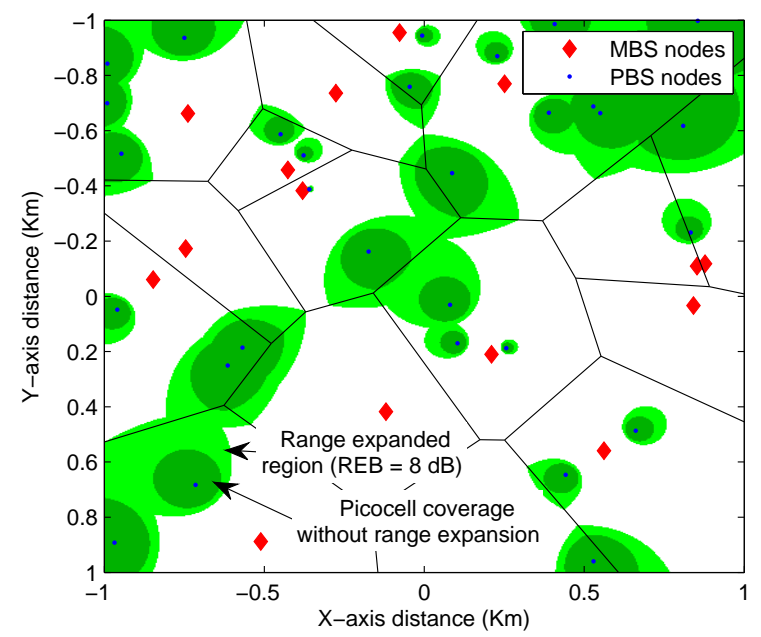

Figure 1.3: Illustration of the coverage areas of picocells with and without range expansion. offloading to small cells, range expansion bias (REB) is added to the reference symbol received powers (RSRPs) of the small cells during UE association, to virtually increase the coverage areas of small cells. For illustration, the coverage areas of the picocells with and without range expansion are shown in Figure 1.3. The use of REB enables a picocell to associate more users and thereby improving the offloading of UEs to the picocells. However, the picocell signal to interference plus noise ratio (SINR) in the range expanded region is lower than the macrocell SINR. As a result, the offloaded users in the range expanded region of picocells might experience high interference from the umbrella macrocell. This necessitates a ICIC mechanism between macrocell base stations (MBSs) and picocell base stations (PBSs) to protect the range expanded UEs from the MBS interference.

The ICIC techniques are inherently important in the context of HetNets. A HetNet basically consists of macrocells overlaid with small cells such as microcells, picocells, femtocells, etc. Consider a two-tier HetNet consisting of macrocells and cochannel deployment of picocells in which the picocells use same frequency band as the macrocells. In this scenario, the inter-cell interference is illustrated in Figure 1.4. For a picocell user equipment (PUE) located at the edge of the picocell, the received power from the neighboring MBS is comparable to the received power from the PBS. Therefore, the PUE will experience significant inter-cell interference from the MBS. The use of ICIC techniques are of critical importance in such a scenario. 


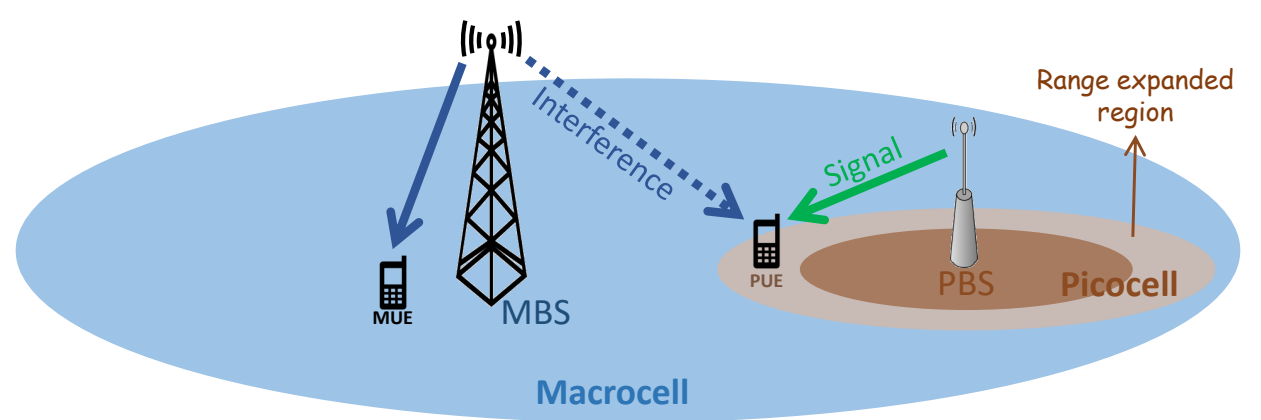

Figure 1.4: Illustration of range expansion and inter-cell interference in two-tier HetNets.

To mitigate the interference problems in HetNets, different enhanced inter-cell interference coordination (eICIC) techniques have been specified in long term evolution (LTE) Rel. 10 of 3GPP which includes time-domain, frequency domain and power control techniques [4]. In this dissertation, we mainly focus on the time-domain interference coordination methods. In the time domain eICIC technique, MBS transmissions are muted during certain subframes and no data is transmitted to macrocell user equipments (MUEs). The picocell-edge users are served by PBS during these subframes (coordinated subframes) and thereby protecting the picocell-edge users from MBS interference. In LTE Rel. 11, furtherenhanced inter-cell interference coordination (FeICIC) technique has been standardized in which the MBS transmits at reduced power (instead of muting the MBS completely) during the coordinated subframes (CSFs) to serve only its nearby UEs.

In this dissertation, the FeICIC technique will be theoretically studied on a system level using stochastic geometry. The key system parameters for FeICIC will be optimized by considering aggregate capacity in a cell and scheduling fairness among the users in a cell. Further, the potential benefits of unmanned aerial base station (UABS) cells will be studied in the post disaster scenario such as aftermath of an earthquake when a portion of the network infrastructure might have been damaged. Herein, the capacity and coverage gains that can be obtained by optimizing the locations of the UABSs (to minimize inter-cell interference) will be studied. 


\subsubsection{HetNets with Unmanned Aerial Base Stations}

In recent years, unmanned aerial vehicles (UAVs) and drones are being increasingly used in various applications including security and control operations, search and rescue missions, monitoring, crop management, communications, etc. One of the interesting applications of UAVs that is being currently researched is the use of UAV as a small cell BS which can be referred to as UABS. One of the main advantage of UABSs is that they can be deployed rapidly, which can be very useful in public safety communications (PSCs) during emergency events. UAVs are uniquely suited for such PSC scenarios due to their mobility and self organization capabilities, which are invaluable for quickly delivering broadband connectivity at times and locations where most needed, through an agile, low-cost, and ubiquitous communication infrastructure. On the other hand, interference management in such networks is more challenging due to the mobility of UABSs. Therefore, developing and analyzing ICIC techniques for the PSC networks with UABSs is an important task.

\subsubsection{Government Subsidization Scheme for Spectrum Sharing}

With the continuous increase in mobile traffic, users and mobile communication services are facing the problem of spectrum shortages. Governments around the world are trying to find ways in which more spectrum can be made available not only for mobile use, but also for other services that involve the use of wireless broadband technologies such as weather

forecast and surveillance [5]. Moreover, wireless networking technologies are becoming a more critical platform for disaster management and public safety applications, which mandates better communications and interoperability by effectively exploiting under-utilized spectrum resources following a disaster [6]. Conceptualizing the next generation cellular technology shows that improvement in the spectrum regulations will become a critical consideration to meet the future traffic demands [7].

One promising but relatively underexplored approach to foster more sharing of the spectrum is via the use of regulatory power. Here, the idea is to subsidize the spectrum to the providers with lower costs in return of proof-of-sharing. Thus, the providers will 
be offered discounted spectrum bands, potentially at different locations, but will be asked to cover users not subscribed to them so as to maintain their subsidy incentives from the government. In this dissertation, a spectrum sharing framework based on game theory will be introduced that considers spatial and temporal provider/government relationships, as well as dynamic components such as roaming and signal quality, in a noncooperative game. It will be shown that through spectrum subsidization, the SE of the users can be improved significantly while on the other hand, monopolization of the market by a single provider can be avoided.

\subsubsection{Device-to-Device Communication and Multihop Transmissions}

The D2D communication has recently received significant interest due to its potential ben-

efits to achieve higher throughput and offer proximity based services $[8,9]$. As a technology component of ongoing LTE Rel-12 standardization, it is also considered an important enabler for PSCs as it can be deployed rapidly with or without fixed infrastructure [10]. In general, the devices of a D2D network are battery powered and have stringent transmit power requirements. Therefore, the devices can benefit from multi-hop transmissions with the peer devices acting as relay nodes. In such a multi-hop network, the devices can communicate with one another over a much larger geographical area.

Consider a D2D scenario as illustrated in Figure 1.5 where the device D1 may communicate with D4 directly through single-hop transmission or through multi-hop transmission by using D2 and D3 as relay nodes. With transmit power control in the devices, the SINR at all the receivers are assumed to be constant. Then, the area of the large blue circle can be intuitively thought of as the power spent by D1 to communicate with D4. With this intuition, in the multi-hop case, the total area of the three small orange circles is smaller than the large blue circle. Thus, the total power spent in multi-hop case is smaller than that of the single-hop case. Moreover, larger coverage circle of D1 in single-hop case indicates higher interference caused to other devices. Therefore, multi-hop communication minimizes the interference while the devices can achieve longer distance communication. In 


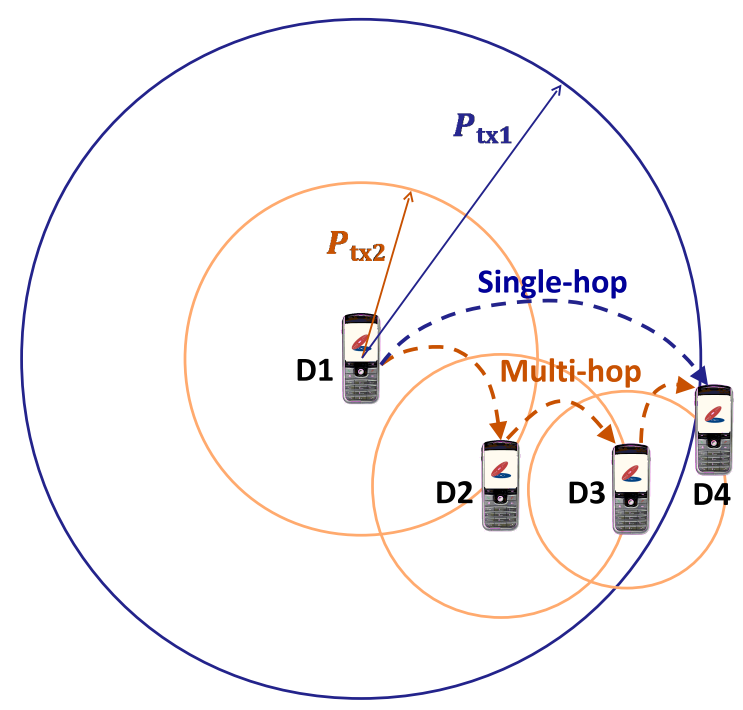

Figure 1.5: Illustration of multi-hop transmissions in D2D communications.

this dissertation, the advantage of multi-hop transmissions in terms of bit error rate (BER) performance will be studied by using link level simulations.

\subsection{Mobility Management}

The demand for wireless data traffic has increased significantly in the past decade and is expected to continue to do so $[1,2]$. Small cells are being rapidly deployed over existing macro-cellular networks in order to keep up with the increasing data traffic demands. Due to the random and dense deployment of small cells, mobility management has emerged as one of the most critical challenges, particularly when dealing with high-speed UE. Mobility management in cellular networks is an important task that is critical to provide good quality of service to the mobile users by minimizing the handover failures. Velocity of a UE plays critical role on the handover performance of the UE particularly when the cell density is high [11-13], where knowing the UE's velocity becomes necessary for effective mobility management. In homogeneous networks that only have macrocells, handovers are typically finalized at the cell edge due to large cell sizes. With the deployment of small-cell base stations (SBSs), due to smaller cell sizes, it becomes difficult to finalize the handover process at the cell edge for mobile devices $[11,12,14]$. In particular, high-mobility devices may run 
deep inside the coverage areas of small cells before finalizing a handover, thus incurring handover failure due to degraded SINR. These challenges motivate the need for UE-specific and cell-specific handover parameter optimization, which typically require estimation of the UE's velocity for effective configuration of handover parameters [15] such as: TTT, hysteresis threshold, etc. UE's velocity estimate may also be used for scheduling [16,17], mobility load balancing [18], channel quality indicator (CQI) feedback enhancements [19], and EE enhancements [20].

It is possible to develop efficient methods of velocity estimation by using the information generated from a mobile UE during the events of small cell boundary crossings. To this end, two novel and efficient velocity estimation methods are introduced and analyzed in this dissertation.

1. Handover-count based velocity estimation: In this method, the velocity of a UE is estimated based on the number of small cells traversed by the UE within a predefined time window. Here, the term "handover-count" is used in a broad sense to refer to the number of cells traversed by a UE, irrespective of whether or not the UE connects to the new cell after a small cell boundary crossing.

2. Sojourn time based velocity estimation: In this method, sojourn time samples of a mobile UE is used to estimate its velocity. The sojourn time is defined as the amount of time a mobile UE spends in a cell before it is handed over to another cell.

\subsection{Energy Efficiency Enhancement}

Alongside the increasing node density in HetNets, the remarkable growth of mobile communications industry has raised concerns over the energy consumption of the network infrastructure and the resulting atmospheric emissions. Indeed, mobile communications is expected to generate 201 Mega-tons of emissions by 2020. The telecom industry is concerned about the energy costs and carbon footprint of their network infrastructure. These concerns will get more critical in the future due to the increasing base station (BS) densities. In future generation HetNets, it is expected that the small cells can be as close as $50 \mathrm{~m}$ 
apart from each other [21]. In such a scenario, minimizing the network energy consumption while maximizing the data sent to the users becomes crucial for the sustained operation of the network.

The EE can be defined as

$$
E E=\frac{\text { Area SE }}{\text { Area power consumption }}
$$

where, "Area SE" is the achieved SE per unit area in bps $/ \mathrm{Hz} / \mathrm{km}^{2}$, and "Area power consumption" is the power consumption of the network infrastructure per unit area in $\mathrm{W} / \mathrm{km}^{2}$. According to (1.1), increasing the area SE will increase the EE. However, increasing the area SE might involve setting up of more BSs which will also increase the area power consumption. Therefore, there is a trade-off associated between the SE enhancement and the EE enhancement. In the HetNets, interference coordination techniques such as FeICIC plays an important role in improving the SE and EE of the network. In this research, we will analyze the SE and EE enhancements in a two-tier HetNet that uses FeICIC for interference coordination between the MBSs and PBSs. We will optimize the system parameters of FeICIC technique and study the trade-offs between SE and EE enhancements.

\subsection{Contributions and Organization}

The focus of this research is on 3 important requirements for the next generation HetNets: capacity enhancement, mobile velocity estimation, and EE enhancement. The research to be presented in this dissertation resulted in several publications in IEEE conferences and journals [22-33]. The key contributions of this dissertation can be summarized as follows.

\section{Capacity enhancement:}

- Generalized SE expressions are derived using stochastic geometry by considering the FeICIC method which includes eICIC and no eICIC as the two special cases. In this analytic framework that uses reduced power subframes and range expansion, expressions for the average SE of UEs and the 5th percentile throughput are 
derived. These expressions are validated through system level simulations. Further, optimization of key system parameters of the FeICIC method is performed in order to maximize the aggregate capacity in a cell and the proportional fairness among the users in the cell $[22,30,31]$.

- Enhancing the capacity and coverage of the cellular network by deploying the UABSs in a PSC scenario will be studied through system level simulations. Herein, it will be assumed that a portion of the network infrastructure will be damaged in a large geographical area due to a natural disaster such as an earthquake. In the simulations, the locations of the UABSs will be optimized using genetic algorithm $(G A)$ to maximize the 5 th percentile $S E$ of the users. Here, the benefits of using REB in the UABS cells will also be studied [25,28].

- A subsidization framework will be modeled using an extensive form game, where the government incentivizes the providers to give service to the foreign customers who are outside the coverage of their home provider. Through the numerical results, it will be shown that the government can significantly improve the average SE of the users by providing subsidy to the service providers. This study also shows that the government can avoid monopolization by a single provider and thereby offering a fair chance to the small service providers to compete in the market [26].

- The performance gains that can be obtained by using multi-hop transmissions in the D2D communications will be studied through link level simulations. Assuming that the devices use orthogonal frequency division multiplexing (OFDM) modulation scheme, the BER of two-hop transmissions will be shown to be considerably smaller than the single-hop transmissions [29].

\section{Mobile velocity estimation:}

- Handover-count based and sojourn time based velocity estimation methods will be introduced using the tools from stochastic geometry. The accuracy of the two estimation methods will be characterized through Cramer-Rao lower bounds 
(CRLBs). Additionally, the handover-count based velocity estimate will be used to detect the mobility state (low/medium/high) of a UE, and the expressions for the probability of detection and probability of false alarm will be derived. The CRLB of the sojourn time based method will be shown to be smaller than the CRLB of handover-count method $[23,24,27]$.

\section{Energy efficiency enhancement:}

- Using stochastic geometry, The EE and SE will be jointly studied by considering a two-tier HetNet which uses FeICIC and range expansion. The system parameters of FeICIC technique will be optimized and the trade-offs between EE enhancement and SE enhancement will be analyzed [33].

The remainder of this dissertation is organized as follows. Chapter II provides a literature review of the topics relevant to the issues discussed in the Introduction. The techniques of range expansion and FeICIC are analyzed theoretically in Chapter III for two-tier HetNets. The use of unmanned aerial BSs for capacity enhancement in HetNets is studied in Chapter IV. The framework for government subsidization is developed and analyzed in Chapter V. The advantages of using D2D communication and multi-hop transmissions in HetNets is studied through simulations in Chapter VI. In Chapters VII and VIII, handovercount based and sojourn time based techniques for the velocity estimation of a UE in dense HetNets are theoretically analyzed, respectively. Finally, the optimization of SE and EE in two-tier HetNets that use range expansion and FeICIC is studied in Chapter IX. Finally, Chapter $\mathrm{X}$ lists the conclusions of this research work and also identifies some important directions for future research. 


\section{CHAPTER II}

\section{Literature Review}

\subsection{Analysis of Capacity in HetNets using Stochastic Geometry}

Cellular networks are witnessing an exponentially increasing data traffic from mobile users. HetNets offer a promising way of meeting these demands. Since the BSs of different tiers in a HetNet use different transmission powers but the same spectrum bandwidth, analyzing and mitigating the interference at an arbitrary UE is difficult. Moreover, the locations of small cell BSs are random in nature as their deployments are not carefully planned. Even though the MBS locations are carefully planned, in reality, the actual deployments are random to some extent and do not resemble a standard pattern such as hexagonal grid. Therefore, modeling of the HetNets that are both realistic and mathematically tractable is a challenging task.

\subsubsection{Related Work on Evaluation Methodology}

Different approaches have been used in the literature for the performance evaluation of HetNets. The traditional simulation models with BSs placed on a hexagonal grid are highly idealized and may typically require complex and time-consuming system-level simulations. On the other hand, models based on stochastic geometry and spatial point processes provide a tractable and computationally efficient alternative for performance evaluation of HetNets [34-37]. Poisson point process (PPP) based models have been recently used extensively in the literature for performance evaluation of HetNets. However, as the MBS locations are carefully planned during the deployment process, PPP based models may not be viable for capturing real MBS locations, due to some points of the process being very close to each other. Matern hardcore point process (HCPP) provides a more accurate alternative spatial model for MBS locations. In HCPPs, the distance between any two points of the process is greater than a minimum distance predefined by hard core parameter. HCPP based models are relatively more complicated due to the non-existence of the probability 
generating functional [34]. Also, HCPP has a flaw of underestimating the intensity of the points that can coexist for a given hard core parameter [38]. Hence, HCPP models are not as tractable and simple as the PPP models.

With PPPs, using simplifying assumptions, such as Rayleigh fading channel model, and a path-loss exponent of four, we can obtain closed form expressions for aggregate interference and outage probability. Therefore, use of PPP models for performance evaluation of HetNets is appealing due to their simplicity and tractability [39]. Furthermore, the PPP based models provide reasonably close performance results when compared with the real BS deployments. In particular, results in [36] show that, when compared with real BS deployments, PPP and hexagonal grid based models for BS locations provide a lower bound and an upper bound, respectively, on the outage probabilities of UEs. Also, the PPP based models are expected to provide a better fit for analyzing denser HetNet deployments due to higher degree of randomness in small-cell deployments [35]. In this research, due to their simplicity and reasonable accuracy, we will use PPP based models to characterize and understand the behavior of HetNets in terms of various design parameters.

\subsubsection{Use of PPP Based Models for LTE-Advanced HetNet Performance Eval- uation}

The existing literature has numerous papers based on the PPP model for analyzing HetNets. Using PPPs, the basic performance indicators such as coverage probability and average rate of a UE are analyzed in [40-43]. The use of REB in the picocell enables it to associate with more UEs and thereby improves the offloading of UEs to the picocells. The effect of REB on the coverage probability is studied in [44,45]. However, with range expansion, the offloaded UEs at the edge of picocells experience high interference from the macrocell. This necessitates a coordination mechanism between the MBSs and PBSs to protect the picocell-edge UEs from the MBS interference. While $[35,36,46]$ considers a homogeneous cellular network, [45] considers a HetNet with range expansion. The authors of $[35,36,45]$ have obtained the information of real BS locations in an urban area from a cellular service provider. On the other hand, the authors of [46] have obtained the BS location information 
from an open source project [47] that provides approximate locations of the BSs around the world.

The eICIC technique using REB is studied well in the literature by analyzing its effects

on the rate coverage $[48,49]$ and on the average per-user capacity $[31,50]$. However, in the simulations of [51], the MBS transmits at reduced power (instead of muting the MBS completely) during the CSFs to serve only its nearby UEs. Therein, the use of reduced power subframes during CSFs is shown to improve the HetNet performance considerably in terms of the trade-off between the cell-edge and average throughputs. In another study [52], simulation results show that the FeICIC is less sensitive to the duty-cycle of CSFs than the eICIC. In [53], 3rd Generation Partnership Project (3GPP) compliant simulations are used to study and compare the eICIC and FeICIC techniques for different REBs and almost blank subframe densities. Therein, the amount of power reduction in the reduced power subframes is made equivalent to REB and its optimality is not justified. In [40], analytic expressions for coverage probability of an arbitrary UE is derived using PPPs. Later, the analytical framework in [40] has been extended to SE derivations in $[31,50]$ by considering eICIC and range expansion. Reduced power subframes, which are standardized in LTE Rel. 11 [54], are not analytically studied in literature to our best knowledge.

In this research, analytic expressions for SE in a network will be derived by including reduced power subframes and range expansion. The derived SE expressions will be validated through Monte Carlo simulations. Further, the key system parameters will be optimized to maximize both aggregate capacity in a cell and proportional fairness among its users.

\subsection{UAVs in HetNets for Public Safety Communications}

The PSCs carry critical importance to save lives, property, and national infrastructure in case of incidents such as fires, terrorist attacks, or natural disasters. Up until recently, PSC has been handled through narrowband communication technologies such as the land mobile radio (LMR), which can deliver reliable voice communications, but do not support broadband data [55], and are also often limited in terms of coverage and interoperability [56]. The National Broadband Plan by the Federal Communications Commission (FCC) states 
that a cutting-edge PSC shall make use of broadband technologies "to allow first responders anywhere in the nation to send and receive critical voice, video and data to save lives, reduce injuries and prevent acts of crime and terror".

Broadband wireless technologies such as the 4G LTE have a strong potential for revolutionizing communications during public safety situations. While the legacy LMR technology can provide better cell coverage and range when compared to LTE for PSC applications, LTE technology can offer substantially higher data rates, which are critical for services such as real-time situational awareness [57]. Exploiting the features of LTE systems will be essential for transforming the PSC infrastructures from a capacity-limited platform into a high-speed communication infrastructure. For example, efforts to develop the first nationwide, LTE-based high-speed PSC network, FirstNet [58], are in progress in the United States.

Another important opportunity for revolutionizing the PSC capabilities is to introduce UAVs, such as balloons, quadcopters, or gliders, for delivering pervasive broadband connectivity [59]. Enabled by recent technological advances, miniaturization, and open-source hardware/software initiatives, UAVs have found several key applications recently [60-63]. Amazon, for example, claims that seeing its Prime Air order delivery UAVs in the sky is expected to be as conventional as seeing mail trucks on the road within the next few years [62]. Google and Facebook have been investigating the use of a network of highaltitude balloons [64] and drones [65] over specific population centers for providing broadband connectivity. Such solar-powered drones are capable of flying several years without refueling. A relatively less explored application of UAVs is to deliver broadband data rates in emergency and public safety situations through low-altitude platforms [66].

In this research, we consider a large scale PSC scenario in which a portion of the network infrastructure is damaged due to a natural disaster. We investigate the potential benefits of UAVs in the post disaster scenario by studying the capacity and coverage improvements achieved through the deployment of UAVs. Furthermore, we optimize the locations of the UAVs to maximize the 5th percentile capacity of the network. 


\subsection{Spectrum Sharing and Subsidization for Capacity Enhancement}

In the United States, FCC and National Telecommunications and Information Administration (NTIA) are investing in the projects of spectrum sharing between Federal and non-Federal users as a means to increase spectrum capacity while increasing its efficiency as well [67]. For example, T-Mobile USA is currently sharing its spectrum with federal agencies [68]. The Citizen's Broadband Radio Service (CBRS) is another recent initiative in the United States [69] which aims to effectively share the federal spectrum with non-federal users under well defined FCC regulations. Cognitive radio and dynamic spectrum access techniques have been extensively studied in the literature [70-72], which can facilitate better sharing of the spectrum in these scenarios.

Subsidization of spectrum to the providers is a promising approach through which the government can use its regulatory power to encourage spectrum sharing among the providers. Recent studies suggest significant market and user welfare gains under subsidization schemes, e.g., data subsidy for offering minimal data plan to users for free [73] or spectrum pooling among providers to improve user experience [74]. To our best knowledge, study of such subsidized spectrum markets using game theoretical frameworks is not available in the literature. Even though subsidization is heavily employed in several markets, the wireless market has not seen much of its usage beyond a few, limited scenarios. The most

known subsidization in a wireless market is the subsidization of the expensive phones to the users by the provider [75], in that the user pays the phone's cost over a locked, termed contract. Unlocking the contract term either requires return of the phone or payment of a significant fee.

Only recently, the authors in [73] investigated the concept of using subsidies for spectrum management. Their work analyzed price and quality of service (QoS) subsidy schemes to increase the utility of the consumers from the data plans. The goal is to increase the availability of wireless data plans to more users. In their model, the regulator offers a subsidized (i.e., less expensive) data plan to the end users with lower quality of service via subsidization to the providers. In contrast, here, we consider subsidizing the spectrum to 
the providers and focus on implementing a subsidy system mainly between the providers and the government. We aim to make the subsidization seamless to the end users and aim to incentivize the providers to be more welcoming to the users subscribed to other providers. As a major difference, we focus on spectrum subsidy rather than data plan subsidy.

A related concept to the subsidization approach that we consider is inter-operator roaming. Through roaming, a customer can make/receive calls and send/receive data while outside of the home network coverage, by using the infrastructure of another provider. This is enabled through reciprocal roaming charges among operators, which may vary, e.g., depending on the nationwide coverage supported by an operator through its infrastructure. The new roaming regulations in European Union (EU) to be effective from June 2017 will no longer allow the telecom providers to charge extra roaming costs to their customers [76]. The goal is to facilitate competition in the roaming market and bring international roaming prices down to domestic rates, e.g., through diversity of market players, low barriers to market entry, and equal access to basic wholesale services.

The analysis in [77] shows that, as long as certain coverage conditions are fulfilled, providers in an open system (where roaming is allowed) have strictly better revenues when compared to a closed system with no roaming. Benefits of inter-operator spectrum sharing and joint radio resource management (JRRM) have also been demonstrated in recent works [78-80]. The inter-operator JRRM technique proposed in [78] allows subscribers to get service through other operators in case the home operator network is blocked, and results show that inter-operator roaming agreements improve both the network performance and the operators' revenue.

In this research, we model a subsidization framework using extensive form game, and show how to find (perfect) equilibrium solutions for (a simple case of) the game. The players in the game are a finite number of service providers plus a single government player. The government incentivizes by offering subsidy to the providers so that they give service to foreign customers who are outside the coverage of their home provider or simply in a spot where their home providers signal quality is worse than other providers they are not subscribed to. Yet, the government also expects the providers to share their spectrum 
and infrastructure resources with foreign calls. If a provider cannot satisfy a proof-ofsharing level, it has to return some or all of the subsidy back to the government. Thus, the providers each aim to maximize their profit from enrolling customers, by efficiently investing the subsidies received from the government.

\subsection{Velocity Estimation and Mobility State Detection}

Existing LTE and LTE-Advanced technologies are capable of estimating the mobility state of a UE into three broad classes: low, medium, and high-mobility $[11,12,15,81]$. This is achieved at a UE by counting the number of handovers within a given time window, and comparing it with a threshold which can be implemented during the connected mode [82] or the idle mode [83] of the UE. It can also be implemented at the network side, by tracking the prior history of handovers for a particular UE. The accuracy of a UE's mobility state estimate will benefit significantly from the densification of SBS deployments. While more accurate UE-side speed estimation techniques based on Doppler estimation have been discussed in [84-87], due to their complexity and standardization challenges, they have not been adopted in existing cellular network standards. While global positioning system (GPS) can be used for accurate estimation of a UE's speed, it may not be a practical solution for

mobility management as 1) the GPS receiver at a UE consumes significant amount of power, 2) GPS coverage may not be available in environments such as urban canyons and underground subways, and 3) not all the UEs are equipped with GPS receivers. A popular usecase example that uses such real-time mobility state information at a UE is that of UEspecific cell selection: high speed UEs can be biased to stay connected to macrocells even when small cell link quality is better [88].

\subsubsection{Handover-count Based Velocity Estimation}

There are various studies available in the literature on handover-count based mobility state detection (MSD). A simulation based mobility analysis is performed in [81] where it is proposed that the number of handovers made by a UE are weighted differently for macro to macro, macro to pico, pico to macro, and pico to pico handovers $(1,0.45,0.25$, and 0.1 , 
respectively), to produce a good estimate of UE mobility. In [89], considering a random way point (RWP) model and using stochastic geometry, the expected number of handovers during the movement period of a RWP model is derived. The probability density function (PDF) of the sojourn time is also presented for homogeneous networks. In [90], a theoretical framework using stochastic geometry is developed to study the UE mobility in HetNets, in which the expressions for vertical and horizontal handoff rates are derived. In [91], closed form expressions of cross-tier handover rate and sojourn time in a small cell are provided using stochastic geometry for a two-tier network. In another study [92], stochastic geometry is used to derive the handover rate, which in turn is used to derive coverage probability of a UE in HetNet by considering that the UE is mobile and a fraction of the handovers result into failure.

A set of related works in the literature have studied the path prediction of UEs and the handoff time estimation along the predicted path which can help in improving the quality of service to the UEs. A destination and mobility path prediction model called DAMP is proposed in [93], while a handoff time window estimation method and mobility-predictionaware bandwidth (MPBR) allocation scheme is presented in [94]. In [95], different methods for predicting the future location of a UE based on prior knowledge of the UE's mobility are studied. A method for tracing UE's location using semi-supervised graph Laplacian approach is proposed in [96].

Despite the earlier work in $[11,12,15,81-87,89,90,92-96]$, fundamental performance bounds and optimum algorithms for handover-count based velocity estimation have not been studied in the literature. In this research, we introduce a novel and efficient handovercount based UE velocity estimation technique using the tools from stochastic geometry, and characterize its accuracy through CRLBs, when the density of SBSs is known. We further extend our study to MSD of a UE. The focus of this research is on velocity estimation and MSD based on the handover counts of a UE; path prediction and handoff time estimation techniques are not considered. Mobility state estimation itself is an important topic in 3GPP Release- 8 specifications, and being researched currently. Additionally, there are applications which require velocity estimation at the UE side, in which case, implementing 
the path prediction algorithms at a UE would be difficult. To our best knowledge, velocity estimation based on handover counts at a UE has not been studied analytically in the literature, which is one of the main contribution of this part of the research.

\subsubsection{Sojourn Time Based Velocity Estimation}

There are only a few studies available in the literature that investigate the use of sojourn time information for mobility management. In [97], considering a RWP for UE mobility, expressions for the PDF and the expected value of the sojourn time are derived for homogeneous networks, assuming that the BSs are distributed according to a PPP. The authors in [98] estimated the velocity of a UE in two-tier cellular networks using the sojourn times of the UE, while assuming a classical Manhattan cell model [99]. Despite the earlier work in $[11,97,98]$, fundamental bounds for the accuracy of sojourn-time based UE velocity estimation have not been studied in the literature. In this research, we introduce a novel technique for UE velocity estimation based on sojourn-time samples, and analyze its accuracy through CRLBs, when the density of SBSs is known.

\subsection{Energy Efficiency}

Until recently, only SE was considered as the key performance indicator of cellular networks by the researchers and the $3 \mathrm{GPP}$ evolution. Nowadays, green evolution is becoming a major trend and the telecom industry is concerned about the energy costs and carbon footprint of their network infrastructure. The Energy Aware Radio and neTwork tecHnologies (EARTH) project [100] which was completed in 2012 has made several contributions in the literature. The goal of the EARTH project was to address the global environmental challenge by investigating the EE of mobile communication systems. The energy consumption of a MBS is far greater than the energy consumption of a small cell BS. Recent surveys reveal that around $80 \%$ of the energy required for the operation of a cellular network is consumed at the MBS sites [101]. Hence, deploying more MBSs to achieve better SE will lead to smaller EE when compared to the deployment of small cell BSs. In other words, by deploying more small cells the BSs can be brought closer to the users which results into 
smaller radiation of energy. Accordingly, the studies in [102-105] through Monte Carlo simulations show that the EE in HetNets is considerably better than the EE in homogeneous networks.

There are several other works in the literature which are focused on the EE of cellular networks. While the works in [106-109] are simulation oriented and consider hexagonal macrocell geometry or a single macrocell overlaid with small cells, the works in [110-116] are based on stochastic geometry. The current state of the art consists of several interesting techniques to improve the EE of future cellular networks. One promising approach is to put some BSs into sleep mode during certain times when the traffic load is low [106,108-111,115]. In [114], the authors have shown that phantom cell network (unlike small cell BSs, the phantom cell BSs operate on a different spectrum from the MBSs) has both higher SE and EE than the small cell network. Another technique is proposed in [112] where BSs from different tiers in a HetNet jointly transmit the same data to a typical user, and further optimize the EE performance. In another work [113], the authors have proposed cooperative and cognitive schemes to increase the EE in HetNets. To improve the EE in HetNets, an idea of DTX support for the BS resource scheduler is proposed in [107] such that the transmissions to a UE are delayed until there is a sufficient amount of data requiring transmission to utilize at least a defined percentage of the system bandwidth. However, to the best of our knowledge, there is no work in the literature that jointly studies the SE and EE in HetNets with FeICIC mechanism. In this research, we will study the optimization of FeICIC for EE and SE enhancements in a two-tier HetNet. 


\section{CHAPTER III \\ Analysis of FeICIC in two-tier HetNets}

The use of reduced power subframes has been standardized in LTE Rel. 11 which is therein referred to as FeICIC. The reduced power subframes in the FeICIC technique can improve the capacity of HetNets while also providing interference coordination to the picocell-edge users. However, in order to obtain maximum benefits from the reduced power subframes, setting the key system parameters, such as the amount of power reduction, carries critical importance. Using stochastic geometry, this chapter lays down a theoretical foundation for the performance evaluation of HetNets with reduced power subframes and range expansion bias. The analytic expressions for average capacity and 5th percentile throughput are derived as a function of transmit powers, node densities, and interference coordination parameters in a two-tier HetNet scenario, and are validated through Monte Carlo simulations. The details of the simulation model are documented explicitly, and the Matlab codes can be accessed through [117] for regenerating the results. Joint optimization of range expansion bias, power reduction factor, scheduling thresholds, and duty cycle of reduced power subframes are performed to study the trade-offs between aggregate capacity of a cell and fairness among the users. To validate our analysis, we also compare the stochastic geometry based theoretical results with the real MBS deployment (in the city of London) and the hexagonal-grid model. Our analysis shows that with optimum parameter settings, the LTE Rel. 11 with reduced power subframes (FeICIC) can provide substantially better

performance than the LTE Rel. 10 with almost blank subframes (eICIC), in terms of both aggregate capacity and fairness.

\subsection{System Model}

We consider a two-tier HetNet system with MBS, PBS and UE locations modeled as twodimensional homogeneous PPPs of intensities $\lambda, \lambda^{\prime}$ and $\lambda_{\mathrm{u}}$, respectively. Both the MBSs and the PBSs share a common transmission bandwidth. We assume round robin scheduling in all 
the downlinks of a cell. For analytical tractability, we also assume that during a subframe, a BS allocates entire system bandwidth to a single UE. We also assume that the cells have full buffer traffic and the thermal noise is negligible when compared to interference. The MBSs employ reduced power subframes, in which they transmit at reduced power levels to prevent high interference to the PUEs. On the other hand, the PBSs transmit at full power during all the subframes.

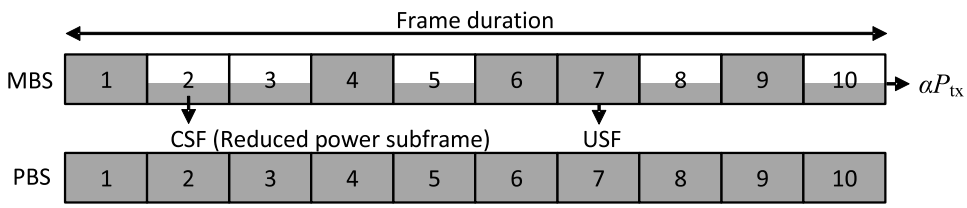

Figure 3.1: Frame structure with reduced power subframes, transmitted with a duty cycle of $\beta=0.5$.

The frame structure with reduced power subframes is shown in Figure 3.1. During uncoordinated subframes (USFs), the MBS transmits data and control signals at full power $P_{\mathrm{tx}}$ and during CSFs, it transmits at a reduced power $\alpha P_{\mathrm{tx}}$, where $0 \leq \alpha \leq 1$ is the power reduction factor. The PBS transmits the data, control signals and cell reference symbol with power $P_{\mathrm{tx}}^{\prime}$ during all the subframes. Setting $\alpha=0$ corresponds to eICIC; and $\alpha=1$ corresponds to no eICIC case.

Define $\beta$ as the duty cycle of USFs, i.e., ratio of the number of USFs to the total number of sub-frames in a frame. Then, $(1-\beta)$ is the duty cycle of CSF/reduced power subframes. Let $K$ and $K^{\prime}$ be the factors that account for geometrical parameters such as the transmitter and receiver antenna heights of the MBS and the PBS, respectively. Then, the effective transmitted powers of MBS during USFs is $P=P_{\mathrm{tx}} K$, MBS during CSFs is $\alpha P$, and PBS during USF/CSF is $P^{\prime}=P_{\mathrm{tx}}^{\prime} K^{\prime}$. For an arbitrary UE, let the nearest MBS at a distance $r$ be its macrocell of interest (MOI) and the nearest PBS at a distance $r^{\prime}$ be its picocell of interest (POI). Then, assuming Rayleigh fading channel, the reference symbol received power from the MOI and the POI are given by,

$$
S(r)=\frac{P H}{r^{\delta}}, \quad S^{\prime}\left(r^{\prime}\right)=\frac{P^{\prime} H^{\prime}}{\left(r^{\prime}\right)^{\delta}}
$$


respectively, where $\delta$ is the path-loss exponent, and the random variables $H \sim \operatorname{Exp}(1)$ and $H^{\prime} \sim \operatorname{Exp}(1)$ account for Rayleigh fading. Define an interference term, $Z$, as the total interference power at a UE during USFs from all the MBSs and the PBSs, excluding the MOI and the POI. Similarly, define $Z^{\prime}$ as the total interference power during CSFs. We assume that there is no frame synchronization across the MBSs and therefore irrespective of whether the MOI is transmitting a USF or a CSF, the interference at UE has the same distribution in both cases, and is independent of both $S(r)$ and $S^{\prime}\left(r^{\prime}\right)$. Then, an arbitrary UE experiences the following four signal to interference ratios (SIRs):

$$
\begin{aligned}
\Gamma & =\frac{S(r)}{S^{\prime}\left(r^{\prime}\right)+Z}, \rightarrow \text { USF SIR from MOI } \\
\Gamma^{\prime} & =\frac{S^{\prime}\left(r^{\prime}\right)}{S(r)+Z}, \rightarrow \text { USF SIR from POI } \\
\Gamma_{\mathrm{csf}} & =\frac{\alpha S(r)}{S^{\prime}\left(r^{\prime}\right)+Z}, \rightarrow \text { CSF SIR from MOI } \\
\Gamma_{\mathrm{csf}}^{\prime} & =\frac{S^{\prime}\left(r^{\prime}\right)}{\alpha S(r)+Z} \cdot \rightarrow \text { CSF SIR from POI }
\end{aligned}
$$

\subsubsection{UE Association}

In (3.4) and (3.5), it can be noted that $\Gamma_{\mathrm{csf}}$ and $\Gamma_{\mathrm{csf}}^{\prime}$ are directly affected by $\alpha$ and hence their usage will make the cell selection process dependent on $\alpha$. Thus, we consider $\Gamma$ and $\Gamma^{\prime}$ to minimize the dependence of the cell selection process on $\alpha$.

The cell selection process using $\Gamma, \Gamma^{\prime}$ and the $\operatorname{REB} \tau$ can be explained with reference to Figure 3.2. If $\tau \Gamma^{\prime}$ is less than $\Gamma$, then the UE is associated with the MOI, otherwise with the POI. After the cell selection, the UE is scheduled either in USF or in CSF based on the scheduling thresholds $\rho$ (for MUE) and $\rho^{\prime}$ (for PUE). In macrocell, if $\Gamma$ is less than $\rho$ then the UE is scheduled to USF, otherwise to CSF. Similarly, in picocell, if $\Gamma^{\prime}$ is greater than $\rho^{\prime}$ then the UE is scheduled to USF, otherwise to CSF (to protect it from macrocell interference). The cell selection and scheduling conditions can be combined and formulated 
as:

$$
\begin{aligned}
& \text { If } \Gamma>\tau \Gamma^{\prime} \text { and } \Gamma \leq \rho \rightarrow \text { USF-MUE, } \\
& \text { If } \Gamma>\tau \Gamma^{\prime} \text { and } \Gamma>\rho \rightarrow \text { CSF-MUE, } \\
& \text { If } \Gamma \leq \tau \Gamma^{\prime} \text { and } \Gamma^{\prime}>\rho^{\prime} \rightarrow \text { USF-PUE, } \\
& \text { If } \Gamma \leq \tau \Gamma^{\prime} \text { and } \Gamma^{\prime} \leq \rho^{\prime} \rightarrow \text { CSF-PUE. }
\end{aligned}
$$

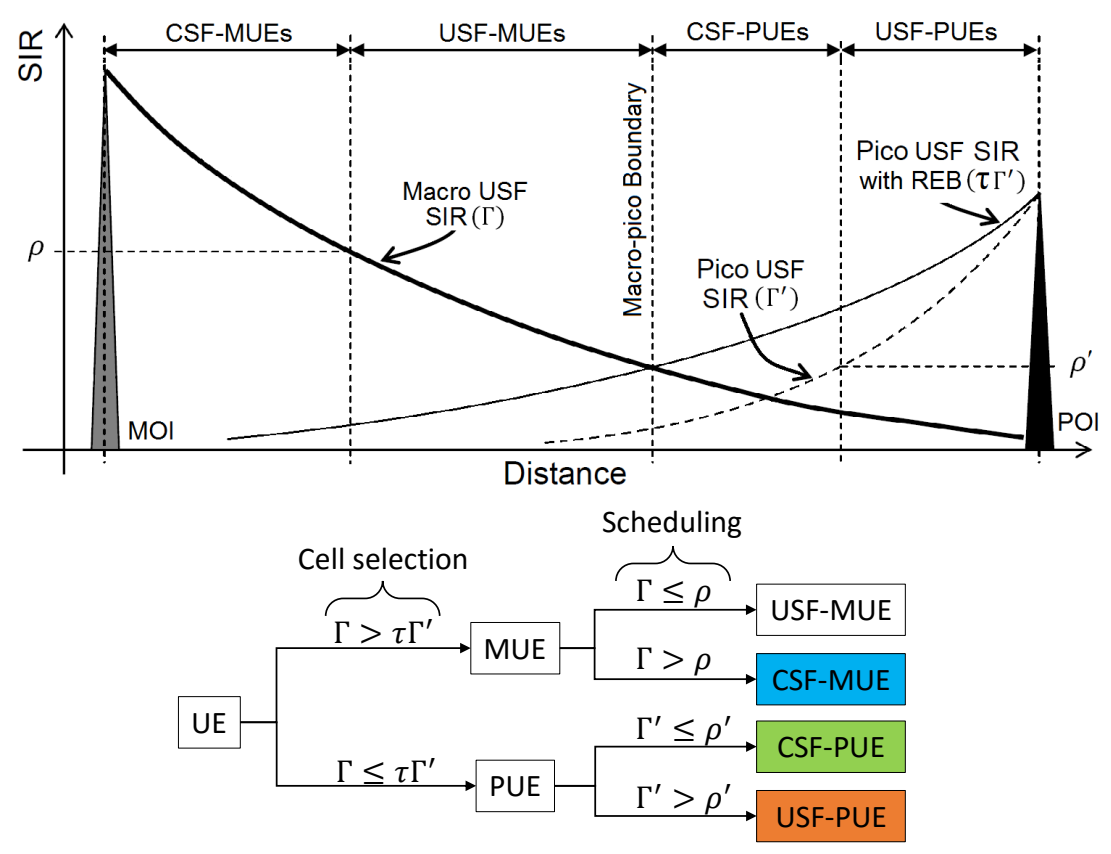

Figure 3.2: Illustration of UE association criteria.

A sample layout of MBSs and PBSs with their coverage areas for the four different UE categories are illustrated in Figure 3.3. Here, the coverage regions for USF- and CSFPUEs in picocells are colored in orange and green, respectively. Whereas in macrocells, the coverage regions for USF- and CSF-MUEs are colored in white and blue, respectively. Note that in the related work of [48], the UE association criteria are based on the average reference symbol received power at UE, where as our model is based on the SIR at UE, it also encompasses the FeICIC mechanism. In [48], the boundary between the USF-PUEs (picocell area) and the CSF-PUEs (range expanded area) is fixed due to the fixed transmit 
power of PBS. On the other hand, in our approach, the boundary between USF and CSF users can be controlled using $\rho$ in macrocell and $\rho^{\prime}$ in picocell, the parameters which play an important role during optimization as will be shown in Section 3.4.3.

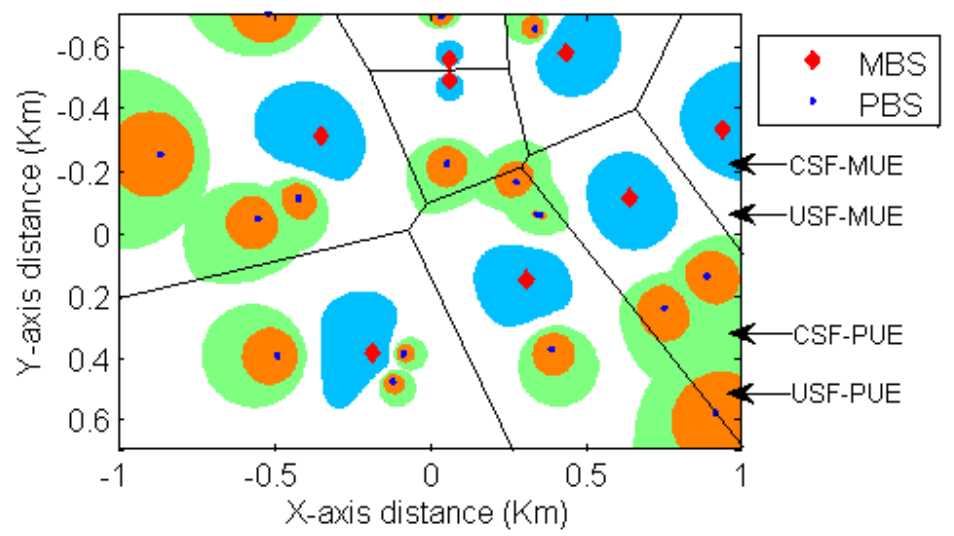

Figure 3.3: Illustration of two-tier HetNet layout.

Using (3.1)-(3.5), it can be shown that the two SIRs $\Gamma_{\text {csf }}$ and $\Gamma_{\text {csf }}^{\prime}$ could be expressed in terms of $\Gamma$ and $\Gamma^{\prime}$ as,

$$
\Gamma_{\mathrm{csf}}=\alpha \Gamma, \quad \Gamma_{\mathrm{csf}}^{\prime}=\frac{\Gamma^{\prime}(1+\Gamma)}{1+\Gamma\left[\alpha\left(\Gamma^{\prime}+1\right)-\Gamma^{\prime}\right]}
$$

Hence, knowing the statistics of $\Gamma$ and $\Gamma^{\prime}$, particularly their joint probability density function (JPDF), would provide a complete picture of the SIR statistics of the HetNet system. We first derive an expression for joint complimentary cumulative distribution function (JCCDF) of $\Gamma$ and $\Gamma^{\prime}$ in Section 3.2.1. Then we differentiate the JCCDF with respect to $\gamma$ and $\gamma^{\prime}$ to get the expression for JPDF in Section 3.2.2, which will then be used for spectral efficiency analysis.

\subsection{Derivation of Joint SIR Distribution}

\subsubsection{JCCDF of $\Gamma$ and $\Gamma^{\prime}$}

From (3.1), we know that $S(r)$ and $S^{\prime}\left(r^{\prime}\right)$ are exponentially distributed with mean $P / r^{\delta}$ and $P^{\prime} /\left(r^{\prime}\right)^{\delta}$, respectively. For brevity, substitute $S(r)=X$ and $S^{\prime}\left(r^{\prime}\right)=Y$ in (3.2) and 


$$
\Gamma=\frac{X}{Y+Z}, \quad \Gamma^{\prime}=\frac{Y}{X+Z}
$$

Using (3.11) it can be easily shown that the product $\Gamma \Gamma^{\prime}$ has a maximum value of 1 .

Let, $R$ and $R^{\prime}$ be the random variables denoting the distances of MOI and POI from a UE. Then, the JCCDF of $\Gamma$ and $\Gamma^{\prime}$ conditioned on $R=r, R^{\prime}=r^{\prime}$ is given by,

$$
\begin{aligned}
& \mathbb{P}\left\{\Gamma>\gamma, \Gamma^{\prime}>\gamma^{\prime} \mid R=r, R^{\prime}=r^{\prime}\right\} \\
& =\mathbb{E}_{Z}\left[\mathbb{P}\left\{X>\gamma(Y+Z), Y>\gamma^{\prime}(X+Z)\right\}\right], \\
& =\mathbb{E}_{Z}\left[\int_{y 1}^{+\infty} f_{Y}(y) \int_{\gamma(y+Z)}^{y / \gamma^{\prime}-Z} f_{X}(x) \mathrm{d} x \mathrm{~d} y\right],
\end{aligned}
$$

for $\gamma>0, \gamma^{\prime}>0, \gamma \gamma^{\prime}<1$. Here, $f_{\mathrm{X}}(x)=\frac{r^{\delta}}{P} \exp \left(-\frac{r^{\delta}}{P} x\right), f_{\mathrm{Y}}(y)=\frac{\left(r^{\prime}\right)^{\delta}}{P^{\prime}} \exp \left(-\frac{\left(r^{\prime}\right)^{\delta}}{P^{\prime}} y\right)$, and the integration limit $y_{1}=\gamma^{\prime} Z\left(\frac{1+\gamma}{1-\gamma \gamma^{\prime}}\right)$. The integration region of (3.12) is graphically represented in Figure 3.4. By solving the integration as shown in Appendix 1, we can obtain a closed form expression for the conditional JCCDF as

$$
\begin{aligned}
& \mathbb{P}\left\{\Gamma>\gamma, \Gamma^{\prime}>\gamma^{\prime} \mid R=r, R^{\prime}=r^{\prime}\right\} \\
& =\frac{\left(1-\gamma \gamma^{\prime}\right) \mathcal{L}_{Z}\left(\frac{1}{1-\gamma \gamma^{\prime}}\left(\frac{\gamma\left(1+\gamma^{\prime}\right) r^{\delta}}{P}+\frac{\gamma^{\prime}(1+\gamma)\left(r^{\prime}\right)^{\delta}}{P^{\prime}}\right)\right)}{\left[1+\gamma \frac{P^{\prime}}{P}\left(\frac{r}{r^{\prime}}\right)^{\delta}\right]\left[1+\gamma^{\prime} \frac{P}{P^{\prime}}\left(\frac{r^{\prime}}{r}\right)^{\delta}\right]},
\end{aligned}
$$

for $\gamma>0, \gamma^{\prime}>0$, and $\gamma \gamma^{\prime}<1$, where $\mathcal{L}_{Z}(s)$ is the Laplace transform of the total interference $Z$.

Expression for $\mathcal{L}_{\mathrm{Z}}(s)$ can be derived as follows. We assume that the interfering MBSs of a UE are frame asynchronous and subframe synchronous. Essentially, we wanted to assume no synchronization at all. However, this would permit part of a subframe from an interfering transmitter to interfere with part of another subframe at the receiver, and the complications for analysis would be too much. To simplify the interference scenario, we would not account for, or model, any interference by partially overlapping subframes. In 


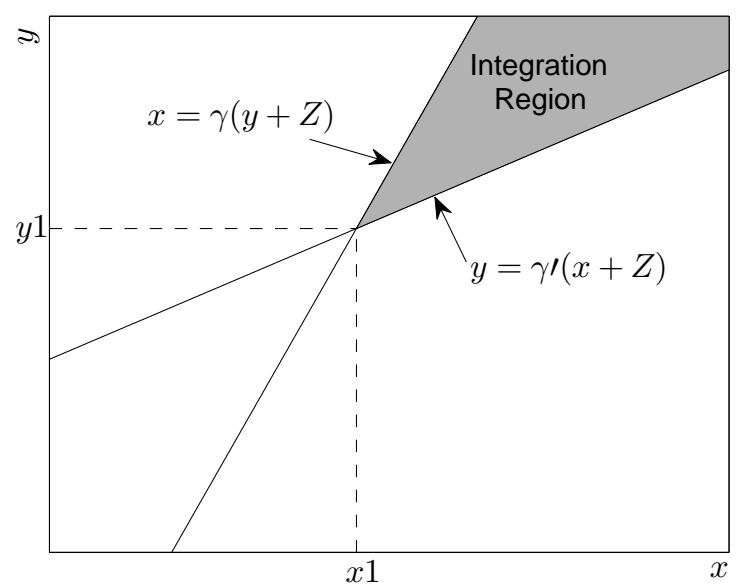

Figure 3.4: Illustration of the integration region in the JPDF of $X$ and $Y$.

other words, if a subframe partially overlaps another subframe, it is assumed to overlap completely. This is equivalent to the "subframe-synchronized but frame-asynchronous" assumption.

The locations of the USFs and CSFs are uniformly randomly distributed, with a USF duty cycle of $\beta$ for all the MBSs. Hence, each interfering MBS transmits USFs with probability $\beta$ and CSFs with probability $(1-\beta)$. Therefore, the tier of MBSs can be split into two tiers, one tier of MBSs transmitting only USFs and other transmitting only CSFs. These two tiers are independent PPPs with intensities $\lambda \beta$ and $\lambda(1-\beta)$. Therefore, the FeICIC scenario can be modeled using three independent PPPs as illustrated in Table 3.1.

Table 3.1: PPP parameters for USF MBSs, CSF MBSs, and PBSs.

\begin{tabular}{|l|l|l|l|l|}
\hline BS type & PPP & Intensity & Tx. power & $\begin{array}{l}\text { Distance of UE } \\
\text { to nearest BS }\end{array}$ \\
\hline USF-MBSs & $\Phi_{\mathrm{usf}}$ & $\beta \lambda$ & $P$ & $r$ \\
\hline CSF-MBSs & $\Phi_{\mathrm{csf}}$ & $(1-\beta) \lambda$ & $\alpha P$ & $r$ \\
\hline PBSs & $\Phi^{\prime}$ & $\lambda^{\prime}$ & $P^{\prime}$ & $r^{\prime}$ \\
\hline
\end{tabular}

Let, $I_{\mathrm{usf}}(r), I_{\mathrm{csf}}(r)$, and $I^{\prime}\left(r^{\prime}\right)$ be the interference at UE from all interfering USF-MBSs, CSF-MBSs and PBSs. Then, the total interference is $Z=I_{\mathrm{usf}}(r)+I_{\mathrm{csf}}(r)+I^{\prime}\left(r^{\prime}\right)$. Using [118, Corollary 1], parameters in Table 3.1, and assuming $\delta=4$, we can derive the Laplace 
transform of $Z$ in (3.13) to be,

$$
\begin{aligned}
\mathcal{L}_{Z}(s)=\exp \{ & -\pi \beta \lambda \sqrt{P s}\left[\frac{\pi}{2}-\tan ^{-1}\left(\frac{r^{2}}{\sqrt{P s}}\right)\right] \\
& -\pi(1-\beta) \lambda \sqrt{\alpha P s}\left[\frac{\pi}{2}-\tan ^{-1}\left(\frac{r^{2}}{\sqrt{\alpha P s}}\right)\right] \\
& \left.-\pi \lambda^{\prime} \sqrt{P^{\prime} s}\left[\frac{\pi}{2}-\tan ^{-1}\left(\frac{\left(r^{\prime}\right)^{2}}{\sqrt{P^{\prime} s}}\right)\right]\right\} .
\end{aligned}
$$

\subsubsection{JPDF of $\Gamma$ and $\Gamma^{\prime}$}

The conditional JPDF of $\Gamma$ and $\Gamma^{\prime}$,

$$
f_{\Gamma, \Gamma^{\prime} \mid R, R^{\prime}}\left(\gamma, \gamma^{\prime} \mid r, r^{\prime}\right)=\mathbb{P}\left\{\Gamma=\gamma, \Gamma^{\prime}=\gamma^{\prime} \mid R=r, R^{\prime}=r^{\prime}\right\}
$$

can be derived by differentiating the JCCDF in (3.13) with respect to $\gamma$ and $\gamma^{\prime}$. Detailed derivation of conditional probability JPDF is provided in Appendix 2. Using the theorem of conditional probability we can write

$$
f_{\Gamma, \Gamma^{\prime}, R, R^{\prime}}\left(\gamma, \gamma^{\prime}, r, r^{\prime}\right)=f_{\Gamma, \Gamma^{\prime} \mid R, R^{\prime}}\left(\gamma, \gamma^{\prime} \mid r, r^{\prime}\right) f_{R}(r) f_{R^{\prime}}\left(r^{\prime}\right)
$$

where, the PDFs of $R$ and $R^{\prime}$ are $f_{R}(r)=2 \pi \lambda r e^{-\lambda \pi r^{2}}$ and $f_{R^{\prime}}\left(r^{\prime}\right)=2 \pi \lambda^{\prime} r^{\prime} e^{-\lambda^{\prime} \pi\left(r^{\prime}\right)^{2}}$, respectively. We can then express the unconditional JPDF of $\Gamma$ and $\Gamma^{\prime}$ as,

$$
\begin{aligned}
f_{\Gamma, \Gamma^{\prime}}\left(\gamma, \gamma^{\prime}\right) & =\int_{d_{\min }}^{\infty} \int_{d_{\min }^{\prime}}^{\infty} f_{\Gamma, \Gamma^{\prime}, R, R^{\prime}}\left(\gamma, \gamma^{\prime}, r, r^{\prime}\right) \mathrm{d} r^{\prime} \mathrm{d} r \\
& =\int_{d_{\min }}^{\infty} \int_{d_{\min }^{\prime}}^{\infty} f_{\Gamma, \Gamma^{\prime}} \mid R, R^{\prime} \\
& \left(\gamma, \gamma^{\prime} \mid r, r^{\prime}\right) f_{R}(r) f_{R^{\prime}}\left(r^{\prime}\right) \mathrm{d} r^{\prime} \mathrm{d} r
\end{aligned}
$$

where, we assume that a UE is served by a BS only if it satisfies the minimum distance constraints: UE should be located at distances of at least $d_{\min }$ from the MOI and $d_{\min }^{\prime}$ from the POI. 


\subsection{Spectral Efficiency Analysis}

In this section, the expressions for aggregate and per-user SEs for different UE categories are derived. Considering the JPDF of an arbitrary UE in (3.17), first the expressions for the probabilities that the UE belongs to each category are derived. Then, these expressions are used to derive the mean number of UEs of each category in a cell. These are followed by the derivation of the aggregate SE. Then, per-user SE expressions are obtained by dividing the aggregate SE by the mean number of UEs.

\subsubsection{MUE and PUE Probabilities}

Depending on the SIRs $\Gamma$ and $\Gamma^{\prime}$, a UE can be one of the four types: USF-MUE, CSF-MUE, USF-PUE or CSF-PUE. Given that the UE is located at a distance $r$ from its MOI and $r^{\prime}$ from its POI, probabilities of the UE belonging to each type can be found by integrating the conditional JPDF over the regions whose boundaries are set by the cell selection conditions in (3.6)-(3.9). Based on these conditions the integration regions for different UE categories are shown in Figure 3.5. Here, the shaded regions indicate the integration regions.

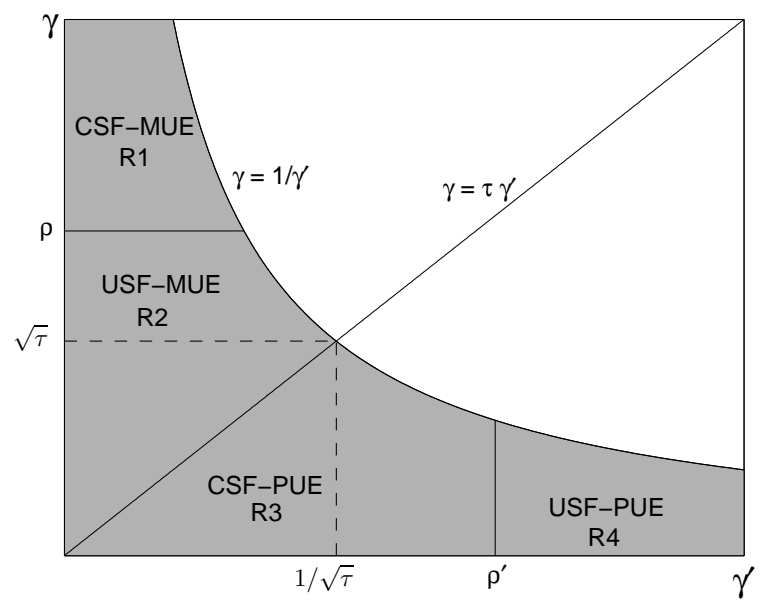

Figure 3.5: Illustration of the integration regions in the JPDF of $\Gamma$ and $\Gamma^{\prime}$. 
The probability that a UE is a CSF-MUE can be found by integrating the JPDF over the region $\mathrm{R} 1$,

$$
P_{\mathrm{csf}}=\mathbb{P}\left\{\Gamma>\tau \Gamma^{\prime}, \Gamma>\rho\right\}=\int_{\rho}^{\infty} \int_{0}^{\min \left(\frac{1}{\gamma}, \frac{\gamma}{\tau}\right)} f_{\Gamma, \Gamma^{\prime}}\left(\gamma, \gamma^{\prime}\right) \mathrm{d} \gamma^{\prime} \mathrm{d} \gamma
$$

To form concise equations, let us define an integral function,

$$
G(g, \mathrm{R} i)=\iint_{\mathrm{R} i} g\left(\gamma, \gamma^{\prime}\right) f_{\Gamma, \Gamma^{\prime}}\left(\gamma, \gamma^{\prime}\right) \mathrm{d} \gamma^{\prime} \mathrm{d} \gamma
$$

where, $g$ is a function of $\gamma$ and $\gamma^{\prime}, \mathrm{R} i$ for $i=1,2,3,4$ is the integration region as defined in Figure 3.5. Then, (3.18) can be written as,

$$
P_{\mathrm{csf}}=\mathbb{P}\left\{\Gamma>\tau \Gamma^{\prime}, \Gamma>\rho\right\}=G(1, \mathrm{R} 1) .
$$

Similarly, the conditional probabilities that a UE is a USF-MUE, USF-PUE or CSF-PUE are respectively given as,

$$
\begin{aligned}
& P_{\mathrm{usf}}=\mathbb{P}\left\{\Gamma>\tau \Gamma^{\prime}, \Gamma \leq \rho\right\}=G(1, \mathrm{R} 2), \\
& P_{\mathrm{usf}}^{\prime}=\mathbb{P}\left\{\Gamma \leq \tau \Gamma^{\prime}, \Gamma^{\prime} \geq \rho^{\prime}\right\}=G(1, \mathrm{R} 4), \\
& P_{\mathrm{csf}}^{\prime}=\mathbb{P}\left\{\Gamma \leq \tau \Gamma^{\prime}, \Gamma^{\prime}<\rho^{\prime}\right\}=G(1, \mathrm{R} 3) .
\end{aligned}
$$

\subsubsection{Mean number of MUEs and PUEs}

Since the MBS locations are generated using PPPs, the coverage areas of all the MBSs resemble a Voronoi tessellation. Consider an arbitrary Voronoi cell. Let the number of UEs in the cell be $N$, and number of CSF-MUEs in the cell be $M$. Then, $M$ is a random variable 
and the mean number of CSF-MUEs is given by,

$$
\begin{aligned}
N_{\mathrm{csf}} & =E[M]=E\left[\sum_{n=1}^{N} 1\{\mathrm{UE} n \text { is a CSF-MUE }\}\right] \\
& =E_{N}\left\{E\left[\sum_{n=1}^{N} 1\{\mathrm{UE} n \text { is a CSF-MUE }\} \mid N\right]\right\} \\
& =E_{N}\left\{\sum_{n=1}^{N} E[1\{\mathrm{UE} n \text { is a CSF-MUE }\}]\right\}
\end{aligned}
$$

where in (3.24) we use the fact that the probability that any of the $N$ UEs in a cell being a CSF-MUE is independent of $N$. However, it is important to note that this is itself a consequence of our assumption that there is no limit on the number of CSF-MUEs per cell. Further, the event that any one of the UEs in a cell is a CSF-MUE is independent of the event that any other UE in that cell is a CSF-MUE, and all such events have the same probability of occurrence, namely $P_{\text {csf }}$ given in (3.20). Then,

$$
N_{\mathrm{csf}}=E_{N}\left\{\sum_{n=1}^{N} P_{\mathrm{csf}}\right\}=E_{N}\left[N P_{\mathrm{csf}}\right]=P_{\mathrm{csf}} E[N]
$$

Using [119, Lemma 1], it can be shown that the mean number of UEs in a Voronoi cell is $\lambda_{\mathrm{u}} / \lambda$. Therefore, the mean number of CSF-MUEs in a cell are given by,

$$
N_{\mathrm{csf}}=\frac{P_{\mathrm{csf}} \lambda_{\mathrm{u}}}{\lambda}
$$

Similarly, the mean number of USF-MUEs, USF-PUEs and CSF-PUEs are respectively given by,

$$
N_{\mathrm{usf}}=\frac{P_{\mathrm{usf}} \lambda_{\mathrm{u}}}{\lambda}, \quad N_{\mathrm{usf}}^{\prime}=\frac{P_{\mathrm{usf}}^{\prime} \lambda_{\mathrm{u}}}{\lambda^{\prime}}, \quad N_{\mathrm{csf}}^{\prime}=\frac{P_{\mathrm{csf}}^{\prime} \lambda_{\mathrm{u}}}{\lambda^{\prime}}
$$




\subsubsection{Aggregate and Per-user Spectral Efficiencies}

We use Shannon capacity formula, $\log _{2}(1+S I R)$, to find the SE of each UE type. The mean aggregate SE of an arbitrarily located CSF-MUE can be found by

$$
\begin{aligned}
C_{\mathrm{csf}}\left(\lambda, \lambda^{\prime}, \tau, \alpha, \rho, \beta\right) & =(1-\beta) \frac{\mathbb{E}\left[\log _{2}\left(1+\Gamma_{\mathrm{csf}}\right) \mid \mathrm{UE} \text { is a CSF-MUE }\right]}{P_{\mathrm{csf}}} \\
& =(1-\beta) \frac{G\left(\log _{2}\left(1+\gamma_{\mathrm{csf}}\right), \mathrm{R} 1\right)}{P_{\mathrm{csf}}} \\
& =(1-\beta) \frac{G\left(\log _{2}(1+\alpha \gamma), \mathrm{R} 1\right)}{P_{\mathrm{csf}}} .
\end{aligned}
$$

Similarly, the mean aggregate SEs for USF-MUEs, USF-PUEs and CSF-PUEs can be respectively derived to be

$$
\begin{aligned}
& C_{\mathrm{usf}}\left(\lambda, \lambda^{\prime}, \tau, \alpha, \rho, \beta\right)=\beta \frac{G\left(\log _{2}(1+\gamma), \mathrm{R} 2\right)}{P_{\mathrm{usf}}}, \\
& C_{\mathrm{usf}}^{\prime}\left(\lambda, \lambda^{\prime}, \tau, \alpha, \rho^{\prime}, \beta\right)=\beta \frac{G\left(\log _{2}\left(1+\gamma^{\prime}\right), \mathrm{R} 4\right)}{P_{\mathrm{usf}}^{\prime}}, \\
& C_{\mathrm{csf}}^{\prime}\left(\lambda, \lambda^{\prime}, \tau, \alpha, \rho^{\prime}, \beta\right)=(1-\beta) \frac{G\left(\log _{2}\left(1+\gamma_{\mathrm{csf}}^{\prime}\right), \mathrm{R} 3\right)}{P_{\mathrm{csf}}^{\prime}},
\end{aligned}
$$

where, $\gamma_{\text {csf }}^{\prime}=\frac{\gamma^{\prime}(1+\gamma)}{1+\gamma\left[\alpha\left(\gamma^{\prime}+1\right)-\gamma^{\prime}\right]}$. Then the corresponding per-user SEs are

$$
\begin{aligned}
C_{\mathrm{u}, \mathrm{usf}}\left(\lambda, \lambda^{\prime}, \tau, \alpha, \rho, \beta\right) & =\frac{\lambda C_{\mathrm{usf}}\left(\lambda, \lambda^{\prime}, \tau, \alpha, \rho, \beta\right)}{\lambda_{\mathrm{u}} P_{\mathrm{usf}}}, \\
C_{\mathrm{u}, \mathrm{csf}}\left(\lambda, \lambda^{\prime}, \tau, \alpha, \rho, \beta\right) & =\frac{\lambda C_{\mathrm{csf}}\left(\lambda, \lambda^{\prime}, \tau, \alpha, \rho, \beta\right)}{\lambda_{\mathrm{u}} P_{\mathrm{csf}}}, \\
C_{\mathrm{u}, \mathrm{usf}}^{\prime}\left(\lambda, \lambda^{\prime}, \tau, \alpha, \rho^{\prime}, \beta\right) & =\frac{\lambda^{\prime} C_{\mathrm{usf}}^{\prime}\left(\lambda, \lambda^{\prime}, \tau, \alpha, \rho^{\prime}, \beta\right)}{\lambda_{\mathrm{u}} P_{\mathrm{usf}}^{\prime}}, \\
C_{\mathrm{u}, \mathrm{csf}}^{\prime}\left(\lambda, \lambda^{\prime}, \tau, \alpha, \rho^{\prime}, \beta\right) & =\frac{\lambda^{\prime} C_{\mathrm{csf}}^{\prime}\left(\lambda, \lambda^{\prime}, \tau, \alpha, \rho^{\prime}, \beta\right)}{\lambda_{\mathrm{u}} P_{\mathrm{csf}}^{\prime}} .
\end{aligned}
$$

\subsubsection{5th Percentile Throughput}

The 5th percentile throughput reflects the throughput of cell-edge UEs. Typically the celledge UEs experience high interference and analyzing their throughput provides important information about the fairness among the users in a cell and the system performance. 
Consider the JPDF expression in (3.17). The integration regions of the JPDF for different UE categories are shown in Figure 3.5. The SIR PDF of USF-MUEs can be evaluated by integrating the JPDF over $\gamma^{\prime}$ in the region $\mathrm{R} 2$,

$$
f_{\Gamma}(\gamma)=\mathbb{P}\{\Gamma=\gamma \mid \text { UE is a USF-MUE }\}=\int_{0}^{\min \left(\frac{\gamma}{\tau}, \frac{1}{\gamma}\right)} f_{\Gamma, \Gamma^{\prime}}\left(\gamma, \gamma^{\prime}\right) \mathrm{d} \gamma^{\prime}
$$

for $0 \leq \gamma \leq \rho$. The cumulative distribution function (CDF) expression can be derived as

$$
\begin{aligned}
\mathrm{F}_{\Gamma}\left(\gamma_{\text {usf }}\right) & =\mathbb{P}\left\{\Gamma \leq \gamma_{\text {usf }} \mid \text { UE is a USF-MUE }\right\} \\
& =\int_{0}^{\gamma_{\text {usf }}} f_{\Gamma}(\gamma) \mathrm{d} \gamma=\int_{0}^{\gamma_{\text {usf }}} \int_{0}^{\min \left(\frac{\gamma}{\tau}, \frac{1}{\gamma}\right)} f_{\Gamma, \Gamma^{\prime}}\left(\gamma, \gamma^{\prime}\right) \mathrm{d} \gamma^{\prime} \mathrm{d} \gamma
\end{aligned}
$$

for $0 \leq \gamma_{\text {usf }} \leq \rho$ and, the CDF of throughput of the USF-MUEs can be derived as a function of $F_{\Gamma}\left(\gamma_{\text {usf }}\right)$ in $(3.37)$ as,

$$
\begin{aligned}
\mathrm{F}_{C_{\mathrm{usf}}\left(c_{\mathrm{usf}}\right)} & =\mathbb{P}\left\{C_{\mathrm{usf}} \leq c_{\mathrm{usf}} \mid \mathrm{UE} \text { is a USF-MUE }\right\} \\
& =\mathbb{P}\left\{\log _{2}\left(1+\Gamma_{\mathrm{usf}}\right) \leq c_{\mathrm{usf}} \mid \text { UE is a USF-MUE }\right\} \\
& =\mathbb{P}\left\{\Gamma_{\mathrm{usf}} \leq\left(2^{c_{\mathrm{usf}}}-1\right) \mid \mathrm{UE} \text { is a USF-MUE }\right\} \\
& =\mathrm{F}_{\Gamma}\left(2^{c_{\mathrm{usf}}}-1\right),
\end{aligned}
$$

for $0 \leq c_{\mathrm{usf}} \leq \log _{2}(1+\rho)$. By using the CDF plots, the 5 th percentile throughput of USFMUEs can easily be found as the value at which the CDF is equal to 0.05. Similarly, the 5th percentile throughput of other three UE categories can also be found.

\subsection{Numerical and Simulation Results}

The average SE and 5th percentile throughput expressions derived in the earlier sections are validated using a Monte Carlo simulation model built in Matlab. Validation of the PPP capacity results for a HetNet scenario with range expansion and reduced power subframes is a non-trivial task. In this section, details of the simulation approach used for validating 
the PPP analyses are explicitly documented to enable reproducibility. Matlab codes for the simulation model and the theoretical analysis can be downloaded from [117].

\subsubsection{Simulation Methodology for verifying PPP Model}

Algorithm used in simulation to find the aggregate and per-user SEs is described below.

1. The X-and Y-coordinates of MBSs, PBSs and UEs are generated using uniformly distributed random variables. The mean number of MBS and PBS location marks are $\lambda A$ and $\lambda^{\prime} A$ respectively, where, $A$ is the assumed geographical area that is square in shape as illustrated in Figure 3.6.

2. In the PPP analysis, the geographical area is assumed to be infinite. In such case, it is important to account for edge effects in the simulations. In a tessellation that is defined on an unbounded region, what happens outside a bounded simulation window may effect what happens within the window [120]. As the simulation area is limited, if a UE is located at the edge of the simulation area, the BSs around it will not be symmetrically distributed. Hence, to avoid the edge effects the UE locations are constrained within a smaller area $A_{\mathrm{u}}$ that is aligned at center of the main simulation area $A$ to avoid the UEs from being located at the edges. The mean number of UEs in the area $A_{\mathrm{u}}$ is $\lambda_{\mathrm{u}} A_{\mathrm{u}}$.

3. The MOI (closest MBS) and POI (closest PBS) for each UE is identified. The minimum distance constraints are applied by discarding the UEs that are closer than $d_{\min }\left(d_{\min }^{\prime}\right)$ from their respective MOIs (POIs).

4. The SIRs $\Gamma, \Gamma^{\prime}, \Gamma_{\mathrm{csf}}, \Gamma_{\mathrm{csf}}^{\prime}$ are calculated for each UE using (3.2)-(3.5).

5. The UEs are classified as USF-MUEs, CSF-MUEs, USF-PUEs and CSF-PUEs using the conditions in (3.6)-(3.9).

6. The MUEs (PUEs) which share the same MOI (POI) are grouped together to form the macro- and pico-cells. 
7. The SEs of all the UEs are calculated. In a cell, SE of a USF-MUE $i$ is calculated using $\frac{\beta \log _{2}\left(1+\Gamma_{i}\right)}{(\text { No. of USF-MUEs in the cell) }}$. The SEs of other UE types are calculated using similar formulations.

8. The aggregate capacity of each UE type is calculated in all the cells.

9. Mean aggregate capacity and mean number of UEs of each type are calculated by averaging over all the cells.

10. The per-user SE of each UE type are calculated by (mean aggregate capacity)/(mean number of UEs).

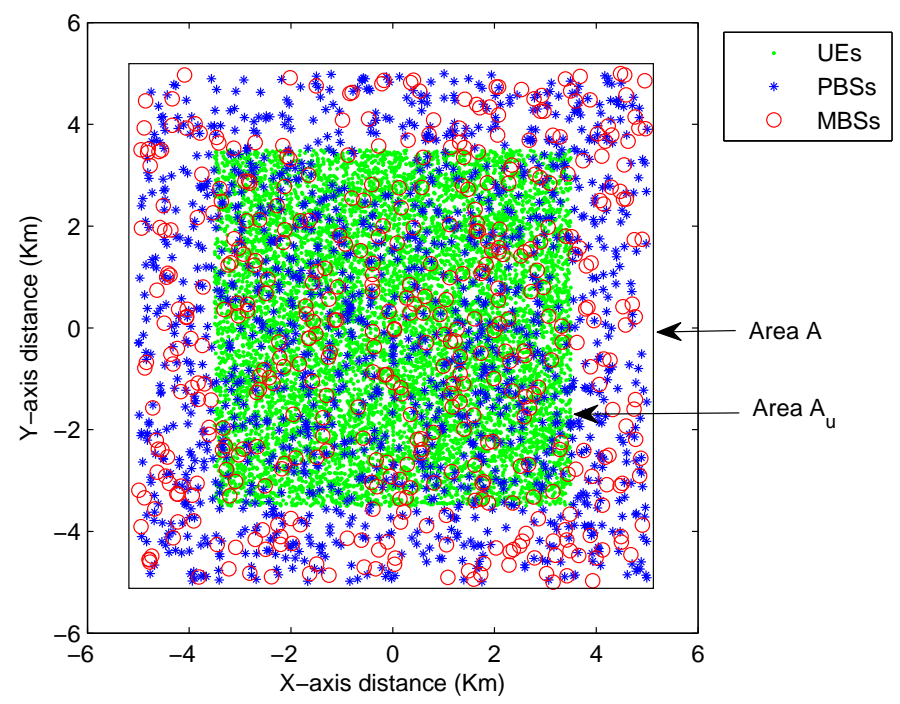

Figure 3.6: Simulation layout.

\subsubsection{Per-user SEs with PPPs and Monte Carlo Simulations}

The system parameter settings are shown in Table 3.2. The per-user SE results obtained using the analytic expressions of (3.32)-(3.35) are compared with the simulation results in Figures 3.7(a) and 3.7(b) for macrocell and picocell, respectively. The averaging process in the simulations is not straight forward and it can be explained as follows. With reference to Figure 3.6, the inner simulation area $A_{\mathrm{u}}$ where the UEs are distributed consists of random number of macrocells and picocells in each simulation instance. On average, it contains 
$\lambda A_{\mathrm{u}}$ macrocells and $\lambda^{\prime} A_{\mathrm{u}}$ picocells. Since the simulation results are obtained by averaging over the macrocells and picocells, we can say that the simulation results were obtained by averaging over approximately $\lambda A_{\mathrm{u}} N_{\text {sim }}$ macrocells and $\lambda^{\prime} A_{\mathrm{u}} N_{\text {sim }}$ picocells, where $N_{\text {sim }}$ is the number of simulation instances. Using the parameter values in Table 3.2 and $N_{\text {sim }}=20$, we can say that the simulation results were obtained by averaging over approximately 4,508 macrocells and 13,524 picocells.

Table 3.2: Parameter settings.

\begin{tabular}{|l|l|}
\hline$P, P^{\prime}$ & $46 \mathrm{dBm}, 30 \mathrm{dBm}$ \\
\hline$K, K^{\prime}$ & $-11 \mathrm{dBm}$ \\
\hline$d_{\min }, d_{\min }^{\prime}$ & $35 \mathrm{~m}, 10 \mathrm{~m}$ \\
\hline$\lambda, \lambda^{\prime}, \lambda_{\mathrm{u}}\left(\right.$ marks $\left./ \mathrm{Km}^{2}\right)$ & $4.6,3 \lambda, 200$ \\
\hline Fading model, Path-loss exponent $(\delta)$ & Rayleigh, 4 \\
\hline$\beta, \rho, \rho^{\prime}$ & $0.5,4 \mathrm{~dB}, 0 \mathrm{~dB}$ \\
\hline$A, A_{\mathrm{u}}$ & $10 \times 10 \mathrm{~km}^{2}, 7 \times 7 \mathrm{~km}^{2}$ \\
\hline
\end{tabular}

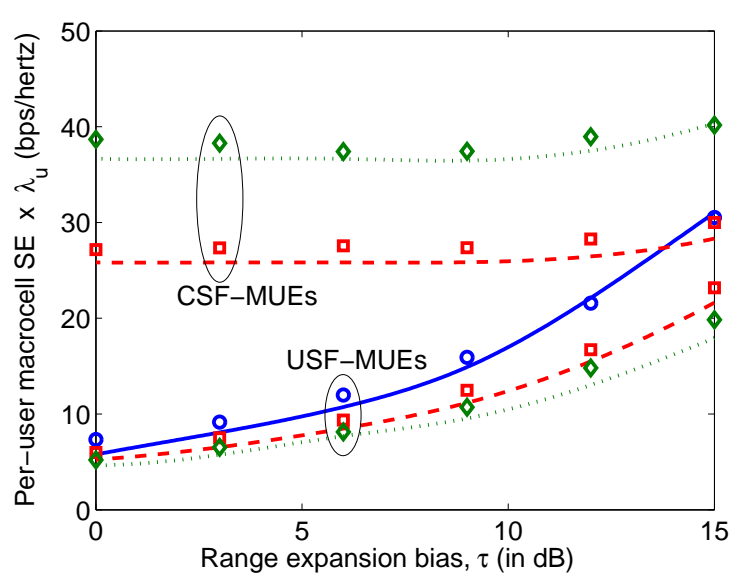

(a) Per-user SE in macrocell.

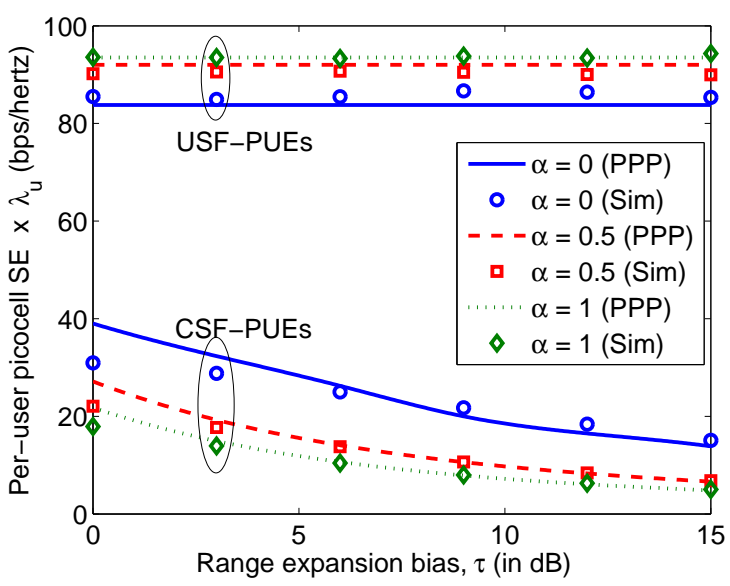

(b) Per-user SE in picocell.

Figure 3.7: Per-user SE in macrocell and picocell with $\beta=0.5, \rho=4 \mathrm{~dB}$ and $\rho^{\prime}=0 \mathrm{~dB}$.

The analytic and simulation plots in Figures 3.7(a) and 3.7(b) match with sufficient accuracy. However, there exists a slight disagreement between the analytic and simulation results which could be due to the fact that the calculation of analytic results involves four nested integrals. Since the numerical integration in Matlab has certain tolerance limits, the results could be off the ideal values. Another source for disagreement could be due to the fact that in theoretical analysis, the BSs are assumed to be distributed over an infinite 
geographical area. However, the simulations are performed using a finite area of $10 \times 10$ $\mathrm{km}^{2}$. Nevertheless, Figure 3.7 provides the following insights:

\subsubsection{USF- and CSF-MUEs}

Referring to Figure 3.2, USF-MUEs form the outer part and CSF-MUEs form the inner part of the macrocell. As the REB increases, some of the USF-MUEs at the macro-pico boundary which have worse SIRs are offloaded to the picocell. Consequently, the mean number of USF-MUEs decreases and their per-user SE increases as shown in Figure 3.7(a).

The mean number of CSF-MUEs are not affected by $\tau$ as long as $\sqrt{\tau} \leq \rho$. Considering Figure 3.5, it can be noted that if $\sqrt{\tau}=\rho$, the line $\gamma=\tau \gamma^{\prime}$ intersects the boundary of region R1. Hence, if $\tau$ is increased further such that $\sqrt{\tau}>\rho$, the area of R1 decreases and thereby decreases the mean number of CSF-MUEs. Therefore, the per-user SE of CSFMUEs remains constant as long as $\sqrt{\tau} \leq \rho$, and increases if $\tau$ crosses this limit as shown in Figure 3.7(a).

On the other hand, as the $\alpha$ increases, the transmit power of all the interfering MBSs increases during CSFs, hence it increases the interference power $Z$ at all the UEs. This causes the SIRs of USF-MUEs $(\Gamma)$, USF-PUEs $\left(\Gamma^{\prime}\right)$ and CSF-PUEs $\left(\Gamma_{\text {csf }}^{\prime}\right)$ to decrease, which can be noted in (3.2), (3.3), and (3.5), respectively. However, the SIRs of CSF-MUEs $\left(\Gamma_{\mathrm{csf}}\right)$ would increase (despite of increased interference) because of the increase in received signal power (due to higher $\alpha$ ) which can be noted in (3.4). Considering (3.6) and (3.7), since $\rho$ is a constant, the degradation in $\Gamma$ causes the number of USF-MUEs to increase and CSF-MUEs to decrease. Consequently, the per-user SE of USF-MUEs decreases and that of CSF-MUEs increases for increasing $\alpha$, as shown in Figure 3.7(a).

\subsubsection{USF- and CSF-PUEs}

As the REB increases, the mean number of USF-PUEs remains constant if $\rho^{\prime}>1 / \sqrt{\tau}$ because the area of region R4 in Figure 3.5 is unaffected by the value of $\tau$. Therefore, the peruser SE of USF-PUEs also remain constant for increasing REB as shown in Figure 3.7(b). With increasing REB, some MUEs are offloaded to the picocell and become CSF-PUEs. 
But, these UEs are located at cell-edges and have low SIRs. Hence the per-user SE of CSF-PUEs decreases as shown in Figure 3.7(b).

On the other hand, as the $\alpha$ increases, the transmit power of all the interfering MBSs increases during CSFs causing $\Gamma, \Gamma^{\prime}$ and $\Gamma_{\mathrm{csf}}^{\prime}$ to decrease and $\Gamma_{\mathrm{csf}}$ to increase, as explained previously. Considering (3.8) and (3.9), since $\rho^{\prime}$ is a constant the degradation in $\Gamma^{\prime}$ causes the number of USF-PUEs to decrease and CSF-PUEs to increase. Consequently, the peruser SE of USF-PUEs increases and that of CSF-PUEs decreases for increasing $\alpha$, as shown in Figure 3.7(b).

\subsubsection{Optimization of System Parameters to Achieve Maximum Capacity and Proportional Fairness}

The five parameters $\tau, \alpha, \beta, \rho$, and $\rho^{\prime}$ are the key system parameters that are critical to the satisfactory performance of the HetNet system. The goal of these parameter settings is to maximize the aggregate capacity in a cell while providing proportional fairness among the users.

Consider an arbitrary cell which consists of $N$ UEs. Let $C_{i}$ be the capacity of an arbitrary UE $i \in\{1,2, \ldots, N\}$. The sum of capacities (sum-rate) and the sum of $\log$ capacities (log-rate) in a cell are respectively given by,

$$
C_{\text {sum }}=\sum_{i=1}^{N} C_{i}, \quad C_{\log }=\sum_{i=1}^{N} \log \left(C_{i}\right)=\log \left(\prod_{i=1}^{N} C_{i}\right)
$$

Maximizing the $C_{\text {sum }}$ corresponds to maximizing the aggregate capacity in a cell, while maximizing the $C_{\log }$ corresponds to proportional fair resource allocation to the users of a cell [121, App. A], [122]. There can be trade-offs existing between aggregate capacity and fairness in a cell. Maximizing the $C_{\text {sum }}$ may reduce the $C_{\log }$, and vice versa. In this section, we try to understand these trade-offs by analyzing the characteristics of $C_{\log }$ and $C_{\text {sum }}$ with respect to the variation of key system parameters.

We attempt to maximize the aggregate capacity and the proportional fairness among the users by jointly optimizing the five key system parameters which can be mathematically 
formulated as,

$$
\begin{gathered}
\max _{\rho, \rho^{\prime}, \alpha, \tau, \beta} C_{\mathrm{sum}}=\max _{\rho, \rho^{\prime}, \alpha, \tau, \beta} \sum_{i=1}^{N} C_{i}, \\
\text { and, } \quad \max _{\rho, \rho^{\prime}, \alpha, \tau, \beta} C_{\log }=\max _{\rho, \rho^{\prime}, \alpha, \tau, \beta} \log \left(\prod_{i=1}^{N} C_{i}\right) .
\end{gathered}
$$

We solve the optimization problem numerically with brute-force search technique. As there are five optimization parameters, this problem involves searching for an optimum solution in a five-dimensional space. The variation of $C_{\log }$ with respect to $\rho, \rho^{\prime}, \alpha, \tau$ is shown in Figure 3.8, for $\beta=0.5$. These plots are obtained through the Monte Carlo simulations and each plot is the variation of $C_{\log }$ with respect to $\rho$ for fixed values of $\rho^{\prime}, \alpha$ and $\tau$. The optimum scheduling thresholds $\rho^{*}$ and $\rho^{*}$ that maximize the $C_{\log }$ are dependent on the values of $\alpha$ and $\tau$.
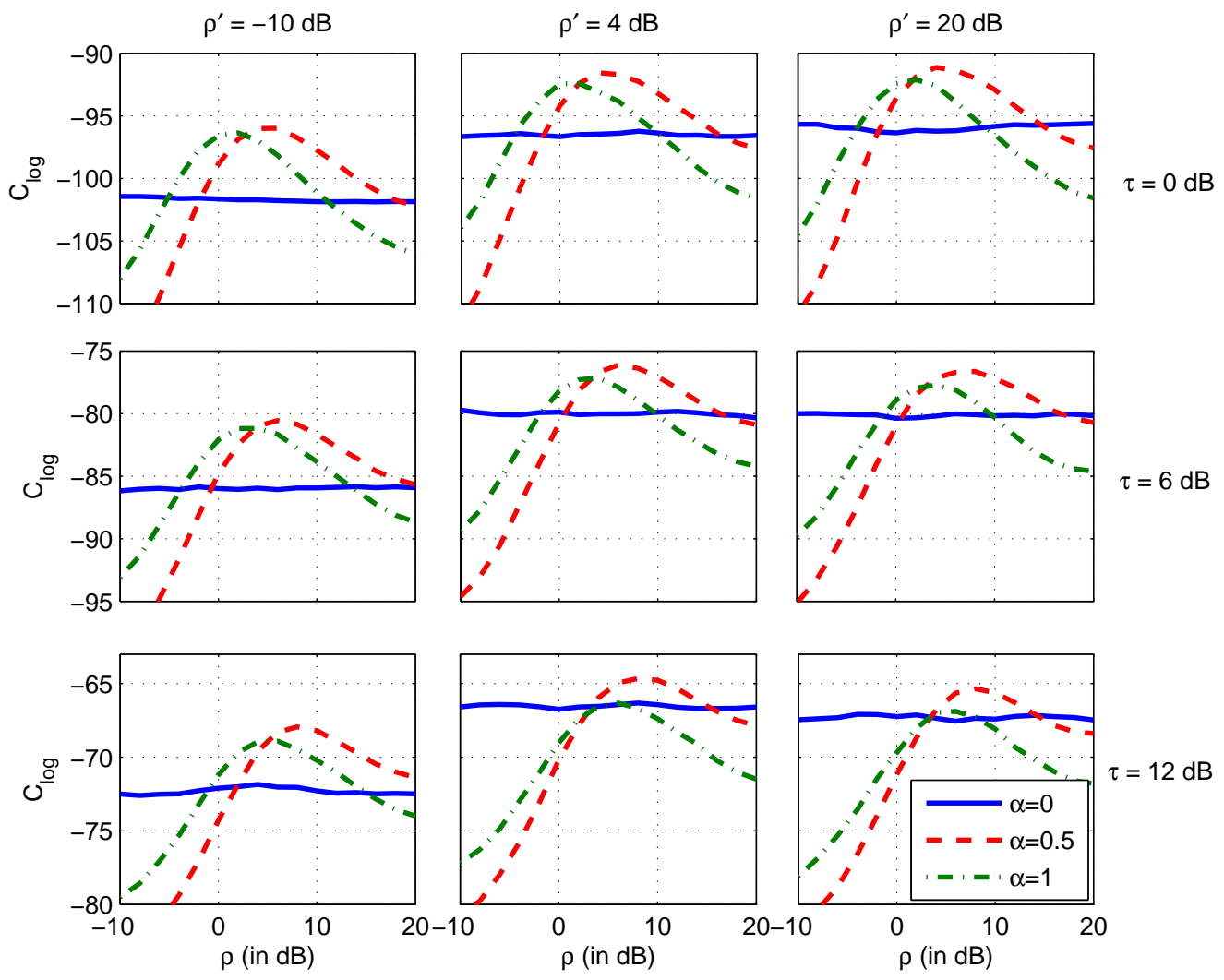

Figure 3.8: Sum of log capacities versus the scheduling thresholds for different $\alpha$ and $\tau$ combinations. 
We have used a simple brute force search technique to optimize the system parameters, while it is also possible to use non-linear optimization techniques. For example, reinforcement learning method is used in $[123,124]$ to optimize the downlink transmission strategies in HetNets such as the transmit power and the REB. In [108], a game theoretic approach and distributed learning algorithm is used to optimize the downlink transmit power, REB, and the ON/OFF states, of individual BSs to minimize the system cost which includes energy and load expenditures. Typically, these optimization techniques use distributed approach and are developed to be efficient from the implementation perspective. In addition, some information exchange among the BSs are typically required for these optimization methods to work. For example, in [108], estimated traffic load, transmission power, and REB are broadcasted by the BSs for optimization of the operating parameters at each individual small cell BS. On the other hand, the brute force search technique does not require any information exchange among the BSs. In this research, our focus is to understand the characteristics of the optimum system parameters, rather than the implementation efficiency of the optimization method used. Brute force search method is also used, for example, in [48] to find the optimum REB and duty cycle of almost blank subframes that maximize the rate coverage in HetNets.

Figure 3.9 shows the plots of $\rho^{*}$ and $\rho^{\prime *}$ as the functions of $\alpha$ and $\tau$. The markers show the simulation results while the dotted lines show the smoother estimation obtained using the curve fitting tool in MATLAB. For small $\alpha$ values, the optimum threshold $\rho^{*}$ has higher values as shown in Figure 3.9(a), and according to (3.7) this causes very few MUEs that have $\Gamma>\rho^{*}$ to be scheduled during CSFs. This makes sense because MBS transmit power during CSFs is very low for small $\alpha$ and hence the number of CSF-MUEs which can be covered is also less. On the other hand, for higher $\alpha$ values, MBS transmits with higher power level during CSFs and can cover larger number of CSF-MUEs. Therefore, to improve the fairness proportionally, the optimal $\rho^{*}$ value decreases with increasing $\alpha$ so that more MUEs are scheduled during CSFs.

In the picocell, with increasing $\alpha$ the CSF-PUEs at the cell edges will experience higher interference from the MBSs. Then, more PUEs should be scheduled during USFs to improve 

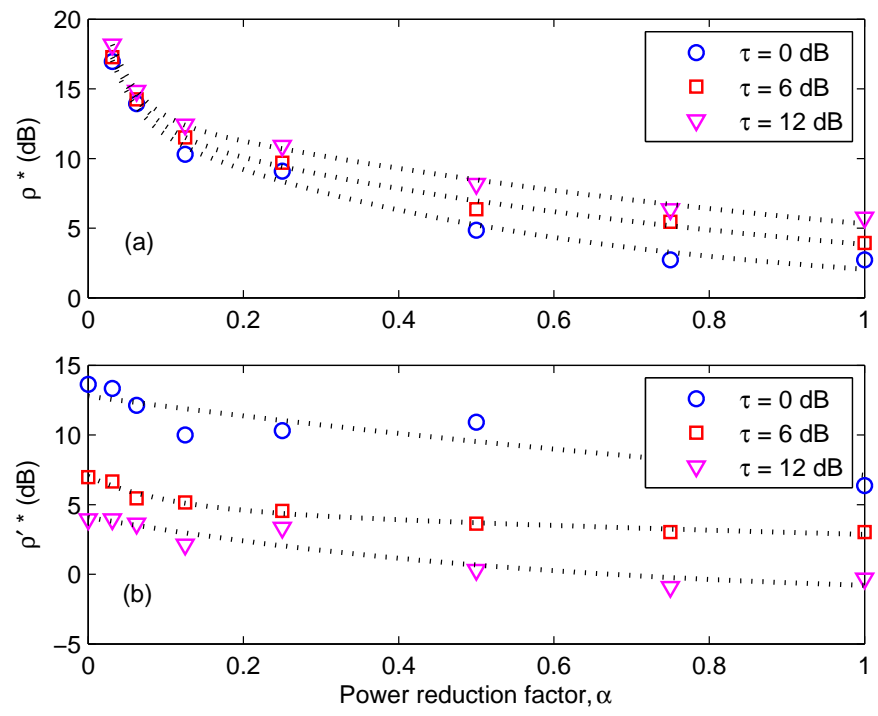

Figure 3.9: Optimized scheduling thresholds versus $\alpha$ for different $\tau$ (a) in macrocell; (b) in picocell. With $\lambda=4.6$ marks $/ \mathrm{Km}^{2}$ and $\lambda^{\prime}=13.8$ marks $/ \mathrm{Km}^{2}$.

proportional fairness. Likewise, decreasing $\rho^{\prime *}$ in Figure 3.9(b) indicates that more PUEs are scheduled during USFs as per (3.8).

The $C_{\log }$ with optimum scheduling thresholds $\rho^{*}$ and $\rho^{* *}$ is plotted in Figure 3.10(a). Higher the $C_{\log }$, better is the proportional fairness. It is important to note that the range expansion bias, $\tau$, has a significant effect on proportional fairness. The $C_{\log }$ increases from -40 to -28 when $\tau$ is increased from $0 \mathrm{db}$ to $12 \mathrm{~dB}$.

Compared to $\tau, \alpha$ has a smaller effect on the proportional fairness. When $\alpha$ is set to zero which corresponds to the eICIC, $C_{\log }$ is at its minimum. It shows that eICIC provides minimum proportional fairness. Figure 3.10(a) moreover shows that setting $\alpha=1$ which corresponds to no eICIC, also does not provide maximum $C_{\log }$. An $\alpha$ setting between 0.125 and 0.5 maximizes the $C_{\log }$ and hence the proportional fairness.

The characteristics of $C_{\text {sum }}$ with optimum scheduling thresholds is shown in Figure 3.10b. As the $\tau$ increases, $C_{\text {sum }}$ decreases, which is the opposite effect when compared to the $C_{\log }$ in Figure 3.10(a). This shows the trade-off between the aggregate capacity and the 


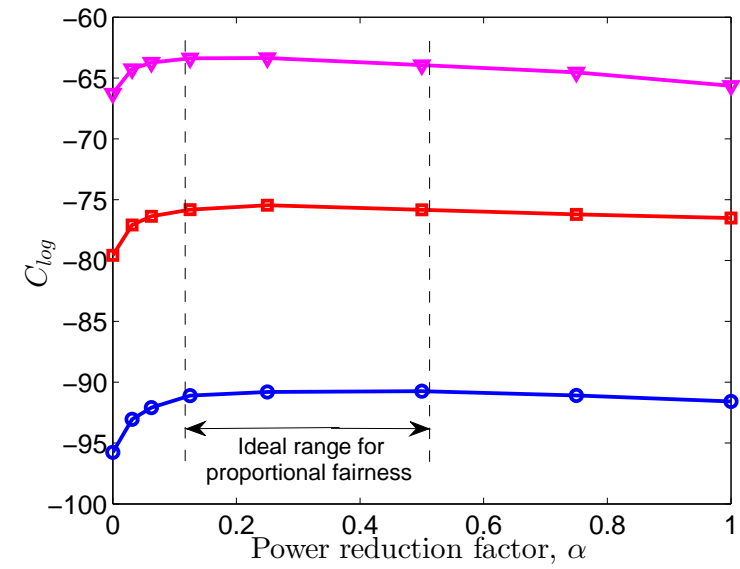

(a) $C_{\log }$ versus $\alpha$

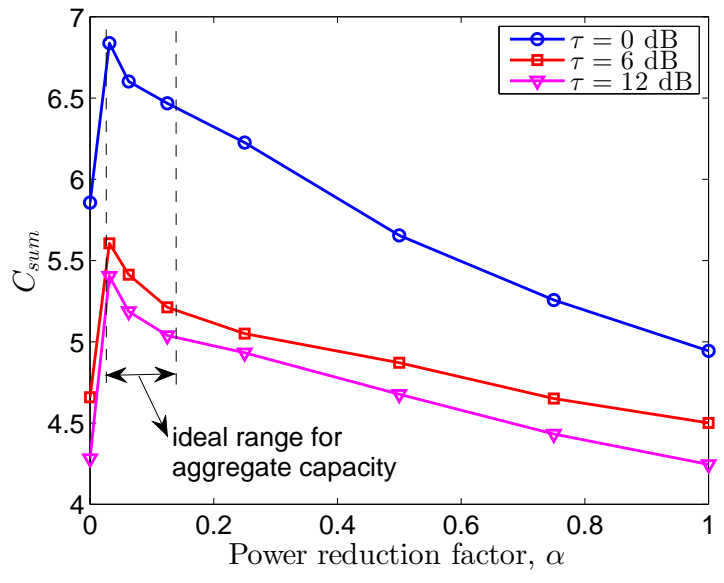

(b) $C_{\text {sum }}$ versus $\alpha$

Figure 3.10: $C_{\log }$ and $C_{\text {sum }}$ with optimum scheduling thresholds $\rho^{*}$ and $\rho^{\prime *} ; \lambda=$ 4.6 marks $/ \mathrm{Km}^{2}$ and $\lambda^{\prime}=13.8$ marks $/ \mathrm{Km}^{2}$.

proportional fairness. Increasing the $\tau$ would increase the proportional fairness but decrease the aggregate capacity, and vice versa.

Comparing Figures 3.10(a) and 3.10(b) also explains the trade-off associated with setting $\alpha$. A very small value, $0<\alpha<0.125$, provides larger $C_{\text {sum }}$ but smaller $C_{\log }$, which is better from an aggregate capacity point of view. Setting $0.125 \leq \alpha \leq 0.5$ is better from a fairness point of view. Any value of $\alpha>0.5$ is not recommended since it degrades the aggregate capacity as shown in Figure 3.10(b), decreases the proportional fairness as shown in Figure 3.10(a), and consumes higher transmit power by the MBSs. Setting $\alpha=0$ as in the eICIC case would reduce both $C_{\text {sum }}$ and $C_{\log }$ drastically.

The effects of $\alpha$ and $\tau$ on the 5th percentile, 50th percentile and average SEs are shown in Figure 3.11. Here again, optimum scheduling thresholds $\rho^{*}$ and $\rho^{* *}$ are used. Figure 3.11(a) shows that as the REB increases from $0 \mathrm{~dB}$ to $6 \mathrm{~dB}$, some of the MUEs at the border of the macrocell are offloaded to the picocell. Since these offloaded UEs are served by picocell during the CSFs, they would have better throughput resulting into the improvement in 5th percentile SE. However, if the REB increases to $12 \mathrm{~dB}$, more MUEs are offloaded and the picocell becomes crowded resulting into poor SEs for the PUEs. Hence the 5th percentile SE decreases when the REB increases from $6 \mathrm{~dB}$ to $12 \mathrm{~dB}$. Figure 3.11(a) also 

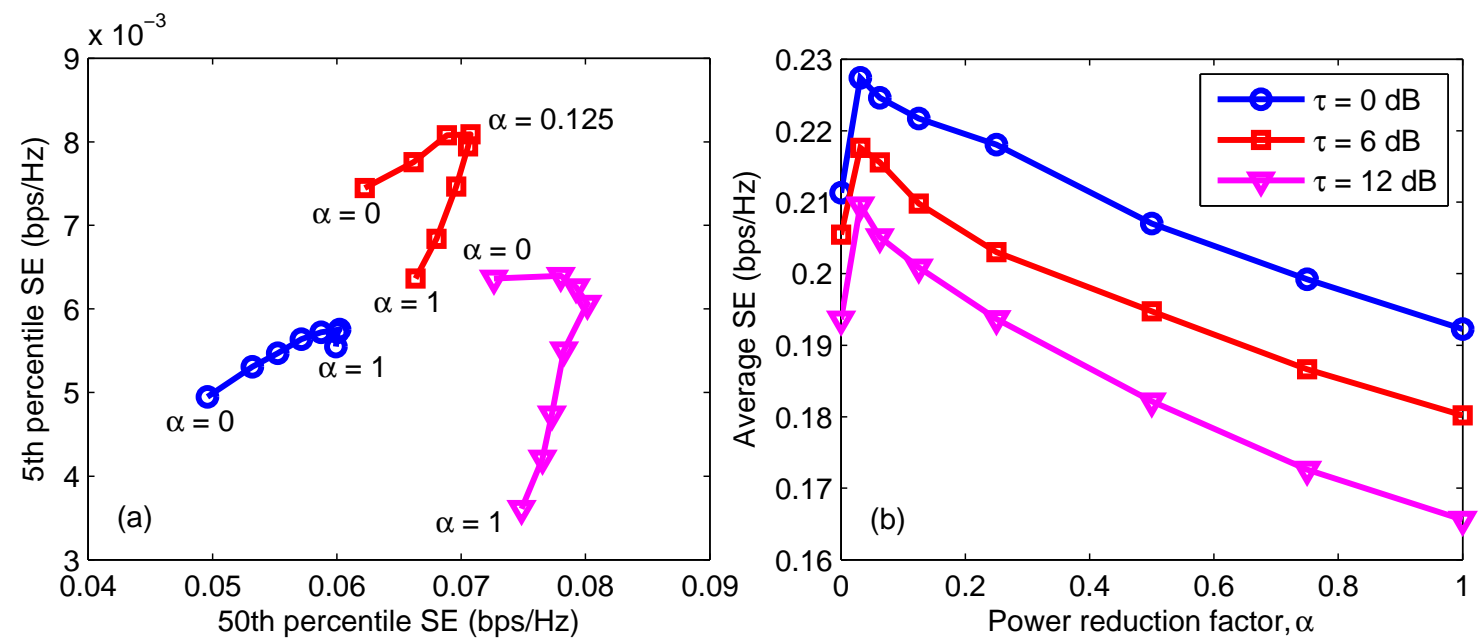

Figure 3.11: Effects of $\alpha$ and $\tau$ on the 5 th percentile, 50th percentile, and average SEs. (a) 5 th percentile SE versus 50th percentile SE; (b) Average SE versus power reduction factor $\alpha$. With $\lambda=4.6$ marks $/ \mathrm{Km}^{2}$ and $\lambda^{\prime}=13.8$ marks $/ \mathrm{Km}^{2}$.

shows that with $\tau=6 \mathrm{~dB}$, setting $\alpha=0.125$ maximizes both 5 th and 50 th percentile SEs. Figure 3.11(b) shows the characteristics of average SE of an arbitrary UE, which is similar to the characteristics of $C_{\text {sum }}$ in Figure 3.10(b). By comparing Figures 3.11(a) and 3.11(b), it can be noted that the 50th percentile SE and the average SE have opposite behaviors with respect to the REB. As the REB increases, 50th percentile SE increases while the average SE decreases.

\subsubsection{Impact of the Duty Cycle of Uncoordinated Subframes}

In the results of Figures 3.9-3.11, $\beta$ was set to 0.5 and we next show the effect of varying $\beta$ on $C_{\log }$ and $C_{\text {sum. }}$. Introducing $\beta$ into the optimization problem makes it difficult to visualize the results due to the addition of one more dimension. Therefore, we use the optimized scheduling thresholds, $\rho^{*}$ and $\rho^{\prime *}$, and analyze $C_{\log }$ and $C_{\text {sum }}$ as the functions $\beta$, $\alpha$ and $\tau$. Figures 3.12 and 3.13 show the $C_{\log }$ versus $\beta$ and the $C_{\text {sum }}$ versus $\beta$, respectively for different values of $\alpha$ and $\tau$. The variation of $C_{\log }$ with respect to $\beta$ is not significant, except for $\alpha=0$. Whereas, the variation of $C_{\text {sum }}$ with respect to $\beta$ is significant.

When $\alpha=0$, the $C_{\log }$ value decreases rapidly for $\beta<0.5$. Nevertheless, $\alpha=0$ is shown to have poor performance in the previous paragraphs and hence it is not recommended. 


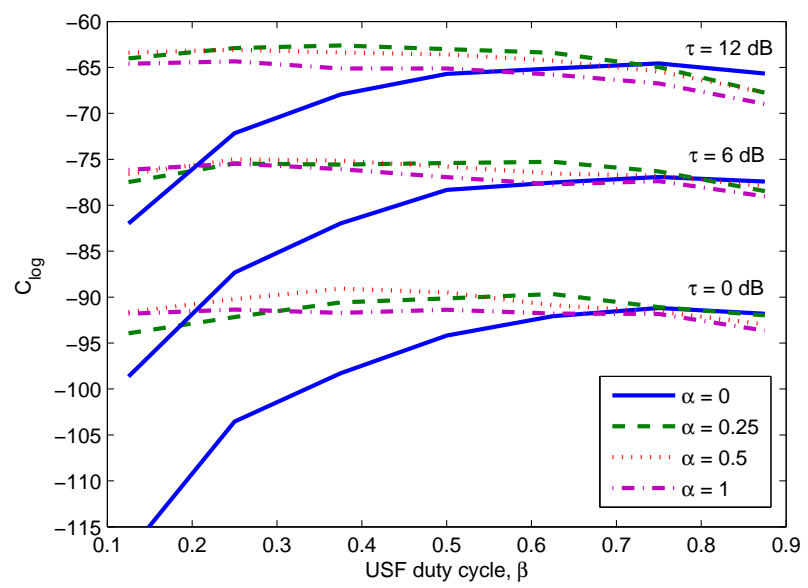

Figure 3.12: $C_{\log }$ versus $\beta$ with optimum scheduling thresholds $\rho^{*}$ and $\rho^{*}$. With $\lambda=$ 4.6 marks $/ \mathrm{Km}^{2}$ and $\lambda^{\prime}=13.8$ marks $/ \mathrm{Km}^{2}$.
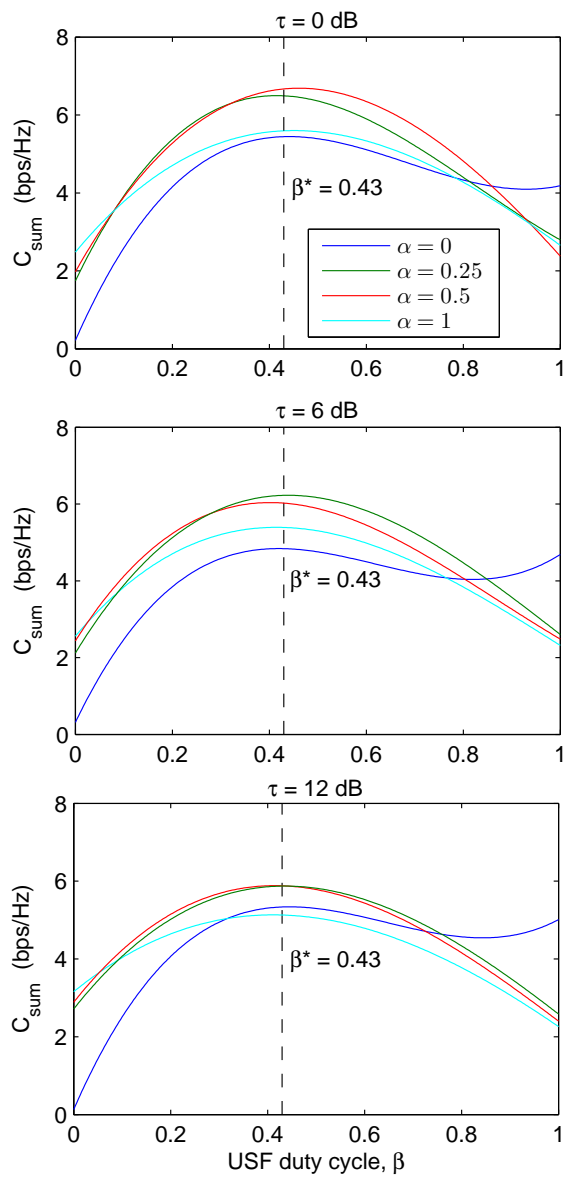

Figure 3.13: $C_{\text {sum }}$ versus $\beta$ with optimum scheduling thresholds $\rho^{*}$ and $\rho^{\prime *}$. With $\lambda=$ 4.6 marks $/ \mathrm{Km}^{2}$ and $\lambda^{\prime}=13.8$ marks $/ \mathrm{Km}^{2}$. 
For other values of $\alpha$, variation in $\beta$ does not affect the $C_{\log }$ significantly, which shows that by using a fixed value of $\beta$, proportional fairness can be achieved by optimizing (to maximize $C_{\log }$ ) the scheduling thresholds. Figure 3.13 shows that fixing $\beta$ approximately to 0.43 maximizes the $C_{\text {sum }}$ irrespective of $\alpha$ and $\tau$, provided the scheduling thresholds are optimized to maximize $C_{\log }$.

In [48], the boundary of CSF-PUEs that form the inner region of picocell (excluding the range expansion region) is fixed due to the fixed transmit power of PBS. The association bias and resource partitioning fraction parameters are used as the variables to be optimized. It is analogous for us to have a fixed $\rho^{\prime}$ and optimize $\beta$ and $\tau$. But in contrast, we fix the $\beta$ for simplicity and optimize the other four parameters, since coordinating $\beta$ among the cells through the X2 interface is complex and adds to communication overhead in the backhaul. The X2 is a type of interface in LTE networks which connects neighboring eNodeBs in a peer to peer fashion to assist handover and provide a means for rapid coordination of radio resources [125].

\subsubsection{5th Percentile Throughput}

Using the expressions derived in Section 3.3.4, the 5th percentile throughput versus $\alpha$ for different $\tau$ is shown in Figure 3.14(a) for MUEs, and in Figure 3.14(b) for PUEs. As the $\alpha$ increases, MBSs transmit at higher power level during CSFs and the UEs of all types experience a higher interference power. However, the received signal power at CSFMUEs increases with $\alpha$ and results in improved 5th percentile throughput as shown in Figure 3.14(a). But, the SIRs of USF-MUEs and USF/CSF-PUEs degrade due to higher interference and therefore their 5th percentile throughput decreases with increase in $\alpha$ as shown in Figures 3.14(a) and 3.14(b).

Increasing the REB, $\tau$, causes the USF-MUEs with poor SIR, located at the edge of macrocell, to be offloaded to the picocell and thereby increasing the 5th percentile throughput of USF-MUEs as shown in Figure 3.14(a). The offloaded UEs in picocell are scheduled

during CSFs and due to their poor SIR the 5th percentile throughput of CSF-PUEs decreases as shown in Figure 3.14(b). 

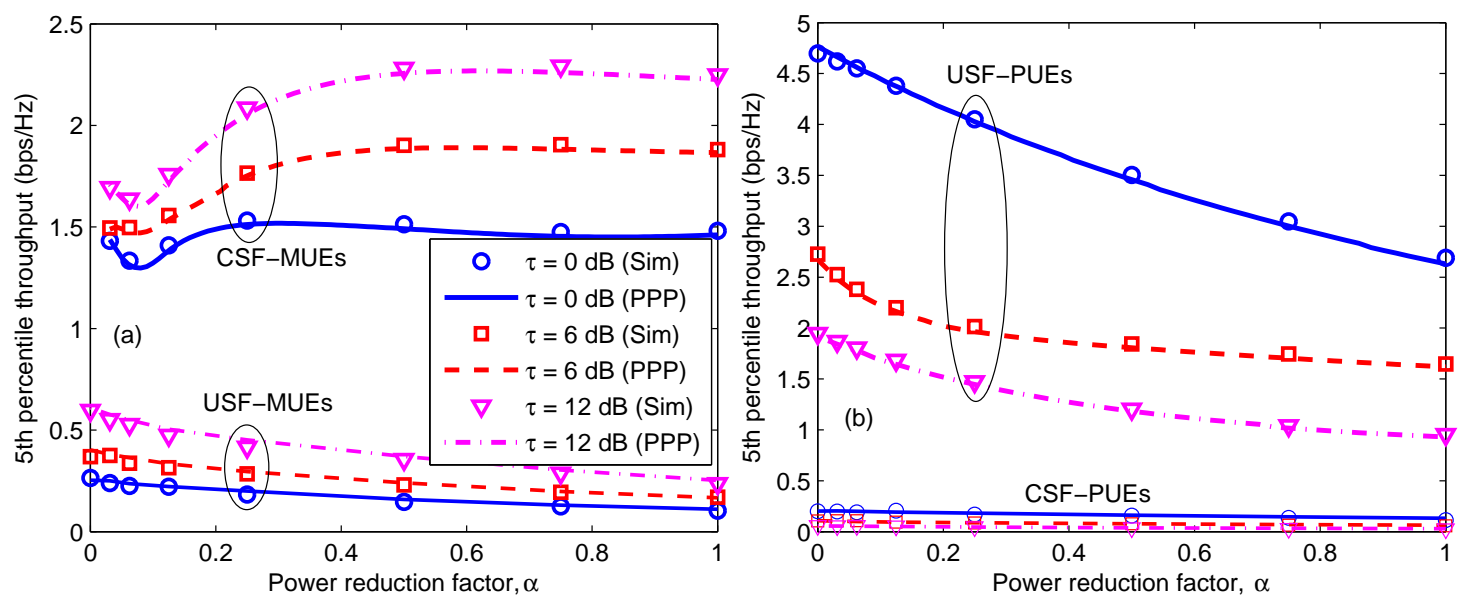

Figure 3.14: 5th percentile throughput (a) in macrocell; (b) in picocell. With $\lambda=$ 4.6 marks $/ \mathrm{Km}^{2}$ and $\lambda^{\prime}=13.8$ marks $/ \mathrm{Km}^{2}$.

\subsubsection{Comparison with Real BS Deployment}

We obtained the data of real BS locations in United Kingdom from an organization [126] where the mobile network operators have voluntarily provided the information of location and operating characteristics of individual BSs. The data set in [126] was last updated in May 2012, and it provides exact locations of the BSs. Also, the BSs of different operators can be distinguished.

In this section, we compare the 5th percentile SE results from the PPP model with that of the real BS deployment and hexagonal grid model. The real MBS locations of two different operators in a $15 \times 15 \mathrm{~km}^{2}$ area of London city were obtained from [126] as shown in Figure 3.15. In this area, the average BS densities of the two operators were found to be $1.53 \mathrm{MBSs} / \mathrm{km}^{2}$ and $2.04 \mathrm{MBSs} / \mathrm{km}^{2}$. To have a fair comparison, the MBS locations for hexagonal grid and PPP models were also generated with the same densities. The PBS locations were generated randomly using another PPP model. The parameters $\tau=6 \mathrm{~dB}$, $\alpha=0.5, \beta=0.5, \rho=4 \mathrm{~dB}, \rho^{\prime}=12 \mathrm{~dB}$, and $P_{\mathrm{tx}}=46 \mathrm{dBm}$ were fixed while the PBS density $\lambda^{\prime}$ was varied to analyze its effect on the 5 th percentile SE.

The plots of 5th percentile SE versus PBS density are shown in Figure 3.16 for the two operators. The 5 th percentile SE of operator- 2 is better than that of operator- 1 since the former has higher MBS density. As expected, the 5th percentile SE improves with the 

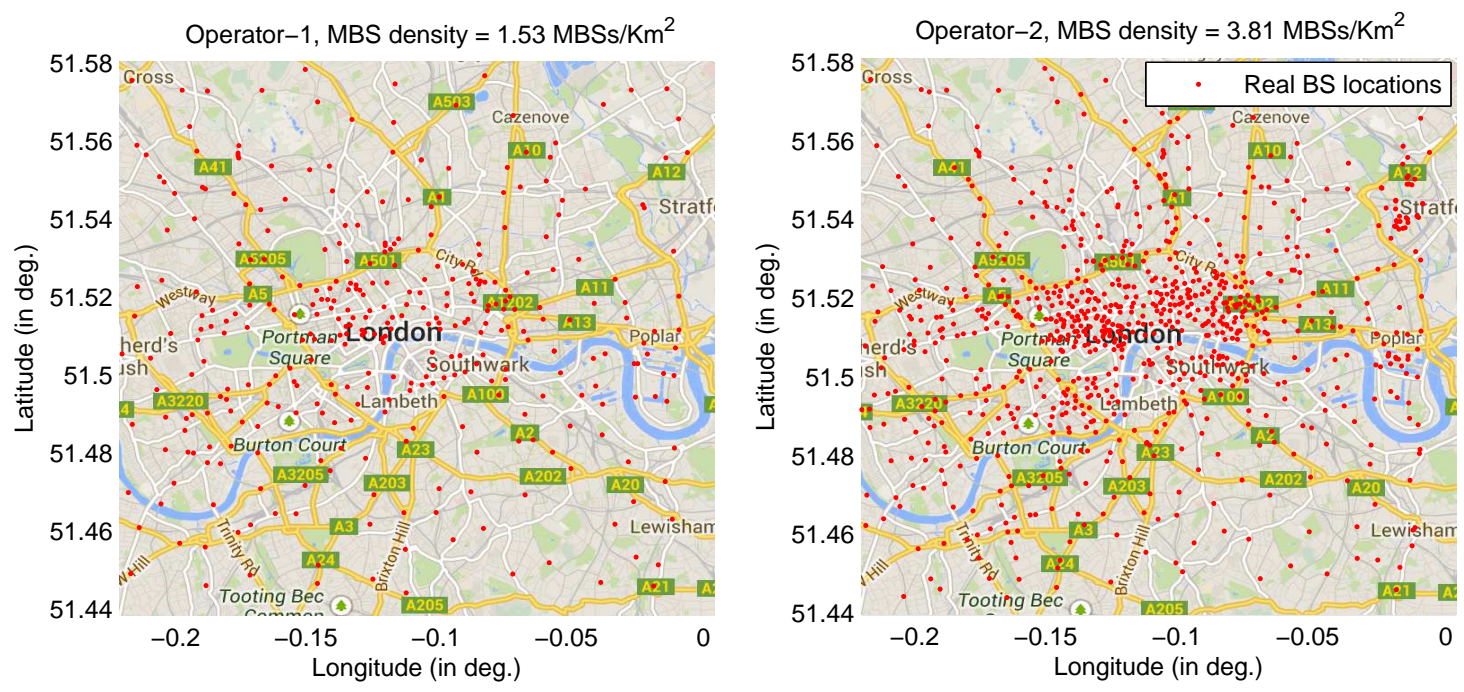

Figure 3.15: Real base station locations of two different operators in a $15 \times 15 \mathrm{~km}^{2}$ area of London city.

increase in PBS density. It can also be observed that increasing the PBS transmit power $P^{\prime}$ from $10 \mathrm{dBm}$ to $30 \mathrm{dBm}$ will result into almost twice the 5 th percentile SE. Since hexagonal grid model is an ideal case, it has the best 5th percentile SE and forms an upper bound. The PPP model has the worse 5th percentile SE and forms a lower bound. The real MBS deployment is usually planned and hence it is not completely random in nature. On the other hand, it is also not equivalent to the idealized hexagonal grid model due to the practical constraints involved during the deployment. Hence, the 5th percentile SE of real MBS deployment lies in between the hexagonal grid and random deployments.
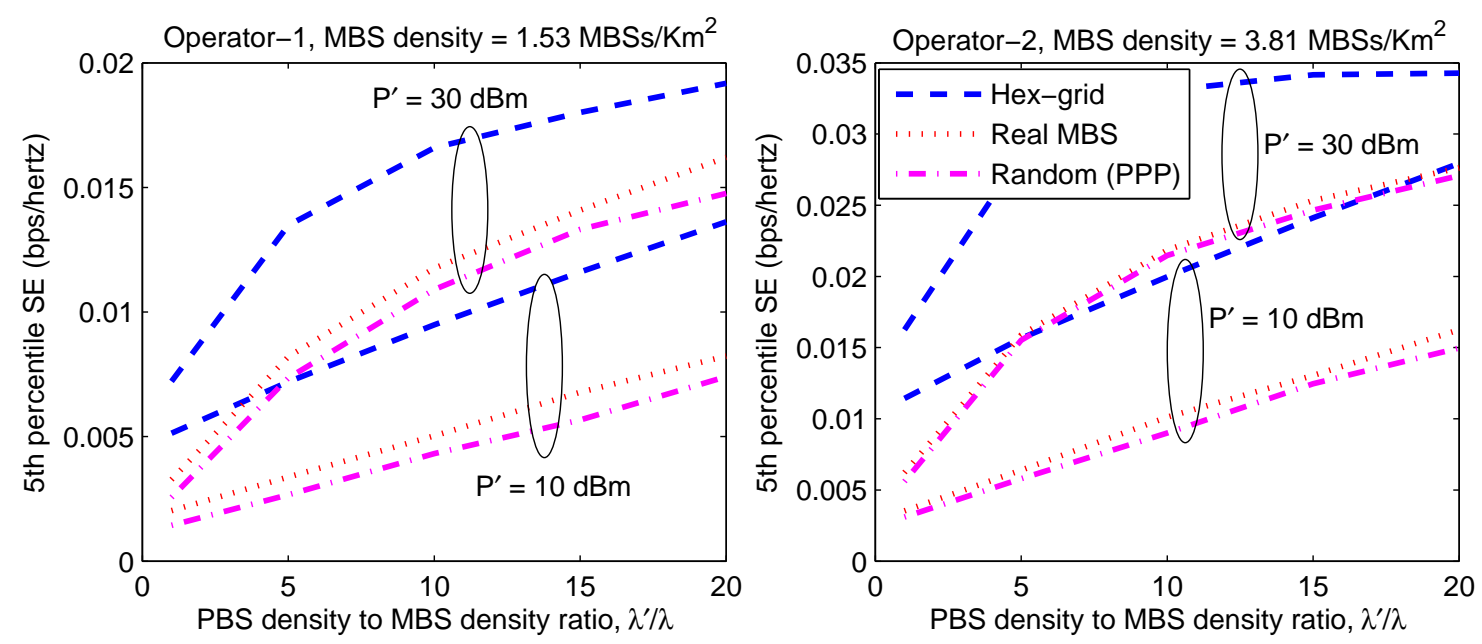

Figure 3.16: 5th percentile SE versus PBS density. 


\section{CHAPTER IV}

\section{Unmanned Aerial Base Stations in HetNets}

In recent years, UAVs are becoming popular in many applications, including wireless communications. An UAV or a group of UAVs equipped with small cell BSs can be deployed immediately in order to provide on-demand capacity enhancement in a particular geographical area of interest. This feature of UAVs is very helpful for PSCs during emergency situations where communications play a critical role in saving lives. Since the current communication technologies heavily rely on the backbone network, the failure of BSs due to natural disasters or malevolent attacks causes communication difficulties for public safety and emergency communications.

Lately, the use of UAVs such as quadcopters and unmanned gliders have gained attention in PSCs. They can be used as UABSs, which can be deployed rapidly as a part of the HetNet architecture. However, due to their mobile characteristics, interference management in the network becomes very challenging. In this chapter, we explore the use of UABSs for PSCs during natural disasters, where part of the communication infrastructure becomes damaged and dysfunctional (e.g., as in the aftermath of the 2011 earthquake and tsunami in Japan). Through simulations, we analyze the throughput gains that can be obtained by exploiting the mobility feature of the UAVs. Our simulation results show that when there is loss of network infrastructure, the deployment of UABSs at optimized locations can improve the SE coverage and the 5 th percentile SE of the network. This improvement is observed to be more significant with higher path-loss exponents. Furthermore, using REB in the UABS cells is shown to provide additional gains in the 5th percentile SE of the network.

\subsection{Public Safety Communications Scenarios}

This chapter aims to explore a new generation of broadband PSC systems that include UAVs for addressing the intricate interference management challenges in emerging PSC scenarios. In particular, we introduce a UAV assisted HetNet system that will constitute 


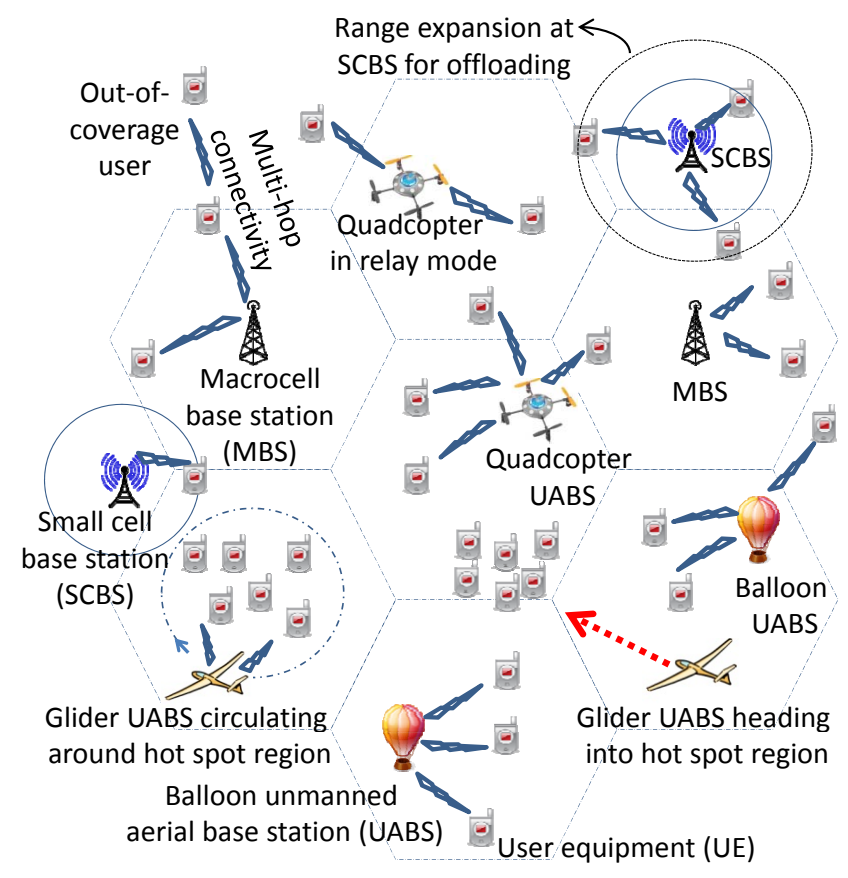

Figure 4.1: Large scale PSC scenario. The MBSs, SCBSs, and UABSs constitute a heterogeneous network (HetNet) infrastructure, where the UABSs can dynamically change their positions for optimized coverage and seamless broadband connectivity.

the pillar of the next generation of PSC systems. To provide a context for the proposed system, two representative PSC scenarios are discussed next.

\subsubsection{Scenario-1: Large-scale PSC scenario}

The first typical public safety scenario, illustrated in Figure 4.1, involves providing capable PSCs in diaster-affected environments following the aftermath of an earthquake, tsunami, or hurricane. In such environments, there is a vital need to maintain broadband, high-speed communication between first responders and victims, whose basic communication mediums may be jeopardized by damaged networking infrastructure as in the 2011 Earthquake in Japan [127]. In the representative scenario shown in Figure 4.1, only two of the seven MBSs with large coverage areas remain operational after a disaster. The figure also illustrates a number of SBSs, which can be critical to maintain connectivity in PSC scenarios. Range expansion techniques [22] are commonly used with the SBSs to extend coverage and fairly distribute users among different cells. To sustain ubiquitous broadband connectivity, 
Figure 4.1 shows how different types of UAVs are serving as UABSs. In hot-spot regions with denser UE population, quadcopters can hover at a fixed location, while gliders have to follow a circular trajectory. Relaying and multi-hop communication methods can also be used for extending the coverage through the incident scene, either through UAVs or other UEs.

\subsubsection{Scenario-2: Small-scale PSC scenario}

A second, typical PSC scenario involves a smaller-scale environment as in Figure 4.2, representative of scenarios such as a building on fire or a barricaded suspect. The incident scene is served by a number of SBSs, which are embedded into police cars, fire trucks, and UAVs. These SBSs provide broadband connectivity to first responders and victims in a timely manner, typically for deep situational awareness purposes through real-time wireless video streaming. Some example use cases of broadband connectivity include transmission of $3 \mathrm{D}$ blueprints of a burning building to the hand-held devices of first responders, live streaming of high definition video of the incident scene from the cap-mounted cameras (or Google-glass like gadgets) of the first responders to a command center, and transmission of multichannel vital signs of a hurt disaster victim to an external medical post to facilitate the diagnosis in collaboration with the on-site emergency medical staff and a remote specialist [128]. By exploiting UAV mobility, broadband connectivity can be delivered into desired regions, including congested areas and indoor environments.

A major challenge in the UAV assisted HetNets is to address the severe and highly dynamic interference challenges during/after the disaster scenarios as shown in Figure 4.1 and Figure 4.2. When compared with conventional heterogeneous network (HetNet) interference management techniques, scenarios such as in Figure 4.1 and Figure 4.2 are particularly unique and challenging due to 1) potentially damaged BS infrastructure, yielding outage problems; 2) mobility of SBSs in UAVs, police cars, and fire trucks, resulting in dynamic interference patterns and uncoordinated final locations of SBSs; 3) dynamically changing locations of UEs following a disaster, potentially clustered into some hot-spot areas; 4) HetNet traffic with bursty data transmission (e.g., post-earthquake) which may temporarily 


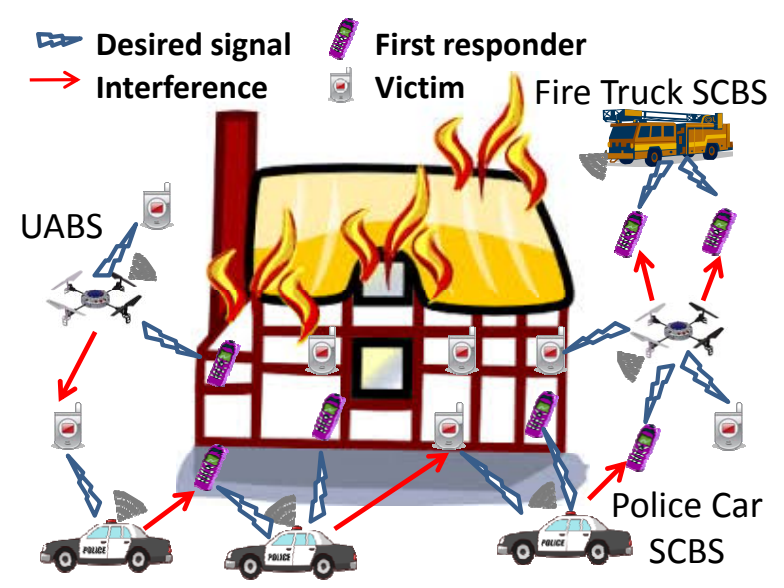

Figure 4.2: Building fire scenario.

overload the network infrastructure; and 5) the need to maintain high QoS for public safety personnel in mission-critical scenarios.

In this research, we focus on the large scale PSC scenario which is illustrated in Figure 4.1. We assume the MBS locations are randomly distributed according to a twodimensional homogeneous PPP, and perform Monte-Carlo simulations to evaluate SE coverage and 5th percentile SE of the network. We randomly remove some BS nodes to imitate a natural disaster, and then study the impact of infrastructure damage on the SE coverage and the 5th percentile SE. We also investigate the potential benefits of UAVs in the post disaster scenario by studying the capacity and coverage improvements achieved through the deployment of UAVs. Furthermore, we optimize the locations of the UAVs using $G A$ to maximize the 5th percentile SE of the network, and also study the effect of REB on the 5th percentile SE and the SE coverage.

\subsection{System Model}

We consider a cellular network with MBS and UE locations modeled as two-dimensional homogeneous PPPs of intensities $\lambda$ and $\lambda_{\mathrm{u}}$, respectively. PPP-based models may not be viable for capturing real $M B S$ locations, due to some points of the process being very close to each other. However, the PPP-based models provide reasonably close performance results 
when compared with the real BS deployments [22]. We assume all the MBSs transmit at power $P_{\mathrm{tx}}$, and every UE connects to its nearest MBS. For an arbitrary UE $n$ at a distance $r_{n m}$ from its serving MBS $m$, the average received signal power is given by

$$
S\left(r_{n m}\right)=\frac{P_{\mathrm{tx}} K}{r_{n m}^{\delta}}
$$

where $\delta$ is the path-loss exponent (PLE), and $K$ is the factor that accounts for the geometrical parameters such as transmitter and receiver antenna heights, etc. We consider an interference limited network in which the thermal noise power at a receiver is assumed to be negligible when compared to the interference power. Then, the SIR at UE $n$ can be expressed as,

$$
\Gamma_{n}=\frac{S\left(r_{n m}\right)}{\sum_{i \in \mathcal{M}, i \neq m} S\left(r_{n i}\right)},
$$

where $\mathcal{M}$ is the set of all MBSs, and $r_{n i}$ is the distance of $n$th UE to the $i$ th MBS. The denominator in (4.2) represents the total interference power at UE from all the MBSs except the serving MBS $m$. Using Shannon's capacity formula, and considering roundrobin scheduling for simplicity, the SE of a MUE can be expressed as,

$$
C_{n}=\frac{\log _{2}\left(1+\Gamma_{n}\right)}{N}
$$

where $N$ is the number of MUEs in the macrocell.

\subsubsection{SE Coverage}

Since achieving broadband rates is considered as a major goal, we use SE coverage as the performance indicator for network coverage ${ }^{1}$. a UE is considered to be in SE coverage if its average $\mathrm{SE}$ is higher than a threshold $T_{\mathrm{C}}$, i.e., $C>T_{\mathrm{C}}$. Here, we assume full buffer traffic in all the downlinks. The SE coverage in a typical network is illustrated in Figure 4.3a for

\footnotetext{
${ }^{1}$ Note that in LTE, outage (radio link failure) is normally declared when the wideband SINR is lower than a threshold. Since we envision network that serves all users at minimum guaranteed throughput, we consider a different coverage criteria for broadband communication applications.
} 
a $3 \times 3 \mathrm{~km}^{2}$ region in which the MBS intensity $\lambda=4$ per $\mathrm{km}^{2}$, and MBS transmit power $P_{\mathrm{tx}}($ in $\mathrm{dBm})=46 \mathrm{dBm}$. In Figure $4.3 \mathrm{a}$, the black lines indicate the cell boundaries, while the blue colored areas correspond to the SE coverage areas in which the UEs' SE are greater than the threshold $T_{\mathrm{C}}=3.5 \times 10^{-3} \mathrm{bps} / \mathrm{Hz}$, with a UE intensity of $\lambda_{\mathrm{u}}=400$ per $\mathrm{km}^{2}$. The white colored regions, on the other hand, are areas where the SE is lower than the threshold $T_{\mathrm{C}}$, which is negligible in Figure 4.3a.

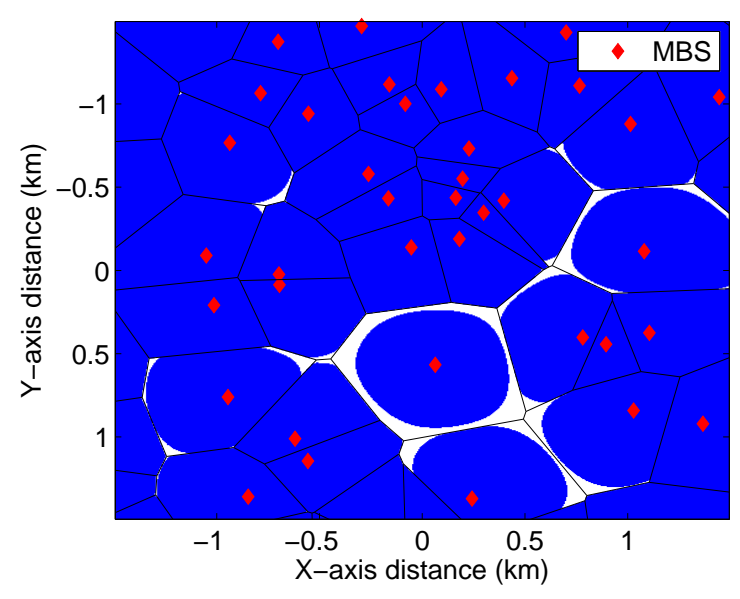

(a) Before earthquake

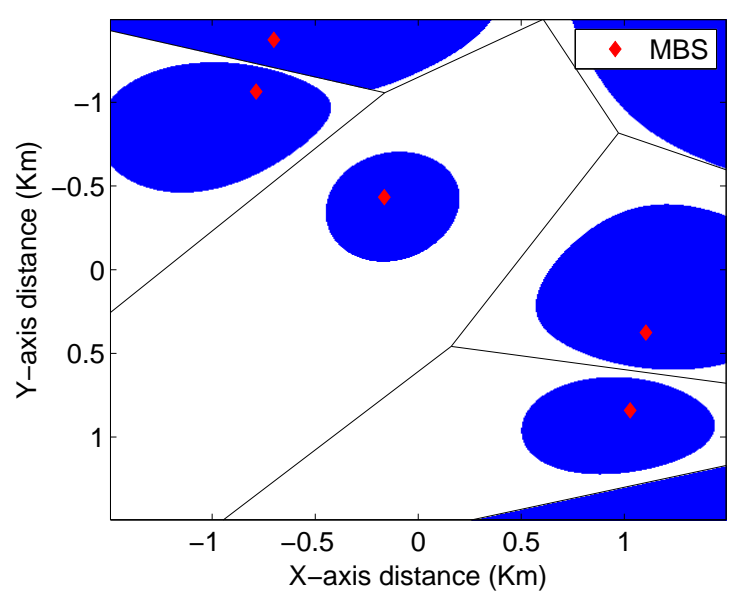

(b) After earthquake

Figure 4.3: Illustration of SE coverage in a typical network.

To illustrate the impact of infrastructure loss on coverage and SE, an example scenario of the cellular network after an earthquake is shown in Figure $4.3 \mathrm{~b}$, where $90 \%$ of the MBSs are randomly destroyed during a disaster, and the same $\mathrm{SE}$ threshold $T_{\mathrm{C}}$ as in Figure 4.3a is used to calculate outage regions. In this second scenario, outage region grows significantly, due to 1) overloading of MBSs with many UEs, and 2) poor link qualities of UEs far away from the MBSs. In a PSC scenario, first responders and victim users located within a white region in Figure 4.3b will observe very low SE, or even a complete outage and loss of connectivity. Naturally, for higher SE thresholds, the outage region will grow even larger.

\subsection{Coverage Improvement Using UABSs}

In the scenario of loss in network infrastructure as in Figure 4.3b, UABSs can be deployed rapidly to form small-cells and consequently improve the network coverage. Unlike MBSs, 
the UABSs' positions can be dynamically adjusted and therefore their physical locations can be optimized in order to get the best network performance for a given scenario.

With the deployment of UABSs, a HetNet is formed with two tiers of BSs: MBSs and UABSs. We assume that both the MBSs and the UABSs share a common transmission bandwidth. For simplicity, we assume that the wireless backhaul links of the UABSs have very large capacity, and they use a different frequency band than the access links. For an arbitrary UE $n$, let the nearest MBS $m$ at a distance $r_{n m}$ be its MBS of interest (MOI) and the nearest UABS $u$ at a distance $r_{n u}$ be its UABS of interest (UOI). Then, the average received signal power from the MOI and the UOI are respectively given by

$$
S\left(r_{n m}\right)=\frac{P_{\mathrm{tx}} K}{r_{n m}^{\delta}}, \quad S^{\prime}\left(r_{n u}\right)=\frac{P_{\mathrm{tx}}^{\prime} K^{\prime}}{r_{n u}^{\delta}}
$$

where $P_{\mathrm{tx}}^{\prime}$ is the transmit power of UABSs, and $K^{\prime}$ is the factor that accounts for the geometrical parameters such as the transmitter and receiver antenna heights. Then, an arbitrary UE experiences the SIRs,

$$
\begin{aligned}
\Gamma_{n} & =\frac{S\left(r_{n m}\right)}{\sum_{i \in \mathcal{M}, i \neq m} S\left(r_{n i}\right)+\sum_{j \in \mathcal{U}} S^{\prime}\left(r_{n j}\right)}, \\
\Gamma_{n}^{\prime} & =\frac{S^{\prime}\left(r_{n u}\right)}{\sum_{i \in \mathcal{M}} S\left(r_{n i}\right)+\sum_{j \in \mathcal{U}, j \neq u} S^{\prime}\left(r_{n j}\right)},
\end{aligned}
$$

from the MOI and the UOI, respectively. Here, $\mathcal{U}$ is the set of all UABSs, and $r_{n j}$ is the distance of $n$th UE to the $j$ th UABS. The denominators of (4.5) and (4.6) represent the total interference power at the UE. We assume that the UABSs employ range expansion bias (REB) during the UE association process in order to associate with more number of UEs. Each UE performs cell selection by using $\Gamma_{n}, \Gamma_{n}^{\prime}$ and the REB $\tau$ as follows:

$$
\begin{aligned}
& \text { If } \Gamma_{n}>\tau \Gamma_{n}^{\prime} \rightarrow \text { select MOI, } \\
& \text { If } \Gamma_{n} \leq \tau \Gamma_{n}^{\prime} \rightarrow \text { select UOI. }
\end{aligned}
$$


Table 4.1: System parameters

\begin{tabular}{|l|l|l|}
\hline Parameter & Description & Value \\
\hline$\lambda, \lambda_{\mathrm{u}}$ & MBS and UE intensities & 4 per $\mathrm{km}^{2}, 100 \mathrm{per}^{2}$ \\
\hline$P_{\mathrm{tx}}, P_{\mathrm{tx}}^{\prime}$ & MBS and UABS transmit powers & $46 \mathrm{dBm}, 30 \mathrm{dBm}$ \\
\hline$K, K^{\prime}$ & $\begin{array}{l}\text { Factors accounting for the geometrical parameters of an- } \\
\text { tennas }\end{array}$ & $-11 \mathrm{~dB},-11 \mathrm{~dB}$ \\
\hline$\delta$ & Path-loss exponent & 4 \\
\hline$\tau$ & Range expansion bias & $0 \mathrm{~dB}$ \\
\hline$d_{\mathrm{h}}$ & Altitude of UABSs & $121.92 \mathrm{~m}$ \\
\hline$T_{\mathrm{C}}$ & SE threshold & $2.55 \times 10^{-3} \mathrm{bps} / \mathrm{Hz}$ \\
\hline$A_{\mathrm{sim}}$ & Simulation area & $10 \times 10 \mathrm{~km}^{2}$ \\
\hline- & GA population size and generation number & 60 and 100 \\
\hline- & GA crossover and mutation probabilities & 0.7 and 0.1 \\
\hline
\end{tabular}

Finally, the SEs of MUE and UABS-cell UE (UUE) can be respectively expressed as,

$$
C_{n}=\frac{\log _{2}\left(1+\Gamma_{n}\right)}{N}, \quad C_{n}^{\prime}=\frac{\log _{2}\left(1+\Gamma_{n}^{\prime}\right)}{N^{\prime}},
$$

where $N^{\prime}$ is the number of UUEs in the UABS-cell.

\subsubsection{Optimization of UABSs' locations using Genetic Algorithm}

We use genetic algorithm (GA) to simultaneously optimize the locations of UABSs in a way to maximize 5th percentile SE of the network over a given geographical area. Here, each UABS is assumed to know the locations of other UABSs and MBSs. The GA proposed by Holland [129] is a global optimization technique based on genetic science. One of the advantages of GA is that it runs with population of candidate solutions rather than a single solution. Due to its parallel search capability, it can search over whole working environment simultaneously, so that an optimum solution can be obtained more quickly than the classical optimization techniques such as brute force search. Recently, GA has been used to solve deployment problem of wireless sensor networks, as well as optimization problems such as the traveling salesman problem.

With the approach of GA, a candidate solution for the optimization problem is referred as a chromosome, which is a set of all the UABSs' location coordinates, as illustrated in Figure 4.4. The optimization process begins with an initial population of chromosomes that are generated randomly, and runs for a certain number of iterations until the optimum solution is reached. In each iteration of the GA process, following steps are performed: 


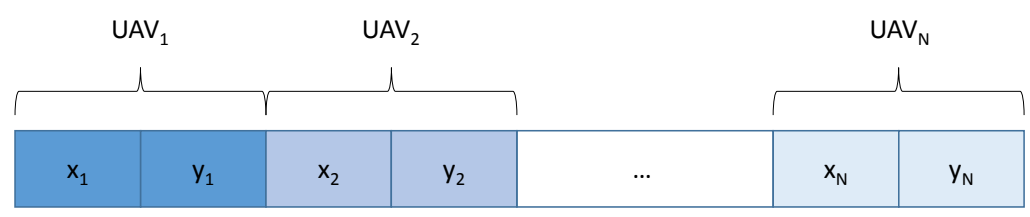

Figure 4.4: An example of a chromosome, where the parameters that need to be optimized are the individual locations $\left(x_{i}, y_{i}\right)$ of each UABS $i$, where $i=1,2, \ldots, N$.

1. All chromosomes are evaluated according to a fitness function. In our application, the 5th percentile SE of the network is the fitness function.

2. The selection process is used for determining the best chromosomes in the population which provide higher 5 th percentile SE results.

3. A Crossover process is used which combines the features of two parent chromosomes to generate two offspring.

4. After a new generation is created through crossover operator, new chromosomes are randomly chosen for mutation process. The mutation process is used in order to avoid the solution from converging to a local minima.

Figure 4.5 shows optimized placements of the UABSs using GA in a damaged network, in a way to maximize the 5th percentile SE over the whole network. In the simulations, about $90 \%$ of the MBSs were removed randomly in order to imitate loss of infrastructure. Each UABS is assumed to know the locations of other UABSs and BSs. In the considered architecture, it is assumed that the UABSs can communicate through a backhaul link to a nearby BS node $[130,131]$. The UABSs are observed to be clustered around the cell edges of macrocells in order to take over the low-SIR UEs and maximize the 5th percentile rate. Figure 4.5(a) shows an optimized placement of 5 UABSs at an altitude of $152.4 \mathrm{~m}$, while Figs. 4.5(b) and 4.5(c) show optimized placements of 10 UABSs at altitudes of $152.4 \mathrm{~m}$ and $45.72 \mathrm{~m}$, respectively. It can be observed that $10 \mathrm{UABSs}$ can achieve higher SE coverage area than 5 UABSs. Further, the SE coverage area increases approximately by $4 \%$ when the altitude of the UABSs decreases from $152.4 \mathrm{~m}$ to $45.72 \mathrm{~m}$. 


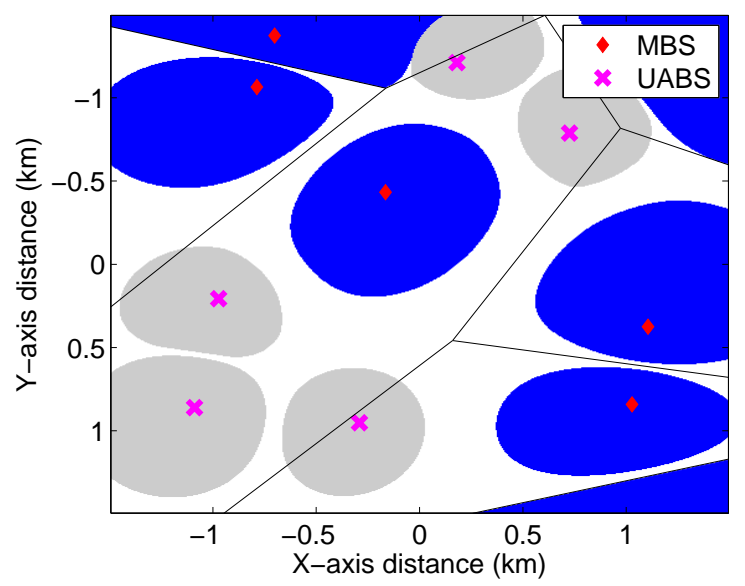

(a) 5 UABSs at $152.4 \mathrm{~m}$ altitude

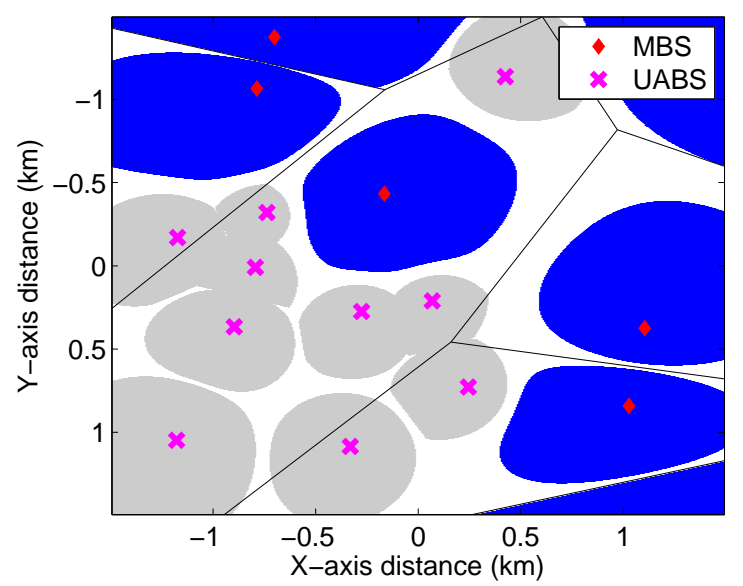

(b) 10 UABSs at $152.4 \mathrm{~m}$ altitude

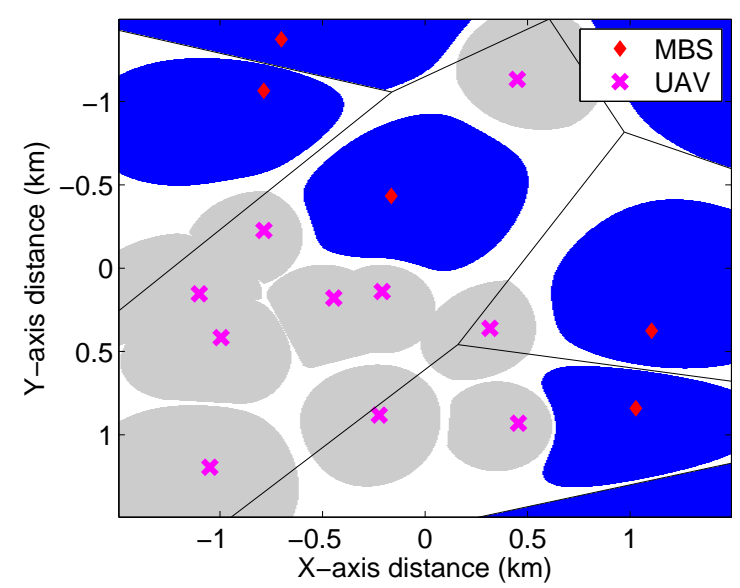

(c) 10 UABSs at $45.72 \mathrm{~m}$ altitude

Figure 4.5: Illustration of a damaged network with optimized placements of UABSs using genetic algorithm.

\subsubsection{Range Expansion for UABS Cells}

Due to the low transmit power of UABSs when compared to that of the MBSs, the UABSs might not be able to associate with more number of UEs. Using REB for small cells is one of the extensively studied techniques in HetNets to offload the macrocell UEs to the small cells for balancing the traffic load between the macrocells and small cells [22]. The range expansion has been included in LTE Release-8 specifications (standardized in 2008), and can also be used with all the future releases of LTE. In this technique, a UE in the network adds a REB to the received signal strength from the UABSs during the cell selection process, which increases the probability of selecting the UABSs for their serving cell. 


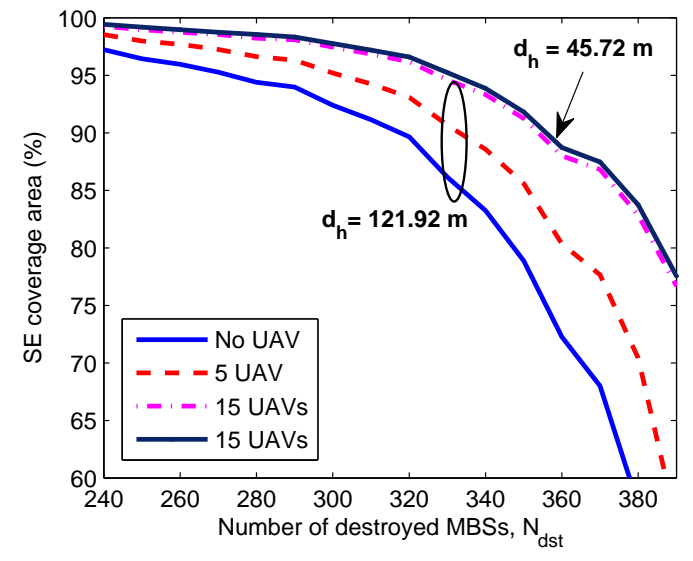

(a) SE coverage area versus $N_{\mathrm{dst}}$

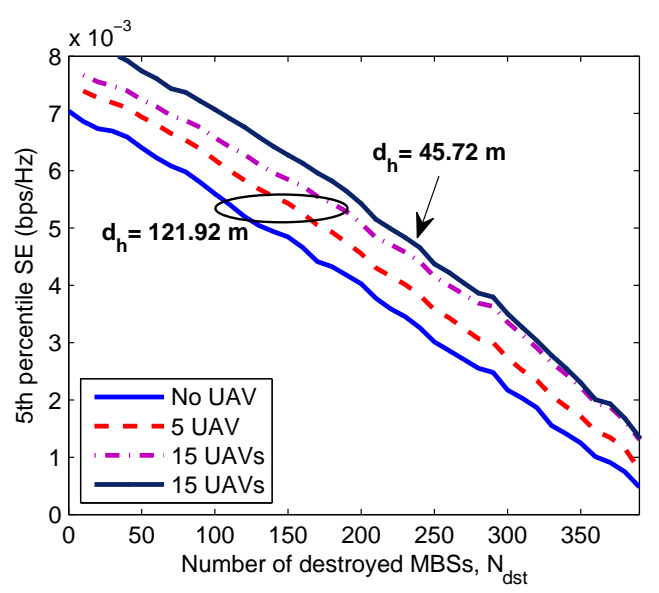

(b) 5 th percentile $\mathrm{SE}$ versus $N_{\mathrm{dst}}$

Figure 4.6: Improving SE coverage and 5th percentile SE with UABSs.

\subsection{Simulation Results}

The ability of UABSs to move to any location and height on an incident area provides a powerful mechanism to maintain high SE coverage throughout the network. In this section, we investigate the impact of optimized deployment and range expansion of UABS cells on the network performance. The simulation parameters were set to the values as shown in Table 4.1, and the locations of all UABSs were optimized through GA to maximize 5th percentile SE of the network. The 5th-percentile SE is a performance measure for cell edge users, and maximizing the 5th percentile SE will ensure that cell edge users continue to maintain good spectral efficiency.

\subsubsection{Impact of Number of Destroyed MBSs}

The SE coverage area and the 5th percentile SE as a function of the number of destroyed MBSs $N_{\text {dst }}$, (out of a total 400) are shown in Figure 4.6, with different number of helper UABSs. The SE coverage area is defined as the percentage of area with SE larger than

a threshold $T_{\mathrm{C}}$ (taken as $2.55 \times 10^{-3} \mathrm{bps} / \mathrm{Hz}$ in simulations), versus the whole simulation area. These results show that the height $\left(d_{\mathrm{h}}\right)$ of a UABS has relatively limited effect on SE coverage, but has a more pronounced effect on the 5th percentile SE due to path loss factors. Figure 4.6 also shows that 15 optimally-positioned UABSs can handle the load of 


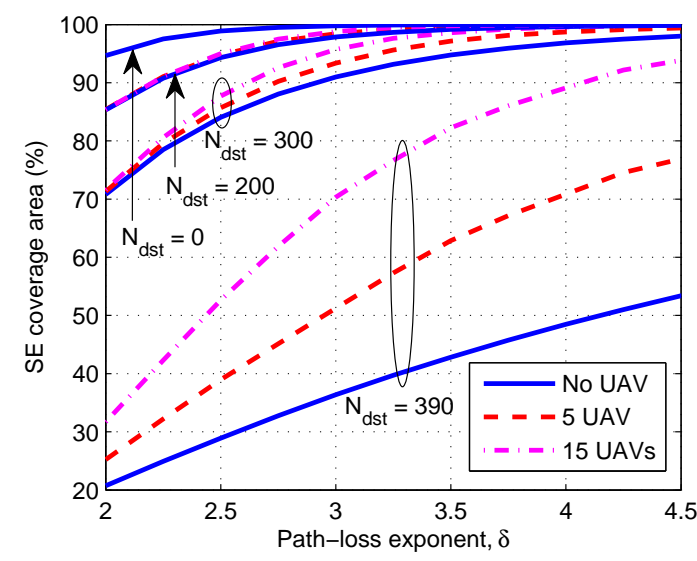

(a) SE coverage area versus PLE

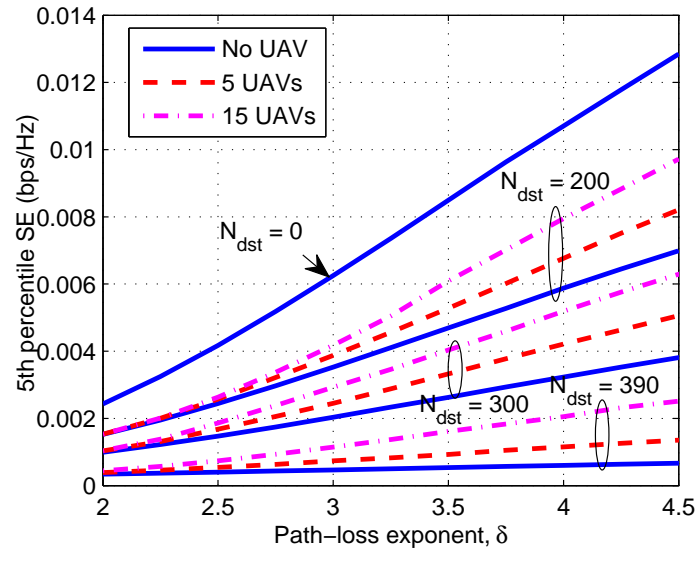

(b) 5th percentile SE versus PLE

Figure 4.7: Effects of PLE on SE coverage area and 5th percentile SE.

up to $70 \mathrm{MBSs}$ from a SE coverage area perspective, while the gains are lower when the 5th percentile SE is considered. Lowering the UABS height (assuming line-of-sight scenarios) is also shown to bring additional SE benefits due to lower path loss.

\subsubsection{Impact of Path-loss Exponent}

The characteristics of SE coverage and 5 th percentile SE with respect to variations in the PLE are shown in Figure 4.7. In general, both the SE coverage and the 5th percentile SE improve with the increasing PLE because the interference power at a UE decreases more rapidly than the signal power as the PLE increases, thereby improving the SIR at the UE. This is due to the fact that the UE's distance to its connected BS is lesser than all other interfering BSs. Figure 4.7 also shows that the SE coverage improves with more number of UABSs, and the improvement is significant with higher PLE. Particularly, the case when 390 out of 400 MBSs are destroyed, approximately $94 \%$ of the area can still be covered with just 15 UABSs, provided the PLE is 4.5 .

\subsubsection{Impact of Range Expansion Bias}

Figure 4.8 shows the variations of SE coverage probability, 5th percentile SE, and the CDF of per-user SE, with respect to the REB. It can be observed in Figure 4.8(a) that the 


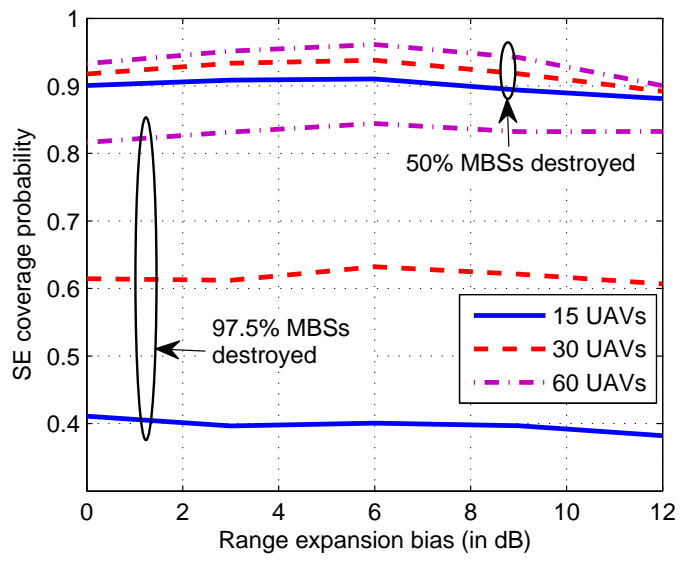

(a) SE coverage probability versus REB

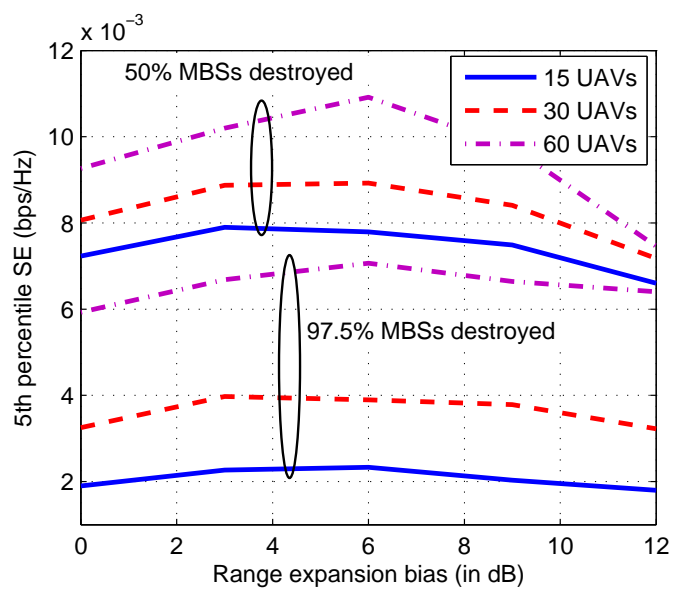

(b) 5th percentile $\mathrm{SE}$ versus REB

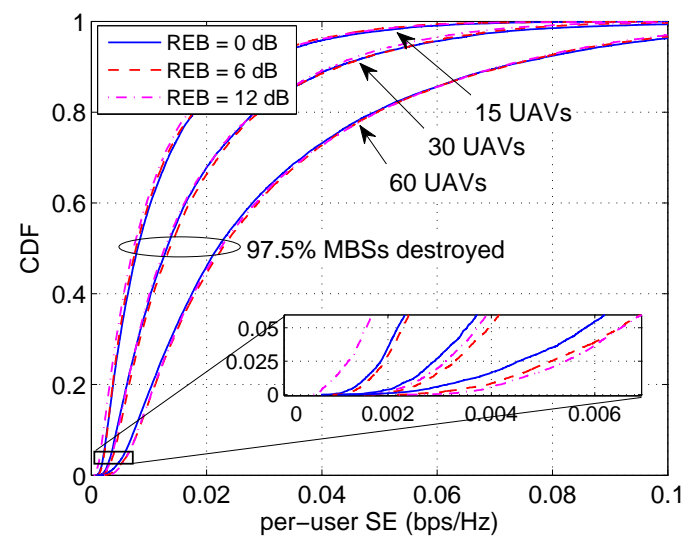

(c) $\mathrm{CDF}$ of the per-user SE versus REB

Figure 4.8: Effects of REB on SE coverage probability and 5th percentile SE.

SE coverage probability is maximized at a particular REB value; however, the coverage probability gain at this optimum REB is limited. On the other hand, the effect of REB on the 5th percentile SE is notable as shown in Figure 4.8(b). For example, when $50 \%$ of MBSs are destroyed in $10 \times 10 \mathrm{~km}^{2}$ area and $60 \mathrm{UAVs}$ are deployed at optimal locations, using an REB of $6 \mathrm{~dB}$ increases the 5 th percentile $\mathrm{SE}$ by $18 \%$ when compared to an REB of 0 dB. It can also be noted in Figs. 4.8(a) and 4.8(b) that the effect of REB on SE coverage probability and 5th percentile SE is more significant when higher number of UAVs are deployed.

The effects of different number of destroyed MBSs are also shown in Figs. 4.8(a) and 4.8(b). With the increasing number of destroyed MBSs, the SE coverage probability and the 5th percentile SE both decrease due to the decreasing density of MBSs. In such a scenario, 
the SE coverage probability and the 5th percentile SE can be increased significantly by deploying more number of UABSs at optimized locations. For example, assuming that 97.5\% of MBSs are destroyed, by deploying 60 UAVs at optimized locations and using an REB of $6 \mathrm{~dB}$, the 5 th percentile SE can be improved by $\sim 203 \%$ and the SE coverage probability can be improved by $\sim 106 \%$ when compared to the deployment of 15 UAV. Figure 4.8(c) shows the CDF of the per-user SE for different number of deployed UAVs and for different REB values. With more number of deployed UAVs, the CDF shifts towards right side of the graph indicating better SEs for the users. 


\section{CHAPTER V \\ Capacity Enhancement through Subsidy Regulations}

\section{$5.1 \quad$ Introduction}

Traditional regulatory methods for spectrum licensing have been recently identified as one of the causes for the under-utilization of the valuable radio spectrum. Governmental regulatory agencies such as the FCC are seeking ways to remove stringent regulatory barriers and facilitate broader access to the spectrum resources. The goal of such new FCC-backed efforts is to allow for an improved and ubiquitous sharing of the precious radio spectrum between commercial service providers.

In this chapter, a novel noncooperative game is proposed to analyze how to foster more sharing of the radio spectrum via the use of regulatory power. A two stage game is defined in which the government regulators move first, followed by the providers. The providers are incentivized by lower spectrum allocation fees from the regulators in return for proofof-sharing. The providers are offered discounted spectrum bands, potentially at different locations, but will be asked to provide coverage to users that are not subscribed to them so as to maintain their subsidy incentives from the government.

Roaming agreements and spectrum sharing among the traditional operators are alternative approaches to the proposed subsidy schemes. However, roaming agreements are not common among different types of network operators, such as among cellular operators and public safety operators. While roaming agreements can make spectrum sharing possible among the service providers, it penalizes the customers and the small service providers with roaming charges, and may result in monopolization. On the other hand, the proposed subsidy scheme is an alternative solution which is seamless to the customers and can enhance spectrum sharing among the service providers through the incentives from government, while avoiding monopolization. 


\subsubsection{Motivations}

The benefits of subsidizing the spectrum to the providers can be described as follows:

- Participation of the providers and coordination between them is important for the greater good applications in wireless communications. Example applications include the public safety communication services offered by the commercial service providers. In summary, subsidizing the spectrum to the providers helps in improving the quality of service to the users through the providers' participation.

- Since the spectrum resources are limited, spectrum sharing is important in order to use the precious spectrum resources efficiently. Subsidization helps to improve the spectrum sharing among the providers since the government asks the providers to provide service to the foreign customers.

- The process of subsidizing the spectrum to the providers will ultimately improve users' experience. Furthermore, the entire process of the subsidization will be seamless to the users.

- Finally, the government can prevent monopolization in the market by allocating the subsidy appropriately to the different providers. This helps the small providers to effectively compete with the existing large providers.

\subsubsection{Contributions}

In this chapter, we model a subsidization framework using an extensive form game, and show how to find (perfect) equilibrium solutions for (a simple case of) the game. The players ${ }^{1}$ in the game are a finite number of service providers plus a single government player. In the legacy cellular market, providers do not have any incentive to serve foreign customers, i.e., subscribed to another provider. Customers are effectively forced to their home providers (i.e., the provider they are subscribed to), even if there may be another provider with a better signal for their location.

\footnotetext{
${ }^{1}$ Customers are not formal players in our game as their actions are completely determined by the moves of the providers and the government.
} 
In our proposed model, the government incentivizes by offering subsidy to the providers so that they give service to foreign customers who are outside the coverage of their home provider or simply in a spot where their home provider's signal quality is worse than other providers they are not subscribed to. Yet, the government also expects the providers to share their spectrum and infrastructure resources with foreign calls. If a provider cannot satisfy a proof-of-sharing level, it has to return some or all of the subsidy back to the government. Thus, the providers each aim to maximize their profit from enrolling customers, by efficiently investing the subsidies received from the government. A customer's utility depends upon the level of service obtained from its home provider as well as the service it receives from foreign providers.

The government's most important goal in our spectrum subsidization model is to increase the quality of experience the users receive, and thus, to maximize social welfare, here measured as the sum of the customers' utilities. In the subsidization framework, we do not consider the implementation details of collecting the information of the number of foreign calls served by a provider and accounting of proof-of-sharing in the legacy cellular systems. We leave this topic for future research.

Initially, with preliminary observations of the game theoretic framework, we provide insights into the effects of subsidization on the service providers and the customers. However, due to the complexity of the problem, we cannot obtain the equilibrium solutions. For this reason, we consider a simple, yet insightful case of two providers and two regions. Here, we find the equilibrium solution through numerical computation of the equilibrium conditions. Since analytical expressions to the equilibrium solutions could not be derived, the existence of Nash equilibrium could not be proved analytically. However, we argue that our analysis implies this existence at least for some particular parameter values. We then present numerical results of the equilibrium solutions by using real $B S$ locations of two providers in two cities of the United Kingdom (UK). Through these results, we show that the government can significantly improve the average spectral efficiency of the users by providing subsidy to the providers. We further show that the government can avoid monopolization by a single provider by allocating higher subsidy to the other small scale providers. 


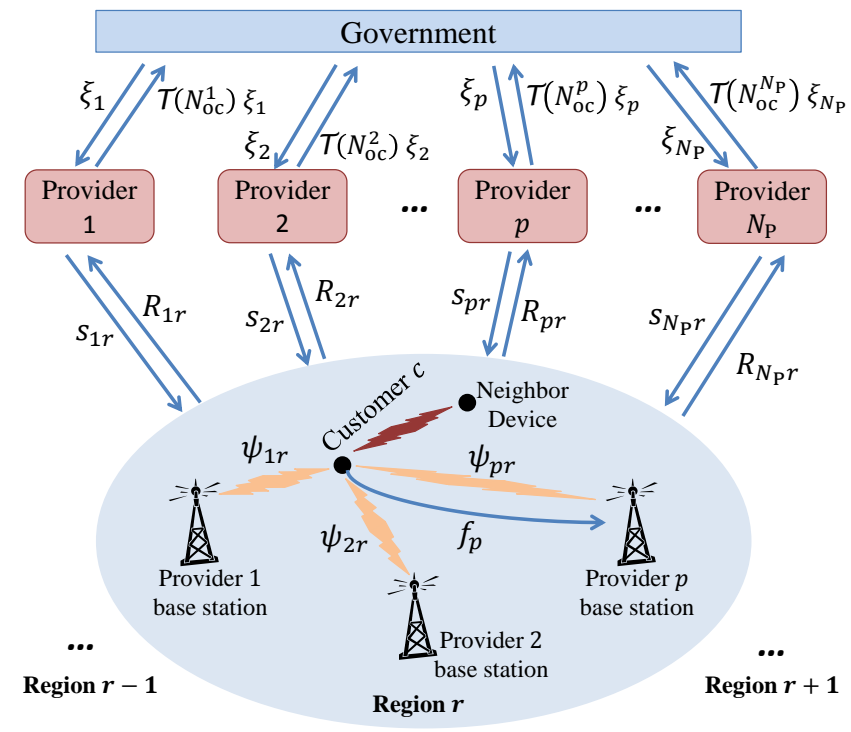

Figure 5.1: Model for a subsidized spectrum market. In this model, government allocates subsidy $\xi_{p}$ to provider $p$, which in return can utilize a portion $s_{p r}$ of it to improve the service quality (which is captured by $\psi_{p r}$ ) in region $r$. Provider $p$ charges fee $f_{p}$ to each of its customers and as a result collects a revenue $R_{p r}$ from region $r$.

\subsection{A Spectrum Market with Subsidy}

Even though there are several works in the literature on the role of government in market formations $[132,133]$, the government's regulatory role in the radio spectrum market has not been explored well within a game-theoretic framework. Here, we model the government's use of subsidies as a way to motivate the service providers to offer better service to the users in roaming, which the providers would not be likely to do on their own.

We propose a spectrum market model with three types of agents as shown in Figure 5.1: customers, providers, and a single government player. A list of all the notations used in our model appears in Table 5.1. The customers are essentially the end-user devices that will ultimately engage in localized spectrum sharing markets. Let $\mathcal{C}=\left\{1, \ldots, N_{\mathrm{C}}\right\}$ be the set of wireless customers. These customers are spread out over a set of regions $\mathcal{R}=\left\{1, \ldots, N_{\mathrm{R}}\right\}$.

We assume there are $n_{r}$ customers in region $r \in \mathcal{R}$. If $r_{c}$ is the region in which customer $c \in \mathcal{C}$ is located, we call $r_{c}$ his home region. Each customer $c$ makes $\beta_{c}$ calls in his home region (over the time period associated with the game); for simplicity we assume $\beta_{c}$ is equal 
to a constant $\beta$ for all $c$. In addition, we assume customers each make $\alpha$ calls outside their home region. To choose its main service provider, customer $c$ takes as given the intensity of service/signal $\psi_{p r_{c}}$ offered by each provider $p$ in region $r_{c}$, as well as the fixed fee $f_{p}$ that each $p$ charges for service over the service period. This is done probabilistically as described below.

The set of all providers is denoted by $\mathcal{P}=\left\{1, \ldots, N_{\mathrm{P}}\right\}$. Providers operate in all regions. The government gives each provider an allotment of bandwidth and of monetary subsidy, which they, in turn, allocate for their own use in each of the $N_{\mathrm{R}}$ regions. The amount of bandwidth and money spent in each region determines the intensity of service offered, which in turn helps determine who wins customer subscriptions. An important feature of this model is that the government motivates the providers to give service to customers who are outside of their home region (i.e., roaming) or simply far away from the base stations of their home providers. For such customers, foreign providers might be able to provide a higher intensity signal than the customers' home providers. The government aims to motivate providers by reducing their subsidies if they do not serve enough customers who are away from their home regions.

We propose a two-stage, extensive form noncooperative game [134] in which the government moves first, followed by the providers (all simultaneously). Mathematically, we would like to solve for a perfect Nash equilibrium in the game as follows: a) Knowing what the customers will do as a function of $\left\{\psi_{p r_{c}}\right\}_{c, p}$ and $\left\{f_{p}\right\}_{p=1}^{N_{\mathrm{P}}}$, we can solve for the optimal strategy for the providers; b) Knowing how the providers/customers behave, we can solve the governments problem, which would be to maximize social welfare - which in this case would be the total of customers' utility from consuming the wireless spectrum.

In the rest of this section, first, the utility that a customer $c$ can obtain by choosing a provider $p \in\left\{1,2, \ldots, N_{p}\right\}$ will be formulated in Section 5.2.1. Subsequently, in Section 5.2.2, provider $p$ 's problem will be formulated in which the objective is to maximize the profit of provider $p$. Section 5.2.3 defines the objective function for the government, which aims to maximize the social welfare by maximizing the customers' total utility. Finally, some key observations and discussions are presented in Section 5.2.4. 
Table 5.1: Notations and Symbols

\begin{tabular}{|l|l|}
\hline Symbol & Description \\
\hline \hline$N_{\mathrm{C}}$ & Total number of customers \\
\hline$N_{\mathrm{P}}$ & Total number of providers \\
\hline$N_{\mathrm{R}}$ & Total number of regions \\
\hline$n_{r}$ & Number of customers in region $r$ \\
\hline$\beta$ & Number of home calls made by each customer \\
\hline$\alpha$ & Number of outside calls made by each customer \\
\hline$r_{c}$ & The region where customer $c$ is located \\
\hline$f_{p}$ & The fee charged by provider $p$ to its customers \\
\hline$\psi_{p r}$ & The intensity/quality of provider $p$ 's signal in region $r$ \\
\hline$Q()$. & $\begin{array}{l}\text { An increasing concave function used to calculate } \psi_{p r}-\text { parameterized with } \\
\text { the investment, } s_{p r}, \text { and/or bandwidth, } b_{p r}, \text { in a region }\end{array}$ \\
\hline$u()$. & $\begin{array}{l}\text { Customers' utility function }- \text { parameterized with received signal intensity } \\
\psi_{p r}\end{array}$ \\
\hline$U_{\mathrm{c}}(p, r)$ & The utility obtained by choosing provider $p$ in region $r$ \\
\hline $\mathbb{P}_{p}(r)$ & The probability that provider $p$ is chosen in region $r$ \\
\hline$E_{p}$ & Cash on hand for provider $p$, before obtaining any subsidy \\
\hline$e_{p r}$ & A portion of $E_{p}$ that provider $p$ invests in region $r$ \\
\hline$\xi_{p}$ & Subsidy amount provider $p$ receives from the government \\
\hline$\xi$ & Total subsidy budget of the government \\
\hline$R_{p r}$ & Provider $p$ 's revenue collected from region $r$ \\
\hline$s_{p r}$ & $\begin{array}{l}\text { A portion of the investment amount } E_{p}+\xi_{p} \text { provider } p \text { designates/spends } \\
\text { in region } r\end{array}$ \\
\hline$N_{\mathrm{oc}}^{p}$ & Number of outside calls provider $p$ serves \\
\hline $\mathcal{T}(\cdot)$ & $\begin{array}{l}\text { Penalty function determining the proportion of the subsidy to be returned } \\
\text { to the government }- \text { parameterized with } N_{\mathrm{oc}}^{p}\end{array}$ \\
\hline$\delta$ & Per-outside-call reward to the provider from the government \\
\hline$\gamma$ & Scaling factor in the customer utility function \\
\hline
\end{tabular}

\subsubsection{Customer c's Problem}

We formulate the customer's problem based on the value for money that a customer obtains from a provider. Each customer $c$ has the same increasing concave utility function $u$ for intensity of wireless service, expressed in units of money per call. Thus

$$
U_{\mathrm{c}}\left(p, r_{c}\right)=\beta u\left(\psi_{p r_{c}}\right)-f_{p}
$$

measures the utility a customer would get from local calls in its home region $r_{c}$, if the customer chose provider $p$ as its home provider. The customer then chooses provider $p$ 
$\left(p=1, \ldots, N_{\mathrm{P}}\right)$ with probability

$$
\mathbb{P}_{p}\left(r_{c}\right)=\frac{U_{\mathrm{c}}\left(p, r_{c}\right)}{\sum_{p^{\prime}=1}^{N_{\mathrm{P}}} U_{\mathrm{c}}\left(p^{\prime}, r_{c}\right)}
$$

This probabilistic assignment of contracts (prizes) to providers (contestants) based on resources offered is taken from Contest Theory, and arises in many applications (see, e.g., $[135,136]$, or the excellent survey paper of [137]). Thus, we emphasize that customers do not necessarily choose the provider who offers the highest $U_{\mathrm{c}}\left(p, r_{c}\right)$; such providers are only more likely to be chosen. The reason for this is that while $U_{\mathrm{c}}\left(p, r_{c}\right)$ captures two important variables in what determines a customer's choice of service (the providers' fee structure and intensity of service), it doesn't capture other things: customers' lack of knowledge of all potential carriers and what they offer, their lack of rationality, their brand name loyalty, etc. Thus, while we would expect providers offering better fees/intensity to attract more customers, we would not expect them to attract all customers.

We note that customers choose the providers based only upon offered service in their home regions, because their outside calls will always be covered (at no extra cost to them) as a result of the government's subsidization scheme.

\subsubsection{Provider p's Problem}

The goal of the providers is to maximize their own profits by optimizing their investments into the different regions by considering the factors such as the number of customers in each region and their calling patterns, the amount of fee charged to the customers, and the competition from the other providers, etc. Provider $p$ has at its disposal a total amount of cash $E_{p}+\xi_{p}$, where $E_{p}$ is the amount of cash on hand at the beginning of the game, and $\xi_{p}$ is the amount of subsidy it receives from the government. They also are allocated bandwidth $B_{p}$ from the government. Their problem is to allocate these resources among the regions, designating $s_{p r}+e_{p r}$ to spend in region $r$ and bandwidth $b_{p r}$ to use in region 
$r, r=1, \ldots, N_{\mathrm{R}}$. The intensity of service that it offers in region $r$ is then

$$
\psi_{p r}=Q\left(s_{p r}+e_{p r}, b_{p r}\right)
$$

where $Q$ is an exogenously given function (increasing and concave). In other words, the service intensity monotonically increases with the cash invested by the provider, as well as the utilized bandwidth. The average spectral efficiency that a user experiences from provider $p$ in region $r$ can be used as a measure for the service intensity $\psi_{p r}$, which will be shown in Section 5.5.2. Provider $p$ also sets the fee $f_{p}$ which all of its contracted customers in all regions must pay.

From the description of the customers' problem above, we see that provider $p$ will capture $n_{r} \mathbb{P}_{p}(r)$ of the customers in region $r$, for a revenue of

$$
R_{p r}=f_{p} n_{r} \mathbb{P}_{p}(r)
$$

where $\mathbb{P}_{p}(r)$ is defined in (2). Then their total revenue (across all regions) is $\sum_{r=1}^{N_{\mathrm{R}}} R_{p r}$.

Next, we note that the customers from region $\hat{r}$ make $\alpha n_{\hat{r}}$ calls outside of their home region. We assume

$$
n_{\hat{r} r}=\frac{n_{r}}{\sum_{r^{\prime} \neq \hat{r}} n_{r^{\prime}}} \alpha n_{\hat{r}}
$$

of these occur in region $r(r \neq \hat{r})$. Thus the foreign calls that customers make outside their home region are allocated across regions proportionally according to the populations of those regions.

The $n_{\hat{r} r}$ calls from (5) are allocated probabilistically to the providers, according to the intensity levels offered by the providers in region $r$. Hence $n_{\hat{r} r} \frac{u\left(\psi_{p r}\right)}{\sum_{p^{\prime}=1}^{N_{\mathrm{P}}} u\left(\psi_{p^{\prime} r}\right)}$ of those calls are served by provider $p$. However, in order to be truly considered a outside call for provider $p$, we must take into account that $\mathbb{P}_{p}(\hat{r})$ of these calls (see (2)) are actually $p$ 's customers already, from region $\hat{r}$. We should not count these in the calculation of the number of foreign calls for $p$, so the true number of outside calls for provider $p$ made by customers from region $\hat{r}$ traveling in region $r$ is $n_{\hat{r} r} \frac{u\left(\psi_{p r}\right)}{\sum_{p^{\prime}=1}^{N_{\mathrm{P}}} u\left(\psi_{p^{\prime} r}\right)}$ multiplied by $1-\mathbb{P}_{p}(\hat{r})$. This in turns 
means that the total number of outside calls that provider $p$ serves is then

$$
N_{\mathrm{oc}}^{p}=\sum_{r=1}^{N_{\mathrm{R}}} \sum_{\hat{r} \neq r} n_{\hat{r} r} \frac{u\left(\psi_{p r}\right)}{\sum_{p^{\prime}=1}^{N_{\mathrm{P}}} u\left(\psi_{p^{\prime} r}\right)}\left(1-\mathbb{P}_{p}(\hat{r})\right) .
$$

The provider is then assessed a penalty according to this number - the lower the quantity $N_{\mathrm{oc}}^{p}$ is, the higher the penalty. The penalty is expressed as a proportion of subsidy lost, and is incurred after the market. So, assuming this penalty function is denoted $\mathcal{T}(\cdot)$, we can write down an optimization problem for provider $p$ :

$$
\begin{aligned}
& \max _{\left\{s_{p r}\right\},\left\{b_{p r}\right\}, f_{p}} \sum_{r=1}^{N_{\mathrm{R}}} R_{p r}+\left(1-\mathcal{T}\left(N_{\mathrm{oc}}^{p}\right)\right) \xi_{p}-\sum_{r=1}^{N_{\mathrm{R}}}\left(s_{p r}+e_{p r}\right) \\
& \text { such that, } \quad \sum_{r=1}^{N_{\mathrm{R}}} s_{p r} \leq \xi_{p}, \\
& \sum_{r=1}^{N_{\mathrm{R}}} b_{p r}=B_{p}, \\
& \psi_{p r}=Q\left(s_{p r}+e_{p r}, b_{p r}\right), \\
& s_{p r}, b_{p r}, f_{p} \geq 0, \forall p, r,
\end{aligned}
$$

where the first term in the maximization is the total revenue, the second term is the leftover subsidy money after the penalty is deducted due to fewer-than-required sharing of the provider's spectrum, and the last term is the total amount of expenses the provider uses from the subsidy it received from the government.

\subsubsection{Government's Problem}

The goal of the government is to maximize social welfare, here defined as the customers' total utility from wireless service. The government's decision variables are $\xi_{p}$ and $B_{p}$, $p=1, \ldots, N_{\mathrm{P}}$. It also can designate the function $\mathcal{T}(\cdot)$ described above. The objective 
function of the government can expressed as

$$
\begin{aligned}
\max _{\left\{\xi_{p}, B_{p}\right\}_{p=1}^{N_{\mathrm{P}}}} & {\left[\sum_{c=1}^{N_{\mathrm{C}}} \beta u\left(\psi_{p(c) r_{c}}\right)+\sum_{p=1}^{N_{\mathrm{P}}} \sum_{r=1}^{N_{\mathrm{R}}}\left(\sum_{\hat{r} \neq r} \frac{u\left(\psi_{p r}\right)\left(1-\mathbb{P}_{p}(\hat{r})\right)}{\sum_{p^{\prime}=1}^{N_{\mathrm{P}}} u\left(\psi_{p^{\prime} r}\right)} n_{\hat{r} r} u\left(\psi_{p r}\right)\right)\right] } \\
\text { s.t., } \quad & \sum_{p=1}^{N_{\mathrm{P}}} B_{p}=B, \\
& \sum_{p=1}^{N_{\mathrm{P}}} \xi_{p} \leq \xi \\
& B_{p}, \xi_{p} \geq 0 \forall p .
\end{aligned}
$$

Here, the first term in the objective function is the utility that customers acquire in their home regions, while the second term is from calls in outside regions. The notation $p(c)$ is the home provider to which customer $c$ is assigned. The quantities $B$ and $\xi$ represent the total amounts of bandwidth and subsidy that the government has at its disposal to allocate.

Note: The objective function could include a term negatively correlated to $\sum_{p=1}^{N_{\mathrm{P}}} \xi_{p}$, if one wishes to argue that government spending is a negative. We do not include such a term in our model.

\subsubsection{Observations and Discussion}

The model we have just outlined provides a good framework to consider the viability of subsidizing spectrum to providers in return for increased spectrum sharing. The providers constitute a key player to scrutinize in such a spectrum market model. Ultimately, the goal of this market model is to motivate the providers to share their spectrum among each other to attain the larger good of serving more customers with better quality access links.

Provider $p$ 's problem (5.7) offers several insights. The first term in (5.7) are the fees provider $p$ collects from its home customers, who are subscribed to provider $p$. Provider $p$ has to serve these customers by default. But when these customers travel to other regions, they may be able to find another provider that offers a better quality signal at that location. In that case, those customers would be considered as making an outside call, and thus contributing to the sharing of the spectrum among the providers. 
The second term in (5.7) describes the amount of subsidy money left to provider $p$. This leftover amount is dependent on the subsidy amount, $\xi_{p}$, given by the government to provider $p$, and more importantly, the number of outside calls, $N_{\mathrm{oc}}^{p}$, served by provider $p$ during the subsidy term. If $N_{\mathrm{oc}}^{p}$ is not large enough, provider $p$ may have to return all of the subsidy back to the government. At the other extreme, provider $p$ may keep all of the subsidy amount, $\xi_{p}$, if it did serve sufficient number of foreign customers.

The third term in (5.7) quantifies the sum of investments provider $p$ makes from its subsidy. Provider $p$ can choose which regions to invest more. It can further decide whether to spend all of the subsidy it received, $\xi_{p}$, from the government. In this model, the provider takes some risk by spending the subsidy money. It needs to wisely decide on which regions to invest. The ideal situation for the provider is to invest into those regions in which more outside calls become possible to serve. This will motivate the providers to invest into more congested areas and thus serve the larger goal of improving user-perceived link quality.

Remark 1: Providers cannot be hurt by the subsidization option.

A provider (say $p$ ) does not have to take the subsidization option if it will not generate any profits. If $p$ does not participate in the subsidization plan, then the government cannot penalize it for not serving the required number of outside calls and therefore the revenue generated from the provider's own investment $E_{p}$ will remain intact.

Remark 2: Providers can easily be motivated into a subsidized market.

The government can offer a large $\xi_{p}$ and a conservative (i.e., not heavily penalizing) penalty function to attract the providers into the subsidization option. Since, from Remark 1, there is really not much of risk from the subsidization option, the tipping point for providers to sign into subsidization contracts will not be high. A relatively high $\xi_{p}$ will promise a positive return from the subsidization.

Remark 3: Providers will be motivated to invest in a non-overlapping manner and collectively cover a larger area.

The number of outside calls $N_{\mathrm{oc}}^{p}$ in (5.7) depends on the amount of overlap between the network coverage areas of different providers. Operators, by not duplicating their infras- 
tructure in the same areas, can minimize their investment cost $s_{p r}$, while at the same time minimizing the penalty cost $\left(1-\mathcal{T}\left(N_{\mathrm{oc}}^{p}\right)\right) \xi_{p}$ charged by the government in (5.7), yielding high revenues. This observation agrees with the conclusions in [77], in which the analysis suggests that minimization of the duplication of network infrastructure by different providers yields higher provider revenues.

Remark 4: As the ratio $\frac{\alpha}{\beta}$ increases, subsidization will be more beneficial, particularly to those providers smaller in size.

Suppose $\alpha$ increases (and $\beta$ remains the same, so that the ratio $\frac{\alpha}{\beta}$ increases). Then from (5) and (6), it follows that $N_{\mathrm{oc}}^{p}$ will also increase. This will in turn increase the contribution of the second term in (5.7) to provider $p$ 's revenue. An interesting situation happens for smaller providers. Small providers serve small regions, so their customers are more apt to leave their home regions. Hence they make relatively more outside calls than home calls. Therefore, the contribution of the second and third term of (5.7) into their revenue total will be more than the first term's contribution for small providers.

Remark 5: Small providers will be more able to compete with large providers as the ratio $\frac{\alpha}{\beta}$ increases.

This follows from Remark 4. A new note to make here is that small providers with smaller infrastructure will be able to compete against providers with large infrastructure as long as $\alpha$ is relatively more than $\beta$. They will be able to do so by swiftly investing into congested spots where larger providers cannot reach well.

Remark 6: Monopolization will be avoided as long as the ratio $\frac{\alpha}{\beta}$ is high.

This follows from Remark 4 and Remark 5. As we've seen, under this scenario smaller providers have the advantage. Thus, there will be no incentive to monopolize.

\subsection{Perfect Nash Equilibrium}

As stated previously, our game is one where the government moves first followed by the providers. This makes sense intuitively, as it is natural that the government defines the rules and private companies then react to them. The government strategy is to set the 
subsidy amounts $\xi_{p}$ and the bandwidths $B_{p}$ granted to each provider $p$. After this, each provider simultaneously tries to maximize its utility by optimizing its strategic variables: subsidy amounts spent in each region $s_{p r}$, bandwidth allocated in each region $b_{p r}$ and fee charged to customers $f_{p}$. The optimization method to compute the equilibrium solutions of the providers' problems is illustrated in Figure 5.2 by assuming two providers and two regions for simplicity. Game theoretically, the game tree has two types of subtree: the whole game itself, and also any subtree occurring after the government makes its initial move.

In game theory, a Nash equilibrium is a set of strategies, one for each player, with the property that: given the other players stick to their assigned strategies, no player can gain by unilaterally deviating from their own strategy. A perfect Nash equilibrium [138] is a special type of Nash equilibrium which has this property not only with regard to the whole game, but also if restricted (in the natural way) to any subtree of the game.

In our game, a strategy for the government can be represented as $g=\left\{\xi_{p}, B_{p}\right\}_{p \in \mathcal{P}}$. Denote the set of all such strategies by $G$. Next, a strategy for provider $p \in \mathcal{P}$ can be represented by $a_{p}(g)=\left\{s_{p r}(g), b_{p r}(g), f_{p}(g)\right\}_{r \in \mathcal{R}}$. Note that the strategies for the providers have $g$ as an argument, as the providers move after the government and so can base their moves according to what the government does. Finally, denote the strategies of all the providers collectively by the vector $\boldsymbol{a}(g)=\left\{a_{p}(g)\right\}_{p \in \mathcal{P}}$.

Given the above notations, the definition of perfect Nash equilibrium reduces to the following.

Definition 1. A set of strategies $\left\{\boldsymbol{a}^{*}, g^{*}\right\}$ constitutes a perfect Nash equilibrium of this game, if and only if, it satisfies the following set of inequalities:

$$
\begin{aligned}
& U_{p}\left(a_{p}^{*}(g), \boldsymbol{a}_{-p}^{*}(g), g\right) \geq U_{p}\left(a_{p}(g), \boldsymbol{a}_{-p}^{*}(g), g\right), \forall a_{p}, p \in \mathcal{P}, \text { and } g \in G \\
& \text { and } U_{\mathrm{G}}\left(g^{*}, \boldsymbol{a}^{*}\left(g^{*}\right)\right) \geq U_{\mathrm{G}}\left(g, \boldsymbol{a}^{*}(g)\right) \forall g \in G,
\end{aligned}
$$

where, $\boldsymbol{a}_{-p}^{*}=\left\{a_{i}\right\}_{i \in \mathcal{P}, i \neq p}$, is the vector of strategies of all the providers other than $p$. The function $U_{p}$ is the provider $p$ 's utility given by the expression in maximization problem of 


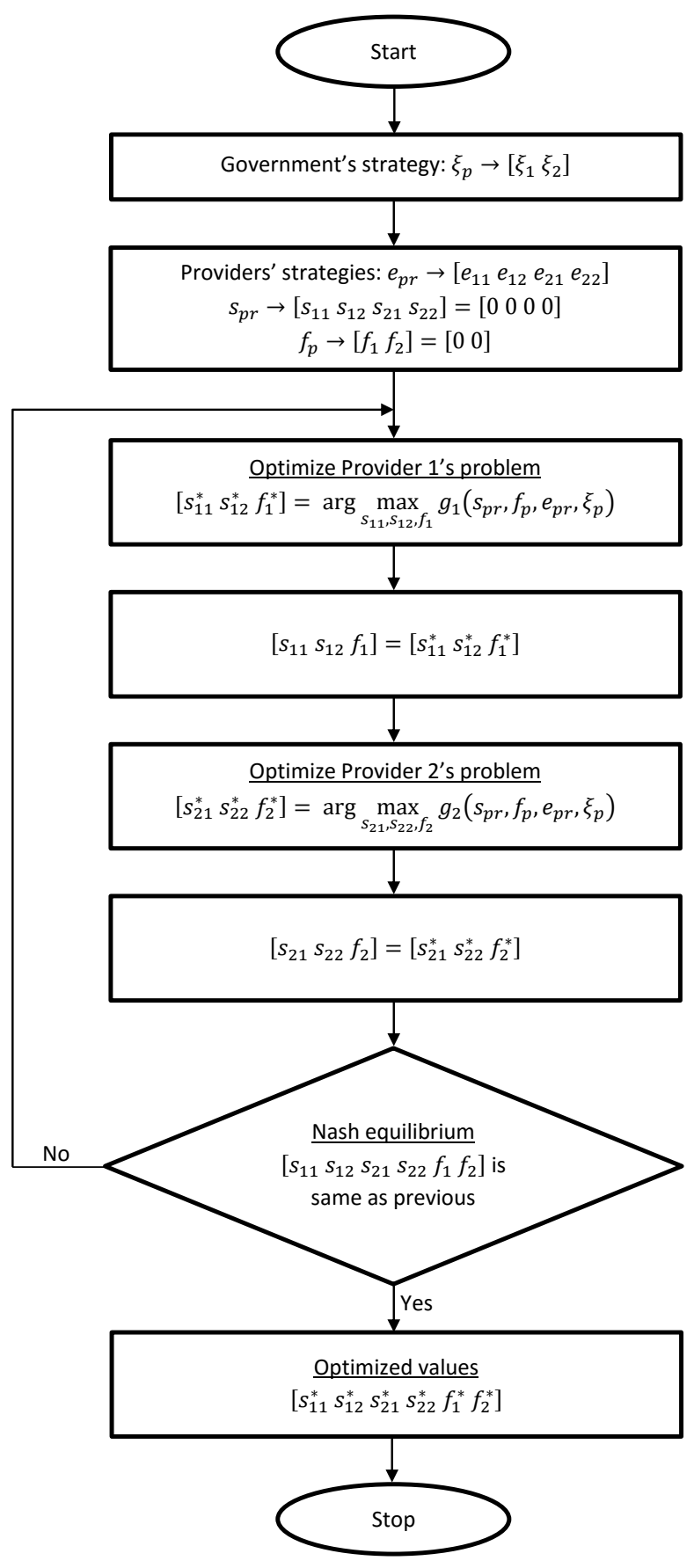

Figure 5.2: Flowchart of the iterative optimization method for finding optimized values of the subsidy amounts to be spent by the providers in different regions, and the optimum fee to be charged by the providers to the customers. 
(5.7). The function $U_{\mathrm{G}}$ is the government's utility given by the expression in maximization problem of (5.12).

In general, for our game the conditions (16) are too difficult to solve analytically, or even computationally. To gain insights, we consider a simplified form of the game in which a) there are only two regions and two providers; b) the bandwidth variable is taken out of the model; and c) there is a linear penalty function. Assuming particular parameter values, we can computationally solve for the providers' equilibrium strategies as a function of $g$; from this we argue that a perfect Nash equilibrium must exist (Section 5.5.3).

\subsection{A Simplified Spectrum Market}

In this section, we simplify the market model in Section 5.2, in order to have tractable expressions that can facilitate numerical evaluations. In particular, we make the following assumptions:

- We assume a linear penalty function $\mathcal{T}\left(N_{\mathrm{oc}}^{p}\right)$ in $(5.7)$, for which $\mathcal{T}(0)=1$ (if a provider serves no foreign customers, it loses all of its subsidy). It implies that the (1$\left.\mathcal{T}\left(N_{\mathrm{oc}}^{p}\right)\right) \xi_{p}$ term in (7) can be recast as a reward function $\delta N_{\mathrm{oc}}^{p}$, where $\delta$ is a peroutside-call reward that the government gives to providers.

- We eliminate the explicit handling of bandwidth in (5.3) and assume that it is implicit in the intensity function that expresses the received signal quality on an access link. Hence, (5.3) reduces to $\psi_{p r}=Q\left(s_{p r}+e_{p r}\right)$.

- We assume a concave (square root) utility function $u$ in (5.1), and linear signal quality function $Q$.

- We then consider a particular case with two-regions and two-providers to analyze the providers' part of the equilibrium solution (subsidy $s_{p r}^{*}$ and fee $f_{p}^{*}$ ), as a function of the government's strategy $\xi$.

These assumptions will be described in further detail in the following subsections. 


\subsubsection{A Linear Penalty Function}

The linear penalty function described above is illustrated in Figure 5.3(a) which is a linearly decreasing function of $N_{\mathrm{oc}}^{p}$. If provider $p$ serves a minimum of $N_{\mathrm{oc}, \text { th }}^{p}$ number of outside calls, then the penalty to the provider will be zero. On the other hand, the reward function

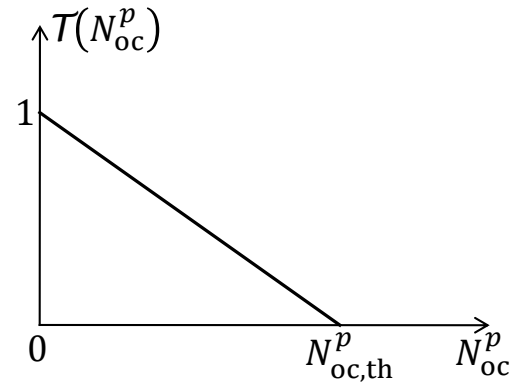

(a) Penalty function

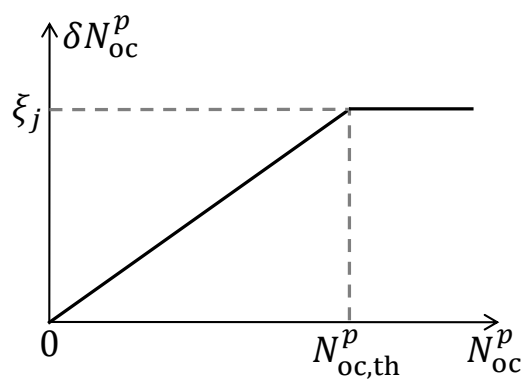

(b) Reward function

Figure 5.3: Illustration of penalty and reward functions.

illustrated in Figure 5.3(b) is an increasing function of $N_{\mathrm{oc}}^{p}$. If provider $p$ serves a minimum of $N_{\mathrm{oc}, \mathrm{th}}^{p}$ number of outside calls, then it would receive complete subsidy amount $\xi_{p}$, which is equivalent to zero penalty. Here, the slope of reward function is the per-outside-call reward $\delta$ that is specified by the government. Based on the available budget $\xi$ and the expected number of outside calls $\alpha N_{\mathrm{C}}$, government can determine the per-outside-call reward as

$$
\delta=\frac{\xi}{\alpha N_{\mathrm{C}}}=\frac{\sum_{p=1}^{N_{\mathrm{P}}} \xi_{p}}{\alpha \sum_{r=1}^{N_{\mathrm{R}}} n_{r}} .
$$

Then, provider $p$ has to serve $N_{\mathrm{oc}, \mathrm{th}}^{p}=\xi_{p} / \delta$ number of outside calls to receive the maximum reward $\xi_{p}$.

\subsubsection{Bandwidth Implicit in the Intensity Function}

As stated earlier, we assume $\psi_{p r}=Q\left(s_{p r}+e_{p r}\right)$ as a simplified version of (5.3). Using this, the linear form for the penalty function, and the expressions (5.2)-(5.6) and (5.17), we may 
rewrite provider $p$ 's problem in (5.7) as follows:

$$
\begin{aligned}
& \max _{s_{p 1}, s_{p 2}, \ldots, s_{p N_{\mathrm{R}}, f_{p}}} \sum_{r=1}^{N_{\mathrm{R}}} \frac{f_{p} n_{r}\left(\beta u\left(Q\left(s_{p r}+e_{p r}\right)\right)-f_{p}\right)}{\sum_{p^{\prime}=1}^{N_{\mathrm{P}}} \beta u\left(Q\left(s_{p^{\prime} r}+e_{p^{\prime} r}\right)\right)-f_{p^{\prime}}}-\sum_{r=1}^{N_{\mathrm{R}}}\left(s_{p r}+e_{p r}\right) \\
& +\frac{\xi}{N_{\mathrm{C}}} \sum_{r=1}^{N_{\mathrm{R}}} \sum_{\hat{r} \neq r} \frac{n_{\hat{r}} n_{r}}{\sum_{r^{\prime} \neq \hat{r}} n_{r^{\prime}}} \frac{u\left(Q\left(s_{p r}+e_{p r}\right)\right)}{\sum_{p^{\prime}=1}^{N_{\mathrm{P}}} u\left(Q\left(s_{p^{\prime} r}+e_{p^{\prime} r}\right)\right)} \times\left(1-\frac{\beta u\left(Q\left(s_{p \hat{r}}+e_{p \hat{r}}\right)\right)-f_{p}}{\sum_{p^{\prime}=1}^{N_{\mathrm{P}}} \beta u\left(Q\left(s_{p^{\prime} \hat{r}}+s_{p^{\prime} \hat{r}}\right)\right)-f_{p^{\prime}}}\right) \\
& \text { s.t., } \quad \sum_{r=1}^{N_{\mathrm{R}}} s_{p r} \leq \xi_{p}, \\
& \quad s_{p r} \geq 0, r=1 \ldots N_{\mathrm{R}}, \\
& f_{p} \geq 0 .
\end{aligned}
$$

Here, it is worthwhile to note that (5.18) is independent of the parameter $\alpha$ due to the use of linear penalty function and the assumption that each customer makes same number of outside calls (i.e., $\alpha$ ).

\subsubsection{Further Simplifying Assumptions}

We further make the following simplifying assumptions for our market:

- Concave customer utility function of the form: $u(x)=\gamma \sqrt{x}$, where $\gamma \ll 1$ is a scaling factor.

- A linear signal quality function: $\psi_{p r}=Q\left(s_{p r}\right)=m_{p r}\left(s_{p r}+e_{p r}\right)$.

In general, a concave (square-root) utility in the first assumption is considered realistic enough to capture the diminishing returns behavior of received quality. The second assumption on linear signal quality function may be considered optimistic, since the signal quality will also behave in a diminishing returns manner as the investment on the infrastructure increases. However, since $Q(\cdot)$ always feeds into $u(\cdot)$ in our formulation, the $u(Q(\cdot))$ will still behave according to diminishing returns. 


\subsubsection{Two Regions and Two Providers}

To make our model more understandable, we now rewrite the providers' problems in (5.18)(5.21) for the case with two providers and two regions. All of the assumptions in Sections 5.4.1 through 5.4.3 are incorporated in the following formulations.

\subsubsection{Provider 1's Problem}

By applying the aforementioned simplifying assumptions into (5.18), the objective function of provider 1 can be expressed as:

$$
\begin{aligned}
& \max _{s_{11}, s_{12}, f_{1}} f_{1} n_{1} \frac{U_{11}}{U_{11}+U_{21}}+f_{1} n_{2} \frac{U_{12}}{U_{12}+U_{22}}+\frac{\xi n_{1}}{N_{\mathrm{C}}} \frac{\sqrt{\psi_{12}}}{\sqrt{\psi_{12}}+\sqrt{\psi_{22}}} \frac{U_{21}}{U_{11}+U_{21}} \\
& +\frac{\xi n_{2}}{N_{\mathrm{C}}} \frac{\sqrt{\psi_{11}}}{\sqrt{\psi_{11}}+\sqrt{\psi_{21}}} \frac{U_{22}}{U_{12}+U_{22}}-s_{11}-s_{12}, \\
& s_{11}, s_{12}, f_{1} \geq 0
\end{aligned}
$$

where $U_{p r}=\gamma \beta \sqrt{m_{p r}\left(s_{p r}+e_{p r}\right)}-f_{p}$. In order to find the solutions $s_{11}^{*}, s_{12}^{*}$ and $f_{1}^{*}$ which maximize the provider 1's problem in (5.22), we take the first-order conditions for the system (5.22)-(5.24) as follows:

w.r.t. $f_{1}$ :

$$
\begin{aligned}
& \frac{n_{1}\left(U_{11}-f_{1}\right)\left(U_{11}+U_{21}\right)+f_{1} n_{1} U_{11}}{\left(U_{11}+U_{21}\right)^{2}}+\frac{n_{2}\left(U_{12}-f_{1}\right)\left(U_{22}+U_{12}\right)+f_{1} n_{2} U_{12}}{\left(U_{22}+U_{12}\right)^{2}} \\
& \quad+\frac{\xi n_{1}}{N_{\mathrm{C}}} \frac{\sqrt{\psi_{12}}}{\sqrt{\psi_{12}}+\sqrt{\psi_{22}}} \frac{U_{21}}{\left(U_{11}+U_{21}\right)^{2}}+\frac{\xi n_{2}}{N_{\mathrm{C}}} \frac{\sqrt{\psi_{11}}}{\sqrt{\psi_{11}}+\sqrt{\psi_{21}}} \frac{U_{22}}{\left(U_{12}+U_{22}\right)^{2}}=0
\end{aligned}
$$

w.r.t. $s_{11}$ :

$$
\begin{gathered}
\frac{\beta n_{1} \gamma m_{11} U_{21}}{2 \sqrt{\psi_{11}}\left(U_{11}+U_{21}\right)^{2}}\left(f_{1}-\frac{\xi}{N_{\mathrm{C}}} \frac{\sqrt{\psi_{12}}}{\sqrt{\psi_{12}}+\sqrt{\psi_{22}}}\right)+\frac{n_{2} \xi m_{11} \sqrt{\psi_{21}}}{2 N_{\mathrm{C}}\left(\sqrt{\psi_{21}}+\sqrt{\psi_{11}}\right)^{2} \sqrt{\psi_{11}}} \frac{U_{22}}{U_{12}+U_{22}} \\
-1-\lambda_{1}=0,
\end{gathered}
$$


w.r.t. $s_{12}$ :

$$
\begin{gathered}
\frac{\beta n_{2} \gamma m_{12} U_{22}}{2 \sqrt{\psi_{12}}\left(U_{12}+U_{22}\right)^{2}}\left(f_{1}-\frac{\xi}{N_{\mathrm{C}}} \frac{\sqrt{\psi_{11}}}{\sqrt{\psi_{11}}+\sqrt{\psi_{21}}}\right)+\frac{n_{1} \xi m_{12} \sqrt{\psi_{22}}}{2 N_{\mathrm{C}}\left(\sqrt{\psi_{12}}+\sqrt{\psi_{22}}\right)^{2} \sqrt{\psi_{12}}} \frac{U_{21}}{U_{11}+U_{21}} \\
-1-\lambda_{1}=0,
\end{gathered}
$$

and

$$
\lambda_{1} \geq 0, s_{11}+s_{12}=\xi_{1} \text { or } \lambda_{1}=0, \mathrm{~s}_{11}+\mathrm{s}_{12} \leq \xi_{1} .
$$

Here, $\lambda_{1}$ is the Lagrangian multiplier for the constraint (5.23) on the total amount of subsidization the government offers to providers.

\subsubsection{Provider 2's Problem}

Similar to provider 1, the objective function of provider 2 can be expressed as

$$
\begin{aligned}
\max _{s_{21}, s_{22}, f_{2}} f_{2} n_{1} \frac{U_{21}}{U_{11}+U_{21}}+f_{2} n_{2} & \frac{U_{22}}{U_{21}+U_{22}}+\frac{\xi n_{1}}{N_{\mathrm{C}}} \frac{\sqrt{\psi_{22}}}{\sqrt{\psi_{12}}+\sqrt{\psi_{22}}}\left(\frac{U_{11}}{U_{11}+U_{21}}\right) \\
& +\frac{\xi n_{2}}{N_{\mathrm{C}}} \frac{\sqrt{\psi_{21}}}{\sqrt{\psi_{11}}+\sqrt{\psi_{21}}}\left(\frac{U_{12}}{U_{12}+U_{22}}\right)-s_{21}-s_{22},
\end{aligned}
$$

s. t., $\quad \mathrm{s}_{21}+\mathrm{s}_{22} \leq \xi_{2}, \quad$ and $\quad \mathrm{s}_{21}, \mathrm{~s}_{22}, \mathrm{f}_{2} \geq 0$.

Again similar to Provider 1, Provider 2 will have to optimize w.r.t. $s_{21}, s_{22}$, and $f_{2}$. So, the first-order conditions will be:

w.r.t. $f_{2}$ :

$$
\begin{aligned}
& \frac{n_{1}\left(U_{21}-f_{2}\right)\left(U_{11}+U_{21}\right)+f_{2} n_{1} U_{21}}{\left(U_{11}+U_{21}\right)^{2}}+\frac{n_{2}\left(U_{22}-f_{2}\right)\left(U_{12}+U_{22}\right)+f_{2} n_{2} U_{22}}{\left(U_{12}+U_{22}\right)^{2}} \\
& +\frac{\xi n_{1}}{N_{\mathrm{C}}} \frac{\sqrt{\psi_{22}}}{\sqrt{\psi_{12}}+\sqrt{\psi_{22}}} \frac{U_{11}}{\left(U_{11}+U_{21}\right)^{2}}+\frac{\xi n_{2}}{N_{\mathrm{C}}} \frac{\sqrt{\psi_{21}}}{\sqrt{\psi_{11}}+\sqrt{\psi_{21}}} \frac{U_{12}}{\left(U_{12}+U_{22}\right)^{2}}=0
\end{aligned}
$$


w.r.t. $s_{21}$ :

$$
\begin{gathered}
\frac{\beta n_{1} \gamma m_{21} U_{11}}{2 \sqrt{\psi_{21}}\left(U_{11}+U_{21}\right)^{2}}\left(f_{2}-\frac{\xi}{N_{\mathrm{C}}} \frac{\sqrt{\psi_{22}}}{\sqrt{\psi_{12}}+\sqrt{\psi_{22}}}\right)+\frac{n_{2} \xi m_{21} \sqrt{\psi_{11}}}{2 N_{\mathrm{C}}\left(\sqrt{\psi_{21}}+\sqrt{\psi_{11}}\right)^{2} \sqrt{\psi_{21}}} \frac{U_{12}}{U_{12}+U_{22}} \\
-1-\lambda_{2}=0,
\end{gathered}
$$

w.r.t. $s_{22}$ :

$$
\begin{gathered}
\frac{\beta n_{2} \gamma m_{22} U_{12}}{2 \sqrt{\psi_{22}}\left(U_{12}+U_{22}\right)^{2}}\left(f_{2}-\frac{\xi}{N_{\mathrm{C}}} \frac{\sqrt{\psi_{21}}}{\sqrt{\psi_{11}}+\sqrt{\psi_{21}}}\right)+\frac{n_{1} \xi m_{22} \sqrt{\psi_{12}}}{2 N_{\mathrm{C}}\left(\sqrt{\psi_{12}}+\sqrt{\psi_{22}}\right)^{2} \sqrt{\psi_{22}}} \frac{U_{11}}{U_{11}+U_{21}} \\
-1-\lambda_{2}=0,
\end{gathered}
$$

and

$$
\lambda_{2} \geq 0, s_{21}+s_{22}=\xi_{2} \text { or } \lambda_{2}=0, \mathrm{~s}_{21}+\mathrm{s}_{22} \leq \xi_{2},
$$

where $\lambda_{2}$ is the Lagrangian multiplier.

\subsubsection{Government's Problem}

By assuming number of providers $N_{p}=2$, number of regions $N_{r}=2$, and eliminating the explicit handling of bandwidth $B_{p}$ in the government's objective function of (5.12), the government's problem can be rewritten as

$$
\begin{aligned}
\max _{\xi_{1}, \xi_{2}} & n_{1} \beta \gamma\left(\frac{\sqrt{\psi_{11}} U_{11}+\sqrt{\psi_{21}} U_{21}}{U_{11}+U_{21}}\right)+n_{2} \beta \gamma\left(\frac{\sqrt{\psi_{12}} U_{12}+\sqrt{\psi_{22}} U_{22}}{U_{12}+U_{22}}\right) \\
& +\frac{\alpha \gamma n_{2}}{\sqrt{\psi_{11}}+\sqrt{\psi_{21}}}\left(\frac{\psi_{21} U_{12}+\psi_{11} U_{22}}{U 12+U_{22}}\right)+\frac{\alpha \gamma n_{1}}{\sqrt{\psi_{12}}+\sqrt{\psi_{22}}}\left(\frac{\psi_{22} U_{11}+\psi_{12} U_{21}}{U_{11}+U_{21}}\right)
\end{aligned}
$$

s.t., $\quad \xi_{1}+\xi_{2} \leq \xi$,

$\xi_{1}, \xi_{2} \geq 0$ 


\subsubsection{Optimum Subsidy Amounts $\left(\mathrm{s}_{\mathrm{pr}}^{*}\right)$ and Optimum Fee $\left(\mathrm{f}_{\mathrm{p}}^{*}\right)$}

Looking at the formulations of the providers' problems described in (5.22)-(5.34), it is clear that the problem in hand is a multi-criteria maximization problem with (5.22) and (5.29) being the two simultaneous objective functions. The parameters to be optimized

are the fees $f_{1}, f_{2}$, and the subsidy amounts $s_{11}, s_{12}, s_{21}, s_{22}$, which are functions of the input parameters $\beta, n_{1}, n_{2}, \xi_{1}$ and $\xi_{2}$. We wish to solve the eight simultaneous equations formed by the first order conditions in (5.25)-(5.28) and (5.31)-(5.34). Note that each parameter to be optimized depends on the other optimization parameters. Due to high number of unknown variables and the involved complexity while solving these simultaneous equations (such as solving polynomials of 7 th order or higher), it is impractical to derive the expressions for optimum parameters in closed form. Hence, we numerically solve the simultaneous equations of (5.25)-(5.28) and (5.31)-(5.34) to obtain the solutions $s_{p r}^{*}$ and $f_{p}^{*}$

\subsection{Numerical Results}

In Section 5.4.4, a simplified model with two providers and two regions was considered in which the goal was to jointly maximize the two providers' objective functions (5.22) and (5.29) by optimizing the subsidy amounts $s_{11}, s_{12}, s_{21}$ and $s_{22}$, and the fees $f_{1}$ and $f_{2}$. In this section, we first show the convergence of our simulations for the case of two regions and two providers. Then, we analyze the characteristics of this model using the numerical equilibrium solutions.

\subsubsection{Simulation Convergence to Equilibrium}

Due to complexity of the providers' problems represented in (5.22) and (5.29), an analytical proof for the existence of a Nash equilibrium was deemed infeasible. However, our extensive simulations of the game converged and were based on best response algorithm. Therefore, we guarantee that the simulations converge to an equilibrium. A two player game based 

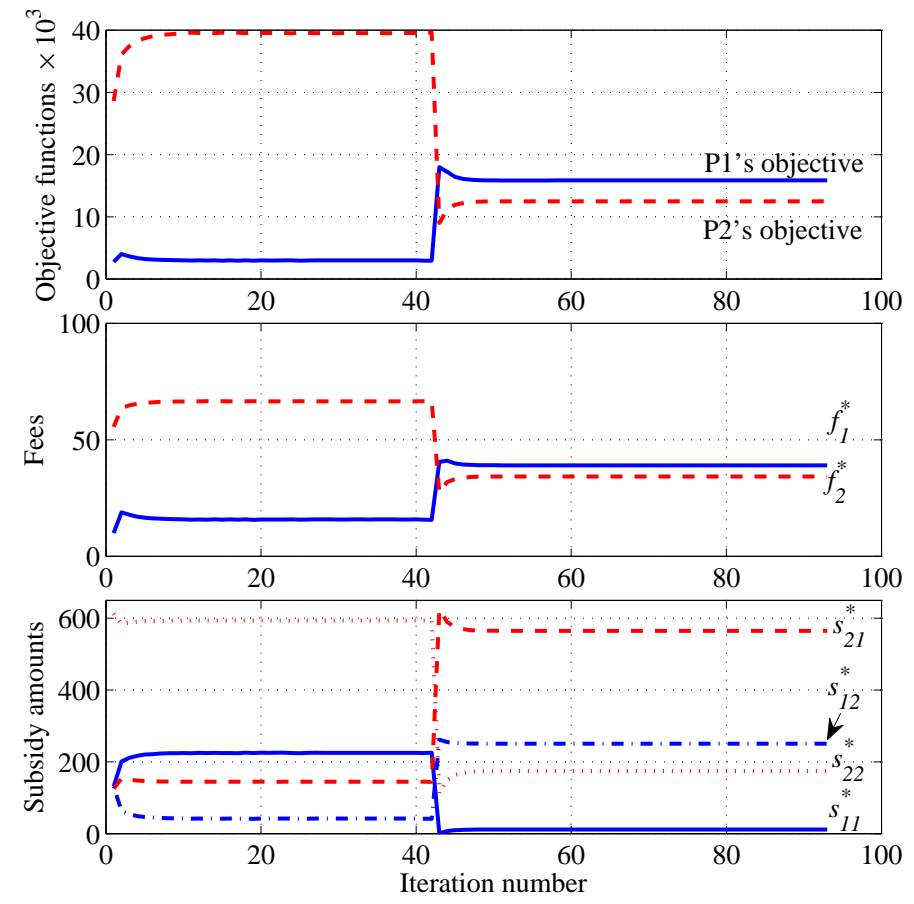

Figure 5.4: The convergence to equilibrium using best response algorithm. System parameters: $\xi_{1}=262, \xi_{2}=738, \beta=76, n_{1}=26, n_{2}=744, \gamma=0.05, \epsilon=10^{-3}$.

on the best response algorithm is designed in Matlab for the case of two providers and two regions. During each iteration of the algorithm,

1. Provider 1's parameters $s_{11}, s_{12}$ and $f_{1}$ are optimized by solving the simultaneous equations (5.25)-(5.28);

2. Provider 2's parameters $s_{21}, s_{22}$ and $f_{2}$ are optimized by solving the simultaneous equations (5.31)-(5.34);

3. The optimized parameters evaluated in the previous two steps are used in (5.22) and (5.29) to compute the providers' objective values.

To check for convergence, the evaluated objective values in the current iteration are compared to their respective values in the previous iteration. If the differences are smaller than a threshold $\epsilon$, convergence is attained.

The best response simulator described above was run with arbitrary system parameter settings and the simulator took 93 iterations to converge. Behavior of the optimization 
Table 5.2: Statistics of the number of iterations for convergence.

\begin{tabular}{|l|l|}
\hline Iterations & Number of occurrences \\
\hline \hline$\leq 15$ & 9141 \\
\hline $16-99$ & 388 \\
\hline$\geq 100$ & 471 \\
\hline
\end{tabular}

parameters and the provider's objective functions during the iterations are shown in Figure 5.4 .

The number of iterations required for convergence can vary depending on the input parameter settings. To obtain the statistics of the number of iterations, the simulator was run 10,000 times with random system parameters. The system parameters for each iteration were generated using uniformly distributed random integers: $\beta \sim \mathcal{U}\{30,200\},\left\{n_{1}, n_{2}\right\} \sim$ $\mathcal{U}\{20,1000\}$ and $\xi_{1} \sim \mathcal{U}\{50,950\}$. The parameter $\xi_{2}$ was generated using $\xi_{2}=\xi-\xi_{1}$ so that the sum of $\xi_{1}$ and $\xi_{2}$ is equal to the subsidy budget $\xi$ of the government.

The resulting distribution of the number of iterations for convergence is described in Table 5.2. Most of the simulation runs (91.41\% of 10,000) converged within 15 iterations, while some of the simulation runs $(3.88 \%)$ took 16 to 99 iterations to converge. The remaining $4.71 \%$ of the simulation runs took 100 or more iterations to converge.

\subsubsection{Per-user Spectral Efficiency for Signal Quality Function}

In this section, the average downlink spectral efficiency (SE) of a typical user will be used as the signal quality function of the subsidy model. In Section 5.4.3, a linear signal quality function $Q\left(s_{p r}\right)=m_{p r} s_{p r}$ was assumed. In this section, through the system level simulations of a realistic radio access network, the average per-user SE will be shown to be linearly proportional to $s_{p r}+e_{p r}$. These simulation results will also be used to find the values of the proportionality constants $m_{p r}$.

Real BS locations of two providers in two cities of UK: Manchester and Leeds, were used in the system level simulations [126]. The real $B S$ locations of the two providers are as shown in Figure 5.5, and the details are provided in Table 5.3. The users' locations were 
generated using a homogeneous Poisson point process with intensity $\lambda_{\mathrm{u}}=20 \mathrm{users} / \mathrm{km}^{2}$. The following assumptions were made to simulate the average per-user SE:

- Same transmit power $46 \mathrm{dBm}$ for all BSs and every user connects to its nearest BS.

- Users with distance smaller than $d_{\min }=35 \mathrm{~m}$ from their respective BSs are discarded.

- Path-loss exponent $\delta=4$ and Rayleigh fading channel.

- All the users in a cell are served in round-robin fashion.
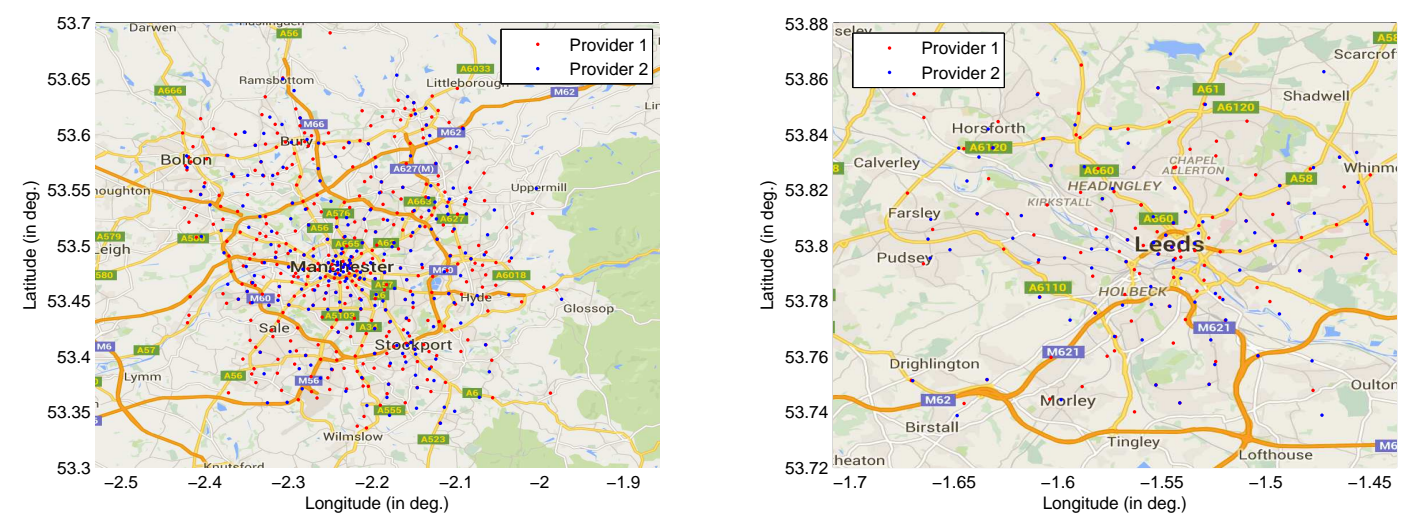

Figure 5.5: Real BS locations of 2 providers in the cities of (a) Manchester, and (b) Leeds, in UK.

Table 5.3: Details of the two regions and the two providers.

\begin{tabular}{|l|c|c|}
\hline Parameter & Region 1 (Manchester) & Region 2 (Leeds) \\
\hline Area & $1200 \mathrm{~km}^{2}$ & $225 \mathrm{~km}^{2}$ \\
\hline \multirow{2}{*}{ Provider 1's No. of BSs } & 384 & 119 \\
& $\left(0.32 \mathrm{BSs} / \mathrm{km}^{2}\right)$ & $\left(0.53 \mathrm{BSs} / \mathrm{km}^{2}\right)$ \\
\hline Provider 2's No. of BSs & 228 & 92 \\
& $\left(0.19 \mathrm{BSs} / \mathrm{km}^{2}\right)$ & $\left(0.41 \mathrm{BSs} / \mathrm{km}^{2}\right)$ \\
\hline
\end{tabular}

The purpose of the system level simulations is to obtain the plots of average per-user SE from provider $p$ in region $r$ as a function of the subsidy amount $s_{p r}$. Assuming that a fixed cost $c_{\mathrm{bs}}=10$ is associated in setting up a BS, the number of BSs that provider $p$ can setup in region $r$ is given by

$$
N_{\mathrm{bs}}(p, r)=\left\lfloor\frac{s_{p r}+e_{p r}}{c_{\mathrm{bs}}}\right\rfloor
$$


The average per-user SE computed through the simulations are plotted against the number of BSs in Figure 5.6(a). In each iteration of the simulations, $N_{\mathrm{bs}}$ BSs were randomly selected in region $r$ from the available set of BSs that are shown in Figure 5.5. For each plot in Figure 5.6(a), the maximum value for $N_{\mathrm{bs}}$ is set according to the number of BSs shown in Table 5.3.

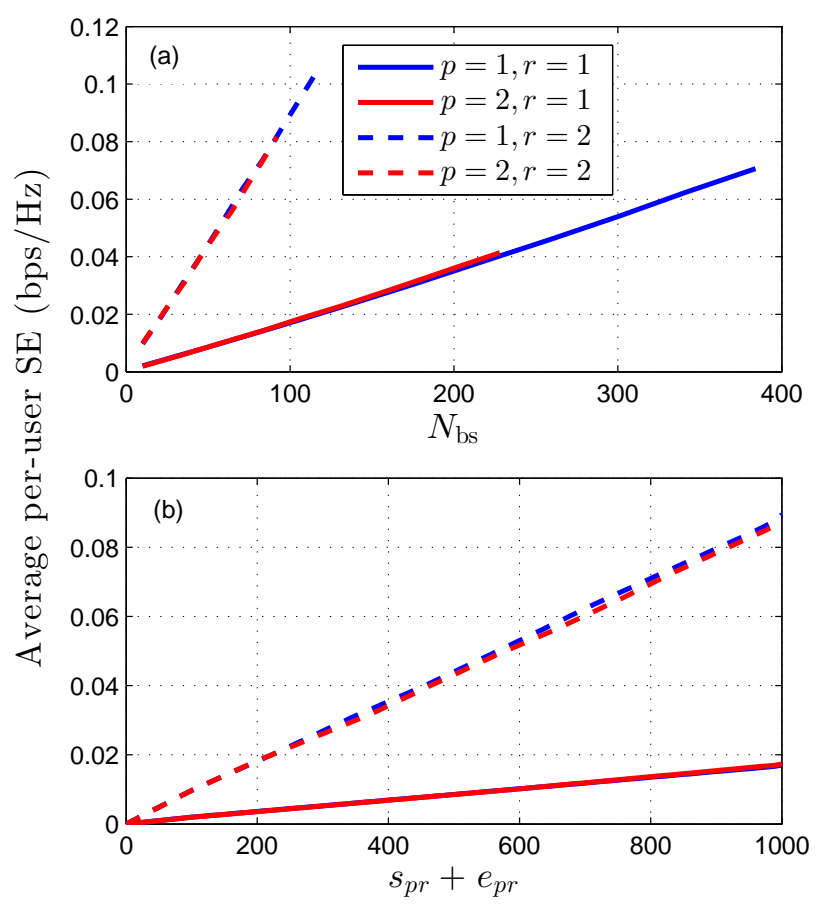

Figure 5.6: Average per-user SE as a function of (a) number of BSs of provider $p$ in region $r$, and (b) subsidy amount spent by provider $p$ in region $r$.

Using the relationship in (5.38) and linear extrapolation of the plots in Figure 5.6(a), the average per-user SE is plotted in Figure 5.6(b) as a function of $s_{p r}+e_{p r}$, the total amount invested by provider $p$ in region $r$. It can be seen in Figs. 5.6(a) and 5.6(b) that the average per-user SE is linearly proportional to $N_{\mathrm{bs}}$ and to $s_{p r}+e_{p r}$. The plots for $r=2$ have greater slopes than the plots for $r=1$ because region 2 has higher BS density than region 1 as shown in Table 5.3. Using the curve fitting tool in Matlab, the linear proportionality constant of the plots were computed as $m_{11}=1.696 \times 10^{-5}, m_{12}=8.862 \times 10^{-5}, m_{21}=1.712 \times 10^{-5}$, and $m_{22}=8.673 \times 10^{-5}$, which will be used in the signal quality function of the subsidization model. 
Table 5.4: Simulation parameters for subsidization model.

\begin{tabular}{|c|l|}
\hline Parameter & value \\
\hline$\alpha$ & 30 \\
\hline$\beta$ & 20 \\
\hline$n_{1}, n_{2}$ & 24000,4500 \\
\hline$e_{11}, e_{12}, e_{21}, e_{22}$ & $3840,1190,2280,920$ \\
\hline$\xi$ & 1000 \\
\hline$\gamma$ & 0.1 \\
\hline
\end{tabular}

\subsubsection{Numerical Optimization Results}

The simulation parameters for the subsidization model are shown in Table 5.4. Here, the number of customers $n_{r}$ in a region was calculated based on the region area shown in Table 5.3, and assuming a user density $\lambda_{\mathrm{u}}=20 \mathrm{users} / \mathrm{km}^{2}$. The real BSs shown in Figure 5.5 are assumed to be setup by the providers with their own investments $e_{p r}$. A provider's own investment $e_{p r}$ in a region was calculated based on the number of BSs in the region, and with an assumption that the cost for setting up a $\mathrm{BS}$ is $c_{\mathrm{bs}}=10$.

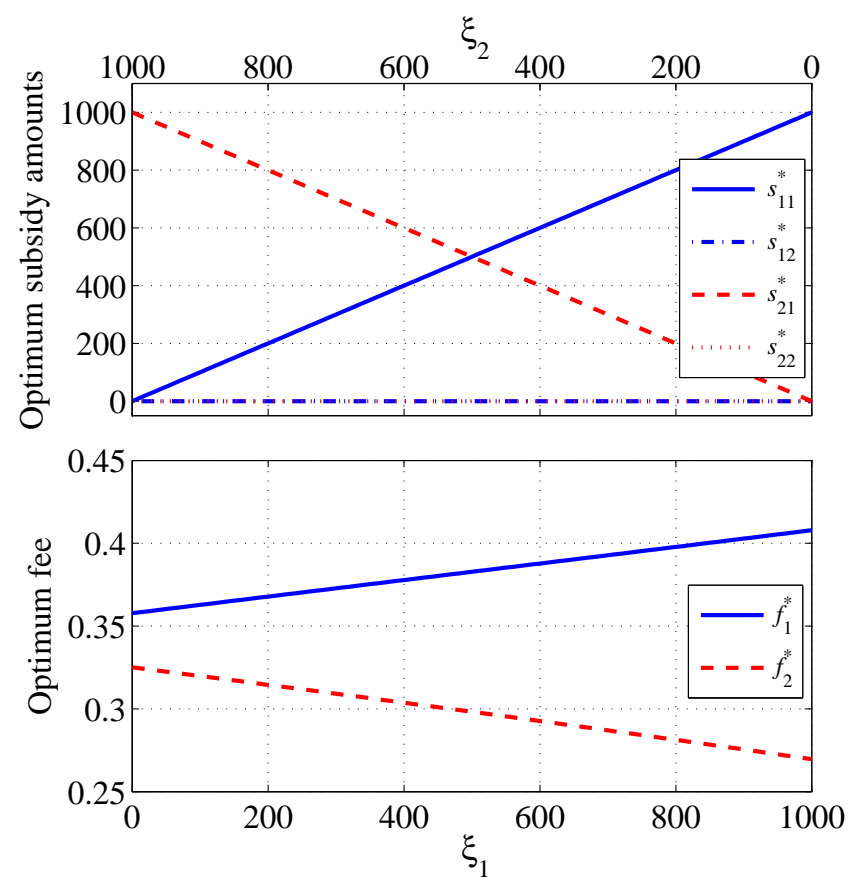

Figure 5.7: Optimum subsidy amounts spent by the providers and Optimum fee charged by the providers to each customer. 
The government splits the available subsidy budget $\xi$ between the two providers as $\xi_{1}$ and $\xi_{2}$. The optimum parameters $s_{p r}^{*}$ and $f_{p}^{*}$ are plotted as a function $\xi_{1}$ and $\xi_{2}$ in Figure 5.7 which were obtained by simultaneously solving the equations (5.25)-(5.28) and (5.31)-(5.34). It can be observed in Figure 5.7(a) that $s_{12}^{*}=s_{22}^{*}=0$, which implies that both the providers do not invest their subsidy amounts in region 2. This is because the BS density in region 2 is higher than in region 1 as shown in Table 5.3. Therefore, both the providers invest their entire subsidy amounts in region 1 as shown in Figure 5.7(a), $s_{11}^{*}=\xi_{1}$ and $s_{21}^{*}=\xi_{2}$, to increase the BS density in region 1 . The optimum fee $f_{p}^{*}$ of a provider increases with the increasing subsidy amount from the government to that provider. However, since provider 1's BS density is higher than provider 2's BS density, $f_{1}^{*}$ is greater than $f_{2}^{*}$.

Existence of Perfect Nash Equilibrium In our simplified game in Section 5.4.4, we now argue that the previous results imply the existence of a perfect Nash equilibrium (see Section 5.3). In the numerical results of Figure 5.7, the optimal strategies $s_{p r}^{*}$ and $f_{p}^{*}$ appear to be continuous functions of $\xi_{1}$ and $\xi_{2}$. Hence the government's objective function from Section 5.4.4 would be a continuous function of $\xi$. Since that problem has a compact domain, a basic theorem from analysis [139, Theorem 7, p. 142] guarantees the existence of an optimum $\xi^{*}$. This $\xi^{*}$ would then be the government's part of the perfect Nash equilibrium.

The optimum objective functions of the providers and the optimum social welfare are plotted in Figs. 5.8(a) and 5.8(b), respectively. The variation in $\xi_{1}$ and $\xi_{2}$ has considerable impact on the providers' objective functions, while not much effect on the social welfare. The social welfare maximizes when $\xi_{2}=1000$ and $\xi_{1}=0$ because provider 2 has smaller BS density as shown in Table 5.3. providing more subsidy to provider 2 increases the competition between the two providers and eventually increases the social welfare.

Optimum allocation of the government's subsidy budget $\xi$ between the two providers that maximizes the social welfare is plotted in Figure 5.9 as a function of $\xi$. For smaller government subsidy budget, allocating the entire subsidy to provider 2 maximizes the social welfare as explained in previous paragraph. However, for higher subsidy budget $(>7700$ in 

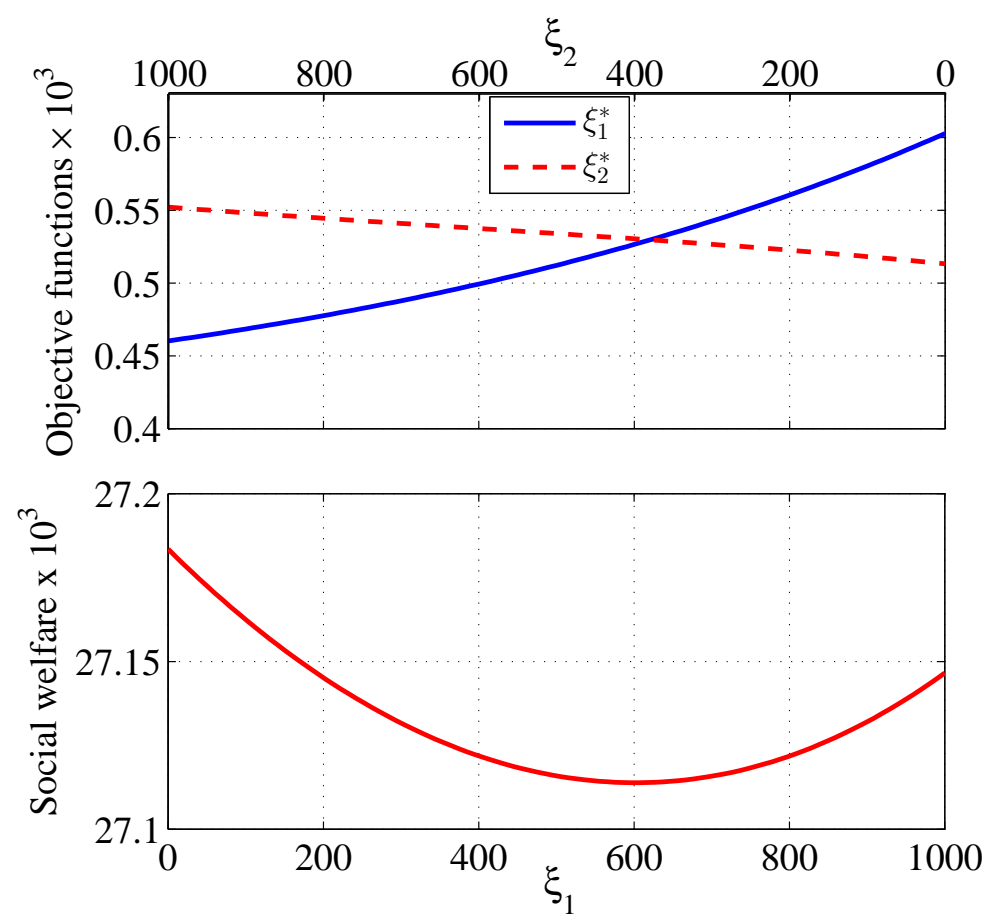

Figure 5.8: Optimum objective functions of the providers and optimum social welfare.

Figure 5.9), allocating the entire subsidy to provider 2 will over-benefit the provider 2 and hence splitting the subsidy proportionally between the providers will maximize the social welfare. The splitting proportion $\xi_{1}^{*} / \xi_{2}^{*}$ is about 0.6 because the ratio between the total number of BSs of the two providers is about 0.6.

The social welfare and per-user SE are plotted as a function of government's subsidy budget in Figure 5.10. It shows that the social welfare and the per-user SE can be increased significantly through subsidization from the government. With a government's subsidy budget of $\xi=15000$, the average per-user SE can be increased by $155 \%$. Figure 5.10 also shows the social welfare and per-user SE plots for 3 different proportions of splitting between the providers. It can be seen that these plots match closely with the optimum plots. Therefore, no matter how the government allocates the subsidy to the providers, the users receive close-to-optimum service via subsidization. 

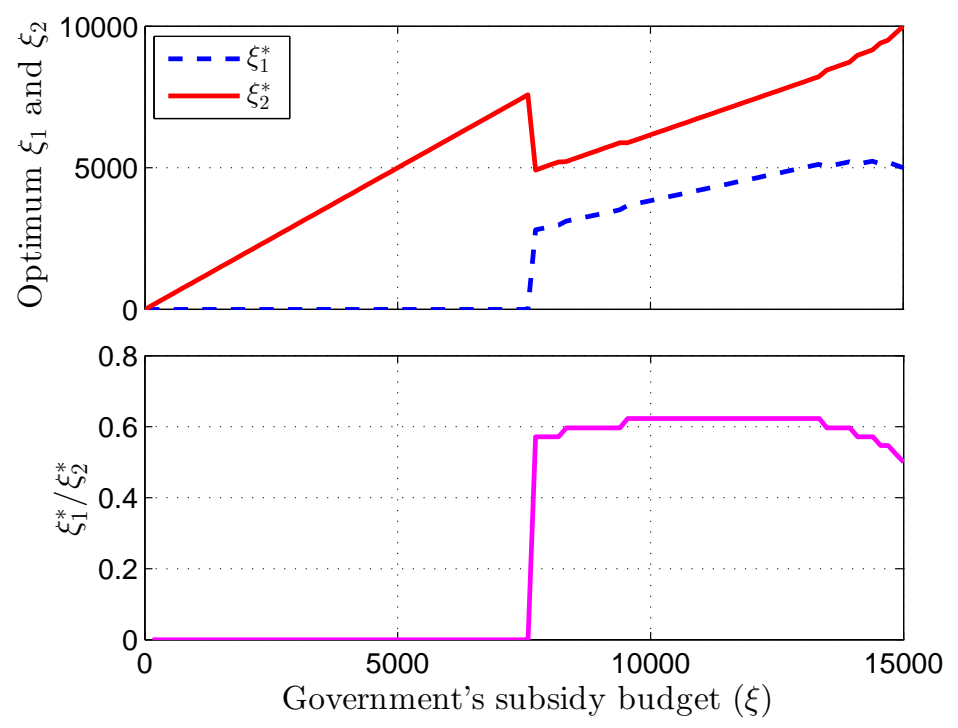

Figure 5.9: Optimum allocation of the government's subsidy budget between the providers.
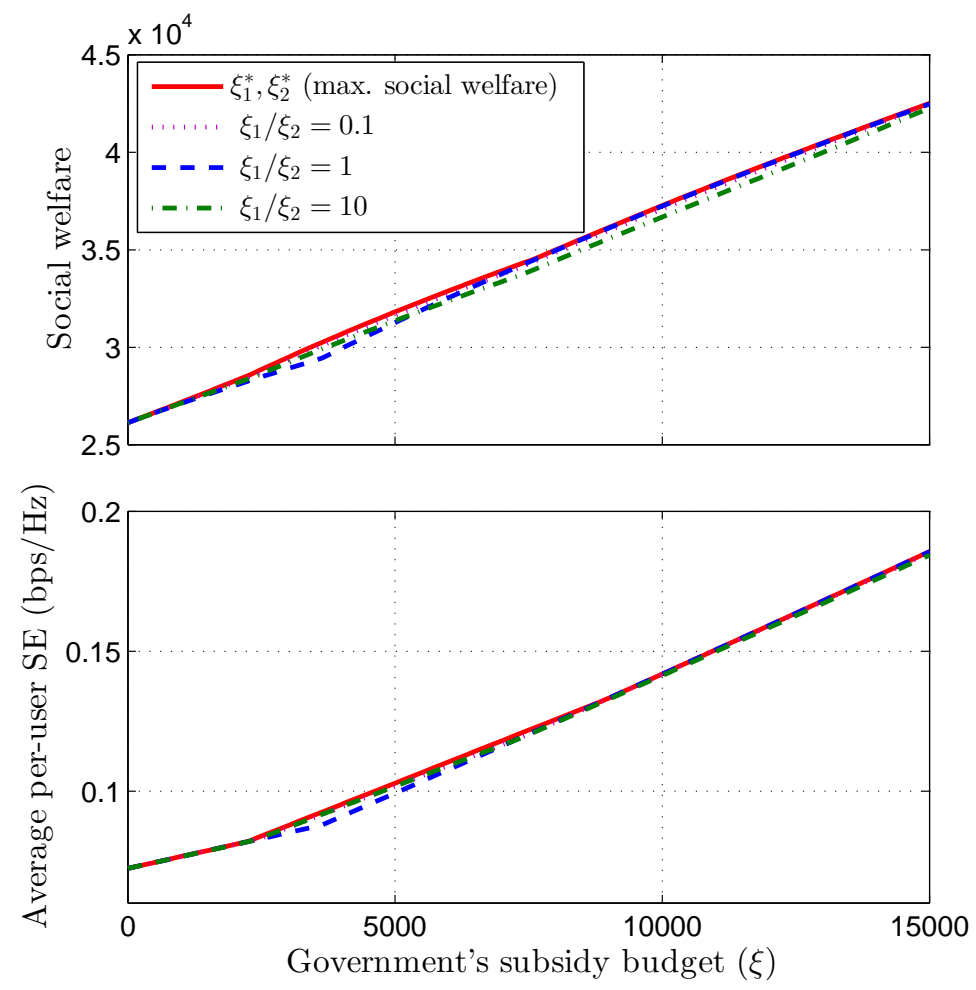

Figure 5.10: Social welfare and average per-user SE versus the government subsidy budget. 


\section{CHAPTER VI}

\section{Device-to-Device Communication and Multi-Hop Transmissions}

In wireless networks, mobile nodes with limited power budget must rely on multi-hop transmissions so as to achieve reliable communication with a distant peer device. Hence, it is of paramount importance to study the characteristics of wireless transmission over multi-hop links and its benefits over single-hop communication. In this work, a simulator is developed to assess the BER performance of multi-hop, multi-carrier communication systems. The channel model used in the simulator is developed based on the channel statistics obtained through indoor channel measurements performed using the universal software radio peripherals (USRPs). As the bandwidth supported by a USRP device is much smaller than the channel bandwidth of interest, we use a frequency domain approach to measure the impulse response of wide band channel. By using the channel measurements, we show the impact of communication distance on the delay spread and coherence bandwidth of the channel. Our simulation results with orthogonal frequency division multiplexing (OFDM) systems show that the BER performance of multi-hop communication is better than the single-hop communication. The BER performance difference between the two cases becomes more significant for wider subcarrier frequency spacing.

The goals of this chapter are: 1) to develop a frequency domain wide band channel estimation method using the universal software radio peripheral (USRP) platform that supports smaller bandwidths $(20 \mathrm{MHz})$ when compared to the channel bandwidth of interest, 2) to perform channel measurements with different distances between the transmitter and the receiver, and determine channel statistics such as path-loss, mean excess delay, root mean square (RMS) delay spread, and coherence bandwidth, 3) to build an empirical channel model as a function of distance by using the measured channel statistics, and 4) to incorporate the developed channel model into the multi-hop simulation model for evaluating the BER performance of multi-carrier systems. In the multi-hop simulation model, two devices separated by a distance $d$ communicate with each other in single-hop and two-hop scenarios. 
Using OFDM, the resulting average BERs with single and two-hop cases are compared for different distances and subcarrier frequency spacings.

\subsection{Channel Sounding Method with USRPs}

Two USRP devices from National Instruments [140] are used in the setup as shown in Figure 6.1. Each USRP is controlled by a host PC and the transmitter and receiver operations are developed using Labview software. A transmitted signal is attenuated, delayed, phase shifted, and gets corrupted with noise as it travels through the channel. The signal received by the RX USRP exhibits fading effects due to multi-path propagation.

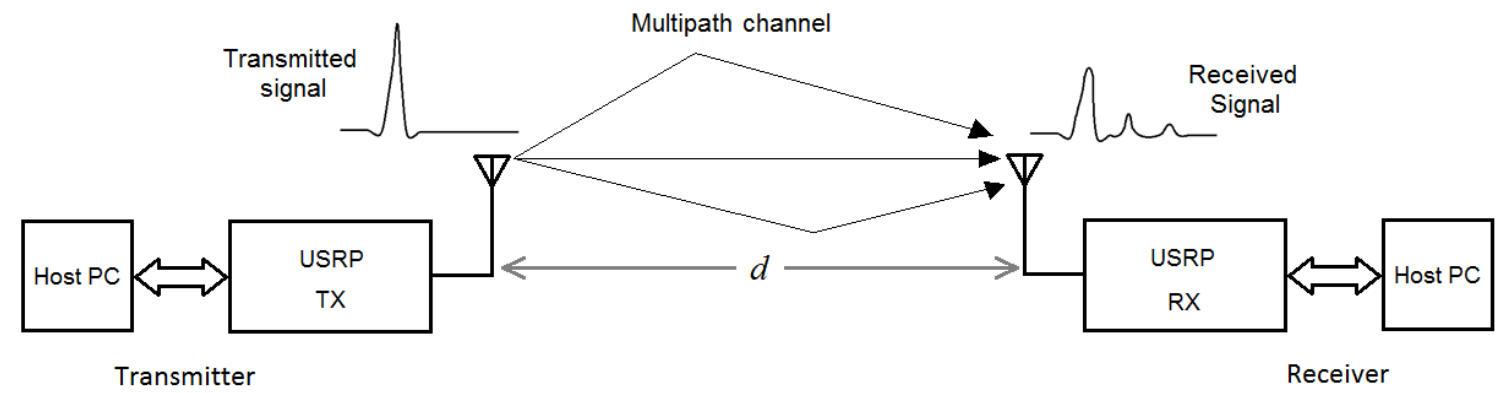

Figure 6.1: System setup for channel measurements.

\subsubsection{Channel Sounding Approach}

Channel sounding is done by transmitting $N$ narrow-band sinusoidal signals that are regularly spaced $(\Delta f)$ in the frequency domain as illustrated in Figure 6.2. Since the bandwidth of USRP is smaller than the channel bandwidth, the carrier frequency $f_{\mathrm{c} i}$ is swept across the channel bandwidth in $N$ discrete steps, $i=1,2, . ., N$. At each frequency step, a complex DC base-band signal frame consisting of 1000 samples is sent to the USRP. The USRP upconverts the DC signal to the carrier frequency $f_{\mathrm{c} i}$.

The receiver initially sets the carrier frequency to $f_{\mathrm{C} 1}$ and after receiving each frame, increments the carrier frequency by $\Delta f$. In this way, the carrier frequency sweep process at the receiver is synchronized with the transmitter. At each frequency step, beginning of the signal frame is identified via threshold detection of the received signal power. When a frame is detected and captured, it is downconverted to the base-band and sent to the host 


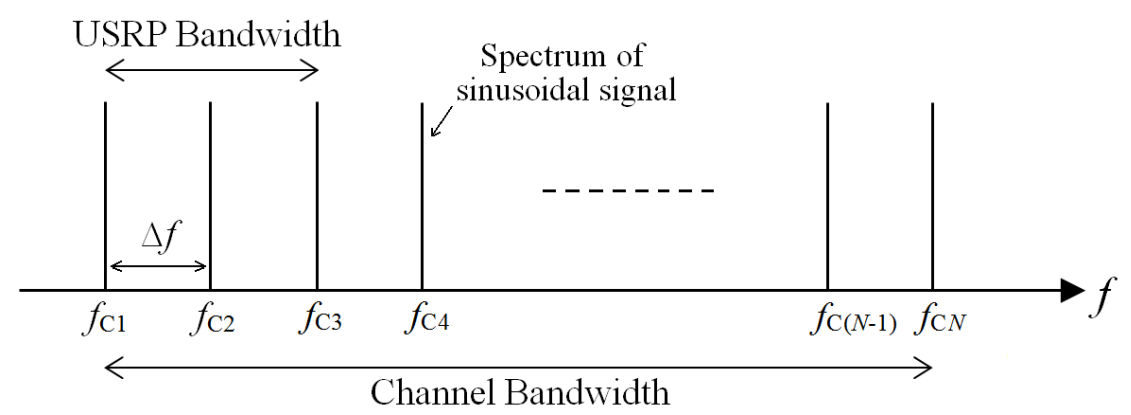

Figure 6.2: Aggregated spectrum of all the sinusoidal signals.

PC. Carrier frequency offset and phase offset corrections are performed at the host PC to obtain the complex DC signal whose magnitude and phase represents the channel response at frequency $f_{\mathrm{c} i}$.

\subsubsection{Synchronization, Sweep Rate, and Calibration}

At the receiver USRP, robust algorithms should be used to track the transmitter carrier frequency during the frequency sweep process as the transmitter and receiver USRPs use different timing references. We used power threshold detection method at the receiver's host computer to detect the signal. For longer distances between the transmitter and the receiver, dynamic range of the received power increases over the frequency band and the signal power may not be detected at some frequencies due to deep fades. In those cases, synchronization using the timing reference at the hardware level is reliable than using the host computer. Therefore, programming the FPGA in USRP to periodically change the carrier frequency can improve the sweep rate significantly. This method along with the power threshold detection of signal can improve the synchronization.

The transmitter power and receiver gain of the USRPs vary with the carrier frequency and are not calibrated. It is necessary to know these values to accurately estimate the amplitudes of received impulse response and for path-loss calculations. We used the Mixed Domain Oscilloscope to find the USRP transmit power and USRP receiver gain in the frequency band of interest. 


\subsubsection{Extracting Multipath Channel Parameters}

After capturing all the frames in a sweep, the magnitude and phase values at all the frequencies are aggregated to form the frequency response of the channel. Then, computing the inverse fast Fourier transform (IFFT) will provide the CIR $h[n]=h\left(n T_{\mathrm{S}}\right)$, where $T_{\mathrm{S}}=$ $1 /(N \Delta f)$ is the sample period and $n$ is the discrete time index. With $K$ CIR measurements, the average power delay profile (PDP) can be obtained by, $\overline{P D P}[n]=\frac{1}{K} \sum_{k=1}^{K}\left|h_{k}[n]\right|^{2}$. The mean excess delay and RMS delay spread can be respectively evaluated as

$$
\bar{\tau}=\frac{\sum_{\forall n} n T_{\mathrm{s}} \overline{P D P}[n]}{\sum_{\forall n} \overline{P D P}[n]}, \quad \bar{\tau}_{\mathrm{rms}}=\sqrt{\overline{\tau^{2}}-(\bar{\tau})^{2}}
$$

where

$$
\overline{\tau^{2}}=\frac{\sum_{\forall n}\left(n T_{\mathrm{S}}\right)^{2} \overline{P D P}[n]}{\sum_{\forall n} \overline{P D P}[n]} .
$$

Using the RMS delay spread, channel coherence bandwidth can be approximated as $B_{\mathrm{c}} \approx$ $1 /\left(5 \tau_{\text {rms }}\right)[141]$

\subsection{Channel Propagation Modeling}

The impact of large scale propagation effects on the received signal are captured by path loss (PL) and shadowing models, while the small scale propagation effects are captured by channel impulse response model.

\subsubsection{Path loss and shadowing models}

We model the average PL at a distance $d$ from the transmitter as,

$$
\overline{P L}_{\mathrm{dB}}(d)=\overline{P L}_{\mathrm{dB}}\left(d_{0}\right)+\alpha 10 \log _{10}\left(d / d_{0}\right)
$$


where, $\alpha$ is the path-loss exponent and $\overline{P L}_{\mathrm{dB}}\left(d_{0}\right)$ is the PL at a reference distance $d_{0}=1 \mathrm{~m}$ from the transmitter. Using the curve fitting tool in Matlab, we find the value of $\alpha$ that provides a best fit of (6.3) to the measured average PL values. The measured average PL values with respect to $d_{0}$ can be obtained using

$$
\overline{P L}_{\mathrm{dB}}^{\text {meas }}(d)=\overline{P L}_{\mathrm{dB}}^{\text {meas }}\left(d_{0}\right)+10 \log _{10}\left(\frac{E_{\mathrm{rx} 0}}{E_{\mathrm{rx}}(d)}\right)
$$

where, $E_{\mathrm{rx}}(d)$ and $E_{\mathrm{rx} 0}$ are the total energies received from all the multi path components (MPCs) at distances $d$ and $d_{0}$, respectively. These energies can be determined using the measured PDPs as $E_{\mathrm{rx}}(d)=\sum_{\forall n} \overline{P D P}[n]$.

Shadow fading is modeled using a log-normally distributed variable with zero mean and standard deviation $\sigma$. We denote this variable in decibel scale as $X_{\sigma} \sim \mathcal{N}(0, \sigma)$. The $\sigma$ is assumed to be independent of $d$ and its value can be found by fitting the Gaussian PDF to the histogram of the measured samples.

\subsubsection{Channel impulse response model}

Theoretically, the CIR $h(t)$ is composed of an infinite number of MPC arrivals, which can be expressed by

$$
h(\tau)=\sum_{i=1}^{\infty} a_{i} e^{j \phi_{i}} \delta\left(\tau-\tau_{i}\right),
$$

where, $\tau_{i}$ denotes the delay of the $i$ th arrival in propagation between the transmitter and the receiver. The $a_{i}$ and $\phi_{i} \sim \mathcal{U}[0,2 \pi]$ [142] represent, respectively, the amplitude and phase shift of the $i$ th arrival. It is possible to completely determine the PDP by a set of samples spaced by $\Delta \tau=1 / W$, where $W$ is the bandwidth of the channel [143] (i.e., $\left.\tau_{k}=k / W, k=0,1,2, \ldots\right)$. Each sample in the set is the power of all MPCs arrived during the delay bin $\Delta \tau$. Then, the PDP can be expressed as,

$$
\tilde{p}(\tau)=\sum_{k=1}^{\infty} \tilde{p}_{k} \delta\left(\tau-\tau_{k}\right),
$$


where, $\tilde{p}_{k}$ is the power contained in the $k$ th bin. The total energy in the PDP of (6.6) is normalized such that $\sum_{k=1}^{\infty} \tilde{p}_{k}=1$. With this approach, $p_{k}$ (in $\mathrm{dB}$ ) for LOS case can be given as [144]

$$
p_{k}= \begin{cases}C & k=0, \\ Q+r-\beta \frac{\tau_{k}}{\bar{\tau}_{r m s}}+s_{k} & k>0,\end{cases}
$$

where, $Q$ is the normalizing factor that makes the sum of all $\tilde{p}_{k}$ 's equals to unity, $r$ is the difference between the power of second MPC and the power of first MPC which can be modeled as a Gaussian distribution with mean $\mu_{r}$ and variance $\sigma_{r}$, and $\beta$ is the power decay coefficient of the MPCs in the PDP. The RMS delay spread $\bar{\tau}_{r m s}$ is used as a normalizing factor, so that $\beta$ is dimensionless. The variable $s_{k}$ accounts for the random variations in PDP which can be modeled as a Gaussian distribution with zero mean and variance $\sigma_{s}$.

The random variable $C$ is the power of the first MPC in PDP that can be modeled as

$$
C=C_{0}-\gamma_{c} \log _{10}(d)+\epsilon_{c}
$$

where, $C_{0}$ is the average power of the first MPC at the reference distance $d_{0}, \gamma_{c}$ is a coefficient that accounts for the power decay of the first MPC with distance $d$, and $\epsilon_{c}$ is a zero mean Gaussian random variable with variance $\sigma_{c}$.

The power decay coefficient $\beta$ in (6.7) is modeled as

$$
\beta=\beta_{0}-\gamma \log _{10}(d)+\epsilon,
$$

where, $\beta_{0}$ is the average decay of $\mathrm{PDP}$ at the reference distance, $\gamma$ is a coefficient that accounts for the power decay of first MPC with distance $d$, and $\epsilon$ is a zero mean Gaussian random variable with variance $\sigma_{\epsilon}$. 


\subsection{Measurement and Simulation Results}

\subsubsection{CIR Measurements}

Using the channel sounding method described in Section 6.1, CIR measurements of a $100 \mathrm{MHz}$ channel were conducted in an indoor office environment for different line of sight (LOS) distances $(10 \mathrm{~m}-27 \mathrm{~m})$ between the transmitter and the receiver USRPs. For each distance, $K=100$ CIR measurements were recorded. Figure 6.3 shows the base-band equivalent CIR and frequency response measurements for a distance of $21 \mathrm{~m}$. Figure 6.4 shows the average PDP evaluated using the $100 \mathrm{CIR}$ measurements at $d=21 \mathrm{~m}$.

Using the measured CIRs, the calculated mean excess delay, RMS delay spread and coherence bandwidth of the channel are shown in Figure 6.5 as a function of distance. It can be observed that the RMS delay of channel increases with increase in the distance, causing the coherence bandwidth to decrease significantly.
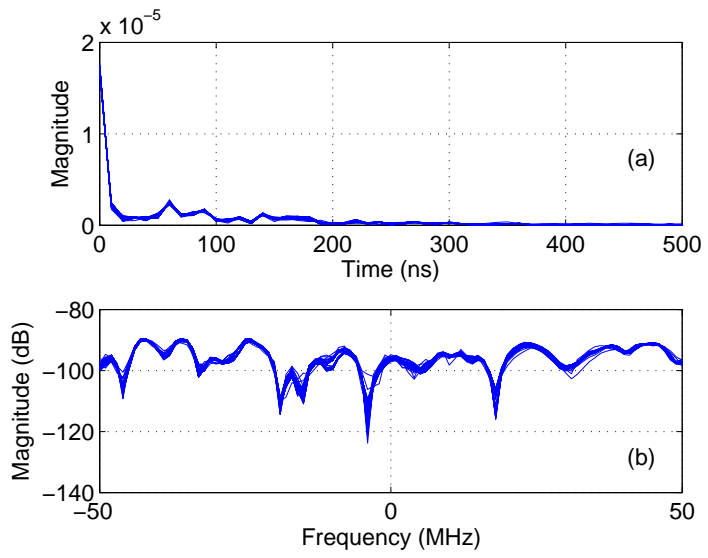

Figure 6.3: Overlaid measurements of base-band equivalent (a) channel impulse responses; and (b) channel frequency responses; at a distance $d=21 \mathrm{~m}$ from the transmitter.

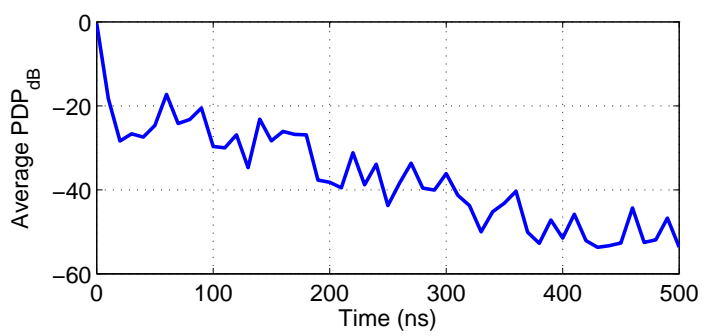

Figure 6.4: Average PDP of the channel for $d=21 \mathrm{~m}$. 


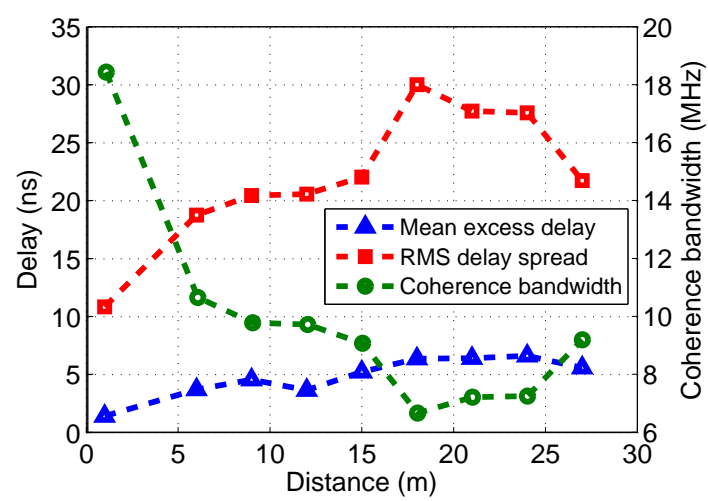

Figure 6.5: Mean excess delay, RMS delay spread and coherence bandwidth of the channel.

\subsubsection{Channel Modeling Parameters}

Figure 6.6 shows the measured average PL as a function of $10 \log _{10}(d)$ and the best line fit to the measured values. The best line fit was produced using (6.3) with $\alpha=1.66$ and $\overline{P L}_{\mathrm{dB}}\left(d_{0}\right)=43.42 \mathrm{~dB}$. The $\alpha$ is observed to be less than the free-space path loss exponent $\alpha_{\mathrm{FS}}=2$. This could be due to walls of the corridor being close to each other that may cause strong reflections and refractions, as also reported in [145] for LOS scenarios.

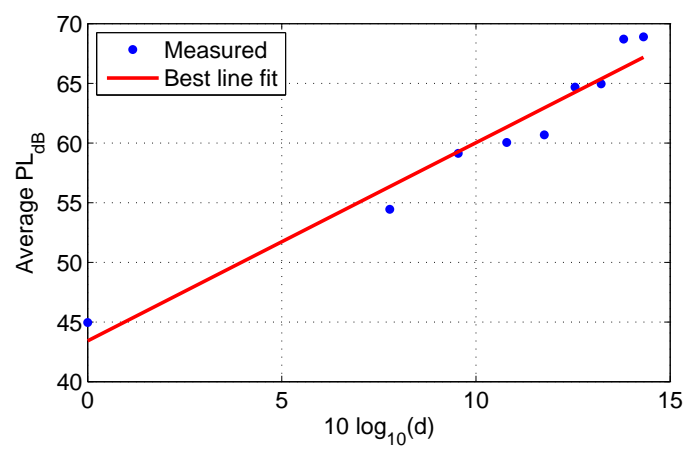

Figure 6.6: Measured average path loss and the best line fit for different distances.

The shadow fading model can be generated as described in Section 6.2.1 by finding the standard deviation of shadowing variable $X_{\sigma}$ that best fits the Gaussian distribution to the histogram of the measured samples that include all distances and CIR measurements. Figure 6.7a shows the histogram and the best fitting Gaussian distribution with $\sigma=0.117 \mathrm{~dB}$. This value of $\sigma$ is close to the value obtained in [146] for an indoor scenario with a LOS link between the transmitter and the receiver. 


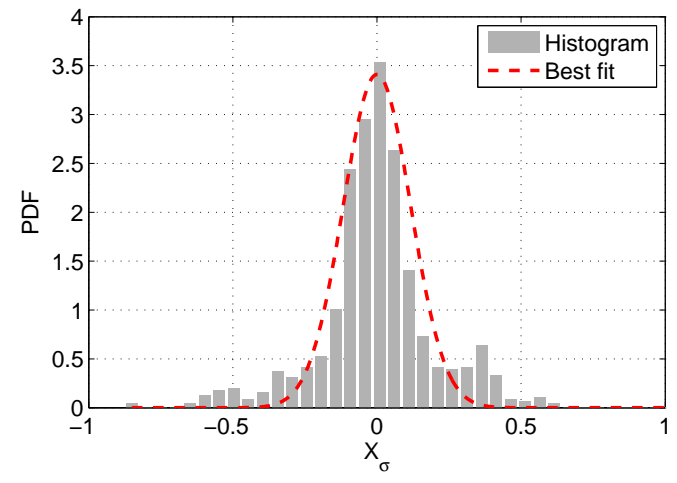

(a) Histogram of the shadowing variable $X_{\sigma}$.

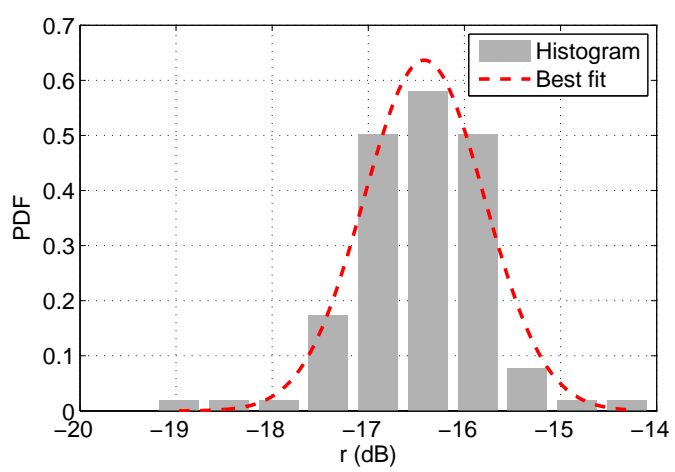

(b) Histogram of $r$.

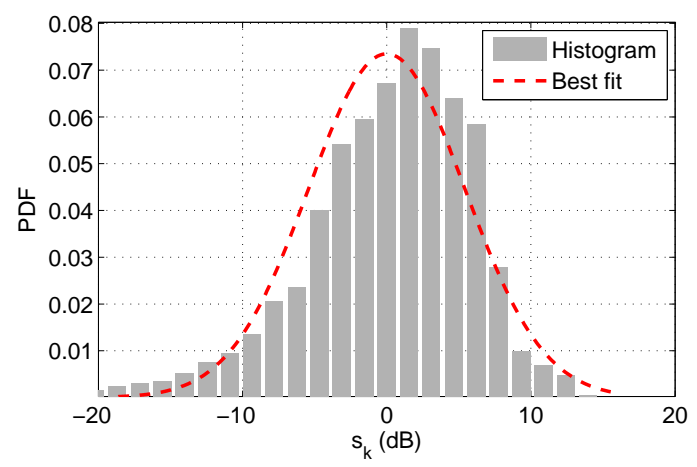

(c) Histogram of $s_{k}$.

Figure 6.7: Fitting of Gaussian distribution to the histograms of the channel parameters $X_{\sigma}, r$, and $s_{k}$.

Combining the path loss and shadowing models, the large scale fading can be expressed as,

$$
P L_{\mathrm{dB}}(d)=\overline{P L}_{\mathrm{dB}}\left(d_{0}\right)+16.6 \log _{10}\left(d / d_{0}\right)+X_{\sigma}
$$

With reference to (6.7), the histogram of $r$ which represents the difference between the power of second MPC and the power of first MPC is shown in Figure 6.7b. The best fitting Gaussian distribution is with the mean $\mu_{r}=-16.47 \mathrm{~dB}$ and standard distribution $\sigma_{r}=0.38 \mathrm{~dB}$. For $s_{k}$ in (6.7), the histogram and the best fitting Gaussian distribution with $\sigma_{s}=29.48 \mathrm{~dB}$ are shown in Figure 6.7c. Similarly, other parameters of the channel model are found through regressions using (6.8) and (6.9), and Table 6.1 shows the extracted values of all the parameters. Using these parameters, our channel model is validated in 
Figure 6.8, where the simulated PDP samples are compared with the measured PDP samples for different distances.
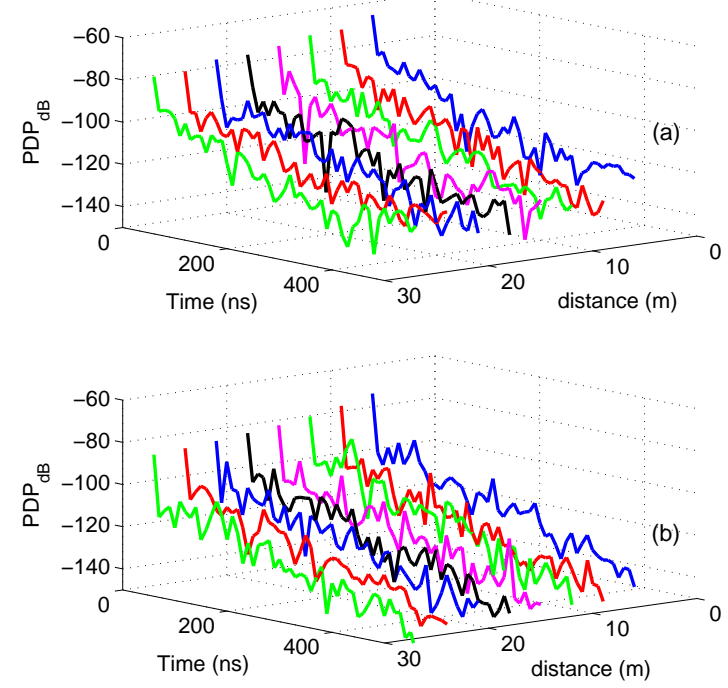

Figure 6.8: Comparison of the (a) measured, and (b) simulated PDP samples for different distances.

\subsubsection{Multi-hop versus Single-hop Performance}

The simulation setup for multi-hop communication is illustrated in Figure 6.9. The devices use OFDM to communicate over the channel, and the channel is modeled using the parameters summarized in Table 6.1. The transmitter can communicate with the receiver over a relay node with two-hops, or directly with a single-hop by transmitting with higher transmission power. By using transmit power control, the received powers at the relay and receiver nodes are assumed to be identical for both single-hop and two-hop cases. On the other hand, the channel selectivity characteristics of single and two-hop scenarios will be different due to different communication distances. Our goal is to investigate the system-level impact of the channel selectivity characteristics on single-hop and multi-hop communications.

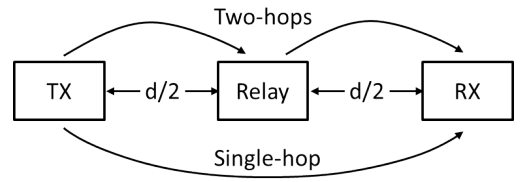

Figure 6.9: Multi-hop communication. 
Table 6.1: Parameters of channel model

\begin{tabular}{lc}
\hline Parameter & Value \\
\hline$\alpha$ & 1.66 \\
$\sigma$ & $0.12 \mathrm{~dB}$ \\
$\overline{P L}_{\mathrm{dB}}\left(d_{0}\right)$ & $43.42 \mathrm{~dB}$ \\
$C_{0}$ & $-56.62 \mathrm{~dB}$ \\
$\gamma_{c}$ & 21.91 \\
$\sigma_{c}$ & 0.026 \\
$\beta_{0}$ & -0.64 \\
$\gamma$ & 0.90 \\
$\sigma_{\epsilon}$ & 0.0052 \\
$\sigma_{s}$ & $29.48 \mathrm{~dB}$ \\
$\mu_{r}$ & $-16.47 \mathrm{~dB}$ \\
$\sigma_{r}$ & $0.38 \mathrm{~dB}$ \\
\hline
\end{tabular}

Simulations are carried out for three different distances $(50,75,100 \mathrm{~m})$ between the TX and RX in Figure 6.9, each with three different subcarrier frequency spacings $(60,80,100$ $\mathrm{kHz}$ ). The number of subcarriers is fixed to 64 , fifty two of which are the active subcarriers. With this simulation setup, we try to analyze how the distance and the OFDM subcarrier spacing affect the performance of multi-hop and single-hop communications.

The results that capture the detrimental effects of higher frequency selectivity in an OFDM system for the TX-RX link are shown in Figure 6.10. From this figure, we can clearly see that multi-hop communication yields a lower BER for a channel with lower frequency selectivity. Accordingly, the BER difference between the single-hop and two-hop cases increases with increasing subcarrier frequency spacing. Nonetheless, Figure 6.10 shows that multi-hop communication reduces the BER for all frequency spacing.

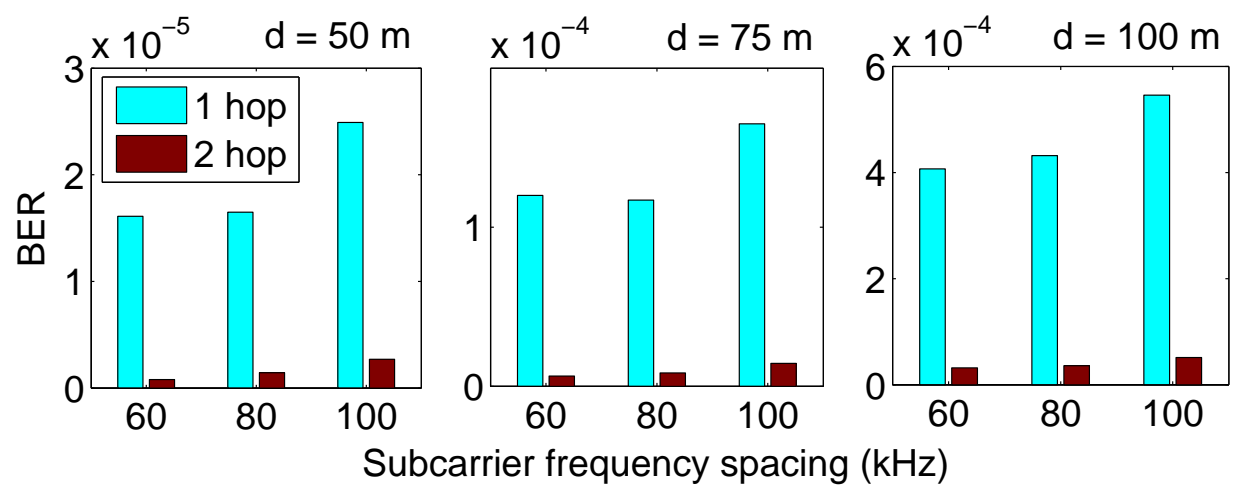

Figure 6.10: BER for different distances and subcarrier frequency spacings. 


\section{CHAPTER VII}

\section{Handover-Count based Velocity Estimation and Mobility State Detection}

Mobility management in cellular networks is an important task that is critical to provide good quality of service to the mobile users by minimizing the handover failures. Steps of a typical handover process in a HetNet scenario is illustrated in Figure 7.1. In order to initiate a handover, a UE waits until the received signal strength from a serving cell (macrocell) $P_{\mathrm{m}}$ is larger than the received signal strength from a target cell (picocell) $P_{\mathrm{p}}$ plus a hysterisis threshold (step-1). Even when this condition is satisfied, a UE waits for a duration of time-to-trigger (TTT), before sending the measurement reports to its serving cell (step-2). Use of a TTT is critical to ensure that ping-pongs are minimized due to fluctuations in the link qualities from different cells. If the condition in step-1 is still satisfied after the TTT, the UE sends the measurement reports to its serving BS in its uplink (step-3), which finalizes the handover by sending a handover command to the UE in the downlink (step-4). In homogeneous networks, the large size of the cells allows steps 1-4 in the handover process to be completed near the cell edges. Since the UE is connected to the serving BS through steps 1-4, high interference received from a target BS may frequently cause handover failure problems in HetNet scenarios, especially to the high mobility users [11,14]. A high speed UE moving into a small cell may reach deep inside the coverage area of the small cell before the handover can be finalized. These challenges in HetNets motivate the need for user and cell specific handover parameter optimization techniques for high speed users. Since the handover parameter optimization techniques are highly dependent on the velocity of a UE, estimating the UE's velocity is important for effective application of such optimization techniques.

A simple and efficient way to estimate a UE's velocity is by counting the number of handovers made by the UE during a predefined time window. Indeed, handover-count based mobility state detection has been standardized since LTE Release- 8 specifications. The increasing density of small cells in wireless networks can help in accurate estimation 


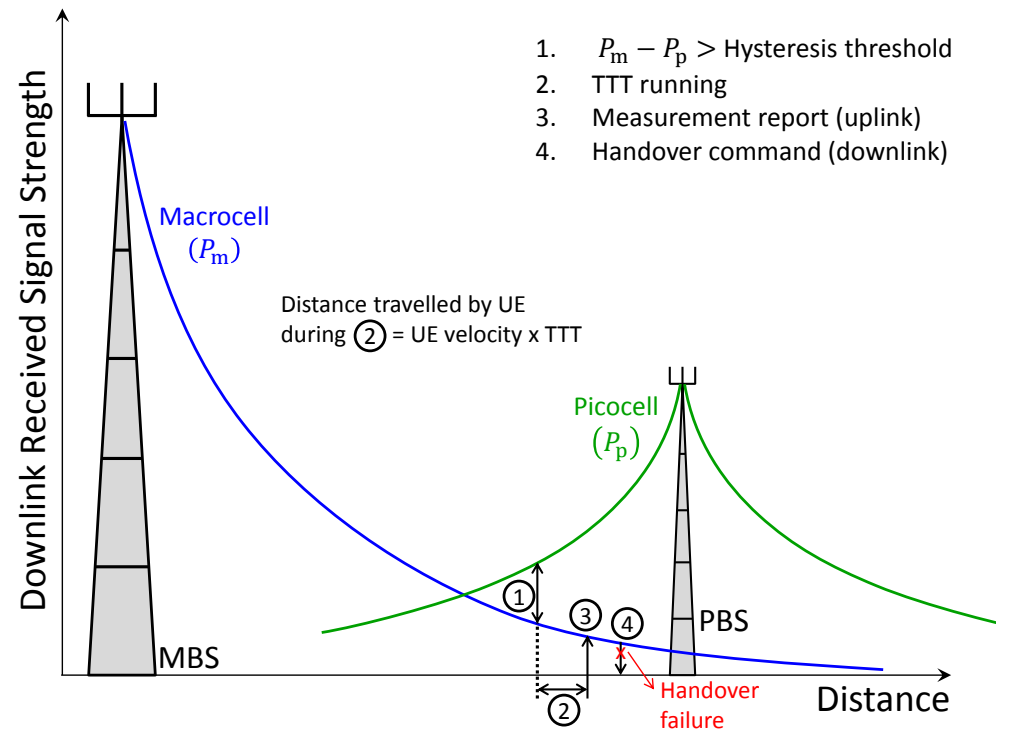

Figure 7.1: Handover failure problem in HetNets due to small cell size.

of velocity and mobility state of a UE. In this chapter, we introduce a novel and efficient handover-count $t^{1}$ based UE velocity estimation technique using the tools from stochastic geometry, and characterize its accuracy through CRLBs, when the density of SBSs is known. Since the service provider has the information of the number of SBSs in a particular geographic area, the SBS density in that area can be calculated and broadcasted as part of system information in next generation networks. The SBS density may also be signaled in a user-specific manner to the next generation UEs which are capable of velocity estimation.

Our contributions in this chapter are as follows:

- A densely deployed small cell network is modeled using stochastic geometry. For a given small-cell density, two approximations to the probability mass function (PMF) of handover-count of a UE are derived using a heuristic approach.

- Using the PMF approximations, expressions for the CRLB of velocity estimation are derived.

\footnotetext{
${ }^{1}$ Subsequently, "handover-count" is used in a broad sense to refer to the number of cells traversed by a UE. E.g., in phantom cells [147], a UE is always connected to the MBSs, and can get additional throughput from SBSs, minimizing handover failures.
} 
- A minimum variance unbiased (MVU) velocity estimator is derived whose variance tightly matches with the CRLB, and accuracy of the estimator is investigated for various UE speeds, SBS densities, and handover-count measurement times.

- The estimated velocity is used to detect the mobility state (low/medium/high) of a UE, and the expressions for the probability of detection and probability of false alarm are derived.

- Accuracy of the velocity estimator is analyzed for different realistic scenarios such as: RWP mobility model for the UEs, clustered deployment of SBSs, and variable UE velocity.

\section{$7.1 \quad$ System Model}

Consider a HetNet scenario where the MBSs tier and the SBSs tier use different frequency bands, as in phantom cell architecture [147]. We assume that the SBSs are randomly distributed according to a homogeneous PPP with intensity $\lambda$. As the SBSs use a dedicated frequency band which is different from the frequency band used by the MBSs, the coverage of a small cell would depend on the neighboring SBSs, but not on MBSs. Hence, the small cells of the network can be modeled using Poisson-Voronoi tessellation as illustrated in Figure 7.2(b). In Figure 7.2(a), co-channel deployment of small cells is illustrated in which the coverage of small cells is determined by the interference from neighboring SBSs and MBSs. We consider only the dedicated channel deployment of small cells as illustrated in Figure 7.2(b).

We consider a simple UE mobility scenario as also shown in Figure 7.2(b), in which the UE travels along a linear trajectory (for example, through $\mathrm{X}$-axis) with constant velocity $v$. During the travel, we assume that the UE can determine whenever it crosses the boundary of a small cell. In a broad sense, we call the boundary crossings made by the UE as handovers. Therefore, the number of handovers $H$ made by the UE during a measurement time window $T$ is equal to the number of intersections between the UE travel trajectory (of length $d=v T$ ) and the small-cell boundaries. We use linear mobility model for its simplicity in theoretical 


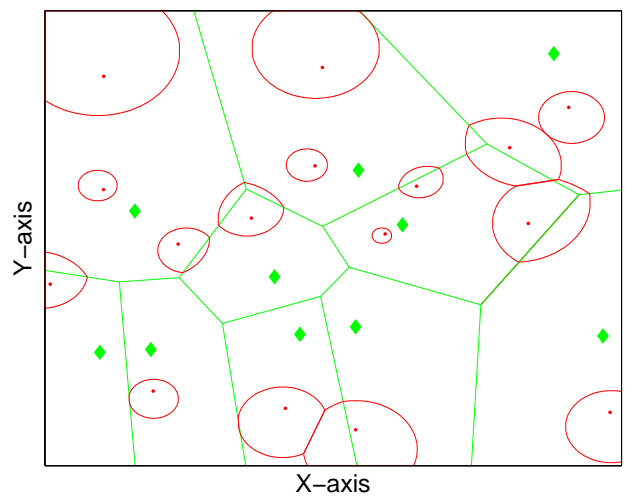

(a) Coverage of macrocells and small cells in co- (b) Coverage of small-cells with dedicated channel channel deployment, as in a typical HetNet.

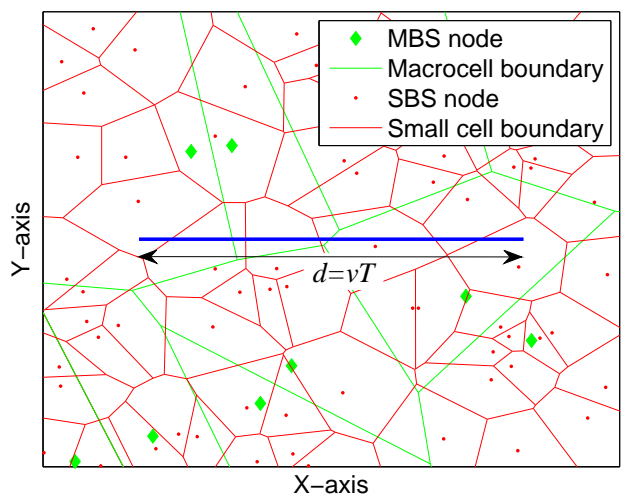

deployment as in a phantom cell network.

Figure 7.2: Illustration of cellular network layout.

analysis, and this model is suitable for scenarios such as medium/high speed cars and trains that may travel through downtown areas. There may be many small cells deployed in such urban areas in the future which may be referred to as ultra/hyper-dense networks [148]. Linear mobility may not be accurate for some other scenarios, and therefore we have also considered RWP mobility model which is more general and includes linear mobility as a special case.

Note that the approach in Figure 7.2(b) for handover-count based MSD of a UE, into low/medium/high mobility states, has already been specified in the LTE Release- 8 standard. Remarkably, no studies exist in the literature that investigate fundamental bounds and effective estimators for UE velocity. At high UE speeds and high SBS densities, handover failure of a UE becomes more likely $[13,149,150]$. Hence, the velocity estimation of UE based on handover counts may not work effectively with conventional LTE mobility management. On the other hand, emerging small-cell architectures such as phantom cells $[147,151]$ decouple the control and user planes, and allow the UE to be connected to macrocell all the time. Small-cells are discovered through special discovery signals [152], and a UE can connect them (reminiscent to a handover) to have higher throughput. Hence, handover-count based velocity estimation is still applicable in such scenarios. 

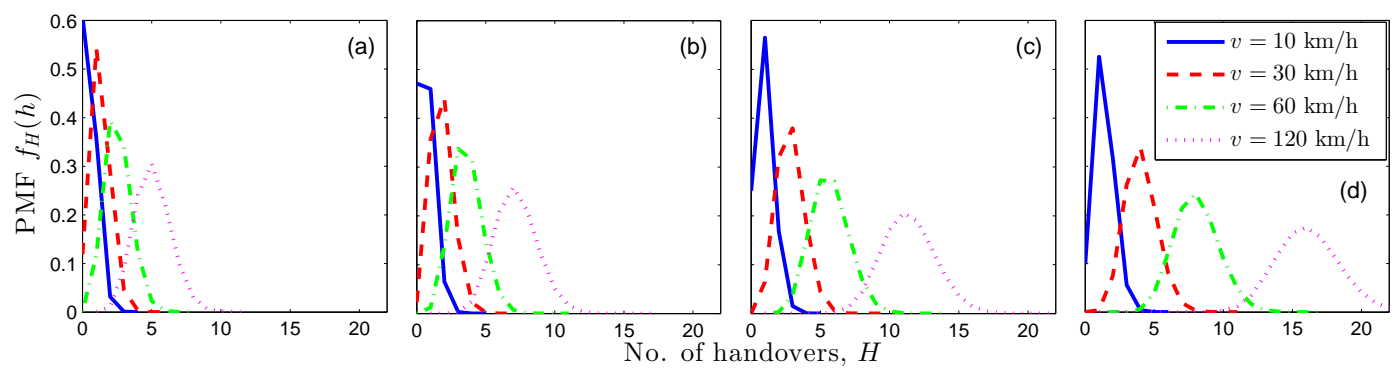

Figure 7.3: The PMF of the handover count for different $\lambda$ and $v$ values; (a) $\lambda=$ $100 \mathrm{SBSs} / \mathrm{km}^{2}$; (b) $\lambda=200 \mathrm{SBSs} / \mathrm{km}^{2}$; (c) $\lambda=500 \mathrm{SBSs} / \mathrm{km}^{2}$; (d) $\lambda=1000 \mathrm{SBSs} / \mathrm{km}^{2}$.

The handover count $H$ for a scenario as in Figure 7.2(b) is a discrete random variable and its statistics do not change with the direction of the linear trajectory because the SBS locations are modeled using a homogeneous and stationary PPP. The probability mass function $(\mathrm{PMF}) f_{H}(h)$ of handover count obtained through the simulations is shown in Figure 7.3(a)-7.3(d) for different SBS density $\lambda$ and UE velocity $v$ settings, with the handover-count measurement time fixed to $T=12 \mathrm{~s}$. For low SBS densities, as in Figure 7.3(a), the PMFs for different UE velocities are overlapping significantly, leading to low velocity estimation accuracies. For higher SBS densities, as in Figure 7.3(d), the PMFs for different velocities are separated, leading to better estimation accuracies. It should also be noticed that for higher UE velocities, standard deviation of the handover count also increases, implying lower estimation accuracies. Another characteristic of the PMF is that for large $\lambda$ and $v$ values, the shape of PMF resembles Gaussian distribution.

\subsubsection{Modeling Handover-Count Statistics}

In order to obtain the velocity estimate of a UE based on its handover count $H$, we need to know the PMF $f_{H}(h)$ of handover count. For a scenario as in Figure 7.2(b), an exact expression for the mean number of handovers can be derived as $[89,153]$

$$
E[H]=\frac{4 v T \sqrt{\lambda}}{\pi}
$$

To the best of our knowledge, there is no expression available for the PMF of handover count in the literature. Deriving an expression for the PMF $f_{H}(h)$ is a complicated and laborious 
task, which might result into a mathematically intractable expression [153]. Hence, we derive an approximation to the $\mathrm{PMF} f_{H}(h)$.

There are several papers in the literature where the PPP based parameters are approximated rather than deriving the exact expressions due to the complexity involved in the derivation of exact expressions. For example, in [154], geometrical characteristics of the perimeter, area and number of edges in a 2-dimensional Voronoi cell, and volume, surface area and number of faces in a 3-dimensional Voronoi cell are approximated by fitting generalized gamma distribution to the respective histograms. Similarly, the distributions of 2-dimensional cell area and 3-dimensional cell volume are approximated in [155] by fitting some simple expressions.

We derive two approximations to the $\mathrm{PMF} f_{H}(h)$ for the handover count:

1. approximation $f_{H}^{\mathrm{g}}(h)$ derived using gamma distribution;

2. approximation $f_{H}^{\mathrm{n}}(h)$ derived using Gaussian distribution.

These two approximations to the handover count PMF will be discussed in more detail in Section III, and their accuracies will be further investigated and compared in Section VI.A.

\subsection{Approximation of the Handover Count PMF Using Gamma and Gaussian Distributions}

In this section, two approximations for the PMF of handover count will be introduced.

In each approximation method, the parameters of a distribution (gamma distribution or Gaussian distribution) will be approximated using the curve fitting tools in Matlab.

\subsubsection{Approximation of the PMF of Handover Count using Gamma Distribu- tion}

Gamma distribution has been commonly used in approximating the statistical distribution of the parameters related to PPPs such as area, volume, number of edges, etc., of Poisson Voronoi cells $[154,155]$. It can be effectively used to approximate the handover count PMF. 
The gamma PDF can be expressed using the shape parameter $\alpha>0$ and rate parameter $\beta>0$ as

$$
f^{g}(x)=\frac{\beta^{\alpha}}{\Gamma(\alpha)} x^{\alpha-1} e^{-\beta x}, \text { for } x \in(0, \infty)
$$

where $\Gamma(\alpha)=\int_{0}^{\infty} t^{\alpha-1} e^{-t} \mathrm{~d} t$ is the gamma function. Fitting the gamma PDF to the PMF $f_{H}(h)$ is not a straight forward task since the gamma PDF is a continuous function while the PMF $f_{H}(h)$ is a discrete function. Therefore, we fit the subsets of gamma PDF to the PMF $f_{H}(h)$. The subsets of gamma PDF can be obtained by integrating the gamma PDF

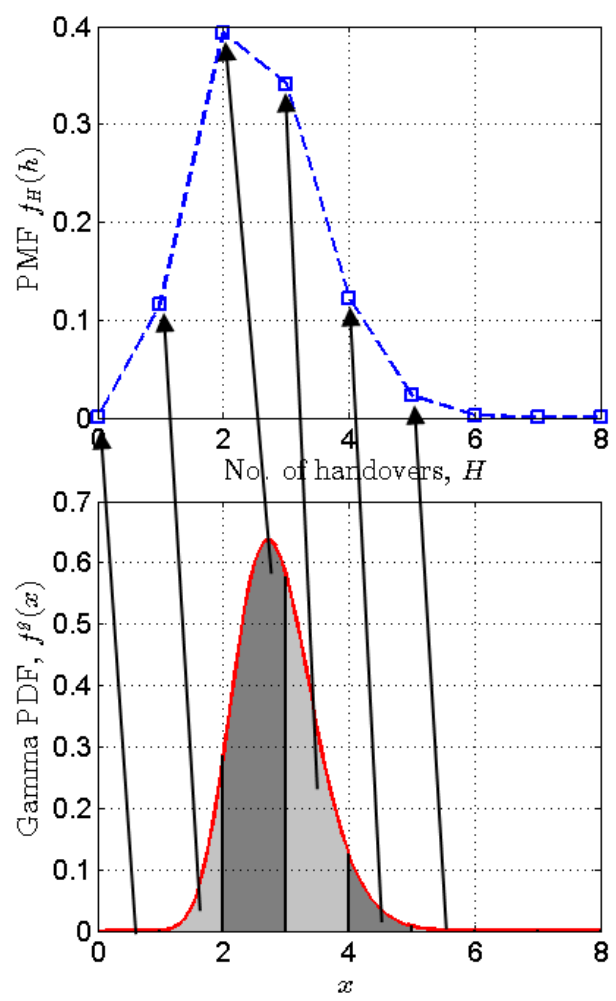

Figure 7.4: Illustration of fitting gamma distribution to the handover count PMF.

between the integer values of $x$ as illustrated in Figure 7.4. Integrating the gamma PDF between 0 and 1 provides the PMF value for $h=0$, integrating the gamma PDF between 1 and 2 provides the PMF value for $h=1$, and so on. This process can be mathematically 
described as:

$$
f_{H}^{\mathrm{g}}(h)=\int_{h}^{h+1} f^{\mathrm{g}}(x) \mathrm{d} x, \text { for } h \in\{0,1,2, \ldots\}
$$

where, $f_{H}^{\mathrm{g}}(h)$ is an approximation to the PMF $f_{H}(h)$. Substituting (7.2) into (7.3), we get

$$
f_{H}^{\mathrm{g}}(h)=\int_{h}^{h+1} \frac{\beta^{\alpha}}{\Gamma(\alpha)} x^{\alpha-1} e^{-\beta x} \mathrm{~d} x=\frac{\beta^{\alpha}}{\Gamma(\alpha)} \int_{h}^{h+1} x^{\alpha-1} e^{-\beta x} \mathrm{~d} x, \text { for } h \in\{0,1,2, \ldots\}
$$

With the change of variable $x=t / \beta$, we can rewrite (7.4) in its equivalent form as

$$
f_{H}^{\mathrm{g}}(h)=\frac{\beta^{\alpha}}{\Gamma(\alpha)} \int_{\beta h}^{\beta(h+1)}\left(\frac{t}{\beta}\right)^{\alpha-1} e^{-t} \frac{\mathrm{d} t}{\beta}=\frac{1}{\Gamma(\alpha)} \int_{\beta h}^{\beta(h+1)} t^{\alpha-1} e^{-t} \mathrm{~d} t=\frac{\Gamma(\alpha, \beta h, \beta(h+1))}{\Gamma(\alpha)},
$$

where, $\Gamma(\alpha, \beta h, \beta(h+1))=\int_{\beta h}^{\beta(h+1)} t^{\alpha-1} e^{-t} \mathrm{~d} t$ is the generalized incomplete gamma function. However, for the approximation in (7.5) to be accurate, the values for $\alpha$ and $\beta$ parameters should be chosen such that the mean squared error (MSE) between $f_{H}(h)$ and $f_{H}^{\mathrm{g}}(h)$ is minimized.

Lemma 1. The $\alpha$ and $\beta$ parameters for the approximation in (7.5) which minimize the MSE between $f_{H}(h)$ and $f_{H}^{\mathrm{g}}(h)$ can be expressed as

$$
\begin{aligned}
& \alpha=2.7+4 d \sqrt{\lambda}, \\
& \beta=\pi+\frac{0.8}{0.38+d \sqrt{\lambda}},
\end{aligned}
$$

where $d=v T$ is the distance traveled by UE during the handover-count measurement time.

Proof. See Appendix 3.

Using (7.5)-(7.7), we can capture the statistical distribution of handover count if the SBS density and the distance traveled by UE are known. Through (7.6) and (7.7), it can also be noticed that the handover-count distribution depends on the distance traveled by UE, rather than the UE velocity or the time period independently. 


\subsubsection{Approximation of the PMF of Handover Count using Gaussian Distri- bution}

In the previous section, approximation to the handover count PMF $f_{H}(h)$ was derived using gamma distribution which resulted into an expression in integral form. In this section, we

will approximate the PMF $f_{H}(h)$ using Gaussian distribution which results into a closed form expression for the handover-count PMF.

The PDF of Gaussian distribution can be expressed as a function of mean $\mu$ and variance $\sigma^{2}$ :

$$
f^{\mathrm{n}}(x)=\frac{1}{\sqrt{2 \pi \sigma^{2}}} e^{-\frac{(x-\mu)^{2}}{2 \sigma^{2}}}
$$

Since the PMF $f_{H}(h)$ is discrete and the Gaussian distribution in (7.8) is continuous, we consider only non-negative integer samples of the Gaussian distribution for the fitting process. Henceforth, the approximation to $f_{H}(h)$ can be expressed as,

$$
f_{H}^{\mathrm{n}}(h)=\frac{1}{\sqrt{2 \pi \sigma^{2}}} e^{-\frac{(h-\mu)^{2}}{2 \sigma^{2}}}, \text { for } h \in\{0,1,2, \ldots\}
$$

The values of $\mu$ and $\sigma^{2}$ should be chosen to minimize the MSE between $f_{H}(h)$ and $f_{H}^{\mathrm{n}}(h)$.

Lemma 2. The $\mu$ and $\sigma^{2}$ parameters for the approximation in (7.9) which minimize the MSE between $f_{H}(h)$ and $f_{H}^{\mathrm{n}}(h)$ can be expressed as

$$
\begin{aligned}
\mu & =\frac{4 d \sqrt{\lambda}}{\pi}, \\
\sigma^{2} & =0.07+0.41 d \sqrt{\lambda},
\end{aligned}
$$

where $d=v T$ is the distance traveled by the UE during the handover count measurement time.

Proof. See Appendix 4. 


\subsection{Cramer-Rao Lower Bound for Velocity Estimation}

CRLB can be used to serve as a lower bound on the variance of an unbiased estimator [156]. An estimator is said to be unbiased if its expected value is same as the true value of the parameter being estimated. An unbiased estimator whose variance can achieve the CRLB is said to be an efficient estimator, and it can achieve minimum MSE among all the unbiased estimators. In some scenarios, it might not be feasible to determine an efficient estimator. In that case, the unbiased estimator with the smallest variance is said to be a MVU estimator.

In this section, we will derive the CRLB for velocity estimation using the mathematical tools from estimation theory. Since we have two different approximations for the PMF of the number of handovers, we will obtain two separate CRLB expressions.

\subsubsection{CRLB Derivation using Gamma PMF Approximation}

In this sub-section we will obtain the CRLB by considering the handover count PMF approximation that was derived using gamma distribution in Section 7.2.1.

Theorem 1. In a Poisson-Voronoi tessellation of small cells with SBS node density $\lambda$, let a UE travel with velocity $v$ over a linear trajectory and make $H$ handovers over a time duration T. If the PMF of the handover count can be expressed using $f_{H}^{\mathrm{g}}(H ; v)$ as in $(7.5)$, then the CRLB for velocity estimation is given by

$$
\operatorname{var}(\hat{v}) \geq \frac{1}{\mathbb{E}\left[\left(\frac{\partial \log f_{H}^{\mathrm{g}}(H ; v)}{\partial v}\right)^{2}\right]}
$$


where, $\mathbb{E}[\cdot]$ is the expectation operator with respect to $H$, and

$$
\begin{aligned}
\frac{\partial \log f_{H}^{\mathrm{g}}(h ; v)}{\partial v}= & \frac{4 T \sqrt{\lambda} \beta^{\alpha}}{\alpha^{2} \Gamma(\alpha, \beta h, \beta(h+1))}\left[h_{2}^{\alpha} F_{2}(\alpha, \alpha ; \alpha+1, \alpha+1 ;-\beta h)\right. \\
& \left.\quad-(h+1)^{\alpha}{ }_{2} F_{2}(\alpha, \alpha ; \alpha+1, \alpha+1 ;-\beta(h+1))\right] \\
& -\frac{4 T \sqrt{\lambda}}{\Gamma(\alpha, \beta h, \beta(h+1))}[\gamma(\alpha, \beta h) \log (\beta h)-\gamma(\alpha, \beta(h+1)) \log (\beta(h+1))] \\
& +\frac{0.8 T \sqrt{\lambda} \beta^{\alpha-1} e^{-\beta h}\left[h^{\alpha}-e^{-\beta}(h+1)^{\alpha}\right]}{\Gamma(\alpha, \beta h, \beta(h+1))(0.38+v T \sqrt{\lambda})^{2}}-4 T \sqrt{\lambda} \psi(\alpha),
\end{aligned}
$$

where, $\psi(\cdot)$ is digamma function, $\gamma(\alpha, x)=\int_{0}^{x} t^{\alpha-1} e^{-t} \mathrm{~d} t$ is lower incomplete gamma function, and ${ }_{2} F_{2}\left(a_{1}, a_{2} ; b_{1}, b_{2} ; z\right)$ is generalized hypergeometric function which can be expressed as

$$
{ }_{2} F_{2}\left(a_{1}, a_{2} ; b_{1}, b_{2} ; z\right)=\sum_{k=0}^{\infty} \frac{\left(a_{1}\right)_{k}\left(a_{2}\right)_{k}}{\left(b_{1}\right)_{k}\left(b_{2}\right)_{k}} \frac{z^{k}}{k !},
$$

where, $(a)_{0}=1$ and $(a)_{k}=a(a+1)(a+2) \ldots(a+k-1)$, for $k \geq 1$.

Proof. See Appendix 5.

Due to the complexity of expression in (7.13), it is impractical to derive the right hand side (RHS) of (7.12) in closed form. For this reason, we can only find asymptotic CRLB by numerically evaluating the RHS of (7.12). Through simulations, we generate $N$ samples of the random variable $H$ and denote them as $\left\{H_{n}\right\}$, for $n \in 1,2, \ldots, N$. Using these $N$ samples, we can numerically evaluate the asymptotic CRLB using

$$
\operatorname{var}(\hat{v}) \geq \frac{N}{\sum_{m=H_{\min }}^{H_{\max }}\left(N_{m}\left(\frac{\partial \log f_{H}^{\mathrm{g}}(m ; v)}{\partial v}\right)^{2}\right)}
$$

where, $H_{\max }=\max \left\{H_{n}: \forall n \in 1,2, \ldots, N\right\}$, is the maximum value of $H_{n}, H_{\min }=\min \left\{H_{n}\right.$ : $\forall n \in 1,2, \ldots, N\}$, is the minimum value of $H_{n}$, and $N_{m}=\sum_{n=1}^{N} 1\left\{H_{n}=m\right\}$ is the number of elements in the set $\left\{H_{n}\right\}$ that are equal to $m$. Here, $1\{\cdot\}$ is the indicator function whose value is 1 if the condition inside the braces is true, 0 otherwise. 


\subsubsection{CRLB Derivation using Gaussian PMF Approximation}

In this sub-section, we will obtain the CRLB by considering the PMF approximation using Gaussian distribution that was derived in Section 7.2.2.

Theorem 2. In a Poisson-Voronoi tessellation of small cells with SBS node density $\lambda$, let a UE travel with velocity $v$ over a linear trajectory and make $H$ handovers over a time duration T. If the PMF of the handover count can be expressed using $f_{H}^{\mathrm{n}}(H ; v)$ as in $(7.9)$, then the CRLB for velocity estimation is given by

$$
\operatorname{var}(\hat{v}) \geq \frac{1}{\left(\frac{\mu}{v \sigma}\right)^{2}+\frac{1}{2}\left(\frac{0.41 T \sqrt{\lambda}}{\sigma^{2}}\right)^{2}}
$$

Proof. Consider the PMF approximation $f_{H}^{\mathrm{n}}(h)$ in $(7.9)$ which can be represented as a general Gaussian distribution, $H \sim \mathcal{N}\left(\mu, \sigma^{2}\right)$, where $\mu$ and $\sigma^{2}$ are given by (7.10) and (7.11) respectively. The Fisher information for the general Gaussian observations is given by $[156$, Section 3.9$]$

$$
I(v)=\left(\frac{\partial \mu}{\partial v}\right)^{2} \frac{1}{\sigma^{2}}+\frac{1}{2\left(\sigma^{2}\right)^{2}}\left(\frac{\partial \sigma^{2}}{\partial v}\right)^{2}=\left(\frac{4 T \sqrt{\lambda}}{\pi}\right)^{2} \frac{1}{\sigma^{2}}+\frac{1}{2\left(\sigma^{2}\right)^{2}}(0.41 T \sqrt{\lambda})^{2}
$$

Using inverse of the Fisher information, the CRLB can be expressed as in (7.16).

\subsubsection{Minimum Variance Unbiased Estimator for UE Velocity}

In Section 7.3.1 and Section 7.3.2, two CRLB expressions were derived by considering gamma and Gaussian distributions, respectively, for approximating the handover count PMF. In the case of using gamma distribution, the CRLB expression was complicated and not in closed form. On the other hand, in the case of using Gaussian distribution, the CRLB expression was relatively simple and in closed form. Hence, in this sub-section, we will consider the case with Gaussian distribution and derive an estimator $\hat{v}$ for a UE's velocity, which takes the number of handovers $H$ as the input. We will further derive the mean and the variance of this estimator and show that it is a MVU estimator. 
To derive the MVU velocity estimator, we first use Neyman-Fisher factorization to find the sufficient statistic for $v[156$, Section 5.4]. Then, we make use of Rao-BlackwellLehmann-Scheffe (RBLS) theorem to find the MVUE [156, Section 5.5]. The Neyman-Fisher factorization theorem states that if we can factor the PMF $f_{H}^{\mathrm{n}}(h)$ as

$$
f_{H}^{\mathrm{n}}(h)=g(\mathcal{F}(h), v) r(h),
$$

where $g$ is a function depending on $h$ only through $\mathcal{F}(h)$ and $r$ is a function depending only on $h$, then $\mathcal{F}(h)$ is a sufficient statistic for $v$. Using (7.9) and letting $\mathcal{F}(h)=h$, we can factor the $\mathrm{PMF} f_{H}^{\mathrm{n}}(h)$ in the form of $(7.18)$ as

$$
\begin{aligned}
& f_{H}^{\mathrm{n}}(h)=\underbrace{\frac{1}{\sqrt{2 \pi \sigma^{2}}} e^{-\frac{(\mathcal{F}(h)-\mu)^{2}}{2 \sigma^{2}}}} \cdot \underbrace{1}, \\
& g(\mathcal{F}(h), v) \quad r(h)
\end{aligned}
$$

Therefore, the sufficient statistic for $v$ is $\mathcal{F}(h)=h$. The sufficient statistic can be used to find the MVU estimator by determining a function $s$ so that $\hat{v}=s(\mathcal{F})$ is an unbiased estimator of $v$. By inspecting the relationship between the mean number of handovers $\bar{H}$ and the velocity $v$ in (7.1), we can formulate an estimator for $v$ as:

$$
\hat{v}=\frac{\pi H}{4 T \sqrt{\lambda}}
$$

In order to evaluate whether this estimator is unbiased, the expectation of the above estimator can be derived as

$$
E[\hat{v}]=E\left[\frac{\pi H}{4 T \sqrt{\lambda}}\right]=\frac{\pi}{4 T \sqrt{\lambda}} E[H]=\frac{\pi}{4 T \sqrt{\lambda}} \mu .
$$

Plugging (7.10) into (7.21), we get

$$
E[\hat{v}]=\frac{\pi}{4 T \sqrt{\lambda}} \frac{4 v T \sqrt{\lambda}}{\pi}=v .
$$


Therefore, the estimator $\hat{v}$ expressed in (7.20) is unbiased. Since this estimator is derived through RBLS theorem, it is an MVU estimator. To determine whether it is an efficient estimator, we derive the variance of the MVU estimator as follows:

$$
\operatorname{var}(\hat{v})=\operatorname{var}\left(\frac{\pi H}{4 T \sqrt{\lambda}}\right)=\left(\frac{\pi}{4 T \sqrt{\lambda}}\right)^{2} \operatorname{var}(H)=\left(\frac{v \sigma}{\mu}\right)^{2}
$$

Comparing (7.23) with (7.16), we can notice that the variance of MVU estimator is greater than the CRLB, and hence, the derived estimator is not an efficient estimator. Nevertheless, in Section 7.5.3, we show that the variance of the MVU estimator is very close to the CRLB.

\subsection{Mobility State Detection}

In this section, we will perform statistical analysis of MSD, in which a UE is categorized into one of the three different mobility states: low mobility, medium mobility and high mobility, as in 3GPP LTE Release-8 specifications $[81,157,158]$. We assume that the unbiased velocity estimator derived in Section 7.3.3 is used to estimate the UE velocity, and we will derive expressions for the probabilities that a UE is categorized into each of the three mobility states.

Using the estimated UE velocity $\hat{v}$ from (7.20), the UE can be categorized into one of the three mobility states: low $\left(S_{\mathrm{L}}\right)$, medium $\left(S_{\mathrm{M}}\right)$, and high $\left(S_{\mathrm{H}}\right)$, based on the following conditions:

$$
\mathcal{S}= \begin{cases}S_{\mathrm{L}} & \text { if } \quad \hat{v} \leq v_{\mathrm{l}} \\ S_{\mathrm{M}} & \text { if } \quad v_{\mathrm{l}}<\hat{v} \leq v_{\mathrm{u}} \\ S_{\mathrm{H}} & \text { if } \quad \hat{v}>v_{\mathrm{u}}\end{cases}
$$

where, $\mathcal{S} \in\left\{S_{\mathrm{L}}, S_{\mathrm{M}}, S_{\mathrm{H}}\right\}$ is the detected mobility state of the UE. The thresholds $v_{\mathrm{l}}$ and $v_{\mathrm{u}}$ are the lower and upper velocity thresholds, respectively, based on which a UE is classified into one of the three mobility states. 


\subsubsection{Mobility State Probabilities}

For a given velocity $v$, we define mobility state probability as the probability that the UE is categorized into a particular state. We can define the following three mobility state probabilities:

$P\left(\mathcal{S}=S_{\mathrm{L}} ; v\right) \rightarrow$ Probability that the mobility state is detected as $S_{\mathrm{L}}$, for a velocity $v ;$

$P\left(\mathcal{S}=S_{\mathrm{M}} ; v\right) \rightarrow$ Probability that the mobility state is detected as $S_{\mathrm{M}}$, for a velocity $v ;$

$P\left(\mathcal{S}=S_{\mathrm{H}} ; v\right) \rightarrow$ Probability that the mobility state is detected as $S_{\mathrm{H}}$, for a velocity $v$.

For a given velocity $v$, as the number of handovers $H$ is a random variable, the velocity $\hat{v}$ estimated using (7.20) is also a random variable. Hence, there will be false alarms and missed detections for calculating the mobility state.

Next, we derive analytic expressions for the mobility state probabilities. Using (7.20), we can express the PMF of $\hat{v}$ as,

$$
f_{\hat{v}}(\nu)=P(\hat{v}=\nu)=P\left(\frac{\pi H}{4 T \sqrt{\lambda}}=\nu\right)=P\left(H=\frac{4 T \sqrt{\lambda} \nu}{\pi}\right)=f_{H}\left(\frac{4 T \sqrt{\lambda} \nu}{\pi}\right)=f_{H}(h)
$$

where, $h=\frac{4 T \sqrt{\lambda} \nu}{\pi}$. Using the approximation of PMF $f_{H}(h)$ with Gaussian distribution as in (7.9), we can approximate the PMF of $\hat{v}$ as,

$$
f_{\hat{v}}(\nu)=f_{H}(h) \approx f_{H}^{\mathrm{n}}(h)=\frac{1}{\sqrt{2 \pi \sigma^{2}}} e^{-\frac{(h-\mu)^{2}}{2 \sigma^{2}}}, \text { for } h \in\{0,1,2, \ldots\}
$$


Now, we can express the three mobility state probabilities as

$$
\begin{gathered}
P\left(\mathcal{S}=S_{\mathrm{L}} ; v\right)=P\left(\hat{v} \leq v_{\mathrm{l}}\right) \approx \sum_{h=0}^{h_{1}} f_{H}^{\mathrm{n}}(h), \\
P\left(\mathcal{S}=S_{\mathrm{M}} ; v\right)=P\left(v_{\mathrm{l}}<\hat{v} \leq v_{\mathrm{u}}\right) \approx \sum_{h=h_{\mathrm{l}}+1}^{h_{\mathrm{u}}} f_{H}^{\mathrm{n}}(h), \\
P\left(\mathcal{S}=S_{\mathrm{H}} ; v\right)=P\left(\hat{v}>v_{\mathrm{u}}\right) \approx \sum_{h=h_{\mathrm{u}}+1}^{\infty} f_{H}^{\mathrm{n}}(h), \\
\text { where, } \quad h_{\mathrm{l}}=\left\lfloor\frac{4 T \sqrt{\lambda} v_{\mathrm{l}}}{\pi}\right\rfloor, \quad \text { and } \quad h_{\mathrm{u}}=\left\lfloor\frac{4 T \sqrt{\lambda} v_{\mathrm{u}}}{\pi}\right\rfloor,
\end{gathered}
$$

are the optimum lower and upper handover count thresholds for MSD, respectively. Given the velocity thresholds $v_{\mathrm{l}}$ and $v_{\mathrm{u}}$, the choice of handover count thresholds has a direct impact on the probability of correctly detecting the mobility state of a UE based on its velocity [159]. In (7.30), we have theoretically derived the handover-count thresholds for MSD which are optimum for the given velocity thresholds. In other related works in the literature, the handover-count thresholds for MSD have been determined through simulations. In $[13$, $159,160]$, the handover-count thresholds for MSD are found heuristically by considering the cumulative distribution function (CDF) plots of handover counts, for few different UE velocities. In [161], the handover counts are assumed to be distributed as Gaussian PDF, and the optimum handover-count thresholds are obtained by using the handover-count PDFs for few different UE velocities. However, in these prior works, the optimum handovercount thresholds are determined for some particular values of BS density and measurement time windows. Moreover, the statistical relationship between the UE velocity and the handover count is not considered. In this chapter, we have derived general expressions for the optimum handover-count thresholds as a function of SBS density $\lambda$, handover-count measurement time $T$, and velocity thresholds $\left(v_{\mathrm{l}}\right.$ and $\left.v_{\mathrm{u}}\right)$. 


\subsubsection{Probability of Detection and Probability of False Alarm}

The probability of detection is the probability that the mobility state of a UE is detected correctly. Mathematically, it can be expressed as

$$
P_{\mathrm{D}}=\left\{\begin{array}{lll}
P\left(\mathcal{S}=S_{\mathrm{L}} ; v\right) & \text { if } \quad v \leq v_{\mathrm{l}}, \\
P\left(\mathcal{S}=S_{\mathrm{M}} ; v\right) & \text { if } \quad v_{\mathrm{l}}<v \leq v_{\mathrm{u}}, \\
P\left(\mathcal{S}=S_{\mathrm{H}} ; v\right) & \text { if } \quad v>v_{\mathrm{u}} .
\end{array}\right.
$$

The probability of false alarm is the probability that the mobility state is detected incorrectly, which can be mathematically expressed as $P_{\mathrm{FA}}=1-P_{\mathrm{D}}$.

Consider an illustrative example in which the UE velocity is $v=60 \mathrm{~km} / \mathrm{h}$, SBS density is $\lambda=1000 \mathrm{SBSs} / \mathrm{km}^{2}$, and handover-count measurement time is $T=12 \mathrm{~s}$. The PMFs $f_{H}(h)$ and $f_{\hat{v}}(\nu)$ are shown in Figs. 7.5(a) and 7.5(b), respectively, which were obtained through Monte Carlo Simulations. In Figure 7.5(b), the range of $\hat{v}$ is divided into three regions $S_{\mathrm{L}}, S_{\mathrm{M}}$ and $S_{\mathrm{H}}$ that are separated from each other through the velocity thresholds $v_{\mathrm{l}}=40 \mathrm{~km} / \mathrm{h}$ and $v_{\mathrm{u}}=80 \mathrm{~km} / \mathrm{h}$. It can be noticed in Figure $7.5(\mathrm{~b})$ that even though the actual UE velocity is a constant $v=60 \mathrm{~km} / \mathrm{h}$ which belongs to $S_{\mathrm{M}}$ state, the estimated velocity is spread into a range of velocities. Hence, there is a small probability that the mobility state could be erroneously detected as $S_{\mathrm{L}}$ or $S_{\mathrm{H}}$ states, which is the probability of false alarm $P_{\mathrm{FA}}$. On the other hand, majority of the times the mobility state would be correctly detected as $S_{\mathrm{M}}$, which is the probability of detection $P_{\mathrm{D}}$.

With the particular parameter settings shown in Figure 7.5, the probabilities of different mobility states can be evaluated using (7.27)-(7.29) as $P\left(\mathcal{S}=S_{\mathrm{L}} ; v=60\right)=0.047, \quad P(\mathcal{S}=$ $\left.S_{\mathrm{M}} ; v=60\right)=0.8821, \quad P\left(\mathcal{S}=S_{\mathrm{H}} ; v=60\right)=0.0709$, respectively. Then, the probabilities of detection and false alarm can be evaluated using $(7.31)$ as $P_{\mathrm{D}}=P\left(\mathcal{S}=S_{\mathrm{M}} ; v=60\right)=$ $0.8821, P_{\mathrm{FA}}=P\left(\mathcal{S}=S_{\mathrm{L}} ; v=60\right)+P\left(\mathcal{S}=S_{\mathrm{H}} ; v=60\right)=0.1179$, respectively. 

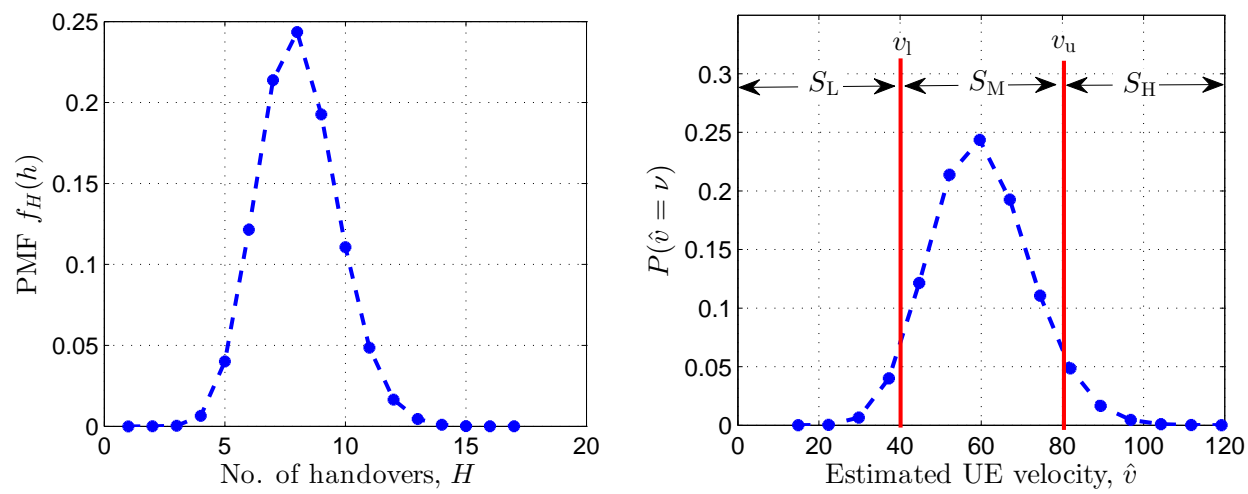

Figure 7.5: (a) PMF of the number of handovers; (b) PMF of the estimated velocity; $v=60 \mathrm{~km} / \mathrm{h}, \lambda=1000 \mathrm{SBSs} / \mathrm{km}^{2}, T=12 \mathrm{~s}, v_{\mathrm{l}}=40 \mathrm{~km} / \mathrm{h}$ and $v_{\mathrm{u}}=80 \mathrm{~km} / \mathrm{h}$.

\subsection{Numerical Results}

In this section, firstly, we will validate the accuracy of gamma PMF approximation $f_{H}^{\mathrm{g}}(h)$ and Gaussian PMF approximation $f_{H}^{\mathrm{n}}(h)$ by plotting their MSE performances. Secondly, we will plot the CRLBs and analyze the achievable accuracy of a UE's velocity estimate for different SBS density $\lambda$, UE velocity $v$, and handover count measurement time $T$. Finally, we will plot the variance of the MVU velocity estimator derived in Section 7.3.3 and show that it is approximately equal to the CRLB.

\subsubsection{Accuracy of PMF Approximation}

Approximations to the handover count PMF were derived using gamma distribution and Gaussian distribution in Sections 7.2.1 and 7.2.2, respectively. In this sub-section, we will quantify the accuracy of each approximation method by evaluating the MSE between the approximation and the PMF $f_{H}(h)$. The MSE can be expressed as

$$
M S E=\frac{1}{N_{\mathrm{h}}} \sum_{h=1}^{N_{\mathrm{h}}}\left[f_{H}^{u}(h)-f_{H}(h)\right]^{2}, \text { for } u \in\{\mathrm{g}, \mathrm{n}\},
$$

where $N_{\mathrm{h}}$ is the number of points in the PMF. The characteristics of MSE with respect to the variations in $\lambda$ and $v$ are shown in Figure 7.6 for the two approximation methods. In general, the MSEs of both approximation methods decrease with the increase in SBS 


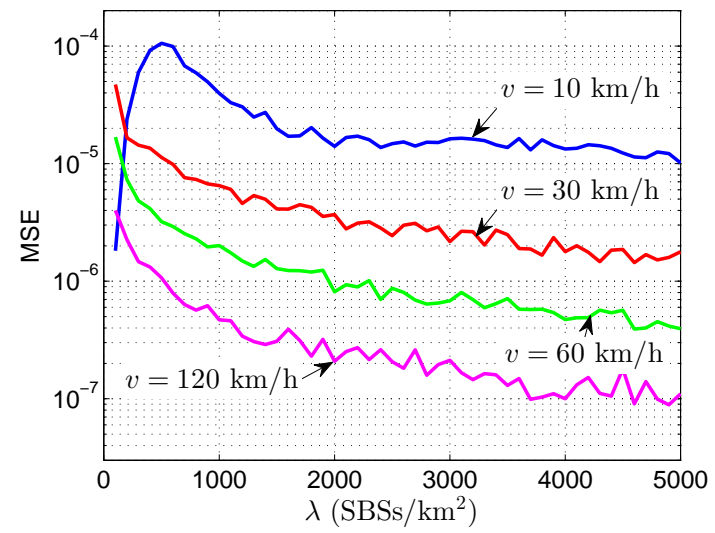

(a) PMF approximation using gamma distribution.

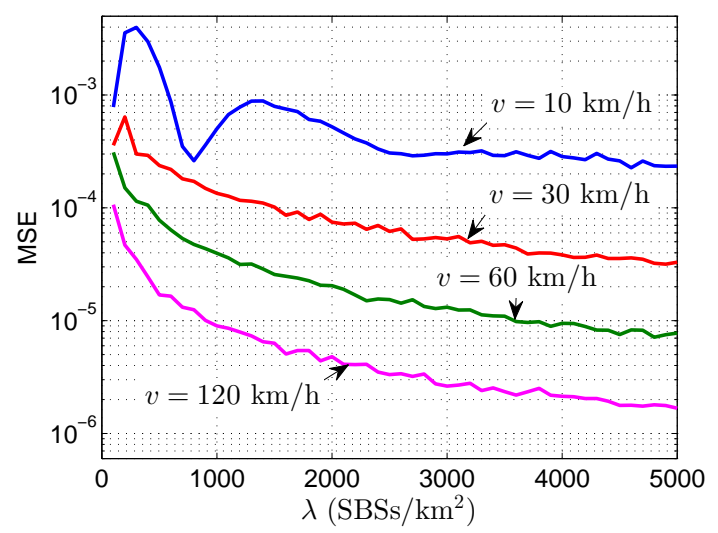

(b) PMF approximation using Gaussian distribution.

Figure 7.6: MSE versus $\lambda$ for different $v$, and $T=12 \mathrm{~s}$.

density $\lambda$ or UE velocity $v$. In other words, higher SBS density and higher UE velocity leads to better accuracy of the PMF approximation. By comparing Figure 7.6(a) and Figure 7.6(b), it can be noticed that the approximation using gamma distribution provides approximately ten times smaller MSE than the approximation using Gaussian distribution. However, the approximation using gamma distribution is not in closed form and hence it is more complicated than the approximation using Gaussian distribution. Therefore, there exists a trade-off between the accuracy and the complexity while making a choice between the two approximation approaches.

\subsubsection{CRLB Results}

The CRLB plots for UE velocity estimate $\hat{v}$ are shown in Figure 7.7 for the variations in UE velocity $v$ and SBS density $\lambda$. In the case of gamma approximation of $\operatorname{PMF} f_{H}(h)$, the CRLB plots in Figure 7.7(a) are obtained by numerically evaluating the expression in (7.15). On the other hand, the CRLB plots in Figure 7.7(b) are obtained using the closed form expression in (7.16), for the case of Gaussian approximation of PMF $f_{H}(h)$. It can be observed that the CRLB plots in Figure 7.7(a) and Figure 7.7(b) are similar and follow same trends with respect to $\lambda$ and $v$. However, we can expect that the plots in Figure 7.7(a) are more accurate because of the smaller MSE of gamma approximation method. On the other 


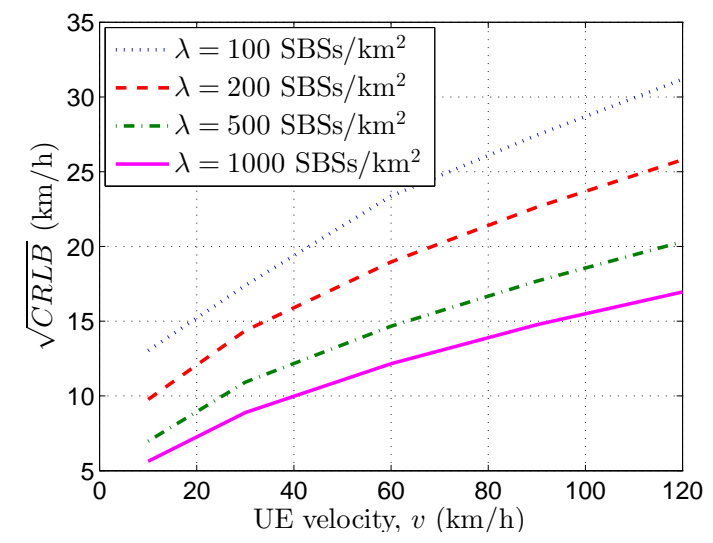

(a) CRLB derived using $f_{H}^{\mathrm{g}}(h)$.

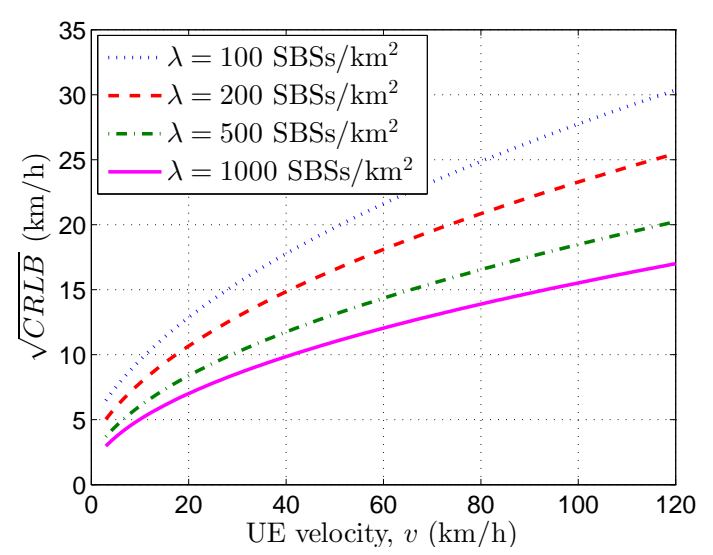

(b) CRLB derived using $f_{H}^{\mathrm{n}}(h)$.

Figure 7.7: CRLB versus $v$ for different $\lambda$, and $T=12 \mathrm{~s}$.

hand, the closed form CRLB expression in (7.16) for the case of Gaussian approximation can provide more insights, for example, on the dependence of CRLB into different parameters.

From Figure 7.7, it can be noticed that the CRLB increases with the increasing UE velocity $v$. This is because the variance of the number of handovers $H$ increases with increasing $v$, which can be observed in Figure 7.3. In contrast, the CRLB decreases with increasing SBS density $\lambda$, which can also be intuitively understood from Figure 7.3. With $\lambda=100 \mathrm{SBSs} / \mathrm{km}^{2}$, the peaks of the PMFs for different UE velocities are closely spaced with each other making it difficult to distinguish between the different UE velocities, resulting into higher CRLB. With $\lambda=1000 \mathrm{SBSs} / \mathrm{km}^{2}$, the peaks of the PMFs have more spacing between them making it easier to distinguish between the different UE velocities, resulting into lower CRLB. Results in Figure 7.7 show that for all considered SBS densities, using a handover count based approach, a UE's velocity can be estimated with a root mean squared error (RMSE) of $20 \mathrm{~km} / \mathrm{h}$ for UE velocities less than $40 \mathrm{~km} / \mathrm{h}$. For UE velocities on the order of $120 \mathrm{~km} / \mathrm{h}$, velocity can still be estimated with a RMSE less than $31 \mathrm{~km} / \mathrm{h}$ for densities of $100 \mathrm{SBSs} / \mathrm{km}^{2}$, which can be further improved for higher SBS densities.

In related works of the literature [81,159-161], a fixed measurement time interval of $30 \mathrm{~s}$, $60 \mathrm{~s}$, or $120 \mathrm{~s}$ is used for the MSD of UEs. However, we have used a smaller measurement time interval of $T=12 \mathrm{~s}$ in Figure 7.7 so that the velocity estimator can provide quicker results. At the same time, $T=12 \mathrm{~s}$ also provides a reasonable estimation accuracy. For 


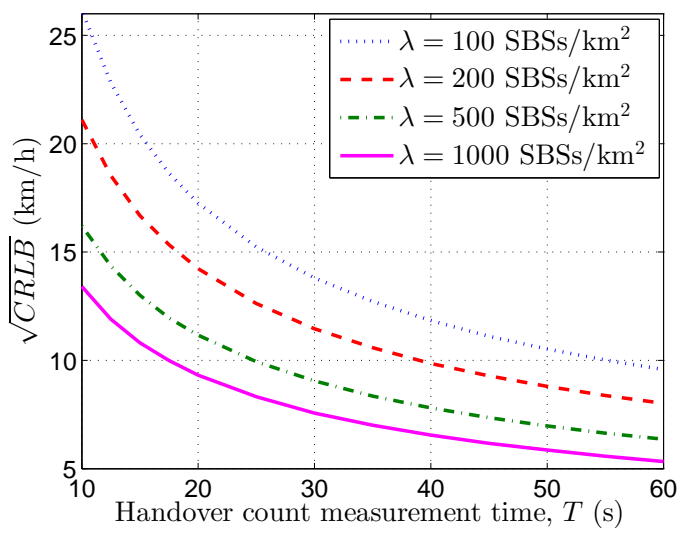

(a) CRLB derived using $f_{H}^{\mathrm{g}}(h)$.

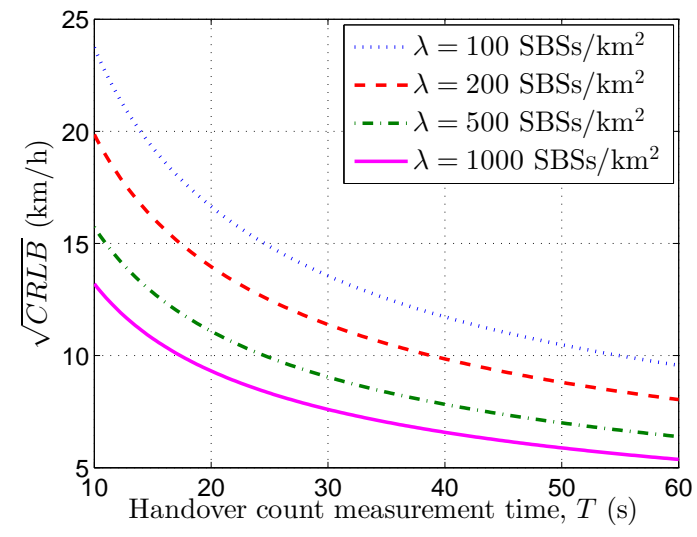

(b) CRLB derived using $f_{H}^{\mathrm{n}}(h)$.

Figure 7.8: CRLB versus $T$ for different $\lambda$ and $v=60 \mathrm{~km} / \mathrm{h}$.

example, with SBS density $\lambda=500 \mathrm{SBSs} / \mathrm{km}^{2}$ and a UE velocity $v=120 \mathrm{~km} / \mathrm{h}$, the CRLB of velocity estimation is just $20 \mathrm{~km} / \mathrm{h}$ as shown in Figure 7.7. After all, it is a choice of the service provider to use a measurement time interval based on the requirements. Therefore, the effects of variations in $T$ are also investigated in the remainder of this chapter.

The effect of handover-count measurement time $T$ on the CRLB can be seen in Fig 7.8. For a given UE velocity and SBS density, CRLB decreases as the measurement time increases. Therefore, longer handover-count measurement time results into better accuracy of velocity estimation, with the assumption that the UE will continue traveling on a linear trajectory.

\subsubsection{Variance of the MVU Velocity Estimator}

The variance of the MVU velocity estimator given in (7.23) is plotted in Figure 7.9. Even though the derived MVU estimator is not an efficient estimator, it can be observed from the plots that the variance of the MVU estimator tightly matches with the CRLB. Therefore, the MVU estimator performs very close to an efficient estimator. 


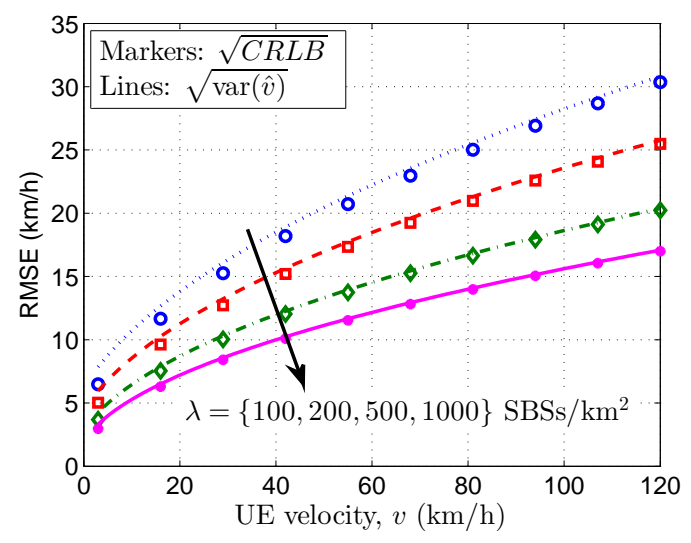

(a) For variations in $v$ and $\lambda$, with $T=12 \mathrm{~s}$

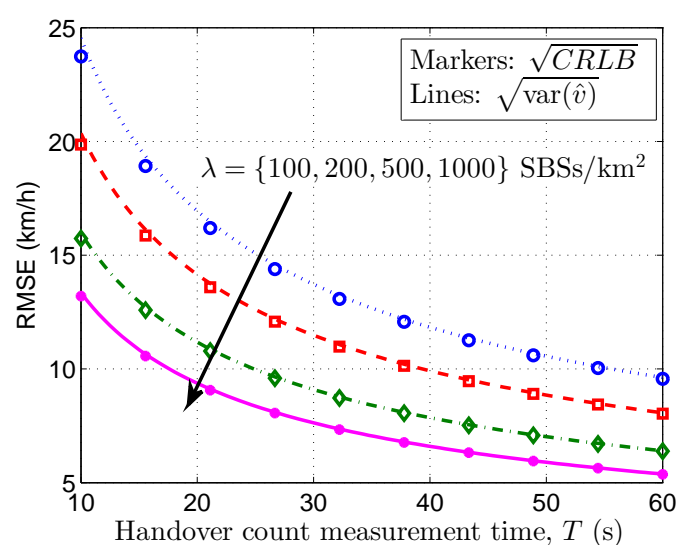

(b) For variations in $T$ and $\lambda$, with $v=60 \mathrm{~km} / \mathrm{h}$

Figure 7.9: Comparison between the CRLB and the variance of the MVU estimator.

\subsubsection{Mobility State Probabilities, and Probabilities of Detection and False Alarm}

In order to classify a UE into one of the three mobility states, the velocity thresholds $v_{1}$ and $v_{\mathrm{u}}$ can be set depending on the requirements of the service provider. We have set $v_{\mathrm{l}}=40 \mathrm{~km} / \mathrm{h}$ and $v_{\mathrm{u}}=80 \mathrm{~km} / \mathrm{h}$. The plots of mobility state probabilities versus UE velocity $v$, for four different $\lambda$ values are shown in Figure 7.10. Here, the analytic plots that were obtained using (7.27)-(7.29) are observed to match accurately with the simulation results. When we examine these plots carefully, it can be noticed that as the SBS density $\lambda$ increases, the slope of the curves during their transitions also increases, which is closer to the ideal case where the slope is equal to infinity. In other words, accuracy of MSD improves significantly with larger SBS densities.

The probabilities of detection and false alarm versus the UE velocity $v$ are shown in Figure 7.11 for different $\lambda$ values. It can be noticed that as the UE velocity nears to any one of the velocity thresholds, the probability of detection decreases and the probability of false alarm increases. The probability of detection is close to 0.5 when the UE velocity is equal to $v_{\mathrm{l}}$ or $v_{\mathrm{h}}$. 


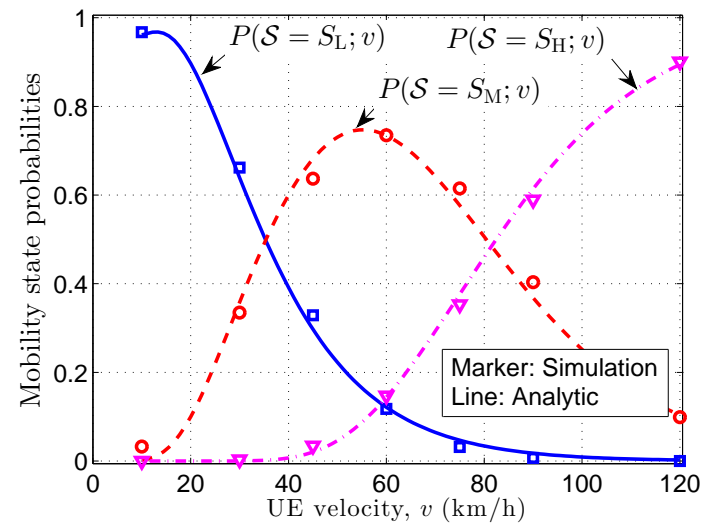

(a) $\lambda=100 \mathrm{SBSs} / \mathrm{km}^{2}$

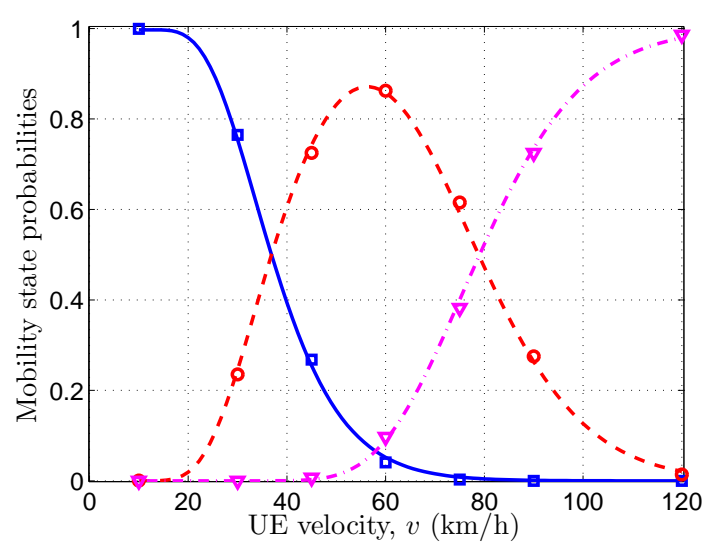

(c) $\lambda=500 \mathrm{SBSs} / \mathrm{km}^{2}$

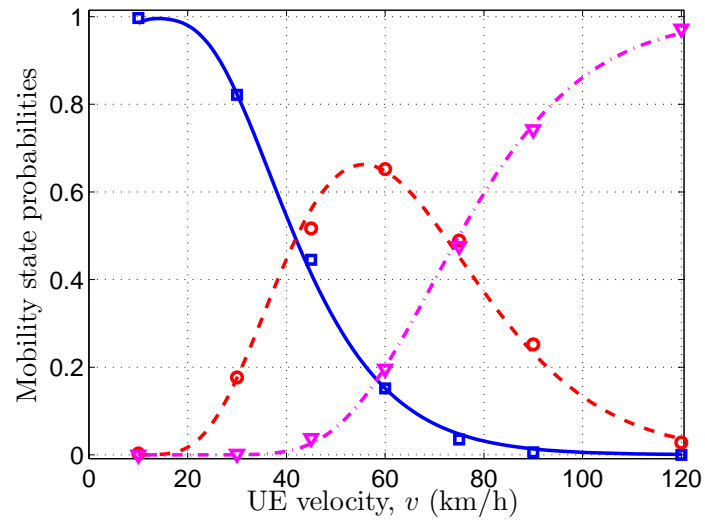

(b) $\lambda=200 \mathrm{SBSs} / \mathrm{km}^{2}$

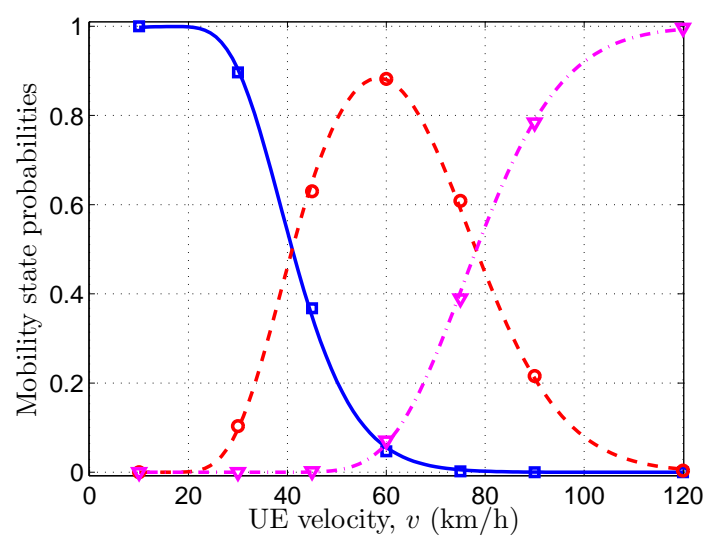

(d) $\lambda=1000 \mathrm{SBSs} / \mathrm{km}^{2}$

Figure 7.10: Mobility state probabilities versus UE velocity; $T=12 \mathrm{~s}, v_{1}=40 \mathrm{~km} / \mathrm{h}$ and $v_{\mathrm{u}}=80 \mathrm{~km} / \mathrm{h}$.

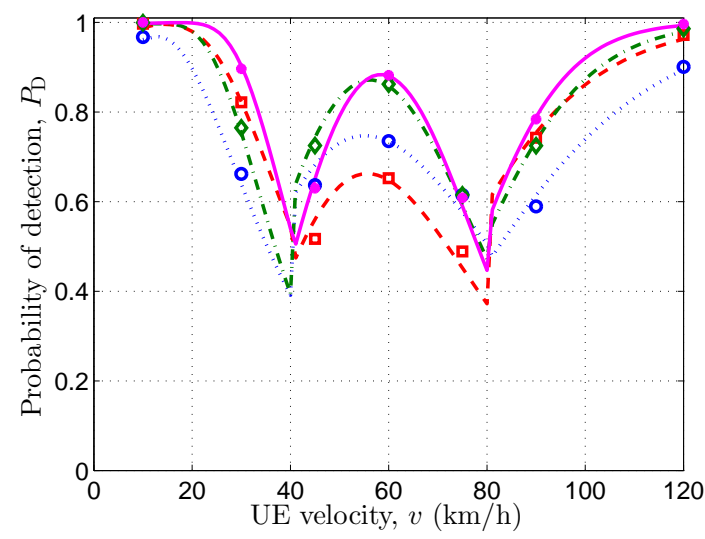

(a) Probability of detection

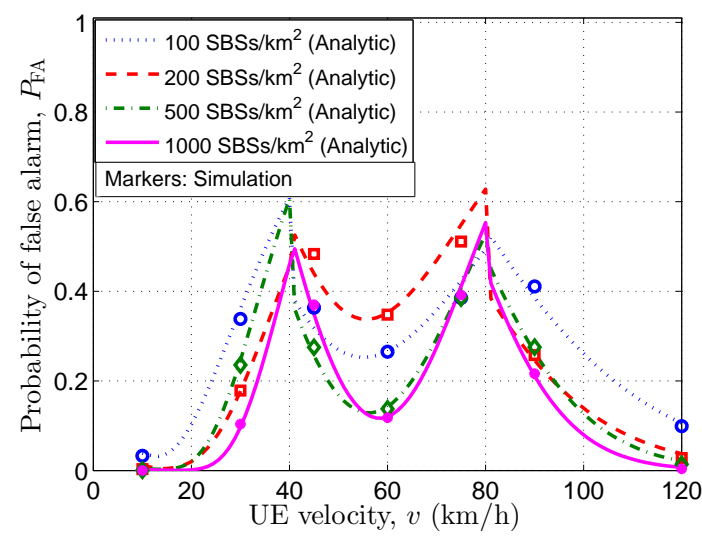

(b) Probability of false alarm

Figure 7.11: Probabilities of detection and false alarm. 
The average probability of detection is plotted in Figure 7.12 for different combinations of the handover count thresholds $h_{1}$ and $h_{\mathrm{u}}$ such that $h_{\mathrm{u}}>h_{\mathrm{l}}$. Here, the probability of detection is averaged over UE velocity in the range of $10 \mathrm{~km} / \mathrm{h}$ to $120 \mathrm{~km} / \mathrm{h}$, with $\lambda=500 \mathrm{SBSs} / \mathrm{km}^{2}$. It can be observed in Figure 7.12 that the average probability of detection maximizes to 0.797 with $h_{\mathrm{l}}=3$ and $h_{\mathrm{u}}=7$. On the other hand, computing (7.30) with $\lambda=500 \mathrm{SBSs} / \mathrm{km}^{2}, T=12 \mathrm{~s}, v_{\mathrm{l}}=40 \mathrm{~km} / \mathrm{h}$ and $v_{\mathrm{u}}=80 \mathrm{~km} / \mathrm{h}$ also results into $h_{\mathrm{l}}=3$ and $h_{\mathrm{u}}=7$. Therefore, equations in (7.30) obtained as a result of our analysis provide analytic expressions for optimum $h_{1}$ and $h_{\mathrm{u}}$ that maximizes the probability of detection.

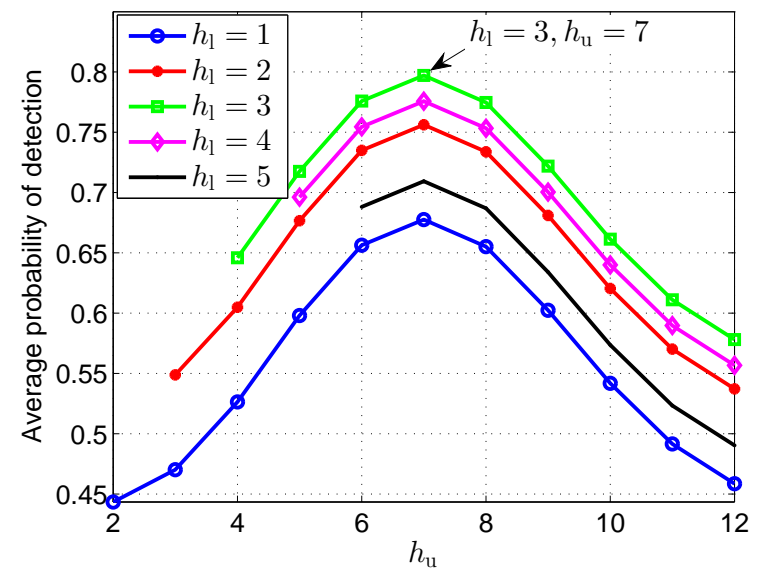

Figure 7.12: Average probability of detection for different combinations of $h_{1}$ and $h_{\mathrm{u}}$, with $\lambda=500 \mathrm{SBSs} / \mathrm{km}^{2}$.

\subsubsection{Estimator Performance with RWP Mobility Model}

In this sub-section, we assess the accuracy of velocity estimator with RWP mobility model for the UE [89]. In the RWP model, the UE movement trace is assumed to have a sequence of quadruples which can be defined as $\left\{\left(\boldsymbol{X}_{n-1}, \boldsymbol{X}_{n}, V_{n}, S_{n}\right)\right\}_{n \in \mathbb{N}}$, where $n$ denotes the $n$ th movement period, $\boldsymbol{X}_{n-1}$ and $\boldsymbol{X}_{n}$ denote the starting and target waypoints, respectively, during the $n$-th movement period, $V_{n}$ denotes the velocity, and $S_{n}$ denotes the pause time at the waypoint $\boldsymbol{X}_{n}$. The angle between two consecutive waypoints is uniformly randomly distributed on $[-\pi, \pi]$, while the transition length $L_{n}=\left\|\boldsymbol{X}_{n}-\boldsymbol{X}_{n-1}\right\|$ between two consecutive waypoints is i.i.d. and Rayleigh distributed with the CDF, $P(L \leq l)=1-\exp \left(-\xi \pi l^{2}\right), l \geq 0$, 
where $\xi$ is defined as the mobility parameter. Larger $\xi$ statistically implies that the transition lengths $L$ are shorter and may be appropriate for mobile users walking. In contrast, smaller $\xi$ statistically implies longer transitions lengths which may be appropriate for driving users.

We performed simulations for a special case of the RWP mobility model in which the UE velocity $V_{n} \equiv v$ is a positive constant, and the pause times $S_{n}=0$. The characteristics of the RMSE of velocity estimator are shown in Figure 7.13. It can be observed that the RMSE increases with increasing $v$, and decreases with increasing $T$, similar to the characteristics of the linear mobility model. The RMSE increases with the increasing mobility parameter $\xi$. For large $\xi$, the UE switches its directions more number of times within the handover count measurement time interval, leading to larger estimation errors. On the other hand, for smaller $\xi$, the UE's direction switch rate is smaller which results into smaller RMSE. As $\xi \rightarrow 0$, the RMSE of RWP mobility model converges to the RMSE of the linear mobility model. This is because the direction switch rate tends to zero, and the UE follows a straight line trajectory indefinitely.
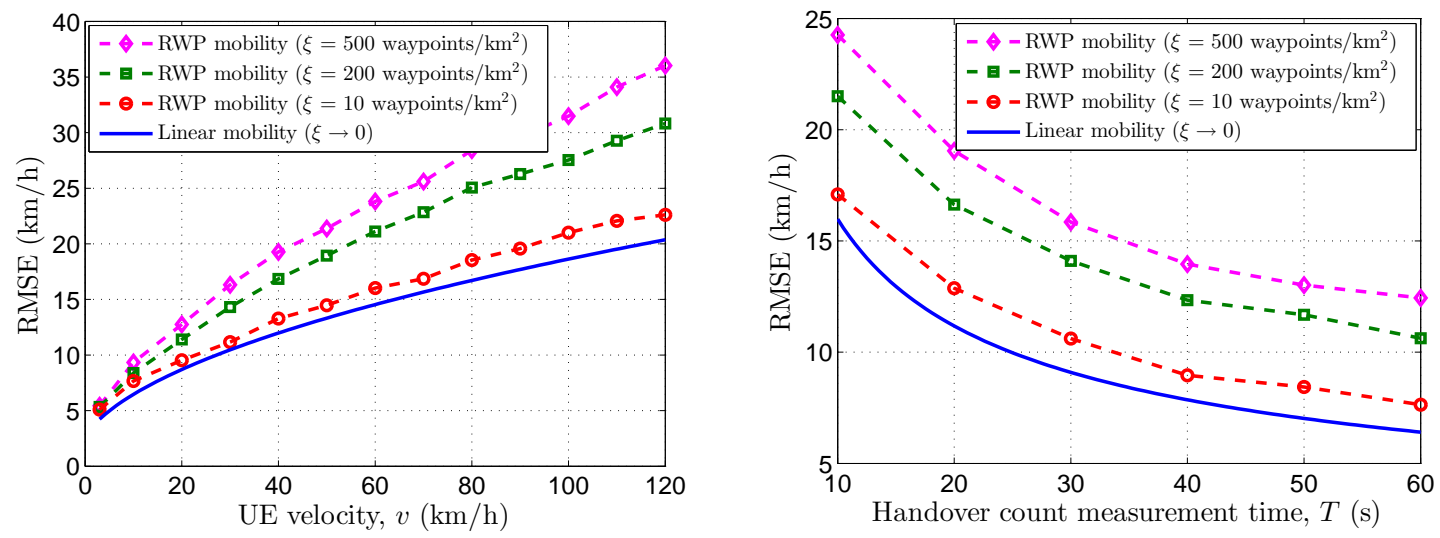

(a) RMSE versus UE velocity, with $T=12 \mathrm{~s}$ and (b) RMSE versus handover count measurement $\lambda=500 \mathrm{SBSs} / \mathrm{km}^{2}$. time, with $v=60 \mathrm{~km} / \mathrm{h}$ and $\lambda=500 \mathrm{SBSs} / \mathrm{km}^{2}$.

Figure 7.13: RMSE performance of the velocity estimator with RWP mobility model. 


\subsubsection{Mobility State Detection with Variable UE Velocity}

In this sub-section, we demonstrate the functionality of our MVU estimator with variable UE velocity, and the effect of handover-count measurement time on estimation accuracy. Consider an example in which a user is traveling in a train that is moving over a straight line trajectory in a downtown region. Assume that the density of small cells is $\lambda=1000 \mathrm{SBSs} / \mathrm{km}^{2}$, and handover counts are measured during regular intervals $T$. The actual velocity of UE in the train, estimated velocity and the detected mobility state are shown in Figure 7.14 during a trip from one station to another station. It can be noticed that the accuracy of velocity estimation is better in Figure $7.14(\mathrm{~b})$ with $T=30 \mathrm{~s}$, when compared to Figure 7.14(a) with $T=12 \mathrm{~s}$. Also, the probability of false alarm is smaller with $T=30 \mathrm{~s}$. However, longer measurement time intervals can lead to lower estimation accuracies if the velocity changes significantly within the measurement time interval.
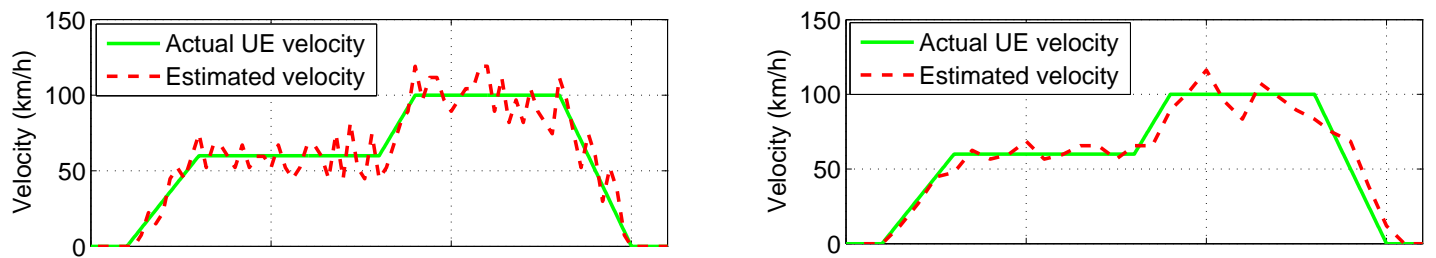

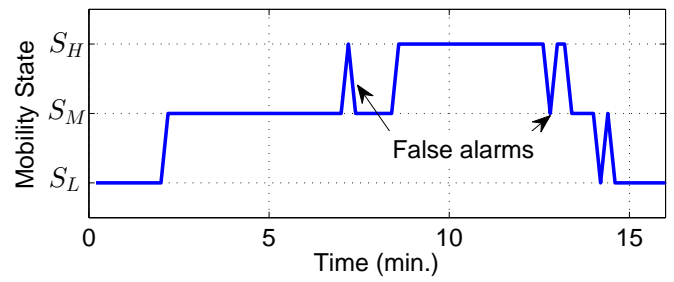

(a) $T=12 \mathrm{~s}$

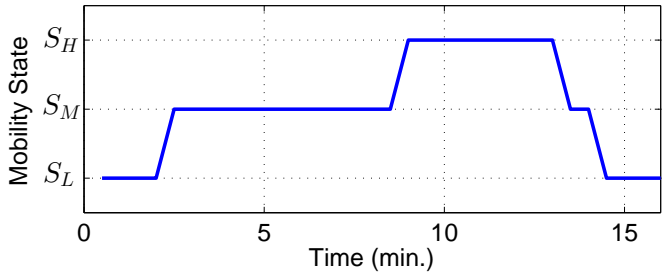

(b) $T=30 \mathrm{~s}$

Figure 7.14: Demonstration of velocity estimation and MSD with variable UE velocity.

\subsubsection{Velocity Estimation in Clustered SBS Deployments}

In practical scenarios, SBSs are deployed more in places where more traffic demand is expected, which results into clustered deployments. In this subsection, we model the clustered deployment of SBSs using Matern-cluster process that can be realized using cluster centers and cluster points. The cluster centers form a Poisson process with intensity $\lambda_{0}$, and the 
cluster points (SBS locations) are located around each cluster center within a disc of radius $R$. The cluster points in these discs are realized using Poisson processes with intensity $\lambda_{1}$.
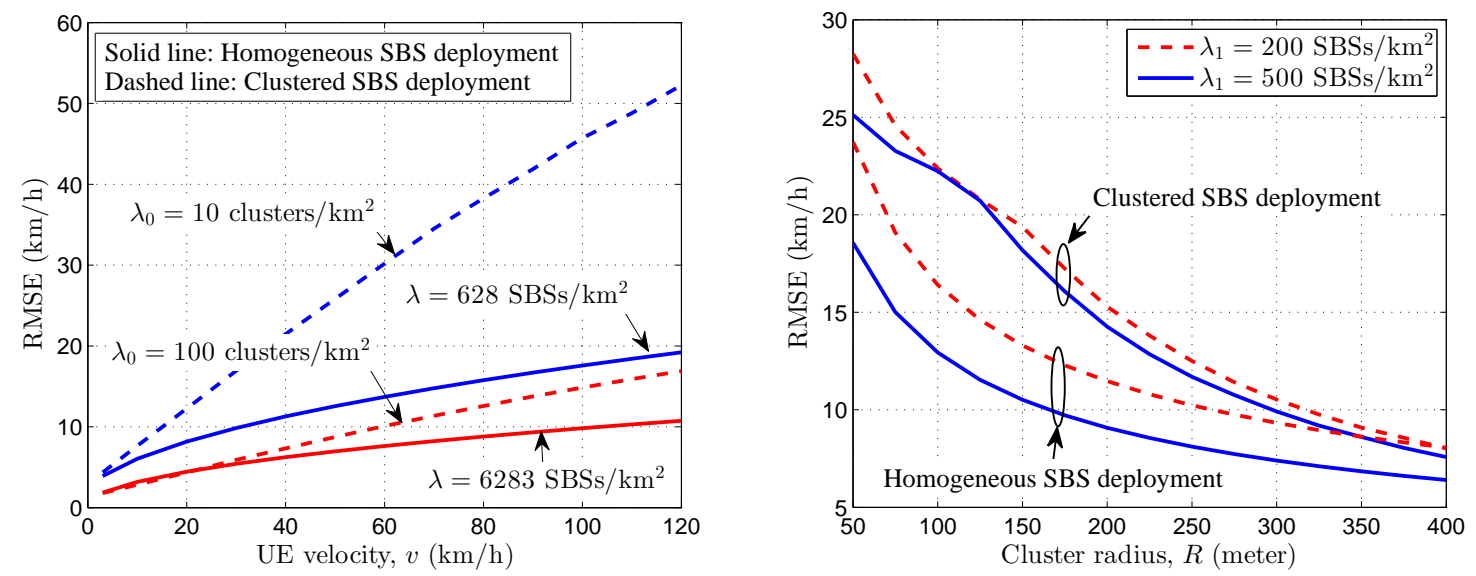

(a) RMSE versus the velocity for different $\lambda_{0}$, with (b) RMSE versus the cluster radius for different $\lambda_{1}=500 \mathrm{SBSs} / \mathrm{km}^{2}, R=200 \mathrm{~m}$, and $T=12 \mathrm{~s} . \quad \lambda_{1}$, with $\lambda_{0}=50$ clusters $/ \mathrm{km}^{2}, v=60 \mathrm{~km} / \mathrm{h}$ and $T=12 \mathrm{~s}$.

Figure 7.15: RMSE of velocity estimation in homogeneous and clustered SBS deployments.

The RMSEs of velocity estimator in homogeneous and clustered deployments of SBSs are compared in Figure 7.15. For fair comparison between the homogeneous and clustered deployments, the intensities of both Poisson and Matern-cluster processes were kept same. Given the parameters $\lambda_{0}, \lambda_{1}$ and $R$ of Matern-cluster process, the equivalent homogeneous intensity $\lambda$ for Poisson process can be found using $\lambda=\lambda_{0} \lambda_{1} \pi R^{2}$. In general, the RMSE is higher in clustered deployment of SBSs due to the heterogeneous nature of Materncluster process and the fact that a UE cannot obtain handover-count measurements outside the discs of clusters. It can be observed in Figure 7.15(a) that the RMSE difference between homogeneous and clustered SBS deployments increases with increasing UE velocity $v$. The RMSE difference also increases significantly when $\lambda_{0}$ decreases, for example, from 100 clusters $/ \mathrm{km}^{2}$ to 10 clusters $/ \mathrm{km}^{2}$. In Figure $7.15(\mathrm{~b})$, it can be seen that the RMSE difference decreases with increasing $R$. 


\subsubsection{Velocity Estimation with Matern Hardcore Process for SBS Locations}

In this sub-section, we model the SBS locations using Matern hardcore process (HCP), in which, the distance between any two points is greater than a hardcore distance $R_{\mathrm{hc}}$. The realization of Matern HCP involves generation of points using a homogeneous PPP, followed by a thinning procedure. For the Matern HCP of type II, the thinning procedure involves associating a random mark to each point of the parent PPP, and a point is deleted if there exists another point within the hardcore distance $R_{\mathrm{hc}}$ with a smaller mark [38]. To realize the HCP with intensity $\lambda_{\text {hc }}$, the intensity of the parent PPP should be

$$
\lambda=\frac{-\ln \left(1-\lambda_{\mathrm{hc}} \pi R_{\mathrm{hc}}^{2}\right)}{\pi R_{\mathrm{hc}}^{2}},
$$

provided that $R_{\mathrm{hc}}$ is smaller than $R_{\mathrm{hc}}^{\max }=1 / \sqrt{\pi \lambda_{\mathrm{hc}}}$. We use $R_{\mathrm{hc}}=\rho / \sqrt{\pi \lambda_{\mathrm{hc}}}$, where $0 \leq \rho<1$ is the randomness parameter. When $\rho=0, R_{\mathrm{hc}}$ is 0 and the HCP is equivalent to PPP in which the points are random located as shown in Figure 7.16(a). When, the $\rho$ increases to 0.99, the points are located more regularly as shown in Figure 7.16(b).
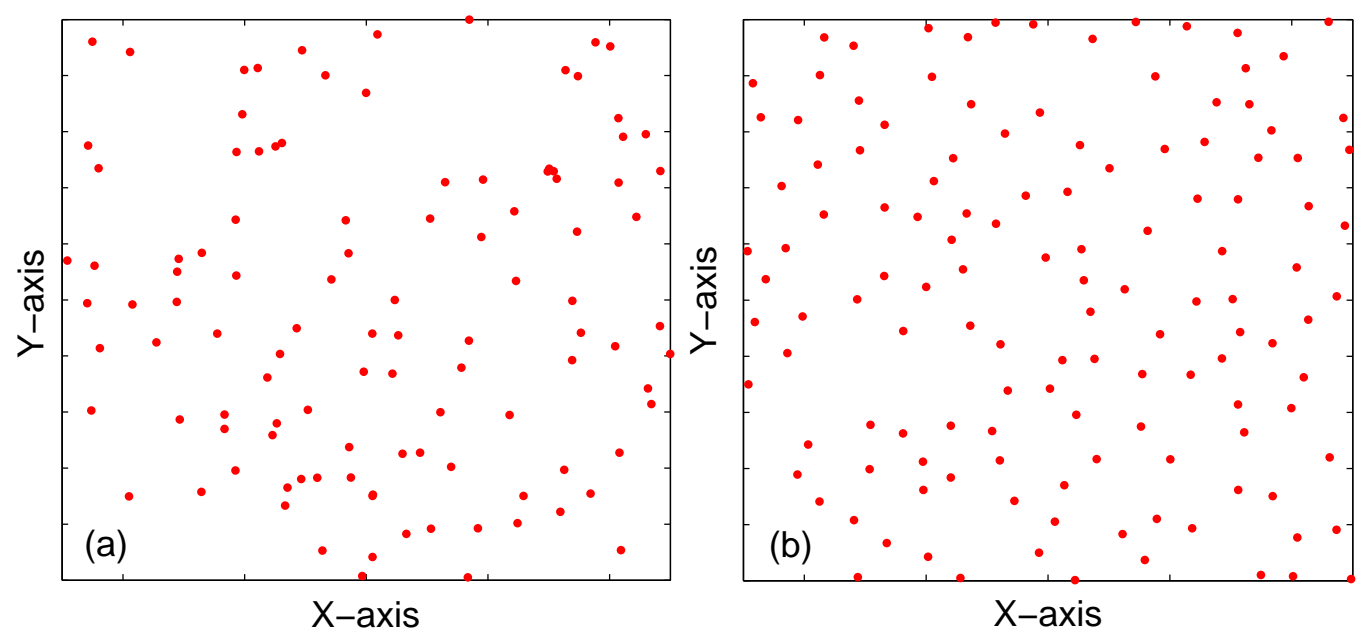

Figure 7.16: Realizations of Matern HCP of type II with fixed $\lambda_{\text {hc }}$ and different $\rho$ values; (a) $\rho=0$; (b) $\rho=0.99$.

The RMSE of the MVU velocity estimator against the variation in $\rho$ is shown in Figure 7.17 for different velocities. The RMSE of the velocity estimator decreases as the $\rho$ 
increases, because the randomness in the SBS locations decreases with increasing $\rho$. Therefore, in scenarios where the SBSs are more uniformly placed, the velocity estimation will work more effectively.

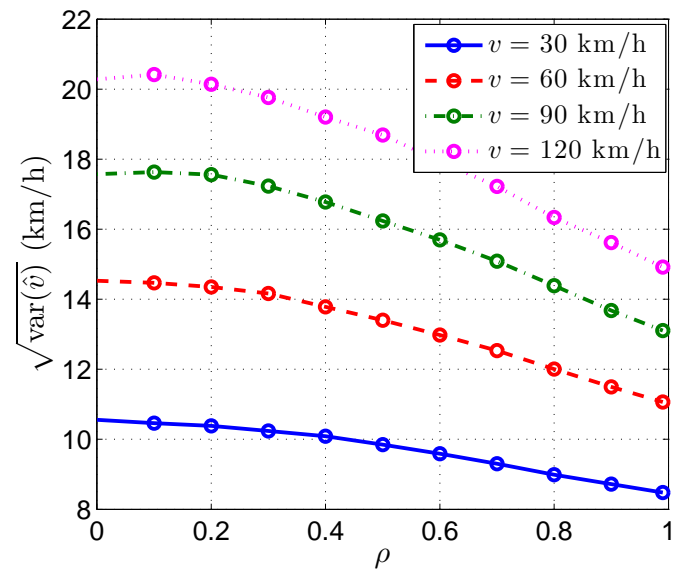

Figure 7.17: RMSE of velocity estimation with type II Matern HCP for the SBS locations. 


\section{CHAPTER VIII Sojourn Time based Velocity Estimation}

The handover-count based velocity estimation of a UE was studies in Chapter VII. Another simple way to estimate a user's velocity is via the use of sojourn time samples. Unlike the handover count velocity estimation method which uses the number of handovers within a given time window, the sojourn time based velocity estimation method can make use

of both the handover-count within a time window and the sojourn times between two consecutive handovers. In this chapter, the CRLB for the sojourn-time based velocity estimation is analyzed. Stochastic geometry is used for the spatial modeling of small cells, and the CRLB is derived using the tools from estimation theory. An asymptotically unbiased velocity estimator is also derived. Our analysis shows that the sojourn time based velocity estimation exhibit a lower CRLB compared to the CRLB of classical velocity estimation using handover count.

The goals of this chapter is to introduce a novel technique for UE velocity estimation based on sojourn-time samples, and analyze its accuracy through CRLBs, when the density of SBSs is known. Our contributions will also include: 1) The derivation of a closed form expression for the JPDF of $N$ sojourn-time samples; 2) the derivation, using the JPDF, of a closed form expression for the CRLB of velocity estimation; and 3) the derivation of an asymptotically unbiased velocity estimator whose accuracy is investigated for various UE speeds and SBS densities.

\subsection{System Model}

Consider a two-tier heterogeneous network (HetNet) consisting of macro cell BSs (MBSs) and SBSs that use different frequency bands, as in the phantom cell architecture $[147,162]$. The SBSs are randomly distributed according to a homogeneous PPP with intensity $\lambda$. Assuming that the SBSs are densely deployed, as expected in future wireless networks [163], the coverage of small cells can be modeled effectively using a Poisson-Voronoi tessellation as 


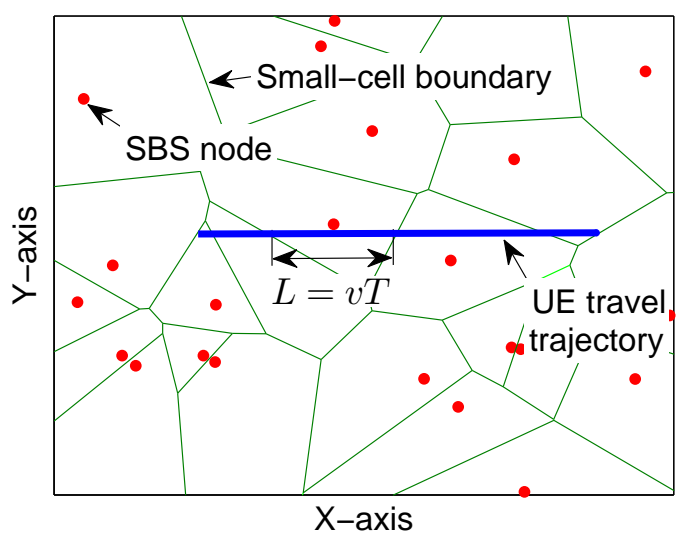

Figure 8.1: Poisson-Voronoi tessellation of small cells and the UE travel trajectory.

shown in Figure 8.1. For simplicity, we assume that the UE travels with a constant velocity $v$ along a linear trajectory as shown in Figure 8.1. We let $T$ be the sojourn time and $L$ the distance traveled within a cell. During the travel, we assume that the UE can detect its boundary crossings, and therefore can measure the sojourn times. Since the SBSs are randomly distributed, the sojourn time $T$ is a continuous random variable.

\subsubsection{Sojourn Time PDF}

The PDF of the distance $L$ traveled by a UE in an arbitrary Poisson-Voronoi cell is derived in $[164]$ as:

$$
\begin{aligned}
& f_{L}(l)=\int_{0}^{\pi} \int_{0}^{\pi-\alpha} \frac{\pi^{2} \lambda l^{2} \rho_{\alpha} \rho_{\beta}\left(2 \pi \lambda l^{2} b_{0}^{2} \rho_{\alpha}^{2}-c_{0}\right)}{\sin (\alpha+\beta)} e^{-\pi \lambda l^{2} V_{2}(\alpha, \beta)} \mathrm{d} \beta \mathrm{d} \alpha \\
& \text { where } V_{2}(\alpha, \beta)=\left(1+\rho_{\beta}^{2}-2 \rho_{\beta} \cos \alpha\right)\left(1-\frac{\beta}{\pi}+\frac{\sin 2 \beta}{2 \pi}\right)+\rho_{\beta}^{2}\left(1-\frac{\alpha}{\pi}+\frac{\sin 2 \alpha}{2 \pi}\right), \\
& \rho_{\alpha}=\frac{\sin \alpha}{\sin (\alpha+\beta)}, \quad \rho_{\beta}=\frac{\sin \beta}{\sin (\alpha+\beta)}, \\
& b_{0}=\frac{(\pi-\beta) \cos \beta+\sin \beta}{\pi}, \text { and } c_{0}=\frac{(\pi-\beta)+\sin \beta \cos \beta}{\pi} .
\end{aligned}
$$

The details of (8.1) can be found in [164]. Therein, $L$ is denoted as the chord length. Next, the PDF of sojourn time will be expressed in terms of the chord length $\operatorname{PDF} f_{L}(\cdot)$. 
Theorem 3. Consider a Poisson-Voronoi tessellation formed by the SBSs distributed according to a PPP of intensity $\lambda$. A UE traveling along a straight line trajectory with constant velocity $v$ has a sojourn time PDF which can be expressed as

$$
f_{T}(t ; v)=v f_{L}(v t)
$$

where the function $f_{L}(\cdot)$ is the chord length PDF given in (8.1).

Proof. The sojourn time is the ratio of distance traveled by the UE in a cell to the velocity of the UE, $T=L / v$. Using this relationship, we can express sojourn time distribution as,

$$
F_{T}(t ; v)=P(T \leq t ; v)=P\left(\frac{L}{v} \leq t\right)=F_{L}(v t)
$$

By differentiating the sojourn time distribution with respect to $t$, we can obtain the sojourn time PDF as,

$$
f_{T}(t ; v)=\frac{\partial F_{T}(t ; v)}{\partial t}=\frac{\partial F_{L}(v t)}{\partial t}=v f_{L}(v t)
$$

The plots of sojourn time PDF for different SBS densities are shown in Figure 8.2 for a UE velocity $v=60 \mathrm{~km} / \mathrm{h}$.

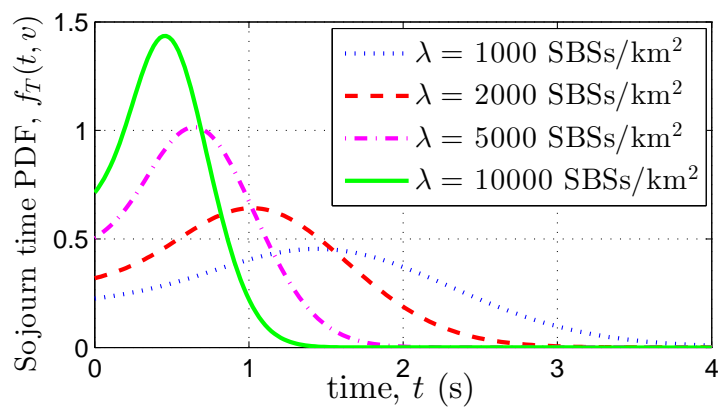

Figure 8.2: Sojourn time PDF for different SBS densities, with $v=60 \mathrm{~km} / \mathrm{h}$.

\subsection{CRLB for Velocity Estimation}

In this section, we derive the CRLB for velocity estimation using the sojourn time samples obtained during a fixed time interval $T_{\mathrm{w}}$. The number of sojourn time samples $N$ is a 
random variable which is equal to the number of handovers during the time interval $T_{\mathrm{w}}$. We first obtain an expression for the JPDF of the $N$ sojourn time samples, and then use this JPDF to derive the CRLB.

Let $\boldsymbol{T}=\left[T_{n}: n=0,1, \ldots, N-1\right]$ be a vector of $N$ sojourn time samples. Using extensive simulations, we have found that the correlation between the sojourn time samples of adjacent cells is less than $5.9 \%$, which is a reasonably small value. Hence, for mathematical tractability, we assume that the sojourn time samples are independent. Therefore, the JPDF of $N$ sojourn time samples will be:

$$
f_{\boldsymbol{T}}(\boldsymbol{t} ; v)=\prod_{n=0}^{N-1} f_{T}\left(t_{n} ; v\right)=v^{N} \prod_{n=0}^{N-1} f_{L}\left(l_{n}\right)
$$

where $\boldsymbol{t}=\left[t_{n} \geq 0: n=0,1, \ldots, N-1\right]$ is a vector parameter, and $l_{n}=v t_{n}$ is the chord length corresponding to the $n$th sojourn time sample. A pre-requisite for the CRLB derivation is that the JPDF must satisfy the regularity condition as will be shown next.

Lemma 3. The JPDF of $N$ sojourn time samples which is shown in (8.3) satisfies the following regularity condition:

$$
E\left[\frac{\partial \log f_{\boldsymbol{T}}(\boldsymbol{t} ; v)}{\partial v}\right]=0 \quad \text { for all } v
$$

with $E[\cdot]$ being the expectation operator.

Proof. See Appendix 6.

Since the JPDF satisfies the regularity condition, the CRLB for velocity estimation can be obtained using

$$
\operatorname{var}(\hat{v}) \geq \frac{1}{E\left[\left(\frac{\partial \log f_{\boldsymbol{T}}(\boldsymbol{t} ; v)}{\partial v}\right)^{2}\right]},
$$

where $\operatorname{var}(\cdot)$ is the variance operator. Next, we show that the CRLB in (8.4) can be expressed in closed form. 
Theorem 4. The CRLB for velocity estimation of a UE using the sojourn time samples measured within a time interval $T_{\mathrm{w}}$ is given by:

$$
\operatorname{var}(\hat{v}) \geq \frac{\pi v}{17.52 T_{\mathrm{w}} \sqrt{\lambda}}
$$

Proof. See Appendix 7.

\subsection{Sojourn Time Based Velocity Estimator}

In this section, we derive an estimator which takes $N$ sojourn time samples as the input and estimates the UE velocity, provided that the SBS density $\lambda$ is known. We start deriving the estimator by using mean sojourn time given by:

$$
E[T]=E\left[\frac{L}{v}\right]=\frac{E[L]}{v}=\frac{\pi}{4 v \sqrt{\lambda}}
$$

The last step in (8.6) was obtained through the expression for mean cross-sectional length which is derived in [153]. By rearranging the terms in (8.6) and using $E[T]=1 / N \sum_{n=0}^{N-1} T_{n}$, we can obtain a velocity estimator as

$$
\hat{v}=\frac{\pi N}{4 \sqrt{\lambda} \sum_{n=0}^{N-1} T_{n}} .
$$

An estimator is said to be unbiased if its expected value is same as the true value of the parameter being estimated. Unbiasedness ensures that, on the average, the estimator yields the true value of the unknown parameter. To check if (8.7) is an unbiased estimator, we derive the expectation as

$$
E[\hat{v}]=\frac{\pi N}{4 \sqrt{\lambda}} E\left[\frac{1}{\sum_{n=0}^{N-1} T_{n}}\right] \neq v
$$




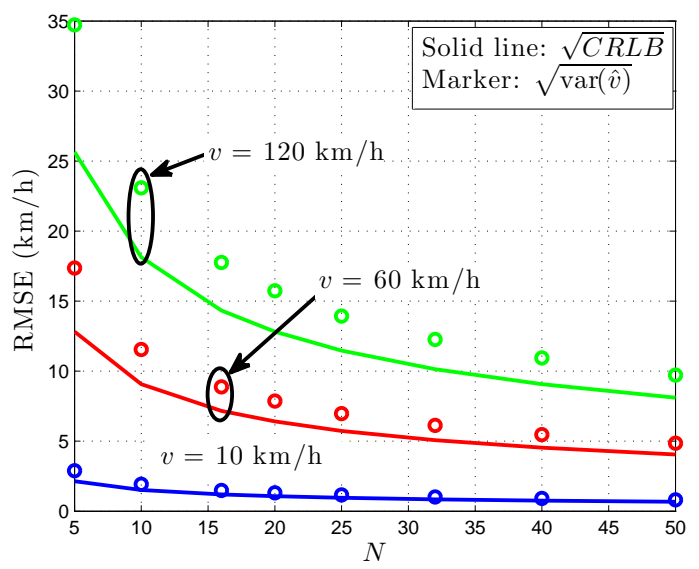

Figure 8.3: CRLB and estimator variance versus $N$, for different UE velocities.

Since $E[\hat{v}] \neq v, \hat{v}$ in (8.7) is not an unbiased estimator. However, as $N$ tends to infinity, we get,

$$
\begin{aligned}
\lim _{N \rightarrow \infty} E[\hat{v}] & =\frac{\pi}{4 \sqrt{\lambda}} E\left[\frac{1}{\lim _{N \rightarrow \infty} \frac{1}{N} \sum_{n=0}^{N-1} T_{n}}\right], \\
& =\frac{\pi}{4 \sqrt{\lambda}} E\left[\frac{1}{E\left[T_{n}\right]}\right]=\frac{\pi}{4 \sqrt{\lambda}} E\left[\frac{4 v \sqrt{\lambda}}{\pi}\right]=v .
\end{aligned}
$$

Therefore, the estimator in (8.7) is asymptotically unbiased.

The variance of the velocity estimator can be expressed as

$$
\begin{aligned}
\operatorname{var}(\hat{v}) & =E\left[\hat{v}^{2}\right]-(E[\hat{v}])^{2} \\
& =\left(\frac{\pi N}{4 \sqrt{\lambda}}\right)^{2}\left(E\left[\left(\frac{1}{\sum_{n=0}^{N-1} T_{n}}\right)^{2}\right]-\left(E\left[\frac{1}{\sum_{n=0}^{N-1} T_{n}}\right]\right)^{2}\right) .
\end{aligned}
$$

\subsection{Numerical Results}

The plots of CRLB and estimator variance are shown in Figure 8.3 for different $N$ and $v$ values, with $\lambda=3000 \mathrm{SBSs} / \mathrm{km}^{2}$. The CRLB plots were obtained using (8.5), while the plots of estimator variance were obtained through numerical computation of (8.10). It can be observed that the root mean squared error (RMSE) increases with the increasing UE 


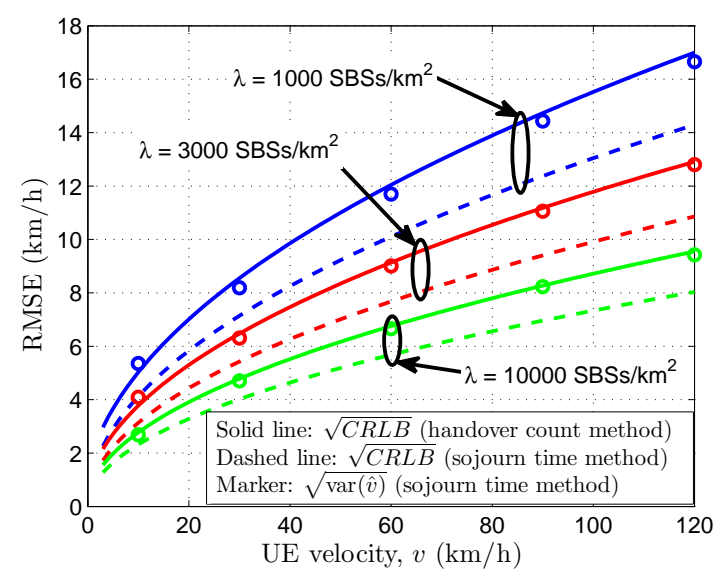

Figure 8.4: Comparison between the RMSEs of handover count and sojourn time methods for the velocity estimation of a UE.

velocity. On the other hand, the RMSE decreases as the number of sojourn time samples increases. Also, as $N$ increases, the difference between the CRLB and the estimator variance is reduced.

Next, we compare the CRLBs of the handover-count based and the sojourn-time based velocity estimation methods. The handover-count based velocity estimation method is analyzed in [165], where a UE's velocity is estimated based on the number of small cells traversed by the UE during a predefined time interval. In [165], CRLB for the handovercount based velocity estimation is derived to be

$$
\operatorname{var}\left(\hat{v}_{\mathrm{ho}}\right) \geq\left[\frac{1}{2}\left(\frac{0.41 T_{\mathrm{w}} \sqrt{\lambda}}{\sigma^{2}}\right)^{2}+\left(\frac{\mu}{v \sigma}\right)^{2}\right]^{-1},
$$

where $\mu=4 v T_{\mathrm{w}} \sqrt{\lambda} / \pi$, and $\sigma^{2}=0.07+0.41 v T_{\mathrm{w}} \sqrt{\lambda}$. The CRLBs of sojourn-time method and handover-count method are compared in Figure 8.4. The measurement time interval is fixed to $T_{\mathrm{w}}=12$ seconds for both the methods. It can be observed in Figure 8.4 that the CRLB of sojourn-time method is smaller than the CRLB of handover-count method, for all the UE velocities and the densities of SBSs. This is because the handover count method estimates the UE velocity by making use of only the handover count information. In contrast, the sojourn time method uses both handover count information and sojourn 
time information for the velocity estimation. Hence, theoretically the sojourn time method provides better accuracy for the UE velocity estimation.

The variance of sojourn-time based velocity estimator is also plotted in Figure 8.4 in comparison with the CRLBs. It can be seen that the estimator variance does not attain the CRLB of sojourn-time method. Deriving an efficient estimator that can attain the CRLB of sojourn-time method is a challenging task due to the complexity of the sojourn-time PDF expression, and it is left as a future work. 


\section{CHAPTER IX \\ Energy Efficiency in HetNets}

In this chapter, we use stochastic geometry to analyze the EE and SE in a two-tier LTEAdvanced HetNet. We consider range expansion and FeICIC which has been standardized in 3GPP Release-11. We optimize system parameters of the HetNet and study the trade-offs associated between EE and SE of the network.

\subsection{System Model}

We consider a two-tier HetNet system with MBS, PBS and UE locations modeled as twodimensional homogeneous PPPs of intensities $\lambda, \lambda^{\prime}$ and $\lambda_{\mathrm{u}}$, respectively. Both the MBSs and the PBSs share a common transmission bandwidth. We assume round robin scheduling in all the downlinks of a cell. We also assume that the cells have full buffer traffic and the thermal noise is negligible when compared to interference.

A REB is added to the RSRP of small cells during UE association, to virtually increase the coverage areas of small cells and improve the offloading of UEs to the picocells. However, the picocell SIR in the range expanded region is lower than the macrocell SIR. As a result, the offloaded UEs in the range expanded region of picocells might experience high interference from the umbrella macrocell. This interference can be mitigated using FeICIC in which the MBS transmits at reduced power during certain subframes, while the PBSs transmit at full power during all the subframes, as illustrated in Fig. 9.1. Here, $\beta$ is the duty cycle of USFs, i.e., ratio of the number of USFs to the total number of sub-frames in a frame. During USFs, the MBS transmits at full power $P_{\text {mbs }}$ and during CSFs, it transmits at a reduced power $\alpha P_{\mathrm{mbs}}$, where $0 \leq \alpha \leq 1$ is the power reduction factor. The PBS transmits with power $P_{\mathrm{pbs}}$ during all the subframes.

For an arbitrary UE, let $r$ and $r^{\prime}$ be the distances from its nearest MBS and PBS, respectively. Then, assuming Rayleigh fading channel, the RSRPs from the nearest MBS and PBS are given by, $S(r)=P_{\mathrm{mbs}} H / r^{\delta}$ and $S^{\prime}\left(r^{\prime}\right)=P_{\mathrm{pbs}} H^{\prime} /\left(r^{\prime}\right)^{\delta}$, respectively. Here, $\delta$ is 


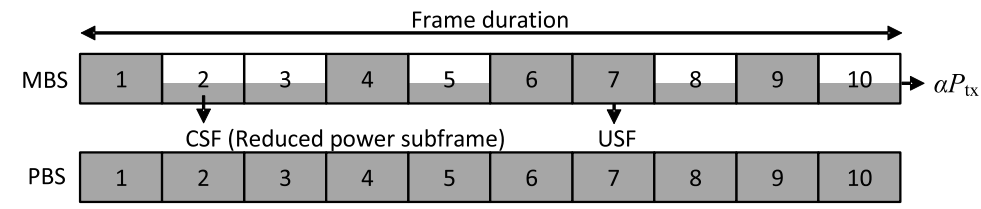

Figure 9.1: Frame structure at MBS and PBS with reduced power subframes.

the path-loss exponent, and the random variables $H \sim \operatorname{Exp}(1)$ and $H^{\prime} \sim \operatorname{Exp}(1)$ account for Rayleigh fading. Define an interference term, $Z$, as the total interference power at a UE from all the MBSs and PBSs, excluding the nearest MBS and PBS. Then, an arbitrary UE experiences the following four SIRs in different subframe types shown in Fig. 9.1:

$$
\begin{aligned}
\Gamma & =\frac{S(r)}{S^{\prime}\left(r^{\prime}\right)+Z}, \rightarrow \text { USF SIR from nearest MBS } \\
\Gamma^{\prime} & =\frac{S^{\prime}\left(r^{\prime}\right)}{S(r)+Z}, \rightarrow \text { USF SIR from nearest PBS } \\
\Gamma_{\mathrm{csf}} & =\frac{\alpha S(r)}{S^{\prime}\left(r^{\prime}\right)+Z}, \rightarrow \text { CSF SIR from nearest MBS } \\
\Gamma_{\mathrm{csf}}^{\prime} & =\frac{S^{\prime}\left(r^{\prime}\right)}{\alpha S(r)+Z} \cdot \rightarrow \mathrm{CSF} \text { SIR from nearest PBS }
\end{aligned}
$$

The cell selection and scheduling process using $\Gamma, \Gamma^{\prime}$ and the REB $\tau$ can be performed using the criteria shown in Fig. 9.2. Here, $\rho$ and $\rho^{\prime}$ are the scheduling thresholds used in macrocell and picocell, respectively. Using the model described in this section, a sample

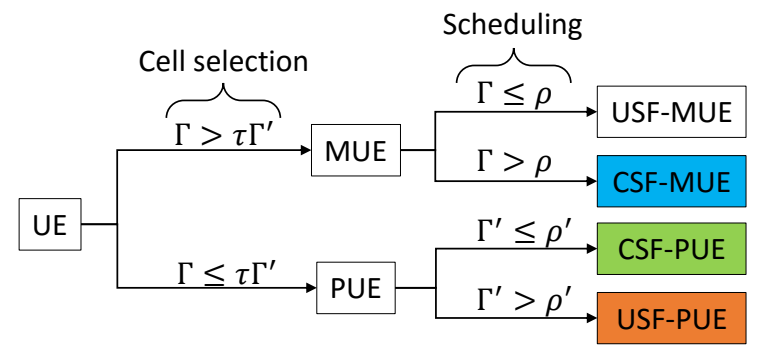

Figure 9.2: Cell selection and scheduling criteria in different subframes.

layout of MBSs and PBSs with their coverage areas for the four different UE categories are illustrated in Fig. 9.3. Here, the coverage regions for USF- and CSF-PUEs in picocells are colored in orange and green, respectively. Whereas in macrocells, the coverage regions for USF- and CSF-MUEs are colored in white and blue, respectively. 


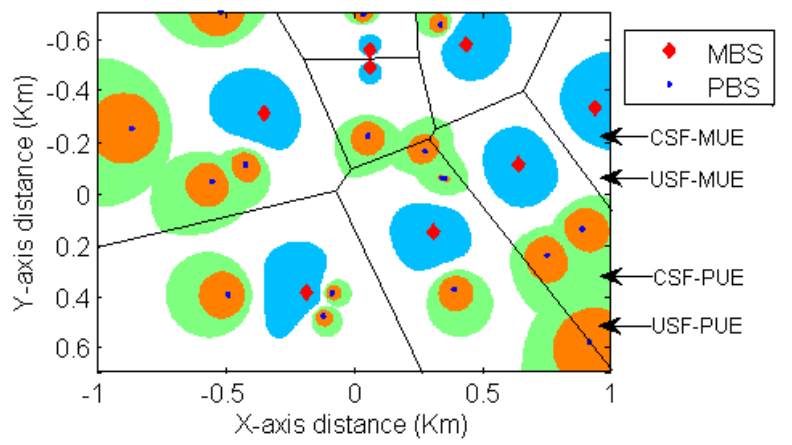

Figure 9.3: Illustration of two-tier HetNet layout.

Using Shannon's capacity formula, the SE of a UE can be expressed as $C=\log _{2}(1+$ $S I R)$, where $S I R$ can be $\Gamma, \Gamma^{\prime}, \Gamma_{\text {csf }}$ or $\Gamma_{\text {usf }}$ depending on the UE's category. The EE in a HetNet can be defined as

$$
E E=\frac{\text { Area SE }}{\text { Area power consumption }}
$$

where, "Area SE" is the achieved SE per unit area in bps $/ \mathrm{Hz} / \mathrm{m}^{2}$, and "Area power consumption" is the power consumption of the network infrastructure per unit area in $\mathrm{W} / \mathrm{m}^{2}$. According to (9.5), increasing the area SE will increase the EE. However, increasing the area SE might involve setting up of more BSs which will also increase the area power consumption. Therefore, there is a trade-off associated between the SE and EE enhancements. We formulate the following two optimization problems,

$$
\max _{\rho, \rho^{\prime}, \beta, \alpha} E E \quad \text { and } \quad \max _{\rho, \rho^{\prime}, \beta, \alpha} C_{\log }=\sum_{i} \log \left(C_{i}\right)
$$

for maximizing the EE and the SE with proportional fair scheduling (PFS), respectively. Here, $C_{i}$ is the SE of user $i$ in a cell, and the summation is over all the users in the cell. The maximization of logarithmic sum capacity, $C_{\log }$, is taken into account, which corresponds to PFS [121]. 


\subsection{Numerical Results}

In this section, we will present the results for joint optimization of the FeICIC parameters for given densities of macrocell/picocell tiers and their respective transmission powers. Preliminary results on joint optimization of parameters $\rho, \rho^{\prime}, \beta$, and $\alpha$ are presented in Fig. 9.4, which shows the maximized EE as a function of PBS density relative to the MBS density. Here, the path loss exponent $\delta=4$ and the transmit powers of MBSs and PBSs are $P_{\mathrm{mbs}}=46 \mathrm{dBm}$ and $P_{\mathrm{pbs}}=30 \mathrm{dBm}$ respectively. Results show that increasing the network density or the REB improves the EE. The EE is about $0.18 \mathrm{bps} / \mathrm{Hz} / \mathrm{W}$ when the PBS density is equal to the MBS density, and the REB does not have noticeable impact on the EE. When the PBS density is increased to ten times the MBS density, the EE increases to $46 \mathrm{bps} / \mathrm{Hz} / \mathrm{W}$ with no REB $(\tau=0 \mathrm{~dB})$ and to $0.55 \mathrm{bps} / \mathrm{Hz} / \mathrm{W}$ with an REB of $12 \mathrm{~dB}$. On the other hand, EE-maximizing selection of $\alpha$ is seen to be decreasing with increasing PBS density, and eventually becomes zero. In other words, for high PBS densities, 3GPP Rel. 10 eICIC with almost blank subframes becomes optimal.
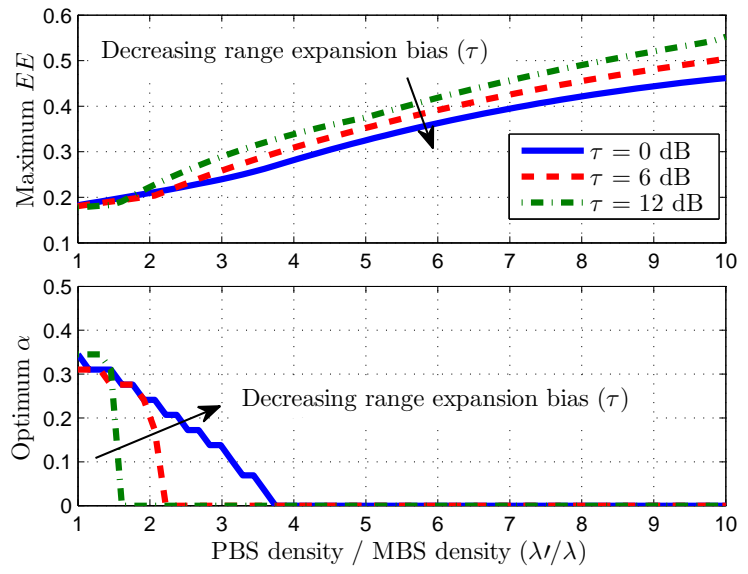

Figure 9.4: Optimum EE and corresponding $\alpha$, versus PBS density.

In Fig. 9.5, the impact of network densification on the SE is investigated in a similar scenario. Fig. 9.5 shows the maximized $C_{\log }$ as a function of PBS density relative to the MBS density. For the same scenario, the optimum $\alpha$ value is also shown as a function of relative PBS density. Two different transmission powers of PBSs (10 dBm and $30 \mathrm{dBm})$ are considered, along with the range expansion bias values of $\{0,6,12\} \mathrm{dB}$. Results show 


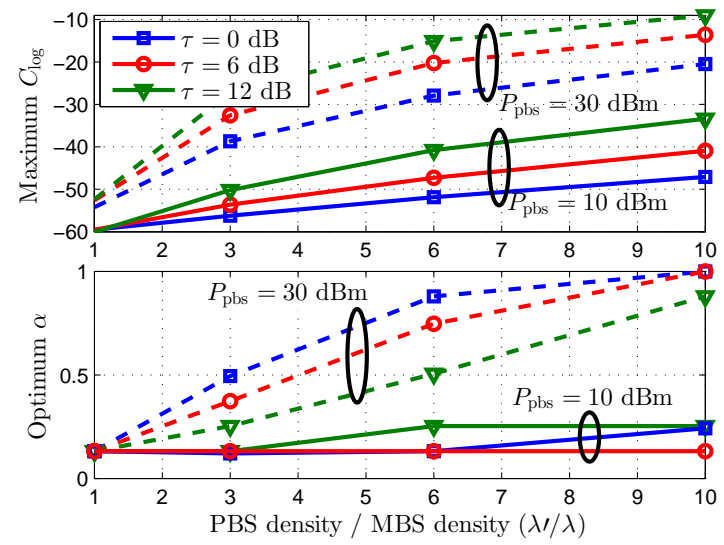

Figure 9.5: Optimum $C_{\log }$ and corresponding $\alpha$, versus PBS density.

that the maximum $C_{\log }$ increases as a function of PBS density, REB, and PBS transmission power. The optimum $\alpha$ increases with increasing PBS density or increasing $P_{\mathrm{pbs}}$. For dense PBS deployments with high transmission powers $(30 \mathrm{dBm})$, results show that not implementing interference coordination becomes preferable, since optimum value of $\alpha$ in Fig. 9.5 converges to 1 (the MBS always transmits will full power in all subframes). On the other hand, for lower transmission powers, interference coordination with $\alpha$ between 0.13 and 0.25 maximizes the logarithmic sum capacity for PBS densities up to $10 \times$ that of PBS density. This behavior of $\alpha$ is in contrast to the EE-optimized $\alpha$ values in Fig. 9.4, and calls for further studies to investigate the design trade-offs for future deployments of dense HetNets, jointly considering the SE and the EE perspectives. 


\section{CHAPTER X \\ Concluding Remarks and Future Work}

The next generation cellular networks are expected to be composed of densely deployed small cells in order to keep up with the exponentially increasing mobile data traffic. There are many challenges associated with such densely deployed HetNets. In this research, we have studied some of the important challenges which can be categorized into capacity enhancement, mobility management, and energy efficiency enhancement. In this chapter, we present the conclusions of this research work and also identify key directions for future research.

In Chapter III, spectral efficiency and 5th percentile throughput expressions were derived for HetNets with reduced power subframes and range expansion. These expressions were validated using the Monte Carlo simulations. Joint optimization of the key system parameters, such as range expansion bias, power reduction factor, scheduling thresholds, and duty cycle of reduced power subframes, was performed to achieve maximum aggregate capacity and proportional fairness among users. Our analysis showed that under optimum parameter settings, the HetNet with reduced power subframes yields better performance than that with almost blank subframes (eICIC) in terms of both aggregate capacity and proportional fairness. However, transmitting the reduced power subframes with greater than half the maximum power proved to be inefficient because it degraded both the aggregate capacity and the proportional fairness. Increasing the range expansion bias improved the proportional fairness but degraded the aggregate capacity. In case of eICIC, the duty cycle of almost blank subframes had a significant effect on the fairness, but with reduced power subframes and optimized scheduling thresholds, duty cycle had a limited effect on fairness. Hence, fixing the duty cycle and optimizing the scheduling thresholds was preferable since it avoids the overhead of coordinating the duty cycle among the cells through the $\mathrm{X} 2$ interface. We also compared the 5 th percentile SE results from PPP model with that of real BS deployment and hexagonal grid model. We observed that the hex grid model 
forms the upper bound while the PPP model forms the lower bound. Increasing the PBS density or the PBS transmit power would improve the 5th percentile SE. In this study, we considered SIR as the only deciding factor for UE association. However in real LTE networks, UE association criteria also include factors such as UE velocity, load conditions in cells, and backhaul capacity. An important direction for future research would be to take such factors into account for capturing a wider range of deployment scenarios.

In Chapter IV, we showed that the public safety communications can significantly benefit from deploying UABSs in the event of any damage to the network infrastructure due to natural calamities or malevolent attacks. Through simulations and genetic algorithm, we showed that SE coverage and 5th percentile SE of the network can be improved significantly by optimally placing the UABSs. Higher path-loss exponent of the channel is shown to provide significant improvement in the SE coverage and 5th percentile SE of the network. The use of REB for UABSs provided higher gains when more number of UAVs were deployed. The potential benefits of using inter-cell interference coordination techniques with the REB are yet to be studied. Other directions for future research include studying the impact of UABSs in generalized HetNet scenario (consisting of small-cells such as picocells and femtocells), studying the delay in transmitted messages with non-full buffer traffic model, and developing path planning algorithms for UAV placement.

In Chapter V, we proposed a novel spectrum market where sharing is promoted explicitly by the government. The government offers subsidy support to the wireless operators and requires a performance metric to be reported. We considered this metric to be the number of outside customers served by the operator, where outside customer means a customer who is not subscribed to that particular operator. This way, the operators are motivated to invest their subsidy support into regions where likelihood of reaching and serving outside customers is higher. This, in turn, increases the aggregate coverage area and the overall signal quality the customers receive. Both of these end results are appealing to the government and are the main reasons why the government should provide subsidy.

Our results showed how the government should influence the spectrum sharing by controlling the subsidy amount allocated to each provider. Using real $B S$ locations from two 
providers in two cities of UK, we studied how the providers can distribute the received subsidy amount into different regions to serve maximum number of outside customers and eventually maximize their revenues. Such a spectrum market will be attractive to the operators and discourage monopolization, while the customers can seamlessly obtain better service from the providers. Furthermore, small providers with relatively smaller infrastructure can survive in this type of market and successfully compete against large operators. In this research work, we presented the results of a simplified spectrum market consisting of two-regions and two-providers. A direction for future research would be to analyze the results of a generalized spectrum market consisting of $R$ regions and $P$ providers. Another research direction would be compare the social welfare of the spectrum subsidy scheme with that of the roaming scheme.

In Chapter VI, we performed channel impulse response measurements in an indoor office environment by using USRPs. A frequency domain channel sounding method was employed to characterize a wide-band channel of bandwidth greater than the USRP supported bandwidth. The channel measurement data was utilized to determine the large-scale and small-scale statistics of the channel, using which, a channel model was developed. Using this channel model in a multi-hop D2D simulator, we analyzed the BER performance of single-hop and two-hop communication scenarios with respect to the distance between the source and destination nodes. Our simulation results showed that the two-hop case provides significant improvement in the BER performance when compared to the single-hop case.

In Chapter VII, we derived a handover-count based MVU estimator for the velocity of a UE, and derived optimum handover-count thresholds for MSD. We provided approximations to the PMF of handover-count using two types of standard distributions: gamma distribution and Gaussian distribution. We found that the PMF approximation using gamma distribution, even though complicated when compared to Gaussian distribution, provided a better fit to the PMF with a smaller MSE. On the other hand, the use of Gaussian distribution provided simpler and closed-form solutions to the PMF approximation. Subsequently, we derived the CRLB of velocity estimation and a MVU velocity estimator whose variance was approximately equal to the CRLB. The CRLB of velocity estimation decreases with the 
increasing time interval for counting the number of handovers, which showed the trade-off between the accuracy and the rapidness of velocity measurements.

The increasing density of small-cells in the future cellular networks facilitates more accurate UE velocity estimation, since the CRLB decreases with the increasing SBS density. Moreover, the probability of MSD also increases with the increasing SBS density. The results with RWP mobility model showed that the accuracy of the velocity estimator decreases with the increasing randomness in UE's mobility. On the other hand, results with Matern-cluster process showed that the estimation accuracy is poorer with clustered SBS deployment when compared to the homogeneous SBS deployment. Future research directions may include: path prediction of UEs, and development of algorithms to dynamically adjust the measurement time interval depending on the past samples of estimated velocity.

In Chapter VIII, we derived a closed-form expression for the CRLB of sojourn-time based velocity estimation, which is a function of small cell density, UE velocity, and measurement time interval. We also derived an asymptotically unbiased velocity estimator. Theoretically, the sojourn-time based velocity estimation was shown to be more accurate than the handover-count based velocity estimation. Future work includes incorporating more realistic mobility model, developing efficient UE velocity estimator that uses sojourn time samples, and modeling uncertainties in the boundary detection of small cells.

In Chapter IX, we analyzed the EE and SE in a two-tier HetNet that uses range expansion and reduced power subframes. The SE and EE in the HetNet can be increased substantially by increasing the density of small cells. The range expansion provides higher gains in the EE and SE of the network when the PBS density is much higher than the MBS density. We showed the trade-off associated with the optimum power level of the reduced power subframes $(\alpha)$ for maximizing EE and maximizing SE with PFS. 


\section{BIBLIOGRAPHY}

[1] Cisco Systems, "Cisco Visual Networking Index: Global Mobile Data Traffic Forecast Update, 2013-2018," White Paper, Feb. 2014.

[2] Qualcomm, "The 1000x mobile data challenge: More small cells, more spectrum, higher efficiency," Presentation Slides, Nov. 2013.

[3] K. Mallinson, "The 2020 vision for LTE," White Paper, Jun. 2012.

[4] D. López-Pérez, I. Guvenc, G. Roche, M. Kountouris, T. Q. Quek, and J. Zhang, "Enhanced inter-cell interference coordination challenges in heterogeneous networks," IEEE Wireless Commun. Mag., vol. 18, no. 3, pp. 22-31, June 2011.

[5] T. Power and L. Strickling, "Supporting innovative approaches to spectrum sharing," Office of Science and Technology Policy website, 2013. [Online]. Available: http://www.whitehouse.gov/blog/2013/03/11/supportinginnovative-approaches-spectrum-sharing

[6] A. M. Mousa, "Challenges of future R\&D in mobile communications," Int. J. Advanced Comput. Sci. and Applicat., vol. 3, no. 10, pp. 1-10, 2012.

[7] J. Andrews, S. Buzzi, W. Choi, S. Hanly, A. Lozano, A. Soong, and J. Zhang, "What will 5G be?" IEEE J. Sel. Areas Commun., vol. 32, no. 6, pp. 1065-1082, June 2014.

[8] 3GPP, "Feasibility study on LTE device to device proximity services - radio aspects," Tech. Rep. (3GPP TR 36.843), 2013.

[9] A. Asadi, Q. Wang, and V. Mancuso, "A survey on device-to-device communication in cellular networks," CoRR, vol. abs/1310.0720, 2013.

[10] Qualcomm, Inc., "Study on LTE device to device proximity services," 3GPP Work Item Description (RP-122009), Spain, Dec. 2012.

[11] D. L. Perez, I. Guvenc, and X. Chu, "Mobility management challenges in 3GPP heterogeneous networks," IEEE Commun. Mag., vol. 50, no. 12, pp. 70-78, Dec. 2012 .

[12] — - "Mobility enhancements for heterogeneous networks through interference coordination," in Proc IEEE Wireless Commun. and Networking Conf. Workshops (WC$N C W)$, Paris, France, April 2012, pp. 69-74.

[13] 3GPP TR 36.839, "Evolved universal terrestrial radio access (E-UTRA); mobility enhancements in heterogeneous networks," Tech. Rep. V11.1.0, Jan. 2013.

[14] Samsung, "Mobility support to pico cells in the co-channel HetNet deployment," Stockholm, Sweden, Mar. 2010, 3GPP Standard Contribution (R2-104017).

[15] J. Puttonen, N. Kolehmainen, T. Henttonen, and J. Kaikkonen, "On idle mode mobility state detection in evloved UTRAN," in Proc. IEEE Int. Conf. Information Technology: New Generations, Las Vegas, NV, Apr. 2009, pp. 1195-1200. 
[16] J. Niu, D. Lee, T. Su, G. Li, and X. Ren, "User classification and scheduling in LTE downlink systems with heterogeneous user mobilities," IEEE Trans. Wireless Commun., vol. 12, no. 12, pp. 6205-6213, December 2013.

[17] J. Niu, T. Su, G. Li, D. Lee, and Y. Fu, "Joint transmission mode selection and scheduling in LTE downlink MIMO systems," IEEE Wireless Commun. Letters, vol. 3, no. 2, pp. 173-176, April 2014.

[18] S. M. Musa and N. F. Mir, "An analytical approach for mobility load balancing in wireless networks," J. Computing and Inform. Technol., vol. 19, no. 3, pp. 169-176, 2011.

[19] A. Dua, F. Lu, and V. Sethuraman, "Speed-adaptive channel quality indicator (cqi) estimation," U.S. Patent 20120163207 A1, June 28, 2012.

[20] A. Prasad, P. Lunden, O. Tirkkonen, and C. Wijting, "Energy-efficient flexible interfrequency scanning mechanism for enhanced small cell discovery," in Proc. IEEE Veh. Technol. Conf. (VTC Spring), Dresden, Germany, June 2013, pp. 1-5.

[21] H. Mutlu, M. Alanyali, and D. Starobinski, "Spot pricing of secondary spectrum access in wireless cellular networks," IEEE/ACM Trans. Netw., vol. 17, no. 6, pp. 1794-1804, Dec 2009.

[22] A. Merwaday, S. Mukherjee, and I. Guvenc, "Capacity analysis of LTE-Advanced HetNets with reduced power subframes and range expansion," EURASIP J. Wireless Commun. Networking, vol. 2014, no. 189, Nov. 2014.

[23] A. Merwaday, I. Guvenc, W. Saad, A. Mehbodniya, and F. Adachi, "Sojourn time based velocity estimation in small cell poisson networks," IEEE Commun. Lett., vol. 20, no. 2, pp. 340-343, Nov. 2015.

[24] A. Merwaday and I. Guvenc, "Handover count based velocity estimation and mobility state detection in dense HetNets," IEEE Trans. Wireless Commun. (TWC), Mar. 2016.

[25] A. Merwaday, A. Tuncer, A. Kumbhar, and I. Guvenc, "Unmanned aerial base stations for public safety communications," submitted to IEEE Veh. Technol. Mag., Jan. 2016.

[26] A. Merwaday, M. Yuksel, T. Quint, I. Guvenc, W. Saad, and N. Kapucu, "Incentivizing spectrum sharing via subsidy regulations," submitted to IEEE Trans. Mobile Computing (TMC), Jan. 2016.

[27] A. Merwaday and I. Guvenc, "Handover count based UE velocity estimation in hyperdense heterogeneous wireless networks," in Proc. IEEE Workshop on Heterogeneous and Small Cell Networks (HetsNets), Co-located with IEEE Global Telecommun. Conf. (GLOBECOM), San Diego, CA, Dec. 2015, pp. 1-6.

[28] — - "UAV assisted heterogeneous networks for public safety communications," in Proc. IEEE Workshop on D2D and Public Safety Commun. (WDPC), Co-located with IEEE Wireless Commun. and Networking Conf. (WCNC), New Orleans, LA, Mar. 2015, pp. 329-334. 
[29] A. Merwaday, N. Rupasinghe, I. Guvenc, W. Saad, and M. Yuksel, "USRP-based indoor channel sounding for D2D and multi-hop communications," in Proc. IEEE Wireless and Microwave Technol. Conf. (WAMICON), Tampa, FL, June 2014, pp. $1-6$.

[30] A. Merwaday, S. Mukherjee, and I. Guvenc, "Hetnet capacity with reduced power subframes," in Proc. IEEE Wireless Commun. and Networking Conf. (WCNC), Istanbul, Turkey, Apr. 2014, pp. 1380-1385.

[31] — - "On the capacity analysis of hetnets with range expansion and eICIC," in Proc. IEEE Global Telecommun. Conf. (GLOBECOM), Atlanta, GA, Dec. 2013, pp. 42574262.

[32] M. Simsek, A. Merwaday, N. Correal, and I. Guvenc, "Device-to-device discovery based on 3GPP system level simulations," in Proc. Int. Workshop on D2D Commun. With and Without Infrastructure, Co-located with IEEE Global Telecommun. Conf. (GLOBECOM), Atlanta, GA, Dec. 2013, pp. 555-560.

[33] A. Merwaday and I. Guvenc, "Optimization of energy efficiency and spectrum efficiency in HetNets," IET Electronics Lett., Apr. 2016.

[34] H. Elsawy, E. Hossain, and M. Haenggi, "Stochastic geometry for modeling, analysis, and design of multi-tier and cognitive cellular wireless networks: A survey," Communications Surveys Tutorials, IEEE, vol. 15, no. 3, pp. 996-1019, Third 2013.

[35] J. Andrews, F. Baccelli, and R. Ganti, "A tractable approach to coverage and rate in cellular networks," IEEE Transactions on Communications, vol. 59, no. 11, pp. 3122-3134, Nov. 2011.

[36] R. Ganti, F. Baccelli, and J. Andrews, "A new way of computing rate in cellular networks," in Proceedings of the IEEE Int. Conf. Commun. (ICC), Kyoto, Japan, June 2011, pp. 1-5.

[37] J. Andrews, R. Ganti, M. Haenggi, N. Jindal, and S. Weber, "A primer on spatial modeling and analysis in wireless networks," Communications Magazine, IEEE, vol. 48, no. 11, pp. 156-163, November 2010.

[38] M. Haenggi, "Mean interference in hard-core wireless networks," IEEE Commun. Lett., vol. 15, no. 8, pp. 792-794, 2011.

[39] M. Haenggi, J. Andrews, F. Baccelli, O. Dousse, and M. Franceschetti, "Stochastic geometry and random graphs for the analysis and design of wireless networks," $S e$ lected Areas in Communications, IEEE Journal on, vol. 27, no. 7, pp. 1029-1046, September 2009.

[40] S. Mukherjee, "Distribution of downlink SINR in heterogeneous cellular networks," IEEE J. Select. Areas Commun. (JSAC), Special Issue on Femtocell Networks, vol. 30, no. 3, pp. 575-585, Apr. 2012.

[41] R. Heath, M. Kountouris, and T. Bai, "Modeling heterogeneous network interference using poisson point processes," IEEE Trans. Signal Process., vol. 61, no. 16, pp. 4114-4126, Aug. 2013. 
[42] T. Novlan, R. Ganti, and J. Andrews, "Coverage in two-tier cellular networks with fractional frequency reuse," in Proceedings of the IEEE Global Telecommun. Conf. (GLOBECOM), Houston, TX, Dec. 2011, pp. 1-5.

[43] H. S. Dhillon, R. K. Ganti, F. Baccelli, and J. G. Andrews, "Modeling and analysis of K-tier downlink heterogeneous cellular networks," IEEE J. Select. Areas Commun. (JSAC), Special Issue on Femtocell Networks, vol. 30, no. 3, pp. 550-560, Apr. 2012.

[44] S. Mukherjee, "Downlink SINR distribution in a heterogeneous cellular wireless network with biased cell association," in Proceedings of the IEEE Int. Conf. Commun. (ICC), Ottawa, Canada, June 2012, pp. 6780 -6786.

[45] H.-S. Jo, Y. J. Sang, P. Xia, and J. Andrews, "Outage probability for heterogeneous cellular networks with biased cell association," in Proceedings of the IEEE Global Telecommun. Conf. (GLOBECOM), Houston, TX, Dec. 2011, pp. 1-5.

[46] C.-H. Lee, C.-Y. Shih, and Y.-S. Chen, "Stochastic geometry based models for modeling cellular networks in urban areas," Springer Wireless Networks, vol. 19, no. 6, pp. 1063-1072, 2013. [Online]. Available: http://dx.doi.org/10.1007/s11276012-0518-0

[47] OpenCellID website. [Online]. Available: www.opencellid.org

[48] S. Singh and J. Andrews, "Joint resource partitioning and offloading in heterogeneous cellular networks," IEEE Trans. Wireless Commun., vol. 13, no. 2, pp. 888-901, February 2014.

[49] S. Singh, H. S. Dhillon, and J. G. Andrews, "Offloading in heterogeneous networks: Modeling, analysis, and design insights," IEEE Trans. Wireless Commun., vol. 12, no. 5, pp. 2484-2497, May 2013.

[50] S. Mukherjee and I. Guvenc, "Effects of range expansion and interference coordination on capacity and fairness in heterogeneous networks," in Proceedings of the IEEE Asilomar Conf. Sig., Syst., Computers, vol. 1, Monterey, CA, Nov. 2011, pp. 18551859.

[51] Panasonic, "Performance study on ABS with reduced macro power," 3GPP TSGRAN WG1, Tech. Rep. R1-113806, Nov. 2011.

[52] A. Morimoto, N. Miki, and Y. Okumura, "Investigation of inter-cell interference coordination applying transmission power reduction in heterogeneous networks for LTEadvanced downlink," IEICE Trans. on Commun., vol. E96-B, no. 6, pp. 1327-1337, June 2013.

[53] M. Al-Rawi, J. Huschke, and M. Sedra, "Dynamic protected-subframe density configuration in LTE heterogeneous networks," in Proceedings of the IEEE Int. Conf. Computer Commun. Net. (ICCCN), Munich, Germany, 2012, pp. 1-6.

[54] "Overview of 3gpp release 11 v0.1.7," Dec. 2013. [Online]. Available: http://www.3gpp.org/ftp/Information/WORK_PLAN/Description_Releases/Rel11_description_20131224.zip 
[55] G. Baldini, S. Karanasios, D. Allen, and F. Vergari, "Survey of wireless communication technologies for public safety," IEEE Commun. Surveys Tutorials, vol. 16, no. 2, pp. 619-641, Second quarter 2014.

[56] United States Government Accountability Office, "Emergency communications: Various challenges likely to slow implementation of a public safety broadband network," Report to Congressional Requesters, Feb. 2012. [Online]. Available: http://www.gao.gov/assets/590/588795.pdf

[57] A. Kumbhar, F. Koohifar, I. Güvenç, and B. Mueller, "A survey on legacy and emerging technologies for public safety communications," CoRR, vol. abs/1509.08316, 2015. [Online]. Available: http://arxiv.org/abs/1509.08316

[58] About FirstNet, Website, National Telecommunications and Information Administration, US Dept. of Commerce. [Online]. Available: http://www.ntia.doc.gov/page/about-firstnet

[59] N. B. Johnson, "Are drones and robots the future of public safety?" June 2014. [Online]. Available: http://www.statetechmagazine.com/article/2014/06/are-dronesand-robots-future-public-safety

[60] J. Villasenor, "Drones and the future of domestic aviation," Proc. of the IEEE, vol. 102, no. 3, pp. 235-238, Mar. 2014.

[61] C. Barrado, R. Messeguer, J. López, E. Pastor, E. Santamaria, and P. Royo, "Wildfire monitoring using a mixed air-ground mobile network," IEEE Perv. Computing, vol. 9, no. 4, pp. 24-32, 2010.

[62] "Amazon Prime Air." [Online]. Available: http://www.amazon.com/b?node= 8037720011

[63] L. P. Koh, “A drone's-eye view of conservation," June 2013. [Online]. Available: http://www.ted.com/talks/lian_pin_koh_a_drone_s_eye_view_of_conservation

[64] "Google Loon project." [Online]. Available: http://www.google.com/loon/

[65] A. Abdulsalam, H. Li, and J. Zhang, "Internet for all: Stratospheric solutions by Google Loon and Facebook drone," Technical Report, Mar. 2014.

[66] "Aerial base stations with opportunistic links for unexpected and temporary events." [Online]. Available: http://www.absolute-project.eu/

[67] "Spectrum sharing," NTIA, June 2014. [Online]. Available: http://www.ntia.doc.gov /category/spectrum-sharing

[68] "Spectrum-sharing plan approved by FCC," National Journal, Aug. 2012. [Online]. Available: http://www.nationaljournal.com/blogs/techdailydose/2012/08/spectrumsharing-plan-approved-by-fcc-15

[69] M. M. Sohul, M. Yao, T. Yang, and J. H. Reed, "Spectrum access system for the citizen broadband radio service," IEEE Commun. Mag., vol. 53, no. 7, pp. 18-25, 2015. 
[70] H. Celebi, I. Guvenc, S. Gezici, and H. Arslan, "Cognitive-radio systems for spectrum, location, and environmental awareness," IEEE Antennas and Propagation Mag., vol. 52, no. 4, pp. 41-61, Aug. 2010.

[71] R. Zhang, Y.-C. Liang, and S. Cui, "Dynamic resource allocation in cognitive radio networks," IEEE Signal Processing Mag., vol. 27, no. 3, pp. 102-114, 2010.

[72] B. Wang and K. Liu, "Advances in cognitive radio networks: A survey," IEEE J. Select. Topics in Signal Processing, vol. 5, no. 1, pp. 5-23, 2011.

[73] S. M. Yu and S.-L. Kim, "Guaranteeing user welfare in network service: Comparison of two subsidy schemes," Performance Evaluation Review, vol. 40, no. 2, pp. 22-25, September 2012.

[74] N. Jesuale, "Lights and sirens broadband - how spectrum pooling, cognitive radio, and dynamic prioritization modeling can empower emergency communications, restore sanity and save billions," in Proc. IEEE Symp. New Frontiers in Dynamic Spectrum Access Networks (DySPAN), May 2011, pp. 467-475.

[75] U. Kelkar and N. Aerrabotu, "Over-the-air subsidy lock resolution," USPTO Patent US 7088988 B2, 2006.

[76] A. Toor, "Europe puts an end to mobile roaming charges," The Verge, Oct. 2015. [Online]. Available: http://www.theverge.com/2015/10/27/9620346/europeanparliament-mobile-roaming-charges-law

[77] S. Fabrizi and B. Wertlen, "Roaming in the mobile internet," Telecommunications Policy, vol. 32, no. 1, pp. 50-61, 2008. [Online]. Available: http://www.sciencedirect.com/science/article/pii/S0308596107001024

[78] L. Giupponi, R. Agusti, J. Perez-Romero, and O. Sallent, "Improved revenue and radio resource usage through inter-operator joint radio resource management," in Proc. IEEE Int. Conf. Commun. (ICC), 2007, pp. 5793-5800.

[79] H.-B. Chang and K.-C. Chen, "Cooperative spectrum sharing economy for heterogeneous wireless networks," in Proc. IEEE Global Telecommunications Conf. (GLOBECOM) Workshops, 2011, pp. 458-463.

[80] R. Berry, M. Honig, T. Nguyen, V. Subramanian, H. Zhou, and R. Vohra, "On the nature of revenue-sharing contracts to incentivize spectrum-sharing," in Proc. IEEE INFOCOM, 2013, pp. 845-853.

[81] S. Barbera, P. Michaelsen, M. Saily, and K. Pedersen, "Improved mobility performance in LTE co-channel hetnets through speed differentiated enhancements," in Proc. IEEE Globecom Workshops (GC Wkshps), 2012, pp. 426-430.

[82] 3GPP, "Evolved universal terrestrial radio access (E-UTRA); radio resource control (RRC); protocol specification, (TS 36.331)."

[83] 3GPP TS 36.304, "User Equipment (UE) procedures in idle mode," 3GPP-TSG RAN, bb, Tech. Rep. 8.5.0, Mar. 2009. 
[84] M. Ishii and M. Iwamura, "User device and method in mobile communication system," European Patent No: 2187671 A1, May 2010. [Online]. Available: https://www.google.com/patents/EP2187671A1

[85] A. Sampath and J. Holtzman, "Estimation of maximum Doppler frequency for handoff decisions," in Proc. IEEE Veh. Technol. Conf. (VTC), Secaucus, NJ, May 1993, pp. $859-862$.

[86] B. Zhou and S. Blostein, "Estimation of maximum Doppler frequency for handoff decisions," in Proc. Sixth Can. Workshop on Info. Theory, June 1999, pp. 111-114.

[87] L. Zhou and S. D. Blostein, "Recursive maximum likelihood estimation of maximum Doppler frequency of a sampled fading signal," in Proc. Biennial Sym. on Commun., 2000, pp. 361-365.

[88] J. Pang, Q. JIANG, G. Shen, and D. Wang, "Method, apparatus, and system for biasing adjustment of cell range expansion," Oct. 2 2014, wO Patent App. PCT/IB2014/000,572. [Online]. Available: http://www.google.com/patents/WO2014155197A1?cl=en

[89] X. Lin, R. Ganti, P. Fleming, and J. Andrews, "Towards understanding the fundamentals of mobility in cellular networks," IEEE Trans. Wireless Commun., vol. 12, no. 4, pp. 1686-1698, April 2013.

[90] W. Bao and B. Liang, "Handoff rate analysis in heterogeneous cellular networks: A stochastic geometric approach," in Proc. ACM Int. Conf. on Modeling, Analysis and Simulation of Wireless and Mobile Systems, Montreal, QC, Canada, Sept. 2014, pp. 95-102.

[91] Y. Hong, X. Xu, M. Tao, J. Li, and T. Svensson, "Cross-tier handover analyses in small cell networks: A stochastic geometry approach," in Proc. IEEE Int. Conf. Commun. (ICC), London, UK, June 2015, pp. 3429-3434.

[92] S. Sadr and R. Adve, "Handoff rate and coverage analysis in multi-tier heterogeneous networks," IEEE Trans. Wireless Commun., vol. PP, no. 99, pp. 1-1, Jan. 2015.

[93] A. Nadembega, A. Hafid, and T. Taleb, "A destination and mobility path prediction scheme for mobile networks," IEEE Trans. Veh. Technol., vol. 64, no. 6, pp. 25772590, 2015.

[94] _ - "Mobility-prediction-aware bandwidth reservation scheme for mobile networks," IEEE Trans. Veh. Technol., vol. 64, no. 6, pp. 2561-2576, 2015.

[95] T. Anagnostopoulos, C. Anagnostopoulos, and S. Hadjiefthymiades, "Efficient location prediction in mobile cellular networks," Int. J. Wireless Inform. Networks, vol. 19, no. 2, pp. 97-111, 2012.

[96] J. Pan, S. Pan, J. Yin, L. Ni, and Q. Yang, "Tracking mobile users in wireless networks via semi-supervised colocalization," IEEE Trans. Pattern Analysis and Machine Intelligence, vol. 34, no. 3, pp. 587-600, 2012. 
[97] X. Lin, R. Ganti, P. Fleming, and J. Andrews, "Towards understanding the fundamentals of mobility in cellular networks," IEEE Trans. Wireless Commun., vol. 12, no. 4, pp. 1686-1698, Apr. 2013.

[98] Y.-U. Chung, D.-J. Lee, D.-H. Cho, and B.-C. Shin, "Macrocell/microcell selection schemes based on a new velocity estimation in multitier cellular system," IEEE Trans. Vehic. Technol., vol. 51, no. 5, pp. 893-903, Sep. 2002.

[99] M. Gudmundson, "Cell planning in Manhattan environments," in Proc. IEEE Vehic. Technol. Conf. (VTC), Denver, CO, May 1992, pp. 435-438.

[100] "Earth - energy aware radio and network technologies." [Online]. Available: https://www.ict-earth.eu/

[101] Z. Hasan, H. Boostanimehr, and V. Bhargava, "Green cellular networks: A survey, some research issues and challenges," IEEE Commun. Surveys Tutorials, vol. 13, no. 4, pp. 524-540, Nov. 2011.

[102] F. Richter, A. Fehske, and G. Fettweis, "Energy efficiency aspects of base station deployment strategies for cellular networks," in IEEE Vehicular Technol. Conf. (VTC), Anchorage, AK, Sept 2009, pp. 1-5.

[103] F. Richter and G. Fettweis, "Cellular mobile network densification utilizing micro base stations," in IEEE International Conf. Commun. (ICC), Cape Town, South Africa, May 2010, pp. 1-6.

[104] S. Tombaz, M. Usman, and J. Zander, "Energy efficiency improvements through heterogeneous networks in diverse traffic distribution scenarios," in International ICST Conf. Commun. and Networking in China (CHINACOM), Harbin, China, Aug 2011, pp. $708-713$.

[105] L. Saker, S.-E. Elayoubi, L. Rong, and T. Chahed, "Capacity and energy efficiency of picocell deployment in LTE-A networks," in IEEE Vehicular Technol. Conf. (VTC), Budapest, Hungary, May 2011, pp. 1-5.

[106] A. Saeed, A. Akbari, M. Dianati, and M. A. Imran, "Energy efficiency analysis for LTE macro-femto HetNets," in Proc. European Wireless Conf. (EW), Guildford, UK, April 2013, pp. 1-5.

[107] P. Luoto, P. Pirinen, and M. Latva-aho, "Energy efficient load sharing in LTE-A HetNets," in IEEE Int. Conf. Wireless and Mobile Computing, Networking and Commun. (WiMob), Lyon, France, Oct 2013, pp. 119-123.

[108] S. Samarakoon, M. Bennis, W. Saad, and M. Latva-aho, "Opportunistic sleep mode strategies in wireless small cell networks," in Proc. IEEE International Conference on Communications (ICC), Sydney, NSW, June 2014, pp. 2707-2712.

[109] Y. Fu, Z. Fei, N. Wang, C. Xing, and L. Wan, "An energy-efficient dense picostation deployment and power control strategy for heterogeneous networks," Smart Computing Review, vol. 3, no. 1, pp. 24-32, Feb. 2013. 
[110] Y. S. Soh, T. Quek, M. Kountouris, and H. Shin, "Energy efficient heterogeneous cellular networks," IEEE J. Select. Areas Commun. (JSAC), vol. 31, no. 5, pp. 840$850,2013$.

[111] D. Cao, S. Zhou, and Z. Niu, "Optimal combination of base station densities for energy-efficient two-tier heterogeneous cellular networks," IEEE Trans. Wireless Commun., vol. 12, no. 9, pp. 4350-4362, 2013.

[112] W. Nie, F. Zheng, X. Wang, S. Jin, and W. Zhang, "Energy efficiency of cross-tier base station cooperation in heterogeneous cellular networks," Submitted to the IEEE Trans. Wireless Commun. (TWC), 2014.

[113] H. Wang, J. Jiang, J. Li, M. Ahmed, and M. Peng, "High energy efficient heterogeneous networks: Cooperative and cognitive techniques," Hindawi Int. J. Antennas and Propagation, vol. 2013, p. 7, 2013.

[114] S. Mukherjee and H. Ishii, "Energy efficiency in the phantom cell enhanced local area architecture," in Proc. IEEE Wireless Commun. Networking Conf., 2013, pp. $1267-1272$.

[115] D. Tsilimantos, J.-M. Gorce, and E. Altman, "Stochastic analysis of energy savings with sleep mode in OFDMA wireless networks," in Proc. IEEE INFOCOM, Turin, Italy, April 2013, pp. 1097-1105.

[116] X. Zhang, Z. Su, Z. Yan, and W. Wang, "Energy-efficiency study for two-tier heterogeneous networks (HetNet) under coverage performance constraints," Mobile Networks and Applications, vol. 18, no. 4, pp. 567-577, 2013.

[117] Mpact lab data management. [Online]. Available: http://www.mpact.fiu.edu/datamanagement/

[118] S. Mukherjee, "UE coverage in LTE macro network with mixed CSG and open access femto overlay," in Proceedings of the IEEE Int. Conf. Commun. (ICC) Workshops, Kyoto, Japan, June 2011, pp. 1-6.

[119] S. M. Yu and S.-L. Kim, "Downlink capacity and base station density in cellular networks," in Proceedings of the IEEE SpaSWiN workshop (in conjunction with WiOpt), Tsukuba Science City, Japan, May 2013, pp. 119-124.

[120] J. Moller and D. Stoyan, "Stochastic geometry and random tessellations," Research Report R-2007-28, Department of Mathematical Sciences, Aalborg University, 2007.

[121] P. Viswanath, D. Tse, and R. Laroia, "Opportunistic beamforming using dumb antennas," IEEE Trans. Inf. Theory, vol. 48, no. 6, pp. 1277-1294, Aug. 2002.

[122] M. R. Jeong and N. Miki, "A comparative study on scheduling restriction schemes for lte-advanced networks," in Proceedings of the IEEE 23rd Int. Symp. Personal Indoor and Mobile Radio Communications (PIMRC), Sydney, Australia, Sept. 2012, pp. 488-495. 
[123] M. Simsek, M. Bennis, and I. Guvenc, "Enhanced intercell interference coordination in HetNets: Single vs. multiflow approach," in Proc. IEEE Globecom Workshops (GC Wkshps), Atlanta, GA, Dec 2013, pp. 725-729.

[124] M. Simsek, M. Bennis, and A. Czylwik, "Dynamic inter-cell interference coordination in hetnets: A reinforcement learning approach," in IEEE Global Communications Conference (GLOBECOM), Anaheim, CA, Dec 2012, pp. 5446-5450.

[125] "Backhauling x2," Cambridge Broadband Networks, Apr. 2011. [Online]. Available: http://cbnl.com/sites/all/files/userfiles/files/Backhauling-X2.pdf

[126] Sitefinder website. [Online]. Available: http://www.sitefinder.ofcom.org.uk

[127] M. Kobayashi, "Experience of infrastructure damage caused by the great east Japan earthquake and countermeasures against future disasters," IEEE Commun. Mag., vol. 52, no. 3, pp. 23-29, Mar. 2014.

[128] C. Essid, "Nationwide public safety broadband network," Dept. of Homeland Security, Office of Emergency Communications Report, June 2012. [Online]. Available: http://www.dhs.gov/sites /default/files/publications/Fact\%20Sheet_Nationwide\%20Public \%20Safety\%20Broadband\%20Network.pdf

[129] J. H. Holland, Adaptation in Natural and Artificial Systems. Ann Arbor: University of Michigan Press, 1975.

[130] S. Rohde, M. Putzke, and C. Wietfeld, "Ad hoc self-healing of OFDMA networks using UAV-based relays," Ad Hoc Networks, vol. 11, no. 7, pp. 1893-1906, 2013.

[131] E. Yanmaz, S. Hayat, J. Scherer, and C. Bettstetter, "Experimental performance analysis of two-hop aerial 802.11 networks," in Proc. IEEE Wireless Commun. and Net. Conf (WCNC), Istanbul, Turkey, 2014.

[132] M. Shubik, The Theory of Money and Financial Institutions. Cambridge, MA: MIT Press, 2011, vol. 3.

[133] T. Quint and M. Shubik, Barley, Gold, and Fiat: A Pure Theory of Money (in press). Yale University Press, 2013.

[134] Z. Han, D. Niyato, W. Saad, T. Basar, and A. Hjorungnes, Game Theory in Wireless and Communication Networks: Theory, Models, and Applications. Cambridge University Press, 2011.

[135] S. Chowdhury, D. Kovenock, and R. Sheremeta, "An experimental investigation of colonel blotto games," Economic Theory, vol. 52, pp. 833-861, 2013.

[136] D. Kovenock and B. Roberson, ser. Oxford Handbook of the Economics of Peace and Conflict. New York: Oxford University Press, 2010, ch. Conflicts with Multiple Battlefields, pp. 503-531. 
[137] E. Dechenau, D. Kovenock, and R. Sheremeta, "A survey of experimental research on contests, all-pay auctions, and tournaments," Chapman University, Economic Science Institute, working paper number 12-22, 2012.

[138] M. J. Osborne, An Introduction to Game Theory. Oxford University Press, 2004.

[139] S. Lang, Analysis I. Addison-wesley, 1968.

[140] National Instruments. (2014, Feb. 14) NI USRP. [Online]. Available: https://www.ni.com/usrp/

[141] R. W. Heath, "Introduction to wireless digital communication: A signal processing perspective," .

[142] C. Gentile, N. Golmie, K. Remley, C. Holloway, and W. Young, "A channel propagation model for the $700 \mathrm{MHz}$ band," in Proc. IEEE Int. Conf. Commun. (ICC), Cape Town, South Africa, May 2010, pp. 1-6.

[143] L. Greenstein, S. Ghassemzadeh, S.-C. Hong, and V. Tarokh, "Comparison study of UWB indoor channel models," IEEE Trans. Wireless Commun., vol. 6, no. 1, pp. 128-135, Jan. 2007.

[144] S. Ghassemzadeh, L. Greenstein, T. Sveinsson, A. Kavcic, and V. Tarokh, "UWB delay profile models for residential and commercial indoor environments," IEEE Trans. Veh. Technol., vol. 54, no. 4, pp. 1235-1244, July 2005.

[145] N. Udar, K. Kant, R. Viswanathan, and D. Cheung, "Ultra wideband channel characterization and ranging in data centers," in Proc. IEEE Int. Conf. Ultra-Wideband (ICUWB), Singapore, Sep. 2007, pp. 322-327.

[146] A. Alvarez, G. Valera, M. Lobeira, R. Torres, and J. Garcia, "New channel impulse response model for UWB indoor system simulations," in Proc. IEEE Veh. Technol. Conf. (VTC), vol. 1, Apr. 2003, pp. 1-5 vol.1.

[147] H. Ishii, Y. Kishiyama, and H. Takahashi, "A novel architecture for LTE-B :Cplane/U-plane split and phantom cell concept," in Proc. IEEE Global Telecommun. Conf. (GLOBECOM) Workshops, Anaheim, CA, Dec. 2012, pp. 624-630.

[148] Nokia Networks, "Evolution to ultra-dense networks." [Online]. Available: http://networks.nokia.com/portfolio/latest-launches/evolution-to-ultra-densenetworks

[149] H.-S. Park, A.-S. Park, J.-Y. Lee, and B.-C. Kim, "Two-step handover for LTE hetnet mobility enhancements," in Proc. IEEE Int. Conf. ICT Convergence (ICTC), Jeju Island, Korea, Oct 2013, pp. 763-766.

[150] D. Lopez-Perez, I. Guvenc, and X. Chu, "Theoretical analysis of handover failure and ping-pong rates for heterogeneous networks," in Proc. IEEE Int. Conf. Commun. (ICC), Ottawa, ON, June 2012, pp. 6774-6779. 
[151] NTT DOCOMO, "5G radio access: Requirements, concept and technologies," White Paper, July 2014. [Online]. Available: https://www.nttdocomo.co.jp/english/binary /pdf/corporate/technology/whitepaper_5g/DOCOMO_5G_White_Paper.pdf

[152] Huawei, HiSilicon, "Efficient discovery of small cells and the configurations," Tech. Rep. 3GPP TSG RAN R1-130895, Apr. 2013.

[153] J. Moller, Lectures On Random Voronoi Tessellations. Springer-Verlag, 1994.

[154] M. Tanemura, "Statistical distributions of poisson voronoi cells in two and three dimensions," Forma, vol. 18, no. 4, pp. 221-247, 2003.

[155] J.-S. Ferenc and Z. Nda, "On the size distribution of poisson voronoi cells," Physica A: Statistical Mechanics and its Applications, vol. 385, no. 2, pp. 518 - 526, 2007.

[156] S. M. Kay, Fundamentals of Statistical Signal Processing: Estimation Theory. Prentice-Hall, Inc., 1993.

[157] 3GPP, "Overview of 3GPP release 8," Tech. Rep. V0.3.3, Sep. 2014.

[158] Huawei, HiSilicon, "Further evaluation on enhancements of mobility state estimation in hetnet," Tech. Rep. 3GPP TSG RAN R2-121250, Mar. 2012.

[159] M. Mehta, N. Akhtar, and A. Karandikar, "Enhanced mobility state estimation in LTE hetnets," in Proc. National Conf. Commun. (NCC), Mumbai, India, Feb 2015, pp. 1-6.

[160] H. Shen, K. Liu, D. Xiao, and Y. He, "The enhancements of UE mobility state estimation in the Het-Net of the LTE-A system," in Proc. Int. Conf. Information Technol. and Software Engineering, ser. Lecture Notes in Electrical Engineering, W. Lu, G. Cai, W. Liu, and W. Xing, Eds. Springer Berlin Heidelberg, 2013, vol. 210, pp. 99-106.

[161] J. Turkka, T. Henttonen, and T. Ristaniemi, "Self-optimization of LTE mobility state estimation thresholds," in Proc. IEEE Wireless Commun. and Networking Conf. Workshops (WCNCW), Istanbul, Turkey, April 2014, pp. 161-165.

[162] A. Mohamed, O. Onireti, M. Imran, A. Imran, and R. Tafazolli, "Control-data separation architecture for cellular radio access networks: A survey and outlook," IEEE Commun. Surveys Tutorials, vol. PP, no. 99, pp. 1-1, Jun. 2015.

[163] National Institute of Standards and Technology (NIST), "Ultra-dense networks." [Online]. Available: http://www.nist.gov/ctl/wireless-networks/ultra-dense-nets.cfm

[164] L. Muche, "Contact and chord length distribution functions of the poisson-voronoi tessellaton in high dimensions," Advances in Applied Probability, vol. 42, no. 1, pp. 48-68, Mar. 2010.

[165] A. Merwaday and I. Guvenc, "Handover count based velocity estimation and mobility state detection in dense HetNets," CoRR, vol. abs/1506.08248, Jun. 2015. 
APPENDICES 


\section{Appendix 1: Derivation of JCCDF Expression}

This part of the appendix derives closed form equation for the JCCDF in (3.12). Let us start by rewriting the JCCDF expression,

$$
\mathbb{P}\left\{\Gamma>\gamma, \Gamma^{\prime}>\gamma^{\prime} \mid R=r, R^{\prime}=r^{\prime}\right\}=\mathbb{E}_{Z}\left[\int_{y 1}^{+\infty} f_{\mathrm{Y}}(y) \int_{\gamma(y+Z)}^{y / \gamma^{\prime}-Z} f_{\mathrm{X}}(x) \mathrm{d} x \mathrm{~d} y\right]
$$

where,

$$
\begin{aligned}
f_{\mathrm{X}}(x) & =\lambda_{\mathrm{x}} \exp \left(-\lambda_{\mathrm{x}} x\right) & \text { and } & f_{\mathrm{Y}}(y) & =\lambda_{\mathrm{y}} \exp \left(-\lambda_{\mathrm{y}} y\right) ; \\
\lambda_{\mathrm{x}} & =\frac{r^{\delta}}{P} & \text { and } & \lambda_{\mathrm{y}} & =\frac{\left(r^{\prime}\right)^{\delta}}{P^{\prime}} .
\end{aligned}
$$

The inner integral in (A1.1) can be derived as,

$$
\int_{\gamma(y+Z)}^{y / \gamma^{\prime}-Z} f_{\mathrm{X}}(x) \mathrm{d} x=\exp \left[-\lambda_{\mathrm{x}} \gamma(y+Z)\right]-\exp \left[-\lambda_{\mathrm{x}}\left(\frac{y}{\gamma^{\prime}}-Z\right)\right]
$$

Then, the outer integral in (A1.1) can be derived as,

$$
\begin{aligned}
\int_{y 1}^{+\infty} f_{\mathrm{Y}}(y) \int_{\gamma(y+Z)}^{y / \gamma^{\prime}-Z} f_{\mathrm{X}}(x) \mathrm{d} x \mathrm{~d} y= & \lambda_{\mathrm{y}} \int_{y 1}^{+\infty} \exp \left[-\lambda_{\mathrm{y}} y-\lambda_{\mathrm{x}} \gamma(y+Z)\right] \mathrm{d} y \\
& -\lambda_{\mathrm{y}} \int_{y 1}^{+\infty} \exp \left[-\lambda_{\mathrm{y}} y-\lambda_{\mathrm{x}}\left(\frac{y}{\gamma^{\prime}}-Z\right)\right] \mathrm{d} y
\end{aligned}
$$

The first term in right hand side (RHS) of (A1.5) can be evaluated as,

$$
\lambda_{\mathrm{y}} \int_{y 1}^{+\infty} \exp \left[-\lambda_{\mathrm{y}} y-\lambda_{\mathrm{x}} \gamma(y+Z)\right] \mathrm{d} y=\frac{1}{1+\frac{\gamma \lambda_{\mathrm{x}}}{\lambda_{\mathrm{y}}}} \exp \left[\frac{-\lambda_{\mathrm{x}} \gamma Z\left(1+\gamma^{\prime}\right)-\lambda_{\mathrm{y}} \gamma^{\prime} Z(1+\gamma)}{1-\gamma \gamma^{\prime}}\right]
$$

The second term in RHS of (A1.5) can be evaluated as,

$\lambda_{\mathrm{y}} \int_{y 1}^{+\infty} \exp \left[-\lambda_{\mathrm{y}} y-\lambda_{\mathrm{x}}\left(\frac{y}{\gamma^{\prime}}-Z\right)\right] \mathrm{d} y=\frac{1}{1+\frac{\lambda_{\mathrm{x}}}{\gamma^{\prime} \lambda_{\mathrm{y}}}} \exp \left[\frac{-\lambda_{\mathrm{x}} \gamma Z\left(1+\gamma^{\prime}\right)-\lambda_{\mathrm{y}} \gamma^{\prime} Z(1+\gamma)}{1-\gamma \gamma^{\prime}}\right]$ 
By substituting (A1.6) and (A1.7) in the first and second terms of (A1.5) respectively, we get

$$
\begin{aligned}
\int_{y 1}^{+\infty} f_{\mathrm{Y}}(y) & \int_{\gamma(y+Z)}^{y / \gamma^{\prime}-Z} f_{\mathrm{X}}(x) \mathrm{d} x \mathrm{~d} y \\
& =\left(\frac{1}{1+\frac{\gamma \lambda_{\mathrm{x}}}{\lambda_{\mathrm{y}}}}-\frac{1}{1+\frac{\lambda_{\mathrm{x}}}{\gamma^{\prime} \lambda_{\mathrm{y}}}}\right) \exp \left[\frac{-\lambda_{\mathrm{x}} \gamma Z\left(1+\gamma^{\prime}\right)-\lambda_{\mathrm{y}} \gamma^{\prime} Z(1+\gamma)}{1-\gamma \gamma^{\prime}}\right] .
\end{aligned}
$$

Substituting (A1.8) in (A1.1) and using (A1.3) we get,

$$
\begin{aligned}
& \mathbb{P}\left\{\Gamma>\gamma, \Gamma^{\prime}>\gamma^{\prime} \mid R=r, R^{\prime}=r^{\prime}\right\} \\
& =\left(\frac{1}{1+\gamma \frac{P^{\prime}}{P}\left(\frac{r}{r^{\prime}}\right)^{\delta}}-\frac{1}{1+\gamma^{\prime} \frac{P}{P^{\prime}}\left(\frac{r^{\prime}}{r}\right)^{\delta}}\right) \mathbb{E}_{Z}\left[\exp \left(-Z \frac{\frac{\gamma\left(1+\gamma^{\prime}\right) r^{\delta}}{P}+\frac{\gamma^{\prime}(1+\gamma)\left(r^{\prime}\right)^{\delta}}{P^{\prime}}}{1-\gamma \gamma^{\prime}}\right)\right]
\end{aligned}
$$

Using the definition of Laplace transform, $\mathbb{E}_{Z}[\exp (-Z s)]=\mathcal{L}_{Z}(s)$, and further simplification, we get

$$
\mathbb{P}\left\{\Gamma>\gamma, \Gamma^{\prime}>\gamma^{\prime} \mid R=r, R^{\prime}=r^{\prime}\right\}=\frac{\left(1-\gamma \gamma^{\prime}\right) \mathcal{L}_{Z}\left(\frac{1}{1-\gamma \gamma^{\prime}}\left(\frac{\gamma\left(1+\gamma^{\prime}\right) r^{\delta}}{P}+\frac{\gamma^{\prime}(1+\gamma)\left(r^{\prime}\right)^{\delta}}{P^{\prime}}\right)\right)}{\left[1+\gamma \frac{P^{\prime}}{P}\left(\frac{r}{r^{\prime}}\right)^{\delta}\right]\left[1+\gamma^{\prime} \frac{P}{P^{\prime}}\left(\frac{r^{\prime}}{r}\right)^{\delta}\right]}
$$




\section{Appendix 2: Derivation of JPDF Expression}

Assuming $\delta=4$, the JCCDF expression in (A1.10) can be rewritten as,

$$
\mathbb{P}\left\{\Gamma>\gamma, \Gamma^{\prime}>\gamma^{\prime} \mid R=r, R^{\prime}=r^{\prime}\right\}=M_{1} M_{2}
$$

where,

$$
\begin{aligned}
M_{1} & =\frac{1-\gamma \gamma^{\prime}}{\left[1+\gamma \frac{P^{\prime}}{P}\left(\frac{r}{r^{\prime}}\right)^{4}\right]\left[1+\gamma^{\prime} \frac{P}{P^{\prime}}\left(\frac{r^{\prime}}{r}\right)^{4}\right]}, \\
M_{2} & =\mathcal{L}_{Z}\left(\frac{1}{1-\gamma \gamma^{\prime}}\left(\frac{\gamma\left(1+\gamma^{\prime}\right) r^{4}}{P}+\frac{\gamma^{\prime}(1+\gamma)\left(r^{\prime}\right)^{4}}{P^{\prime}}\right)\right) .
\end{aligned}
$$

After some tedious but straight forward algebraic steps, it can be shown that

$$
\begin{aligned}
& M_{1}=\frac{1}{1+\gamma\left(\frac{\tilde{a}}{1-\tilde{a}}\right)}+\frac{1}{1+\gamma^{\prime}\left(\frac{1-\tilde{a}}{\tilde{a}}\right)}-1, \\
& M_{2}=\exp \{g(\sqrt{\tilde{a}}, \beta \tilde{\mu})+g(\sqrt{\tilde{a} / \alpha},(1-\beta) \tilde{\mu} \sqrt{\alpha})+g(\sqrt{1-\tilde{a}}, 1-\tilde{\mu})\},
\end{aligned}
$$

where, $\tilde{a}=\frac{1}{1+\frac{P}{P^{\prime}}\left(\frac{r^{\prime}}{r}\right)^{4}}, \tilde{\mu}=\frac{1}{1+\frac{\lambda^{\prime}}{\lambda} \sqrt{\frac{P^{\prime}}{P}}}$. The function $g$ in (A2.5) is defined as

$$
g(b, \nu)=-\nu c B\left(\frac{\pi}{2}-\tan ^{-1} \frac{b}{c}\right)
$$

where,

$$
B=\frac{\pi r^{2}}{\sqrt{P \tilde{a}}}\left(\lambda \sqrt{P}+\lambda^{\prime} \sqrt{P^{\prime}}\right) \quad \text { and } \quad c=\sqrt{\frac{\gamma\left(1+\gamma^{\prime}\right) \tilde{a}+\gamma^{\prime}(1+\gamma)(1-\tilde{a})}{1-\gamma \gamma^{\prime}}} .
$$

We can derive the JPDF by differentiating the JCCDF (A2.1) with respect to $\gamma$ and $\gamma^{\prime}$,

$$
f_{\Gamma, \Gamma^{\prime} \mid R, R^{\prime}}\left(\gamma, \gamma^{\prime} \mid r, r^{\prime}\right)=\frac{\partial^{2}}{\partial \gamma \partial \gamma^{\prime}} M_{1} M_{2}
$$


where $M_{1}$ and $M_{2}$ are given by (A2.4) and (A2.5), respectively. By solving (A2.8) it can be shown that the conditional JPDF

$$
\begin{aligned}
f_{\Gamma, \Gamma^{\prime} \mid R, R^{\prime}}\left(\gamma, \gamma^{\prime} \mid r, r^{\prime}\right)=M_{2} h\left(\frac{\partial M_{1}}{\partial \gamma} \frac{\partial c}{\partial \gamma^{\prime}}\right. & \left.+\frac{\partial M_{1}}{\partial \gamma^{\prime}} \frac{\partial c}{\partial \gamma}+\frac{\partial^{2} c}{\partial \gamma \partial \gamma^{\prime}} M_{1}\right) \\
& +M_{1} M_{2} \frac{\partial c}{\partial \gamma} \frac{\partial c}{\partial \gamma^{\prime}}\left(h^{2}+\frac{\partial h}{\partial c}\right)
\end{aligned}
$$

where,

$$
\begin{aligned}
h & =\frac{\ln M_{2}}{c}-B c\left[\frac{\beta \tilde{\mu} \sqrt{\tilde{a}}}{c^{2}+\tilde{a}}+\frac{(1-\beta) \tilde{\mu} \alpha \sqrt{\tilde{a}}}{c^{2} \alpha+\tilde{a}}+\frac{(1-\tilde{\mu}) \sqrt{1-\tilde{a}}}{c^{2}+1-\tilde{a}}\right], \\
\frac{\partial M_{1}}{\partial \gamma} & =-\frac{\tilde{a}(1-\tilde{a})}{(1+\tilde{a} \gamma-\tilde{a})^{2}}, \\
\frac{\partial M_{1}}{\partial \gamma^{\prime}} & =-\frac{\tilde{a}(1-\tilde{a})}{\left[\gamma^{\prime}(1-\tilde{a})+\tilde{a}\right]^{2}}, \\
\frac{\partial c}{\partial \gamma} & =\frac{1}{2 \gamma\left(1-\gamma \gamma^{\prime}\right)}\left(c-\frac{\gamma^{\prime}(1-\tilde{a})}{c}\right), \\
\frac{\partial c}{\partial \gamma^{\prime}} & =\frac{1}{2 \gamma^{\prime}\left(1-\gamma \gamma^{\prime}\right)}\left(c-\frac{\gamma \tilde{a}}{c}\right), \\
\frac{\partial^{2} c}{\partial \gamma \partial \gamma^{\prime}} & =\frac{1}{4\left(1-\gamma \gamma^{\prime}\right)^{2}}\left[3 c+\frac{1}{c}-\frac{\tilde{a}(1-\tilde{a})}{c^{3}}\right], \\
\frac{\partial h}{\partial c} & =-2 B\left[\frac{\beta \tilde{\mu} \tilde{a}^{3 / 2}}{\left(c^{2}+\tilde{a}\right)^{2}}+\frac{(1-\beta) \tilde{\mu} \tilde{a}^{3 / 2} \alpha}{\left(c^{2} \alpha+\tilde{a}\right)^{2}}+\frac{(1-\tilde{\mu})(1-\tilde{a})^{3 / 2}}{\left(c^{2}+1-\tilde{a}\right)^{2}}\right] .
\end{aligned}
$$




\section{Appendix 3: Approximating the $\alpha$ and $\beta$ Parameters of Gamma Distribution}

In this appendix, we derive the expressions for $\alpha$ and $\beta$ parameters through a heuristic approach to minimize the MSE between the approximation $f_{H}^{\mathrm{g}}(h)$ and the $\operatorname{PMF} f_{H}(h)$ of the handover count. First, we briefly describe a property of PPP with respect to the scaling of simulation space which will be used for the derivation of $\alpha$ and $\beta$ parameters.
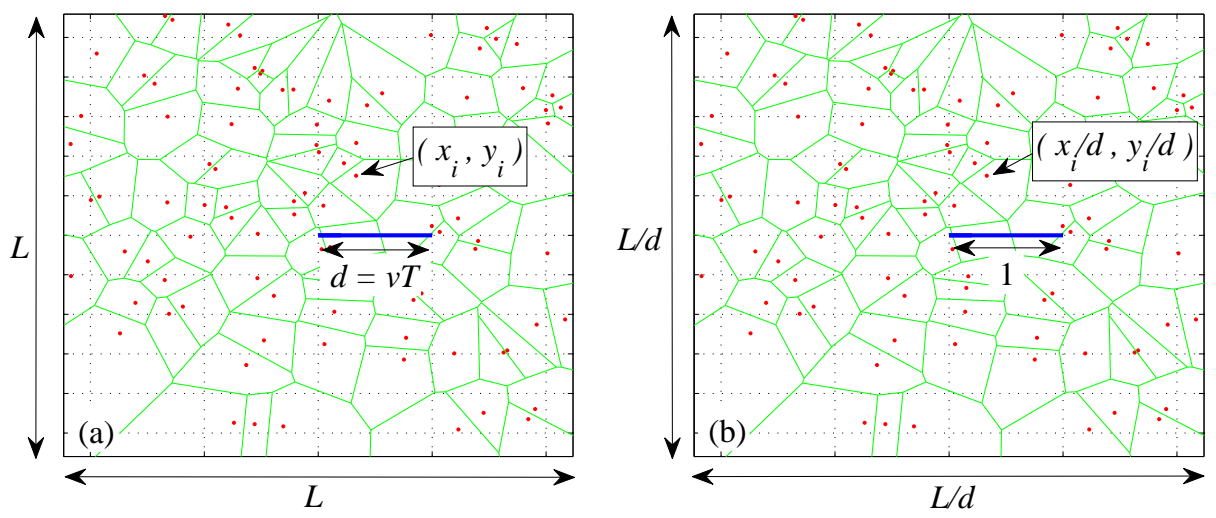

Figure A3.1: Illustration for scaling down the simulation space.

Consider the square shaped simulation space shown in Fig. A3.1(a) with an area $L^{2}$. The SBS locations are shown in red dots, and the UE trajectory is shown as blue line of length $d=v T$. The intensity of SBSs can be expressed as $\lambda=N_{\text {avg }} / L^{2}$, where $N_{\text {avg }}$ is the mean number of SBSs in the simulation area. Assume that the simulation space is scaled down by the UE travel distance $d$, i.e., all the coordinates are scaled down by $d$, for example, coordinates of a SBS location $\left(x_{i}, y_{i}\right)$ will be scaled down to $\left(\frac{x_{i}}{d}, \frac{y_{i}}{d}\right)$ as shown in Fig. A3.1(b). Therefore, the simulation area will also be scaled down to $(L / d)^{2}$. However, the mean number of SBSs $N_{\text {avg }}$ remains the same, and hence the intensity of SBSs can be expressed as $\lambda^{\prime}=N_{\mathrm{avg}} d^{2} / L^{2}=\lambda d^{2}$. Here, the term $\lambda^{\prime}$ is a function of $\lambda$ and $d$, therefore, the statistics of the handover count $H$ can be expressed in terms of $\lambda^{\prime}$ alone. For example, the mean number of handovers in (7.1) can be expressed as $E[H]=4 \sqrt{\lambda^{\prime}} / \pi$. Similarly, the parameters $\alpha$ and $\beta$ can also be approximated in terms of $\lambda^{\prime}$.

We obtained histogram of the handover count $H$ through extensive simulations in Matlab with a range of values for the SBS density $\lambda=\{100,200,300, \ldots, 10000\}$ SBSs $/ \mathrm{km}^{2}$ and the 
distance $d=\{0.033,0.1,0.2,0.4\} \mathrm{km}$. For each combination of $\lambda$ and $d$, we obtained one million samples of $H$ for constructing the $\mathrm{PMF} f_{H}(h)$. Then, we used the curve fitting tool in Matlab to obtain the $\alpha$ and $\beta$ parameters that provides a best fit of $f_{H}^{\mathrm{g}}(h)$ in $(7.5)$ to the PMF $f_{H}(h)$. By studying the characteristics of $\alpha$ and $\beta$ parameters with respect to the variations in $\lambda$ and $d$, we formulated

$$
\begin{aligned}
& \alpha=2.7+4 \sqrt{\lambda^{\prime}}=2.7+4 d \sqrt{\lambda}, \\
& \beta=\pi+\frac{0.8}{0.38+\sqrt{\lambda^{\prime}}}=\pi+\frac{0.8}{0.38+d \sqrt{\lambda}} .
\end{aligned}
$$

The accuracy of these approximations are justified in Fig. A3.2 where the theoretical expressions (A3.1) and (A3.2) are shown to tightly overlap with the plots obtained through simulations.
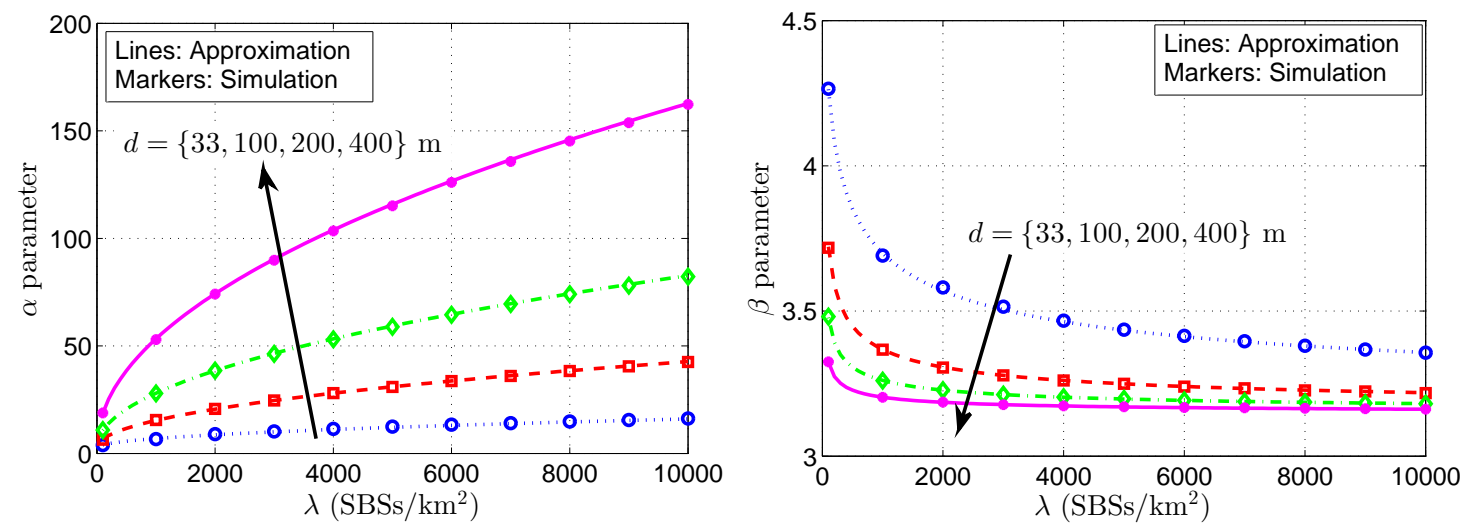

Figure A3.2: Approximations of the $\alpha$ and $\beta$ parameters. 


\section{Appendix 4: Approximating the $\mu$ and $\sigma^{2}$ Parameters of Gaussian Distribution}

In this appendix, we derive the expressions for $\mu$ and $\sigma^{2}$ in (7.9) to minimize the MSE between the approximation $f_{H}^{\mathrm{n}}(h)$ and the PMF $f_{H}(h)$ of the handover count. We equate $\mu$ to the mean of handover count expressed in (7.1) as

$$
\mu=\frac{4 v T \sqrt{\lambda}}{\pi}=\frac{4 d \sqrt{\lambda}}{\pi}
$$

Next, we obtain an expression for $\sigma^{2}$ using a method similar to the one explained in Appendix 3. We constructed the PMF $f_{H}(h)$ through simulations, and then used curve fitting tool in Matlab to obtain the $\sigma^{2}$ parameter that provides a best fitting of $f_{H}^{\mathrm{n}}(h)$ in $(7.9)$ to the PMF $f_{H}(h)$. By studying the characteristics of $\sigma^{2}$ parameter with respect to the variations in $\lambda$ and $d$, we formulated

$$
\sigma^{2}=0.07+0.41 \sqrt{\lambda^{\prime}}=0.07+0.41 d \sqrt{\lambda} .
$$

The accuracy of this approximation is justified in Fig. A4.1 where the expression (A4.2) is plotted in comparison with the plots obtained through simulations.

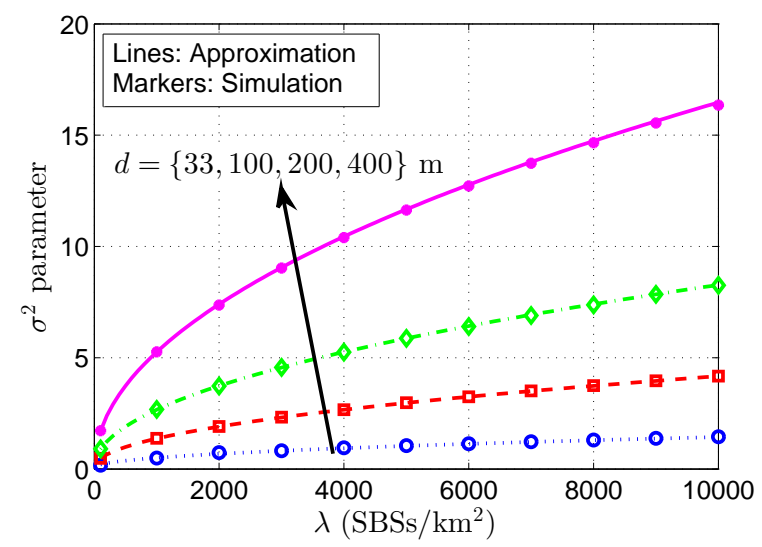

Figure A4.1: Approximation of the $\sigma^{2}$ parameter. 


\section{Appendix 5: Derivation of CRLB with Gamma Approximation for Handover Count PMF}

In this appendix, CRLB for velocity estimation will be derived by considering the handover count PMF in (7.5) that was derived using the gamma distribution. Taking the logarithm of PMF will provide the log-likelihood function, which will then be differentiated with respect to $v$. Finally, the derivative of log-likelihood function will be used to obtain the CRLB expression. Consider the handover count PMF,

$$
f_{H}^{\mathrm{g}}(h ; v)=\frac{\Gamma(\alpha, \beta h, \beta(h+1))}{\Gamma(\alpha)}
$$

where, the $\alpha$ and $\beta$ parameters are given by (7.6) and (7.7), respectively. By taking the logarithm of (A5.1) and differentiating with respect to $v$, we get

$$
\frac{\partial}{\partial v} \log f_{H}^{\mathrm{g}}(h ; v)=\frac{\partial}{\partial v} \log \Gamma(\alpha, \beta h, \beta(h+1))-\frac{\partial}{\partial v} \log \Gamma(\alpha)
$$

Consider the first term in the RHS of (A5.2). Let, $z_{1}=\beta h$ and $z_{2}=\beta(h+1)$. Then, we have

$$
\begin{aligned}
& \frac{\partial}{\partial v} \log \Gamma(\alpha, \beta h, \beta(h+1))=\frac{1}{\Gamma\left(\alpha, z_{1}, z_{2}\right)} \frac{\partial}{\partial v} \Gamma\left(\alpha, z_{1}, z_{2}\right) \\
& \quad=\frac{1}{\Gamma\left(\alpha, z_{1}, z_{2}\right)}\left[\frac{\partial}{\partial \alpha} \Gamma\left(\alpha, z_{1}, z_{2}\right) \frac{\mathrm{d} \alpha}{\mathrm{d} v}+\frac{\partial}{\partial z_{1}} \Gamma\left(\alpha, z_{1}, z_{2}\right) \frac{\mathrm{d} z_{1}}{\mathrm{~d} v}+\frac{\partial}{\partial z_{2}} \Gamma\left(\alpha, z_{1}, z_{2}\right) \frac{\mathrm{d} z_{2}}{\mathrm{~d} v}\right] .
\end{aligned}
$$

Each of the differentials in (A5.3) can be derived to be

$$
\begin{aligned}
\frac{\partial}{\partial \alpha} \Gamma\left(\alpha, z_{1}, z_{2}\right)= & \frac{{ }_{2} F_{2}\left(\alpha, \alpha ; \alpha+1, \alpha+1 ;-z_{1}\right) z_{1}^{\alpha}}{\alpha^{2}}-\frac{{ }_{2} F_{2}\left(\alpha, \alpha ; \alpha+1, \alpha+1 ;-z_{2}\right) z_{2}^{\alpha}}{\alpha^{2}} \\
& -\gamma\left(\alpha, z_{1}\right) \log \left(z_{1}\right)+\gamma\left(\alpha, z_{2}\right) \log \left(z_{2}\right), \\
\frac{\partial}{\partial z_{1}} \Gamma\left(\alpha, z_{1}, z_{2}\right)= & -e^{-z_{1}} z_{1}^{\alpha-1}, \quad \frac{\partial}{\partial z_{2}} \Gamma\left(\alpha, z_{1}, z_{2}\right)=e^{-z_{2}} z_{2}^{\alpha-1}, \\
\frac{\mathrm{d} \alpha}{\mathrm{d} v}= & 4 T \sqrt{\lambda}, \quad \frac{\mathrm{d} z_{1}}{\mathrm{~d} v}=\frac{-0.8 h T \sqrt{\lambda}}{(0.38+v T \sqrt{\lambda})^{2}}, \quad \frac{\mathrm{d} z_{2}}{\mathrm{~d} v}=\frac{-0.8(h+1) T \sqrt{\lambda}}{(0.38+v T \sqrt{\lambda})^{2}},
\end{aligned}
$$


where, $\gamma(\alpha, x)=\int_{0}^{x} t^{\alpha-1} e^{-t} \mathrm{~d} t$ is the lower incomplete gamma function, and ${ }_{2} F_{2}\left(a_{1}, a_{2} ; b_{1}, b_{2} ; z\right)$ is generalized hypergeometric function which can be expressed as

$$
{ }_{2} F_{2}\left(a_{1}, a_{2} ; b_{1}, b_{2} ; z\right)=\sum_{k=0}^{\infty} \frac{\left(a_{1}\right)_{k}\left(a_{2}\right)_{k}}{\left(b_{1}\right)_{k}\left(b_{2}\right)_{k}} \frac{z^{k}}{k !},
$$

where, $(a)_{0}=1$ and $(a)_{k}=a(a+1)(a+2) \ldots(a+k-1)$, for $k \geq 1$.

Now, consider the second term in RHS of (A5.2), $\frac{\partial}{\partial v} \log \Gamma(\alpha)=\frac{\partial \log \Gamma(\alpha)}{\partial \alpha} \frac{\partial \alpha}{\partial v}$. It is well known that the logarithmic derivative of the gamma function is the digamma function $\psi(\cdot)$. Therefore,

$$
\frac{\partial}{\partial v} \log \Gamma(\alpha)=\psi(\alpha) \frac{\partial \alpha}{\partial v}=\psi(\alpha) \frac{\partial}{\partial v}(2.7+4 v T \sqrt{\lambda})=4 T \sqrt{\lambda} \psi(\alpha)
$$

Using the equations (A5.3)-(A5.5), we can express (A5.2) as:

$$
\begin{aligned}
\frac{\partial}{\partial v} \log f_{H}^{\mathrm{g}}(h ; v)= & \frac{4 T \sqrt{\lambda} \beta^{\alpha}}{\alpha^{2} \Gamma(\alpha, \beta h, \beta(h+1))}\left[h^{\alpha}{ }_{2} F_{2}(\alpha, \alpha ; \alpha+1, \alpha+1 ;-\beta h)\right. \\
& \left.-(h+1)^{\alpha}{ }_{2} F_{2}(\alpha, \alpha ; \alpha+1, \alpha+1 ;-\beta(h+1))\right] \\
& -\frac{4 T \sqrt{\lambda}}{\Gamma(\alpha, \beta h, \beta(h+1))}[\gamma(\alpha, \beta h) \log (\beta h)-\gamma(\alpha, \beta(h+1)) \log (\beta(h+1))] \\
& +\frac{0.8 T \sqrt{\lambda} \beta^{\alpha-1} e^{-\beta h}\left[h^{\alpha}-e^{-\beta}(h+1)^{\alpha}\right]}{\Gamma(\alpha, \beta h, \beta(h+1))(0.38+v T \sqrt{\lambda})^{2}}-4 T \sqrt{\lambda} \psi(\alpha) .
\end{aligned}
$$

By squaring (A5.6) and evaluating the expectation over $H$, we obtain the Fisher information as

$$
I(v)=\mathbb{E}\left[\left(\frac{\partial \log f_{H}^{\mathrm{g}}(H ; v)}{\partial v}\right)^{2}\right]
$$

By taking the reciprocal of Fisher information, we can express the CRLB for $\hat{v}$ as in (7.12). 


\section{Appendix 6: Regularity Condition}

By taking natural logarithm of the JPDF in (8.3) and then differentiating with respect to $v$, we get

$$
\frac{\partial \log f_{\boldsymbol{T}}(\boldsymbol{t} ; v)}{\partial v}=\frac{N}{v}+\sum_{n=0}^{N-1} \frac{t_{n}}{f_{L}\left(l_{n}\right)} \frac{\partial f_{L}\left(l_{n}\right)}{\partial l_{n}} .
$$

The differential term in (A6.1) can be derived to be

$$
\frac{\partial f_{L}\left(l_{n}\right)}{\partial l_{n}}=\frac{2}{l_{n}}\left(f_{L}\left(l_{n}\right)+f_{L}^{\prime}\left(l_{n}\right)\right),
$$

where,

$$
\begin{aligned}
& f_{L}^{\prime}(l)=\int_{0}^{\pi} \int_{0}^{\pi-\alpha}\left(\frac{2 \pi^{3} \lambda^{2} l^{4} \rho_{\alpha}^{3} \rho_{\beta} b_{0}^{2}\left(1-\pi \lambda l^{2} V_{2}(\alpha, \beta)\right)}{\sin (\alpha+\beta)}\right. \\
&\left.+\frac{\pi^{3} \lambda^{2} l^{4} \rho_{\alpha} \rho_{\beta} c_{0} V_{2}(\alpha, \beta)}{\sin (\alpha+\beta)}\right) e^{-\pi \lambda l^{2} V_{2}(\alpha, \beta)} \mathrm{d} \beta \mathrm{d} \alpha .
\end{aligned}
$$

By substituting (A6.2) into (A6.1), we get,

$$
\frac{\partial \log f_{\boldsymbol{T}}(\boldsymbol{t} ; v)}{\partial v}=\frac{1}{v}\left(3 N+2 \sum_{n=0}^{N-1} \frac{f_{L}^{\prime}\left(l_{n}\right)}{f_{L}\left(l_{n}\right)}\right) .
$$

Given $N$, to check whether the JPDF satisfies the regularity condition, we take the expectation of (A6.3) w.r.t. $L$ as,

$$
\begin{aligned}
E\left[\frac{\partial \log f_{\boldsymbol{T}}(\boldsymbol{t} ; v)}{\partial v}\right] & =\frac{1}{v} E_{L}\left(3 N+2 \sum_{n=0}^{N-1} \frac{f_{L}^{\prime}\left(l_{n}\right)}{f_{L}\left(l_{n}\right)}\right) \\
& =\frac{1}{v}\left(3 N+2 \sum_{n=0}^{N-1} E_{L}\left[\frac{f_{L}^{\prime}\left(l_{n}\right)}{f_{L}\left(l_{n}\right)}\right]\right) .
\end{aligned}
$$

The expectation function w.r.t. $L$ in (A6.4) can be derived as

$$
E_{L}\left[\frac{f_{L}^{\prime}\left(l_{n}\right)}{f_{L}\left(l_{n}\right)}\right]=\int_{0}^{\infty} f_{L}^{\prime}(l) \mathrm{d} l=-1.5 .
$$


The last step in (A6.5) was obtained by numerically evaluating the integral $\int_{0}^{\infty} f_{L}^{\prime}(l) \mathrm{d} l$ in MATLAB with an error tolerance of $10^{-6}$. By substituting (A6.5) into (A6.4), we get,

$$
E\left[\frac{\partial \log f_{\boldsymbol{T}}(\boldsymbol{t} ; v)}{\partial v}\right]=\frac{3 N+2(-1.5) N}{v}=0
$$

Since the expectation function in (A6.6) equates to zero irrespective of the values of $N$ and $v$, the JPDF in (8.3) satisfies the regularity condition for all $N$ and $v$. 


\section{Appendix 7: CRLB Derivation}

By squaring (A6.3) and taking the expectation, we get,

$$
\begin{aligned}
& E\left[\left(\frac{\partial \log f_{\boldsymbol{T}}(\boldsymbol{t} ; v)}{\partial v}\right)^{2}\right]= \frac{1}{v^{2}} E[ \\
&\left.\hline N^{2}+4\left(\sum_{n=0}^{N-1} \frac{f_{L}^{\prime}\left(l_{n}\right)}{f_{L}\left(l_{n}\right)}\right)^{2}+12 N \sum_{n=0}^{N-1} \frac{f_{L}^{\prime}\left(l_{n}\right)}{f_{L}\left(l_{n}\right)}\right] \\
&=\frac{1}{v^{2}} E_{N}\left(9 N^{2}+4 \sum_{n=0}^{N-1} E_{L}\left[\left(\frac{f_{L}^{\prime}\left(l_{n}\right)}{f_{L}\left(l_{n}\right)}\right)^{2}\right]\right. \\
&\left.+8 \sum_{m<n} E_{L}\left[\frac{f_{L}^{\prime}\left(l_{n}\right) f_{L}^{\prime}\left(l_{m}\right)}{f_{L}\left(l_{n}\right) f_{L}\left(l_{m}\right)}\right]+12 N \sum_{n=0}^{N-1} E_{L}\left[\frac{f_{L}^{\prime}\left(l_{n}\right)}{f_{L}\left(l_{n}\right)}\right]\right) .
\end{aligned}
$$

The first two expectations w.r.t. $L$ in $(\mathrm{A} 7.1)$ can be derived as follows:

$$
E_{L}\left[\left(\frac{f_{L}^{\prime}\left(l_{n}\right)}{f_{L}\left(l_{n}\right)}\right)^{2}\right]=\int_{0}^{\infty}\left(\frac{f_{L}^{\prime}(l)}{f_{L}(l)}\right)^{2} f_{L}(l) \mathrm{d} l=3.345
$$

The last step in (A7.2) was obtained by numerically evaluating the integral in MATLAB with an error tolerance of $10^{-6}$. Next,

$$
\begin{aligned}
E_{L}\left[\frac{f_{L}^{\prime}\left(l_{n}\right) f_{L}^{\prime}\left(l_{m}\right)}{f_{L}\left(l_{n}\right) f_{L}\left(l_{m}\right)}\right] & =\iint_{0}^{\infty} \frac{f_{L}^{\prime}\left(l_{n}\right) f_{L}^{\prime}\left(l_{m}\right)}{f_{L}\left(l_{n}\right) f_{L}\left(l_{m}\right)} f_{L}\left(l_{n}\right) f_{L}\left(l_{m}\right) \mathrm{d} l_{n} \mathrm{~d} l_{m} \\
& =\int_{0}^{\infty} f_{L}^{\prime}\left(l_{m}\right)\left(\int_{0}^{\infty} f_{L}^{\prime}\left(l_{n}\right) \mathrm{d} l_{n}\right) \mathrm{d} l_{m}
\end{aligned}
$$

Substituting (A6.5) into the above equation, we get,

$$
E\left[\frac{f_{L}^{\prime}\left(l_{n}\right) f_{L}^{\prime}\left(l_{m}\right)}{f_{L}\left(l_{n}\right) f_{L}\left(l_{m}\right)}\right]=-1.5 \int_{0}^{\infty} f_{L}^{\prime}\left(l_{m}\right) \mathrm{d} l_{m}=2.25
$$

By substituting (A6.5), (A7.2) and (A7.3) into (A7.1), we get,

$$
E\left[\left(\frac{\partial \log f_{\boldsymbol{T}}(\boldsymbol{t} ; v)}{\partial v}\right)^{2}\right]=\frac{4.38}{v^{2}} E[N]
$$


The expectation of $N$ in (A7.4) is basically the mean number of handovers, the expression for which is derived in [97] as

$$
E[N]=4 v T_{\mathrm{w}} \sqrt{\lambda} / \pi
$$

By substituting (A7.5) into (A7.4), we get,

$$
E\left[\left(\frac{\partial \log f_{\boldsymbol{T}}(\boldsymbol{t} ; v)}{\partial v}\right)^{2}\right]=\frac{4.38}{v^{2}} \frac{4 v T_{\mathrm{w}} \sqrt{\lambda}}{\pi}=\frac{17.52 T_{\mathrm{w}} \sqrt{\lambda}}{\pi v}
$$

By substituting the above equation into (8.4), we obtain the CRLB as expressed in (7.12). This completes the proof. 
VITA

\section{ARVIND MERWADAY}

Born, Bengaluru, India

2008

B.E., Telecommunication Engineering

BMS College of Engineering

Bengaluru, India

2008-2011

Applications Engineer

Cypress Semiconductor Technology India Pvt. Ltd.

Bengaluru, India

$2012-2014$

Teaching Assistant

Department of Electrical and Computer Engineering

Florida International University

Miami, Florida

$2014-2016$

Doctoral Candidate

Florida International University

Miami, Florida

Research Assistant

MPACT Research Lab

Florida International University

Miami, Florida

2015

M.S., Electrical Engineering

Florida International University

Miami, Florida

Research Scientist Intern

Intel Corporation

Hillsboro, Oregon

\section{PUBLICATIONS AND PRESENTATIONS}

[1] O. Jonah, A. Merwaday, S. V. Georgakopoulos and M. M. Tentzeris, "Spiral resonators for optimally efficient strongly coupled magnetic resonant systems," Wireless Power Transfer, vol. 1, no. 1, pp. 21-26, Mar. 2014.

[2] A. Merwaday and I. Guvenc, "Handover count based velocity estimation and mobility state detection in dense HetNets," IEEE Trans. Wireless Commun. (TWC), Mar. 2016. 
[3] A. Merwaday, I. Guvenc, W. Saad, A. Mehbodniya, and F. Adachi, "Sojourn time based velocity estimation in small cell poisson networks," IEEE Commun. Lett., vol. 20, no. 2, pp. 340-343, Nov. 2015.

[4] A. Merwaday, S. Mukherjee, and I. Guvenc, "Capacity analysis of LTE-Advanced HetNets with reduced power subframes and range expansion," EURASIP J. Wireless Commun. Networking, vol. 2014, no. 189, Nov. 2014.

[5] A. Merwaday and I. Guvenc, "Optimization of energy efficiency and spectrum efficiency in HetNets," IET Electronics Lett., Apr. 2016.

[6] A. Merwaday, A. Tuncer, A. Kumbhar, and I. Guvenc, "Unmanned aerial base stations for public safety communications," submitted to IEEE Veh. Technol. Mag., Jan. 2016.

[7] A. Merwaday, M. Yuksel, T. Quint, I. Guvenc, W. Saad, and N. Kapucu, "Incentivizing spectrum sharing via subsidy regulations," submitted to IEEE Trans. Mobile Computing (TMC), Jan. 2016.

[8] A. Merwaday and I. Guvenc, "Handover count based UE velocity estimation in hyper-dense heterogeneous wireless networks," in Proc. IEEE Workshop on Heterogeneous and Small Cell Networks (HetsNets), Co-located with IEEE Global Telecommun. Conf. (GLOBECOM), San Diego, CA, Dec. 2015, pp. 1-6.

[9] A. Merwaday and I. Guvenc, "UAV assisted heterogeneous networks for public safety communications," in Proc. IEEE Workshop on D2D and Public Safety Commun. (WDPC), Co-located with IEEE Wireless Commun. and Networking Conf. (WCNC), New Orleans, LA, Mar. 2015, pp. 329-334.

[10] A. Merwaday, N. Rupasinghe, I. Guvenc, W. Saad, and M. Yuksel, "USRP-based indoor channel sounding for D2D and multi-hop communications," in Proc. IEEE Wireless and Microwave Technol. Conf. (WAMICON), Tampa, FL, June 2014, pp. 1-6.

[11] A. Merwaday, S. Mukherjee, and I. Guvenc, "Hetnet capacity with reduced power subframes," in Proc. IEEE Wireless Commun. and Networking Conf. (WCNC), Istanbul, Turkey, Apr. 2014, pp. 1380-1385.

[12] A. Merwaday, S. Mukherjee, and I. Guvenc, "On the capacity analysis of hetnets with range expansion and eICIC," in Proc. IEEE Global Telecommun. Conf. (GLOBECOM), Atlanta, GA, Dec. 2013, pp. 4257-4262.

[13] M. Simsek, A. Merwaday, N. Correal, and I. Guvenc, "Device-to-device discovery based on 3GPP system level simulations," in Proc. Int. Workshop on D2D Commun. With and Without Infrastructure, Co-located with IEEE Global Telecommun. Conf. (GLOBECOM), Atlanta, GA, Dec. 2013, pp. 555-560. 